\title{
Reference
}

\section{NBSIR 80-1976}

\section{Numerical Solution of Linear Difference Equations}

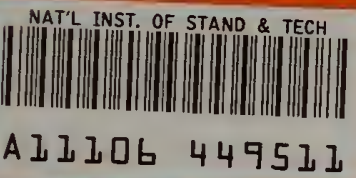

Caniel $W$ i-ozler

viathematical Analysis Division Center for Assiic: Mathernatics National Engıneering Laboratory Natioraa: Bureau of Standards i.S. Depariment uf Con:merce Washington, OC 2C234

March 1980

Final Repcrt

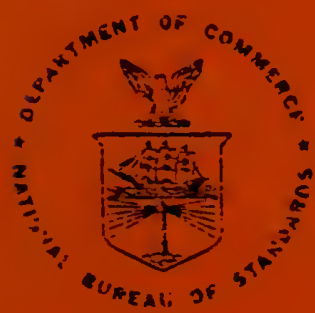

U.S. DEPAGTMENT DF COMMERCE

NATONAL BLREAL OF STANDARDS

$-Q C$

100

.456

$80-1976$

1980 

$\because$

\section{NUMERICAL SOLUTION OF LINEAR}

DIFFERENCE EQUATIONS

Daniel W. Lozier

Mathematical Analysis Division

Center for Applied Mathematics National Engineering Laboratory

National Bureau of Standards

U.S. Department of Commerce

Washington, D.C. 20234

March 1980

Final Report

U.S. DEPARTMENT OF COMMERCE, Philip M. Klutznick, Secretary

Luther H. Hodges, Jr., Deputy Secretary

Jordan J. Baruch, Assistant Secretary for Science and Technology

NATIONAL BUREAU OF STANDARDS, Ernest Ambler, Director 


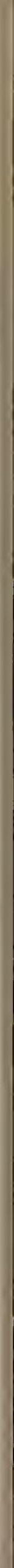


ABS TRACT

Consider a given homogeneous or inhomogeneous linear difference equation $\quad \sum_{s=0}^{\ell} d_{s}(r) y(r+s)=g(r)$ where $\ell \geq 2$ and $r=0,1,2, \ldots$. Suppose $y$ is a solution of this equation and $u, v$ are solutions of the homogeneous form of this equation such that $u(r) / v(r) \rightarrow 0$, $\mathrm{y}(r) / \mathrm{v}(r) \rightarrow 0, \mathrm{u}(\mathrm{r}) / \mathrm{y}(\mathrm{r}) \rightarrow 0$. It is known that under these circumstances algorithms for the computation of $y$ based on forward recurrence or backward recurrence, such as the Miller algorithm, are numerically unstable.

Stable algorithms, such as the method of olver in the case $\ell=2$, have been based on approximating $y(r)$ by the solutions $y_{n}(r)$ of a certain sequence of boundary value problems. More specifically, $\mathrm{y}_{\mathrm{n}}(r)$ is a solution of the difference equation that coincides with $y(r)$, over some fixed initial range of $r$, say $r=i, i+1, \ldots, i+j-1$, and satisfies $y_{n}(r)=0$ for $r=n, n+1, \ldots, n+l-j-1$. Here $j$ is an integer whose value depends on the asymptotic behavior of the chosen solution $y(r)$ and $n$ is an arbitrary large integer. Boundary value problems of this type are shown to be equivalent to two initial value problems of order $j$ and $l-j$ by factorization of the linear difference operator. The solution of the problem of order $j$ is obtained by forward recurrence; the solution of the other problem is obtained by backward recurrence.

The algorithm is specified completely for a broad class of linear difference operators. This class includes, for example, every constantcoefficient operator. Convergence of $y_{n}(r)$ to $y(r)$ as $n \rightarrow \infty$ for fixed $r$ is proved and an expansion of the truncation error is derived. Numerical stability is demonstrated under appropriate conditions. The method is tested by numerical examples involving fourth-order equations with variable coefficients. 
PREFACE

This paper was submitted as a Ph.D. thesis in the Interdisciplinary Applied Mathematics Program of the University of Maryland at College Park. The Ph.D. degree was awarded on December 18, 1979.

\section{ACKNOWLEDGMENTS}

I wish to acknowledge the aid and encouragement rendered by my thesis professor, Dr. Frank W.J. Olver. His leadership was superb. I also wish to express my appreciation to Dr. Daniel Shanks for directing me to the literature on almost periodic functions, to Donald J. Orser for helping with MACSYMA, to Richard N. Freemire for producing the figures, to Katherine Mesztenyi for typing the manuscript, and to my wife Elaine for her patience.

Computer support was provided by the Center for Applied Mathematics, National Bureau of Standards, and the Mathlab Group, Laboratory for Computer Science, Massachusetts Institute of Technology . 


\section{CONTENTS}

\section{INTRODUCTION}

1.1. Miller's and Olver's Algorithms . . . . . . . . . . . 1

1.2. Factorization of Linear Difference Equations . . . . . . 7

1.3. A Preliminary Development of the Algorithm . . . . . . 22

2. CLASSIFICATION OF SEQUENCES

2.1. Constant-Coefficient Operators . . . . . . . . . . . 36

2.2. Separable Operators ................ . . 42

2.3. The General Classification . . . . . . . . . . . 51

3. THE GENERAL ALGORITHM

3.0. Preliminaries and Overview . . . . . . . . . . 55

3.1. Existence and Uniqueness . . . . . . . . . . . . 58

3.2. Convergence . . . . . . . . . . . . . . 63

3.3. Stability . . . . . . . . . . . . . . 70

4. NUMERICAL EXAMPLES

4.0. Introduction ................. . . 74

4.1. Examples Involving the Homogeneous JYIK Equation. . . . 85

4.2. Examples Involving the Inhomogeneous JYIK Equation . . . 117

4.3. Examples Involving the Homogeneous JYPQ Equation . . . . 121

4.4. Examples Involving the Inhomogeneous JYPQ Equation . . . 139

5. SUMMARY AND CONCLUSIONS . . . . . . . . . . . . . . . . . . 144

APPENDIX . . . . . . . . . . . . . . . . . . 154

REFERENCES . . . . . . . . . . . . . . . . . . 158 
1. Numerical coefficients, JYIK operator, $x_{1}=1, x_{2}=10 . . . . .990$

2. $(0,1)$-factorization, JYIK operator, $x_{1}=1, x_{2}=10$. . . . . . 93

3. $(0,2)$-iactorization, JYIK operator, $x_{1}=1, x_{2}=10$. . . . . 96

4. $(0,3)$-factorization, JYIK operator, $\mathrm{x}_{1}=1, \mathrm{x}_{2}=10 \ldots . . . .99$

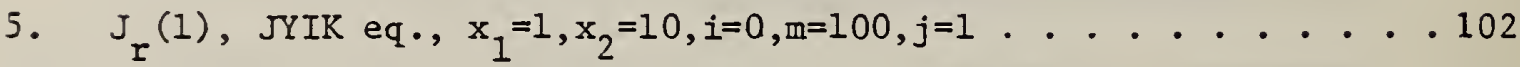

6. $I_{I}(10), J Y I K$ eq., $x_{1}=1, x_{2}=10, i=0, m=100, j=2 \ldots . . . . . . .103$

7. $(-)^{r_{K}} K_{r}(10)$, JYIK eq., $x_{1}=1, x_{2}=10, i=0, m=100, j=3 . . . . . .104$

8. $Y_{r}(1)$, JYIK eq., $x_{1}=1, x_{2}=10, i=0, m=100, j=4 \ldots . . . . . . .105$

9. $I_{r}(1), \operatorname{JYIK}$ eq., $x_{1}=10, x_{2}=1, i=0, m=100, j=1 . . . . . . . .107$

10. $J_{I}(10), J Y I K$ eq. $x_{1}=10, x_{2}=1, i=0, m=100, j=2 \ldots . . . . . .108$

11. $Y_{I}(10)$, JYIK eq., $x_{1}=10, x_{2}=1, i=0, m=100, j=3 . . . . . . .109$

12. $(-)^{I_{K}} K_{\Gamma}(1)$, JYIK eq., $x_{1}=10, x_{2}=1, i=0, m=100, j=4 \ldots . . . . .110$

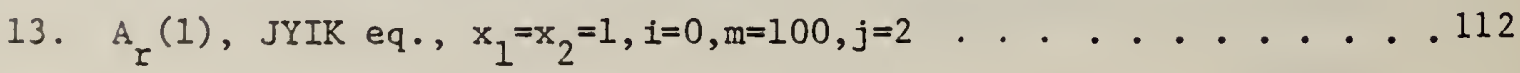

14. $J_{I}(1), \operatorname{JYIK}$ eq., $x_{1}=\gamma_{2}=1, i=0, m=100, j=2 \ldots . . . . . . .113$

15. $I_{I}(1), \operatorname{JIK}$ eq., $x_{1}=x_{2}=1, i=0, m=100, j=2 \ldots . . . . . . .114$ 


\section{TABLES (Continued)}

16. $Y_{r}(1)$, JYIK eq., $x_{1}=x_{2}=1, i=0, m=100, j=4$. . . . . . . . .

17. $(-)^{\Gamma_{K}}(1)$, JYIK eq., $x_{1}=x_{2}=1, i=0, m=100, j=4 \ldots . . . . . .116$

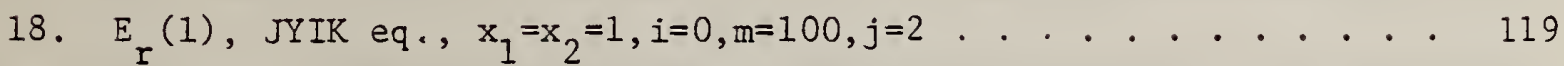

19. $H_{r}(0.1)$, JYIK eq., $x_{1}=x_{2}=0.1, i=0, m=50, j=2 \ldots \ldots 120$

20. Numerical coefficients, JYPQ operator, $\mu=0, x_{1}=1, x_{2}=0.5 . .$. .

21. (0.1)-factorization, JYPQ operator, $\mu=0, x_{1}=1, x_{2}=0.5 \ldots . .$.

22. $(0,3)$-factorization, JYPQ operator, $\mu=0, x_{1}=1, x_{2}=0.5 \cdots \cdot \cdot$

23. $J_{r}(1), J Y P Q$ eq., $\mu=0, x_{1}=1, x_{2}=0.5, i=0, m=100, j=1$. . . . .

24. $P_{r}(0.5), J Y P Q$ eq., $\mu=0, x_{1}=1, x_{2}=0.5, i=0, m=100, j=3 \ldots . . . \cdot$

25. $Q_{r}(0.5)$, JYPQ eq., $\mu=0, x_{1}=1, x_{2}=0.5, i=0, m=100, j=3 \ldots . . .$.

26. $Y_{r}(1), J Y P Q$ eq., $\mu=0, x_{1}=1, x_{2}=0.5, i=0, m=100, j=4 \ldots . . . \cdots$

27. $P_{r}^{1}(0.5)$, JYPQ eq., $\mu=1, x_{1}=1, x_{2}=0.5, i=0, m=100, j=3 \ldots . . . . \cdot$

28. $Q_{Y}^{1}(0.5), J Y P Q$ eq., $\mu=1, x_{1}=1, x_{2}=0.5, i=0, m=100, j=3 \ldots . . . . \cdot$

29. $E_{r}(1), J Y P Q$ eq., $\mu=1, x_{1}=1, x_{2}=0.5, i=0, m=100, j=3 . \ldots . . .$.

30. $\mathrm{H}_{\mathrm{r}}(0.1)$, JYPQ eq., $\mu=1, \mathrm{x}_{1}=1, \mathrm{x}_{2}=0.5, i=0, \mathrm{~m}=100, j=1$ 


\section{FIGURES}

1. The operator $\mathrm{D}^{\mathrm{i}}$. . . . . . . . . . . . . 9

2. The matrix $D_{j}^{i}$. . . . . . . . . . . . . . 9

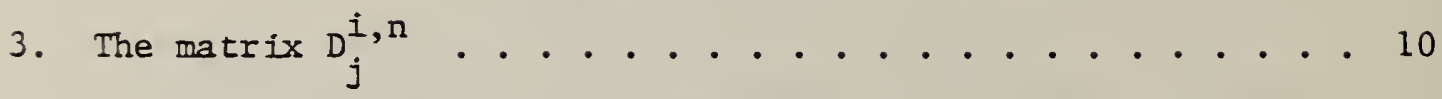

4. The factorization (1.2.5) ............. 16

5. The roots of $(2.1 .2)$. . . . . . . . . . 39

6. Fragment of FORTRAN subroutine, JYIK operator . . . . . . 76

7. Fragment of FORTRAN subroutine, JYPQ operator . . . . . 78 


\section{CHAPTER 1. INTRODUCTION}

\subsection{Miller's and Olver's Algorithms}

Linear recurrence relations (linear difference equations) are satisfied by many of the higher transcendental functions of mathematics, and they arise quite naturally in applications of mathematics. Discretization of ordinary differential equations in numerical analysis is one important area in which they occur. Because of their special form, linear recurrence relations are especially well suited to the construction of compuring algorithms. Stability requirements in the case of second-order homogeneous relations led to the well-known Miller backward recurrence algorithm [ 5 ], which was developed for the computation of modified Bessel functions. This reference was published in 1952. The review paper of Gautschi [9] provides a survey of algorithms and applications in the case of second-order homogeneous equations. Nowadays, the name of Miller often attends when a minimal solution (defined below) of a homogeneous equation of arbitrary order is computed using backward recurrence [28]. These algorichms always involve backward recurrence from assumed starting values (since these are not known in general) followed by a normalization procedure, such as matching one value of the computed solution with the desired solution in order to arrive at the proper scale factor which relates these two solutions.

A different class of algorithms becomes necessary when "intermediate" solutions of a linear recurrence relation are to be computed. Intuitively speaking, an intermediate solution is one for which two other solutions can be found such that one dominates, and the other is dominated by, the intermediate solution. We are speaking here of dominance in the following sense: 
if $a=(a(0), a(1), \ldots)$ and $b=(b(0), b(1), \ldots)$ are two infinite sequences ${ }^{\dagger}$ then $b$ dominates $a$ whenever $a(r) / b(r) \rightarrow 0$ as $r \rightarrow \infty$.

Neither forward recurrence nor the Miller algorithm is numerically stable for an intermediate solution. This is because recurrence in either direction, when carried out in finite precision, will be contaminated by small components of the other solutions. An analysis of the propagation of these rounding errors appears in [18]. In the forward direction, as the recurrence proceeds through more and more steps, the dominant solutions grow and eventually overwhelm the desired intermediate solution. Since our concept of dominance is really dominance "at infinity", the situation for recurrence in the backward direction is not completely analogous. However, if we start at a sufficiently large value of $r$ and recur backward, other solutions grow at a faster rate than the wanted intermediate solution as $r$ decreases.

In addition to intermediate solutions, a difference equation has minimal (or "recessive") and maximal (or "dominant") solutions. Intuitively, a minimal solution does not dominate, and a maximal solution is not dominated by, any other solution of the difference equation as $r \rightarrow \infty$. Ultimately, forward recurrence is stable for maximal solutions since the error cannot grow more rapidly than the solution. Similarly, backward recurrence is stable for minimal solutions, at least in a region of sufficiently large $r$.

\footnotetext{
Te shall regard the solutions of a linear difference equation, as well as the coefficlents of the equation, as complex-valued functions of a discrete real variable. We shall assume that this discrete real variable proceeds in unit increments; thus the solutions, as well as the coefficients, of linear difference equations are complex sequences. We shall be interested only in those linear difference equations whose coefficient sequences are of Infinite length.
} 
The lowest order of difference equation for which an intermediate solution can exist, and then only when the equation is inhomogeneous, is two. Such an equation is of the form

$(1.1 .1) \quad a(r) y(r-1)-b(r) y(r)+c(r) y(r+1)=d(r), r=1,2, \ldots$.

Since the general solution is

$$
y(r)=\alpha u(r)+\beta v(r)+h(r), r=0,1,2, \ldots,
$$

where $\alpha$ and $\beta$ are constants, $u=(u(0), u(1), \ldots)$ and $v=(v(0), v(1)$, ...) are linearly independent solutions of the homogeneous equation, and $h=(h(0), h(1), \ldots)$ is a particular solution of $(1.1 .1)$, there can be at most three asymptotically distinct types of behavior of solutions as $r \rightarrow \infty$. Let us assume that $v$ dominates $u$ and that $h$ is an intermediate solution. In addition, assume $u(0) \neq 0$ and $v(r) \neq 0$ for a11 surficiently large $r$. Under these assumptions a stable algorithm for computing intermediate solutions of (1.1.1) was presented by Olver in [21]. If $y=(y(0), y(1), \ldots)$ is the desired intermediate solution, OIver's algorithm approximates $y$ by a sequence of solutions $y_{n}=\left(y_{n}(0), y_{n}(1)\right.$, ...) such that $y_{n}(0)=y(0)$ and $y_{n}(n)=0$ where $n$ is an appropriately chosen positive integer. Note that only one initial value of the wanted intermediate solution is required to determine the sequence of approximations. The sequence is shown to converge pointwise to $y$ as $n \rightarrow \infty$. Olver's algorithm efficiently produces the first n-1 unknown terms of $y_{n}$ as the solution of a tridiagonal linear system of order $n-1$. The procedure used for solving these linear equations is equivalent to Gaussian elimination without pivoting. It consists, in effect, of a forward elimination stage followed by a back substitution stage. The algorithm is constructed so that the numerical stability of this procedure may be assessed. The 
forward elimination stage is realized by two forward recurrences. One of these uses the homogeneous form of (1.1.1) to generate a dominant solution $p=(p(0), p(1), \ldots)$. The initial values used for starting this recurrence are $p(0)=0$ and $P(1)=1$, and the solution $p$ is asymptotically proportional to $\mathrm{v}$. Therefore, the rounding errors ultimately propagate in proportion to $\mathrm{p}$, although they could propagate faster than $\mathrm{p}$ at first (if $p$ is essentially proportional to $u$ for early values of $r$, corresponding to an excessively small value of $u(0)$ ). A combined algorithm that employs both Miller's algorithm and Olver's algorithm in adjacent ranges of $r$ is proposed in [22].

The second forward recurrence in the forward elimination stage of Olver's algorithm uses the inhomogeneous first-order equation

$$
a(r) e(r-1)-c(r) e(r)=d(r) p(r), r=1,2, \ldots,
$$

where the solution $e=(e(0), e(1), \ldots)$ satisfies $e(0)=y(0)$. Here two distinct asymptotic forms of behavior may exist, but there is no reason to expect that the calculation of $e$ will be asymptotically unstable in general. However, as was the case for the solution $p$, there may be applications where rounding errors grow more rapidly then $e(r)$ over some finite initial range of values of $r$.

The back substitution consists of backward recurrence of the inhomogeneous first-order equation

$$
p(r+1) y_{n}(r)-p(r) y_{n}(r+1)=e(r), r=n-1, n-2, \ldots, 1 \text {, }
$$

starting with $y_{n}(n)=0$. The stability of this backward recurrence is guaranteed in the "asymptotic region" belonging to the chosen solution $y(r)$, that is, the infinite range of $r$ which is such that the asymptotic 
behavior of $y(r)$ relative to $u(r)$ and $v(r)$ is maintained.

Olver's method of solving the tridiagonal system leads to a very convenient representation of the truncation error. For each $r$ and for all $\mathrm{n} \geq \mathrm{r}$, the expansion

$$
y(r)=y_{n}(r)+p(r) \sum_{s=n}^{\infty} \frac{e(s)}{p(s) p(s+1)}
$$

is valid. Consequently, for each $r$ and $n \geq r$ and $v \geq 1$ we have

$$
y_{n+v}(r)-y_{n}(r)=p(r) \sum_{s=n}^{n+v-1} \frac{e(s)}{p(s) p(s+1)}
$$

This expansion is useful as a basis for deciding on an appropriate value of n, i.e., for deciding when to stop the forward elimination and begin the back substitution. Suppose that for particular values of $\varepsilon>0$ and $r$, say $r=m$, it is desired that

$$
\left|y(m)-y_{n}(m)\right|<\varepsilon
$$

A value of $v(\geq 1)$ is selected and $y(m)$ is approximated by $y_{n+,}(m)$. The problem then is to determine the smallest value of $n$ such that

$$
\left|y_{n+v}(m)-y_{n}(m)\right|<\varepsilon
$$

This is done by continuing the forward elimination until $r=m$ at which point calculation of the numbers

$$
\eta(r)=\left|p(m) \sum_{s=r}^{r+v-1} \frac{e(s)}{p(s) p(s+1)}\right|, r=m, m+1, \ldots
$$


is begun. Forward elimination is continued further, together with the calculation of $\eta(r)$, until a value of $r$ is reached such that $\eta(r)<\varepsilon$. This value of $r$ is taken for $n$; its adequacy may be checked by taking additional terms in the infinite expansion of $y(m)-y_{n}(m)$, given by (1.1.2) with $r=m$.

In addition to being appropriate for computing intermediate solutions of (1.1.1), Olver's algorithm is suited to computing the minimal solution of a homogeneous equation. It can therefore be compared to Miller's algorithm. The advantage of Olver's algorithm over Miller's algorithm is that the latter does not have a criterion for determining automatically an appropriate value for $n$. On the other hand, if an adequate value of $n$ is known from other considerations (as, for example, from an asymptotic estimate), then Miller's algorithm may require less computational effort.

Zahar [27], Oliver $[19,20]$, and Cash [6] have considered extensions of Olver's algorithm to linear difference equations of higher order. In the present work we shali construct and analyze an algorithm which has features in common with these extensions. We shall also illustrate the algorithm by means of numerical examples. 


\subsection{Factorization of Linear Difference Equations}

Let $F$ be the field of complex numbers and let $S$ be the set of all complex-valued infinite sequences $x=(x(0), x(1), \ldots)$. Clearly $S$ is an infinite-dimensional linear space over $F$. Let $D_{\ell}$ be the set of all infinite upper-triangular band matrices of the form

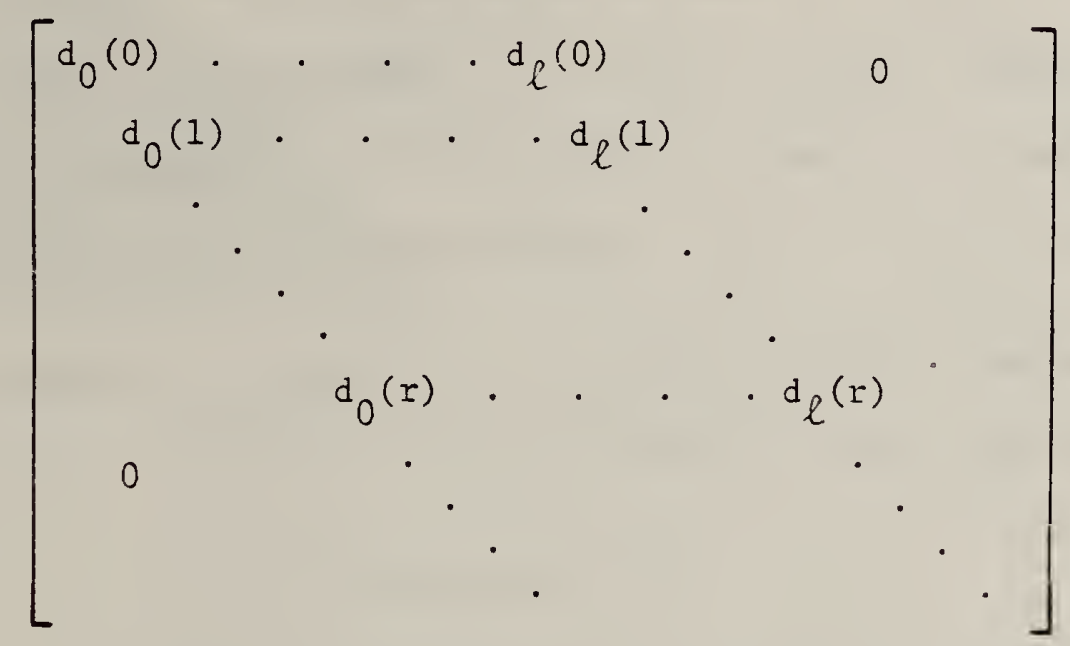

where $d_{0}, d_{1}, \ldots, d_{\ell} \in S$ and $d_{0}(r) \neq 0, d_{\ell}(r) \neq 0$ for infinitely many values of $r$. We shall also use the concise notation $\left[d_{0}, d_{1}, \ldots, d_{\ell}\right]$ to represent infinite matrices in the set $D_{\ell}$. If we regard the infinite sequences in $S$ as infinite column vectors, then an infinite sequence $D x \in S$ is defined for each $D \in D_{\ell}, x \in S$ by ordinary matrix multiplication. In fact, the terms of $D x$ are given by

$$
D x(r)=\sum_{s=0}^{\ell} d_{s}(r) x(r+s), r=0,1,2, \ldots .
$$

Clearly, each $D \in D_{\ell}$ is a linear operator on $S$ under this definition. We shall say that $D_{\ell}$ is the class of linear difference operators of order $\underline{\ell}$ on $S$.

Note that $D_{\ell}$ is defined for each integer $l \geq 0$. We pointed out in $\$ 1.1$ that intermediate solutions of Iinear difference equations 
can exist only if the order of the equation is at least two. But we are going to factor linear difference operators into a product of two lowerorder operators. Therefore, operators of order one will arise. Furthermore, the formalism to be introduced will allow even the degenerate case of operators of order zero.

If $d_{0}$ and $d_{l}$ have no terms equal to zero, we shall say the difference operator $\left[d_{0}, d_{1}, \ldots, d_{l}\right] \in D_{l}$ is nonsingular; compare $[14, \S 12.0]$. The terms of $d_{0}$ and $d_{l}$ will be called the leading and trailing coefficients of $\left[d_{0}, d_{1}, \ldots, d_{\ell}\right]$, respectively.

We shall use the notation $x^{i}$ to indicate a sequence from $S$ whose first term is $x(i)$. Thus

$$
x^{i}=(x(i), x(i+1), \ldots)
$$

The point $i$ will be called the initial point of the sequence. When we do not indicate a superscript it is to be understood that zero is the initial point of the sequence. Similarly, we shall use the notation $x^{i, n}$ to indicate the (finite) subsequence of $x^{i}$ whose final term is $x(n)$. Thus

$$
x^{i, n}=(x(i), x(i+1), \ldots, x(n))
$$

The point $n$ will be called the terminal point of the subsequence.

Similarly, for linear difference operators from $D_{\ell}$, we shall use the notation $D^{i}$ or $\left[d_{0}^{i}, d_{l}^{i}, \ldots, d_{l}^{i}\right]$ to indicate that $i$ is the initial point of each of the $\ell+1$ sequences involved in the definition of the operator. See Figure 1. When the superscript is omitted, it is understood that these initial points are all zero unless it is clear from the context (as in equation (1.2.1) below) what these injtial points are. 

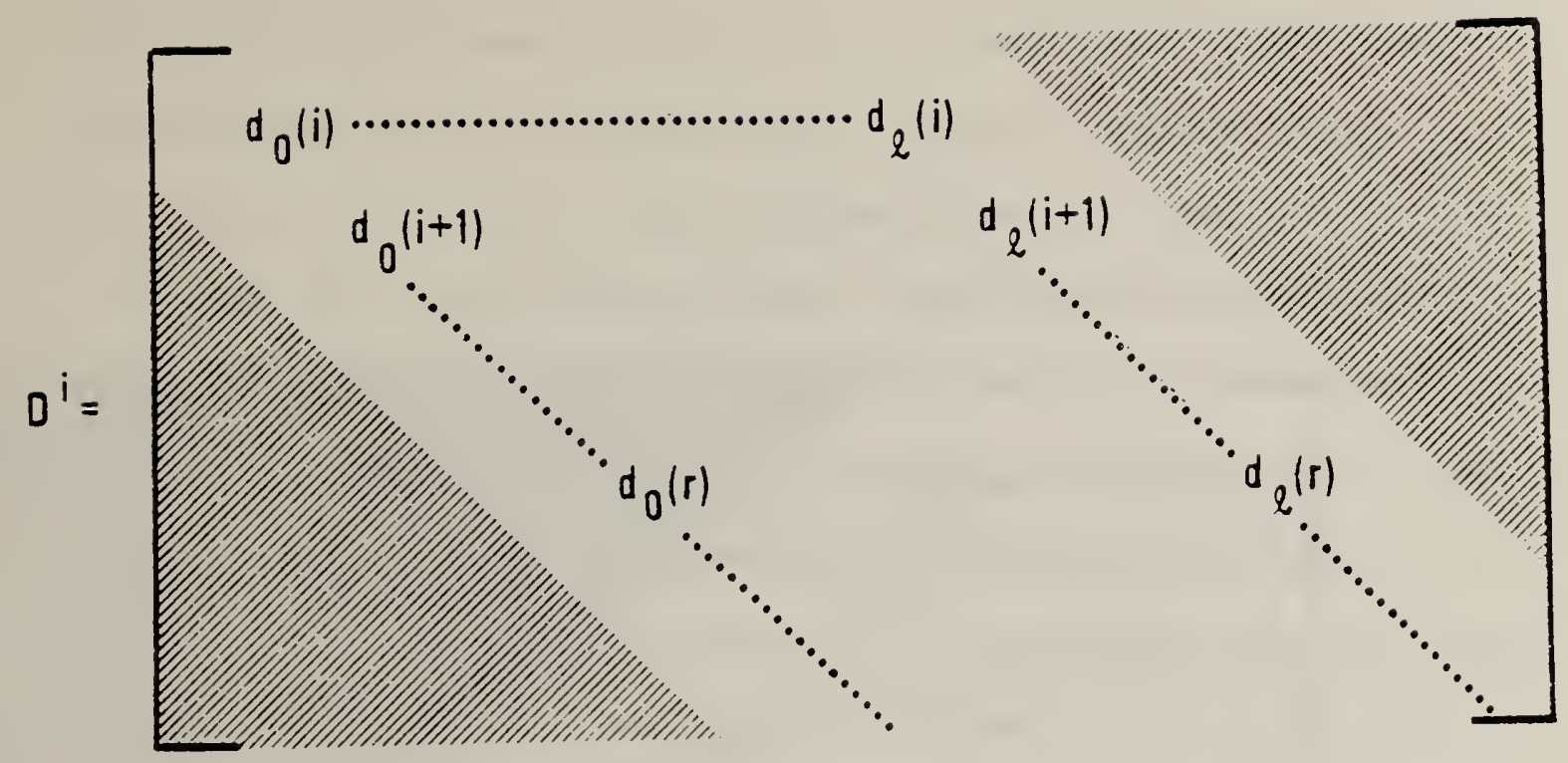

Figure 1. The operator $D^{i}$.

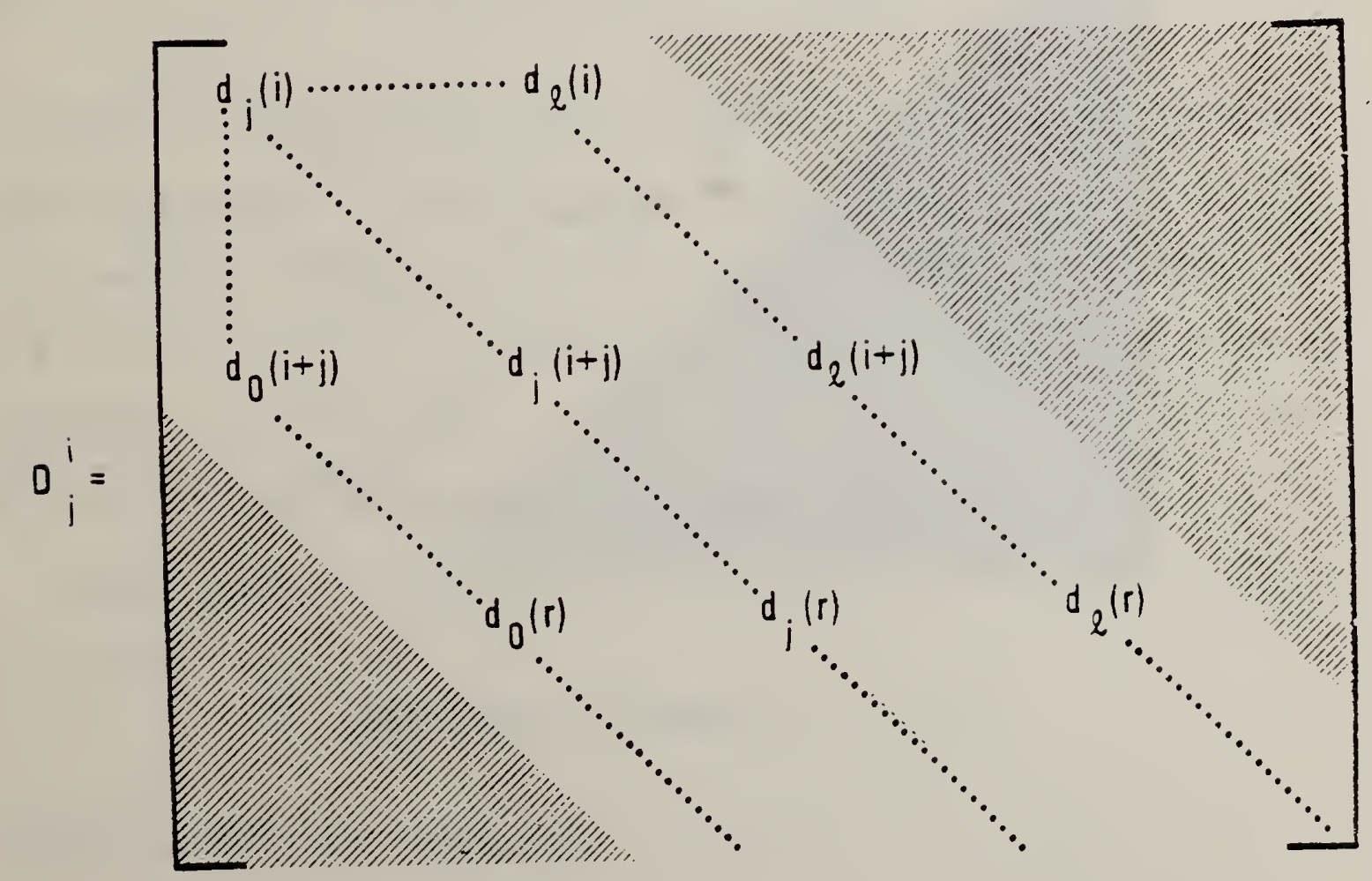

Figure 2. The matrix $D_{j}^{i}$. 


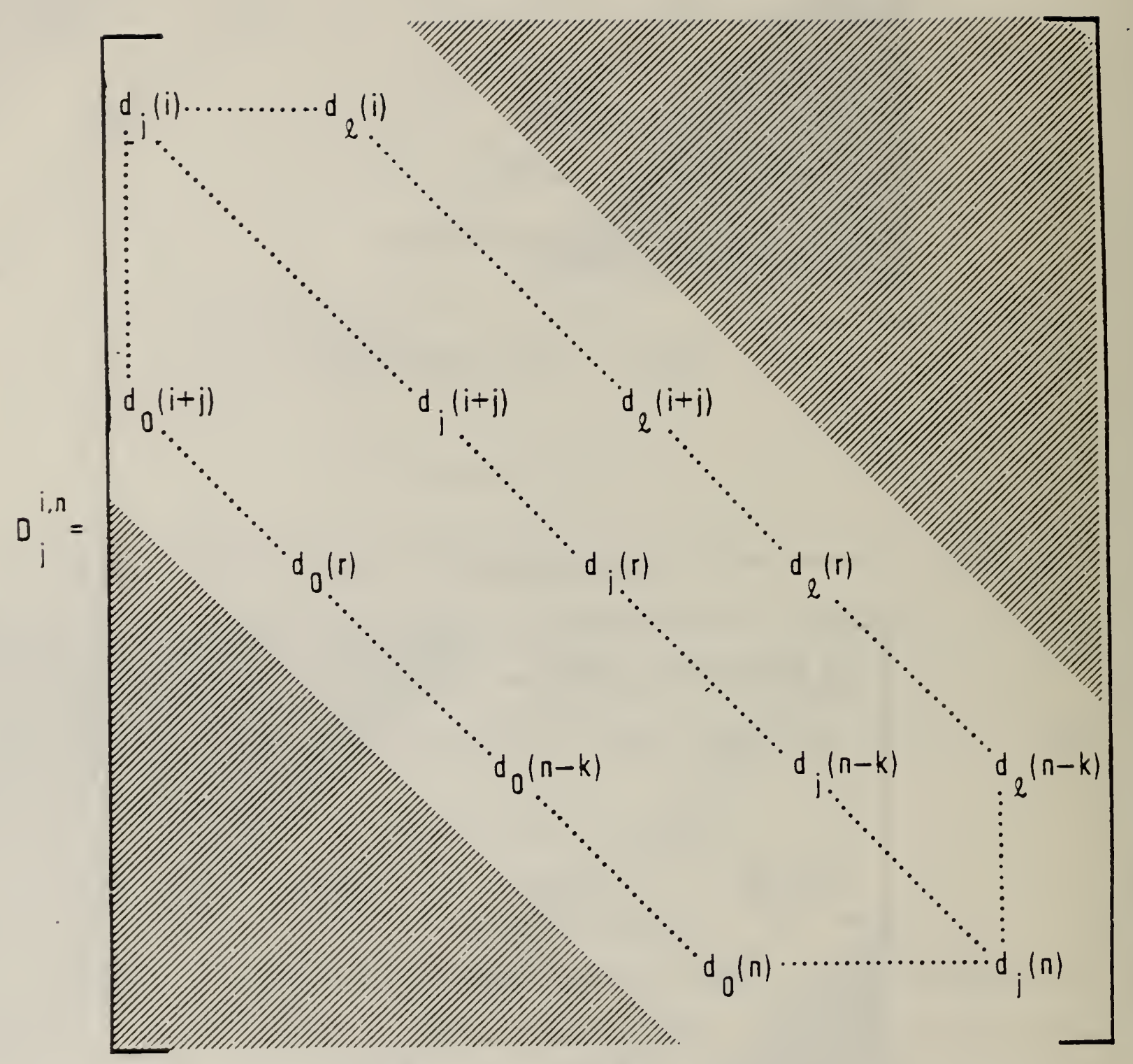

Figure 3. The matrix $D_{j}^{i, n}$. 
Two further notational conventions to be used in this thesis are $\mathrm{D}_{j}^{i}$, the infinite matrix obtained from $D^{i}$ by deleting the first $j$ columns $(0 \leq j \leq \ell)$; and $D_{j}^{i, n}$, the leading principal submatrix of order $n-i+1$ of $D_{j}^{i}$. See Figures 2 and 3 . Note that each diagonal of $\mathrm{D}_{j}^{i}$ below the principal diagonal is a subsequence of one of the sequences $d_{0}^{i}, d_{1}^{i}, \ldots, d_{j-1}^{i}$, the sequence of $d_{j}^{i}$ is on the principal diagonal, and the sequences $d_{j+1}^{i}, \ldots, d_{l}^{i}$ form the diagonals above the principal diagonal. The form of the finite matrix $D_{j}^{i, n}$ is similar, with the finite subsequence $d_{j}^{i, n}$ lying on the principal diagonal.

A linear difference equation is an equation of the form $D x=g$, where $g \in S$ and $D \in D_{l}$ are prescribed. Either $x \in S$ (or a subsequence of $x$ of the form $x^{i, n}$ ) is to be determined. This equation is equivalent to the infinite linear algebraic system

$$
\operatorname{Dx}(r)=g(r), \quad r=0,1, \ldots .
$$

Analogously to linear differential equations, auxiliary conditions are required in order to specify a unique solution. In our investigations these auxiliary conditions will consist of two finite subsequences $x^{i, i+j-1}$ and $x^{n, n+k-1}$ (or possibly only one of these) which are to be specified in advance, where $j+k=l$ and $j \geq 0, k \geq 0$. These are called the initial and terminal conditions, respectively, when $n \geq i+j$.

Let us consider the following finite boundary value problem: Determine $y^{i+j, n-1}$ such that
$(1.2 .1)$

$$
\operatorname{Dy}(r)=g(r), \quad r=i, i+1, \ldots, n-j-1
$$

together with initial conditions

$$
y(i+r)=a_{r}, \quad r=0,1, \ldots, j-1
$$


and terminal conditions

$$
y(n+r)=\beta_{r}, \quad r=0,1, \ldots, k-1
$$

are satisíied, where $D \in D_{l}, l \geq 1, g \in S, j \in\{0,1, \ldots, \ell\}, k=\ell-j$, $i \geq 0, n \geq i+j$ and $a_{0}, a_{1}, \ldots, a_{j-1}, \beta_{0}, \beta_{1}, \ldots, \beta_{k-1} \in F$. In view of $(1.2 .2)$ and $(1.2 .3)$, the finite subsequences $y^{i, i+j-1}$ and $y^{n, n+k-1}$ are known. Of course, if $j=0$ then $y^{i, i+j-1}$ is null. Similarly if $k=0$. These cases correspond to the absence of either (1.2.2) or (1.2.3). The unknown subsequence $y^{i+j, n-1}$ satisfies a linear system of algebraic equations which can be expressed in matrix terms using the notation we introduced above. The system is

$$
\begin{aligned}
& \text { (1.2.4) }
\end{aligned}
$$

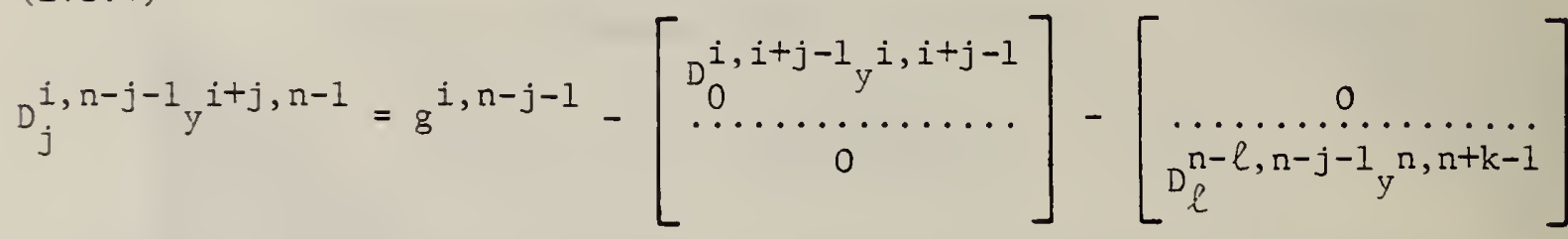

provided that $n-i-j \geq \max (j, k)$. The first partitioned column vector on the right side of $(1.2 .4)$ has $j$ entries in the upper part, and the second partitioned column vector has $k$ entries in the lower part. The length of the other column vectors appearing in $(1.2 .4)$ is $n-i-j$. This is what gives rise to the restriction $n-i-j \geq \max (j, k)$. This restriction is irrelevant for the applications we have in mind, since we shall be considering sequences of finite boundary value problems as $\mathrm{n} \rightarrow \infty$. Note that the number of zeros represented by the symbol 0 in the partitioned column vectors is $n-i-2 j$ in the first vector and $n-i-l$ in the second vector. Obviously, the partitioned column vectors reflect the influence of the boundary conditions (1.2.2) and (1.2.3).

Solution of the boundary value problem (1.2.1) to (1.2.3) is equivalent to solution of the linear system (1.2.4). Equation (1.2.4) possesses 
a unique solution if, and only if, the matrix $D_{j}^{i, n-j-1}$ is nonsingular. If in addition we assume that the difference operator $D$ is nonsingular, then the solution $y^{i, n+k-1}$ of $(1.2 .1)$ to $(1.2 .3)$ extends uniquely to $y \in S$ by forward and backward recurrence. However, nonsingularity of $D$ is not necessary for this unique extension to exist; for example, some of the leading and trailing coefficients of $D$ which appear in the finite matrix $D_{j}^{i, n-j-1}$ could be zero.

Let us consider the nonsingular triangular cases of (1.2.4). The first is given by $j=\ell$. Then (1.2.4) assumes the form

(1.2.4a) $\quad D_{l}^{i, n-\ell-1_{y} i+\ell, n-1}=g^{i, n-l-1}-\left[\begin{array}{c}D^{i, i+\ell-1}{ }^{i, i+l-1} \\ 0 \ldots \ldots \ldots \ldots \ldots . \\ 0\end{array}\right]$.

Writing out these equations in detail, we have

$$
\begin{aligned}
d_{\ell}(i) y(i+\ell) & =g(i)-\sum_{s=0}^{\ell-1} d_{s}(i) y(i+s) \\
d_{\ell-1}(i+1) y(i+\ell)+d_{\ell}(i+1) y(i+\ell+1) & =g(i+1)-\sum_{s=0}^{\ell-2} d_{s}(i+1) y(i+s+1) \\
& \cdot \\
& \cdot \\
& \cdot \\
\sum_{s=0}^{\ell} d_{s}(i+\ell) y(i+\ell+s) & =g(i+\ell) \\
& \cdot \\
\sum_{s=0} d_{s}(n-\ell-1) y(n-\ell-1+s) & =g(n-\ell-1)
\end{aligned}
$$

Since this system is lower triangular, it is solvable by determining $y(i+l)$ from the first equation, $y(i+l+1)$ from the second equation, and so on. This process is equivalent to developing $y(i+\ell), y(i+\ell+1), \ldots, y(n-1)$ from (1.2.1) by forward recurrence starting with the given values (1.2.2). 
The second triangular case is given by $j=0$. Then (1.2.4) assumes the form

$$
D_{0}^{i, n-1} y^{i, n-1}=g^{i, n-1}-\left[\begin{array}{c}
0 . \cdots \cdots \cdots \cdot \cdots \\
D_{l}^{n-l, n-1} n, n+l-1
\end{array}\right]
$$

Writing out these equations in detail, we have

$$
\begin{aligned}
\sum_{s=0}^{\ell} d_{s}(i) y(i+s) & =g(i) \\
& \cdot \\
& \cdot \\
& \cdot \\
\sum_{s=0}^{l} d_{s}(n-\ell-1) y(n-\ell-1+s) & \cdot g(n-\ell-1) \\
& \cdot \\
d_{0}(n-2) y(n-2)+d_{1}(n-2) y(n-1) & =g(n-2)-\sum_{s=2}^{l} d_{s}(n-2) y(n-2+s) \\
d_{0}(n-1) y(n-1) & =g(n-1)-\sum_{s=1}^{\ell} d_{s}(n-1) y(n-1+s)
\end{aligned}
$$

Since this system is upper triangular, it is solvable by determining $y(n-1)$ from the last equation, $y(n-2)$ from the next-to-last equation, and so on. This process is equivalent to developing $y(n-1), y(n-2), \ldots$, $y(i)$ from (1.2.1) by backward recurrence starting with the values (1.2.3). The noneriangular cases of $(1.2 .4)$ in which $0<j<\ell$ will be of greater interest to us. Our primary goal is to find a way to solve these problems by performing, in effect, a forward recurrence of order $j$ followed by a backward recurrence of order $\ell-j$.

Definition 1.2.I A nonsingular boundary value problem (1.2.4), or equivalently (1.2.1) to (1.2.3), having initial point $i$, terminal point $n$, number cf initial conditions $j$ and difference operator $D=\left[d_{0}, d_{1}, \ldots, d_{\ell}\right] \in D_{\ell}$, will be called factorizable provided there 
exist difference operators $A^{i}=\left[a_{0}^{i}, a_{1}^{i}, \ldots, a_{j}^{i}\right]$ of order $j$ and

$B^{i+j}=\left[b_{0}^{i+j}, b_{1}^{i+j}, \ldots, b_{k}^{i+j}\right]$ of order $k=l-j$ such that

$(1.2 .5)$

$$
D_{j}^{i, n-j-1}=A_{j}^{i, n-j-1} B_{0}^{i+j, n-1}
$$

If in addition the sequences $a_{j}^{i}$ and $b_{0}^{i+j}$ are free of zeros and the infinite matrix factorization

$$
D_{j}^{i}=A_{j}^{i} B^{i+j}
$$

is valid, then we shall say that the difference operatnr $D$ is $(i, j)$ factorizable.

Several remarks may be made here. Note that $D_{j}^{i, n-j-1}$ and $D_{j}^{i}$ are band matrices each having total bandwidth $l+1$, lower bandwidth $j$, and upper bandwidth $k$. A nonsingular and factorizable boundary value problem is one for which a finite matrix factorization of $D_{j}^{i, n-j-1}$ exists such that the left factor is lower triangular with total bandwidth $j+1$ and the right factor is upper triangular with total bandwidth $k+1$. The difference operators $A^{i}$ and $B^{i+j}$ may be arbitrary as long as they satisfy the condition (1.2.5), since (1.2.5) involves only a finite submatrix of each of $A^{i}$ and $B^{i+j}$. Equation (1.2.5) is illustrated in Figure 4. Since $D_{j}^{i, n-j-1}$ is nonsingular, both $A_{j}^{i, n-j-1}$ and $\mathrm{B}_{0}^{i+j, n-1}$ are nonsingular. Thus the entries on the principal diagonals of $A_{j}^{i, n-j-1}$ and $B_{0}^{i+j, n-1}$ are all nonzero.

Now suppose the difference operator $D \in D_{l}$ is $(i, j)$ - factorizable; it need not be a nonsingular operator. Because of the triangular forms of $A_{j}^{i}$ and $B^{i+j}$, equation (1.2.6) implies equation (1.2.5) for every $n \geq i+j+1 . \quad$ In words, every leading principal submatrix of $D_{j}^{i}$ is equal to the product of the corresponding leading principal submatrices of $A_{j}^{i}$ and $B^{i+j}$. The assumption that the subsequences $a_{j}^{i}$ and $b_{0}^{i+j}$ are free of zeros is equivalent to assuming that the entries on the 

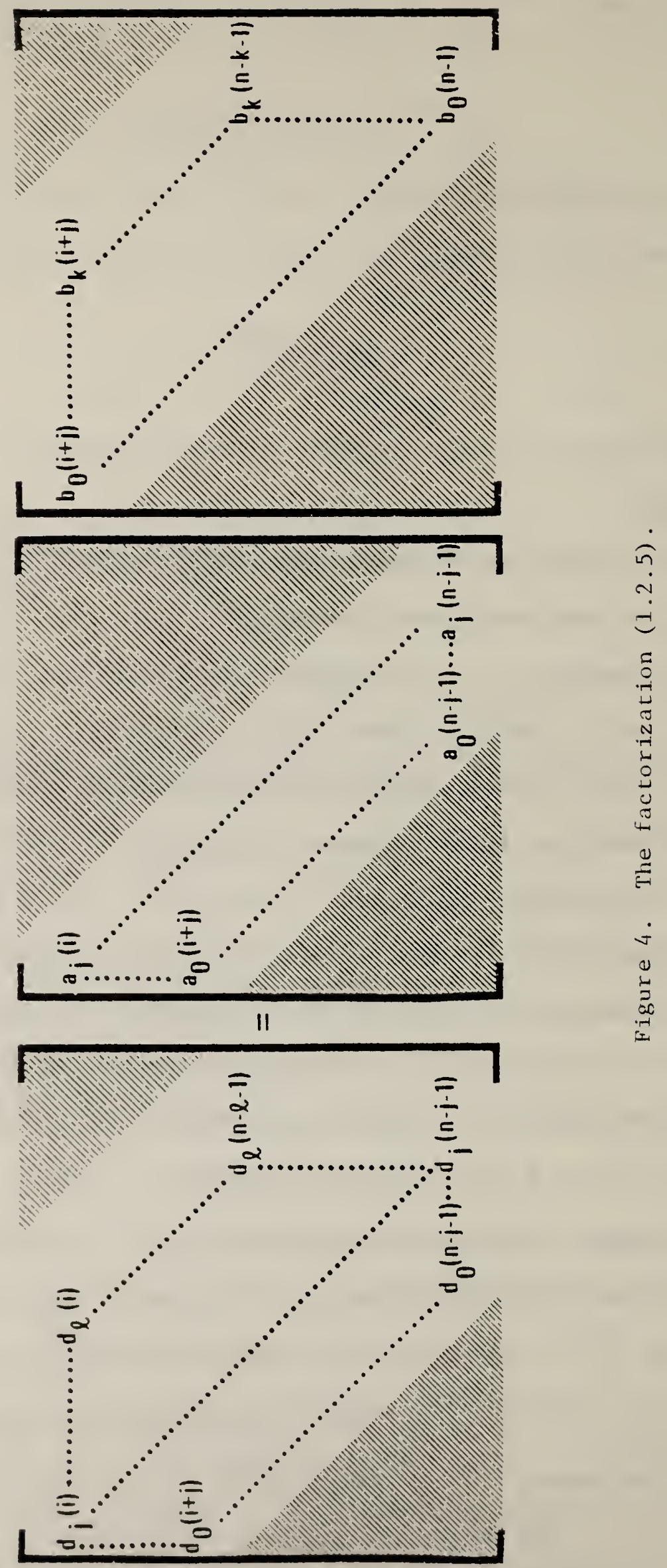
principal diagonals of the infinite matrices $A_{j}^{i}$ and $B^{i+j}$ are all nonzero. It foliows that every leading principal submatrix of $D_{j}^{i}$ is nonsingular. Thus we have proved the following theorem:

Theorem 1.2.1 Let $D \in D_{l}$ be $(i, j)$ - factorizable. Then, for every $n \geq i+j+\max (j, \ell-j)$, the boundary value problem (1.2.4) having difference operator $D$, initial point $i$, number of initial conditions $j$ and terminal point $\mathrm{n}$ is nonsingular and factorizable.

The next theorem shows it is possible to obtain a solution to a factorizable boundary value problem, in theory at least, by performing an appropriate forward recurrence followed by an appropriate backward recurrence.

Theorem 1.2.2 Let $(1.2 .4)$ be a nonsingular and factorizable boundary value problem with initial point $i$, number of initial conditions $j$, terminal point $n$ and difference operator $D \in D_{l}$. If $A^{i}$ and $B^{i+j}$ are difference operators of order $j$ and $k=l-j$, respectively, such that (1.2.5) is valid, then the solution of (1.2.4) is identical to the solution of

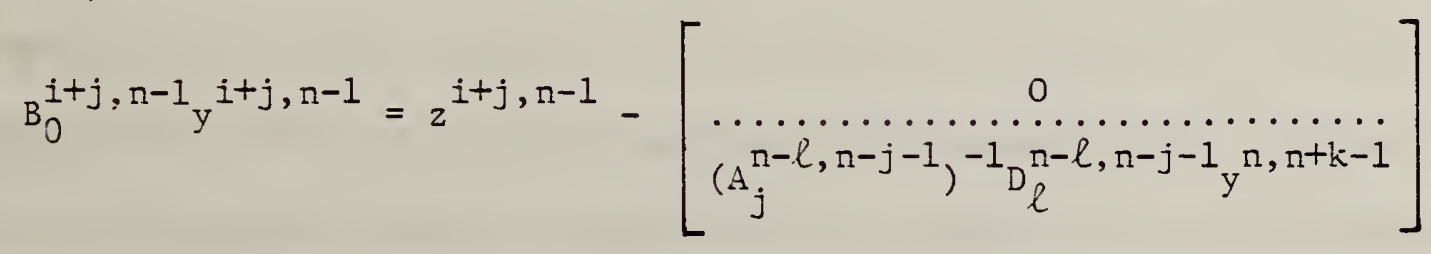

where $z^{i+j, n-1}$ is the solution of

$$
A_{j}^{i, n-j-1} z^{i+j, n-1}=g^{i, n-j-1}-\left[\begin{array}{c}
D^{i, i+j-1} y, i+j-1 \\
0 \ldots \ldots \ldots \ldots \\
0
\end{array}\right] .
$$


Proof The matrices $A_{j}^{i, n-j-1}$ and $B_{0}^{i+j, n-1}$ are nonsingular because $J_{j}^{i, n-j-1}$ is nonsingular; see (1.2.5). Therefore, the diagonal elements of $A_{j}^{i, n-j-1}$ and $B_{0}^{i+j, n-1}$ (which are triangular matrices) are nonzero and $(1.2 .7)$ and $(1.2 .8)$ are nonsingular linear systems. Furthermore, $A_{j}^{n-\ell, n-j-1}$ is nonsingular because it is a principal submatrix of $A_{j}^{i, n-j-1}$. Therefore the right side of $(1.2 .7)$ is unambiguously defined. Now, premultiplying $(1.2 .7)$ by $A_{j}^{i, n-j-1}$ and using (1.2.5) and $(1.2 .8)$, we obtain $D_{j}^{i, n-j-1} y^{i+j, n-1}=$

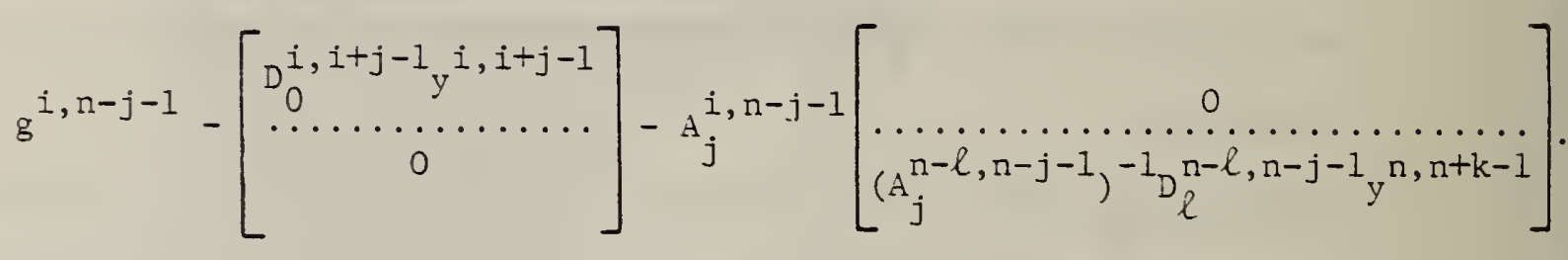

Consider the last product on the right side of this equation. Its block structure is

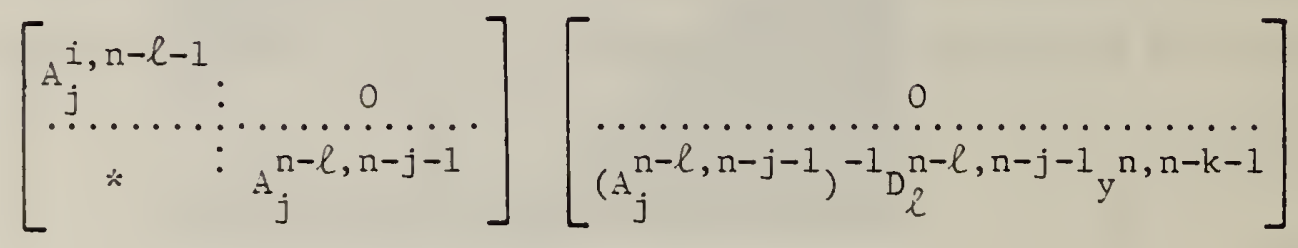

where 0 in the matrix denotes a zero submatrix with $n-i-l$ rows and $k(=\ell-j)$ columns, 0 in the vector denotes a zero subvector of length $n-i-l$, and $*$ in the matrix denotes a submatrix which needs no further identification here. Therefore, the product in question is equal to the vector

$$
\left[\begin{array}{c}
0 \\
D_{l}^{n-l, n-j-1}, \ldots \ldots \cdots \\
n, n+k-1
\end{array}\right]
$$


Substitution into the equation and comparison with (1.2.4) completes the proof.

An important remark is that if $(1.2 .6)$ is valid, i.e. if $D$ is $(i, j)-$ factorizable with left factor $A^{i}$ and right factor $B^{i+j}$, then

$$
D_{\ell}^{n-\ell, n-j-1}=A_{j}^{n-\ell, n-j-1} B_{k}^{n-k, n-1}
$$

In this case $B_{k}^{n-k, n-1}$ may be written in place of $\left(A^{n-\ell, n-j-1}\right)^{-1}$ $D^{n-\ell, n-j-1}$ in (1.2.7). The truth of this remark is apparent from Figure 4. The next theorem gives necessary and sufficient conditions for a difference operator to be $(i, j)$ - factorizable. The proof also shows that $(i, j)$ - factorizations may be constructed by Gaussian elimination in natural order, i.e., without any row or column interchanges. Theorem 1.2.3 Let $\mathrm{D} \in \mathrm{D}_{\ell}$. Then

i) A nonsingular boundary value problem having initial point $i$, number of initial conditions $j$ and terminal point $n$ is factorizable if every leading principal submatrix of $D_{j}^{i, n-j-1}$ is nonsingular.

ii) $D$ is $(i, j)$ - factorizable if every leading principal submatrix of $D_{j}^{i}$ is nonsingular. Furthermore, this condition is necessary if $D$ is nonsingular.

Proof Our approach is to apply Gaussian elimination [10, $\$ 2.1]$ without any row or column interchanges. Because Gaussian elimination is so familiar, we merely sketch the proof. The proof of (i) is contained in the first $n-i-j$ stages of the proof of (ii); therefore we supply only the proof of (ii). We begin by proving sufficiency of the condition. The first row of $D_{j}^{i}$ is used to annihilate every nonzero element below the first element in the first column. This is possible because the pivot element, which is the leading principal minor of order one, is nonzero. Because of the band structure, only the $j$ rows immediately follow- 
ing the first row are affected. Also (again because of the band structure) the trailing coefficient in each of these rows is not changed. The multipliers used in these annihilations go into positions 2 through $j+1$ of the first column of the lower triangular factor $A_{j}^{i}$. The first element is unity and every other element of the first column of $A_{j}^{i}$ is zero. This completes the first stage.

Now suppose the $(r-1)$ st stage has been completed. Let the notation $\bar{D}_{j}^{i}$ denote the transformation of the original $D_{j}^{i}$ resulting from the first $r-1$ stages. The leading principal submatrix of $\bar{D}_{j}^{i}$ of order $r$ is upper triangular, having resulted in effect from the corresponding principal submatrix of the original $D_{j}^{i}$ by Gaussian elimination in natural order. Its diagonal elements are all nonzero, by hypothesis. Therefore the pivotal element is nonzero and the $r-t h$ row of $\bar{D}_{j}^{i}$ may be used to annihilate every nonzero element below the $r$-th element in the $r$-th column. Only the $j$ rows immediately following the $r$-th row are affected, and the trailing coefficient in each of these rows is not changed. The multipliers used in this annihilation go into positions $r+1$ through $r+j$ of the $r$-th column of $A_{j}^{i}$; unity goes into the $r$-th position, and zeros go into every other position. This completes the $r$-th stage.

Let $B^{i+j}$ be the infinite upper triangular matrix which results from the full sequence of transformations on $D_{j}^{i}$. By our construction, both $A_{j}^{i}$ and $B^{i+j}$ have the bandwidths required by $(i, j)$ - factorizability. Also by our construction, formally multiplying $A_{j}^{i}$ on the right by $B^{i+j}$ results in $D_{j}^{i} ;$ compare $(1.2 .6)$.

In order to prove necessity of the condition in part (ii), note that the factorization produced by the foregoing process is such that leading and realing coefficients of both $A_{j}^{i}$ and $B^{i+j}$ are nonzero if $D$ is 
nonsingular. Therefore, no row or column interchanges could be made without widening the bandwidth. Therefore, Gaussian elimination in natural order is the only possibility for arriving at the factorization. But Gaussian elimination in natural order is possible only if every leading principal minor is nonzero. This completes the proof. I-

Examination of the proof of Theorem 1.2 .3 shows that a unique (i,j)factorization does not exist, since a different scaling of the rows is possible. This would result in the appearance of entries on the principal diagonal of $\mathrm{A}_{j}^{i}$ that are other than unity. In fact, the scaling used in the $(0,1)$-factorization in 0lver's second-order algorithm put the sequence $(p(2), p(3), \ldots)$ on the principal diagonal of the right factor; compare $\$ 1.1$. Thus the principal diagonal of the left factor will contain entries other than unity, in general. 


\subsection{Areliminary Development of the Algorithm}

Let $D \in D_{l}$. It is clear that an extension of Olver's algorithm for computing a particular solution $y$ of a given difference equation $D y=g$ should involve the posing of a boundary value problem whose solution $y_{n}$ is an approximation of $y$ over some finite subsequence $y^{i, m}$. The boundary value problem will have some number $j \geq 0$ of initial conditions (depending on the particular solution being sought). These will consist of a subsequence of $j$ consecutive values of $y$. The terminal conditions of the boundary value problem will be $y_{n}(n)=y_{n}(n+1)$ $=\cdots=y_{n}(n+k-1)=0$, where $k=l-j$.

The first task is to decide how to choose $j$. A necessary prerequisite is a classification of possible solution types when the operator is of order higher than two. Oliver [19] provided an early extension of Olver's algorithm. He also provided the following definition of dominance. Suppose we are given two sequences $a, b \in S$. Then $b$ dominates $a$ if there exists some $i \geq 0$ such that

$$
|b(r+1) / b(r)|>|a(r+1) / a(r)|
$$

for all $r \geq i$. Oliver considered only those difference operators for which a system of complementary solutions could be found such that the complementary solutions are linearly ordered under this definition of dominance. Furthermore, he considered only those particular solutions of the given linear difference equation which could be compared to each of the complementary solutions. But not all possible particular solutions can be so compared; for example, no particular solution which has an infinite subsequence of zeros can play the role of either $a$ or $b$ in $(1.3 .1)$ 
In $\$ 1.1$, we adopted a different definition of dominance: b

dominates a if

$$
\lim _{r \rightarrow \infty} \frac{a(r)}{b(r)}=0
$$

This differs from Oliver's definition (which is equivalent to requiring that ultimately $|a(r) / b(r)|$ be monotonically decreasing). Another difference is that the limit of $|a(r) / b(r)|$ under 0liver's definition may be greater than zero, as the example $a(r)=1+1 / r$ and $b(r)=1$ immediately shows.

Let $D \in D_{l}$. The set $K(D)=\{x \in S \mid D x=0\}$ will be called the kernel of $D$. Obviously, $K(D)$ is a linear subspace of $S$. Let us suppose that $K(D)$ has dimension $l$, so that there exist $l$ 1inearly independent sequences $x_{1}, x_{2}, \ldots x_{l} \in K(D)$ which span $K(D)$. A sufficient condition for this to be the case is that $D$ be nonsingular; compare $[14, \$ 12.1]$. We shall say that the set $x_{1}, x_{2}, \ldots, x_{\ell}$ forms a basis for $D$.

Definition 1.3.1 We shall say that $D \in D_{\ell}$ is totally separable (as $r \rightarrow \infty)$ provided there exists a basis $y_{1}, y_{2}, \ldots, y_{2}$ for $D$ such that $y_{s+1}$ dominates $y_{s}$ in the sense of (1.3.2) for each $s, 1 \leq s<l$. Any basis which satisfies the conditions of Definition 1.3 .1 will be called totally ranked. Such a basis is not unique because $y_{1}$, for example, could be added to each of $y_{2}, \ldots y_{\ell}$ and the resulting basis would also be totally ranked.

For each $s, 1 \leq s \leq \ell$, let $K_{s}$ denote the linear subspace of $S$ which is spanned by $y_{1}, y_{2}, \ldots, y_{s}$. In particular, $K_{\ell}=K(D)$. It seems obvious, and it will be proved in a more general setting in Chapter 2, that every totally ranked basis generates the same subspaces. The subspaces satisfy 


$$
K_{1} \subset K_{2} \subset \cdots \subset K_{2}=K(D) .
$$

Each set inclusion is proper, since the dimension of $K_{s}$ is $s$. This ascending chain of subspaces induces an obvious classification of the solutions of $D y=0$ into $l$ distinct types. However, we are interested in classifying the solutions of $D y=g$ for nonzero, and indeed, arbitrary $g \in S$. Therefore, we Erame the definition so as to cover all cases: Definition 1.3 .2 Let $D \leqslant D_{\ell}$ be totally separable with totally ranked basis $y_{I}, y_{2}, \ldots, y_{l}$. Then, for each $y \in S$, we shall say that the type of $y$ (with respect to $D$ ) is $s$, where $0<s<\ell$, and we shall write type $(y)=s$, provided that

$$
\lim _{r \rightarrow \infty} \frac{y(r)}{y_{s+1}(r)}=0
$$

and

$$
\infty \geq \limsup _{r \rightarrow \infty}\left|\frac{y(r)}{y_{S}(r)}\right|>0 .
$$

Furthermore, if $(1.3 .3)$ is true for $s=0$, we define type(y) $=0$, and if $(1.3 .4)$ is true for $s=l$, we define type(y) $=\ell$.

The type of each $y \in S$ is the same regardless of which totally ranked basis is used. This will be proved for a more general classification of sequences in Chapter 2. Thus we are justified in defining the type with respect to $D$ rather than with respect to a particular cotally ranked basis.

The conditions for Olver's algorithm [21] suggest that Definitions 1.3.1 and 1.3 .2 are appropriate for an extension of the algorithm to arbitrary order; see also Cash [6]. But it would be desirable to have a classification scheme which is applicable, for example, to every difference 
equation with constant coefficients. Not every constant-coefficient operator is totally separable, however. In the next chapter we shall introduce a more general class of difference operators (separable operators) which does include every constant-coefficient operator. Incidentally, the class of difference equations considered by Oliver [19] includes every constant-coefficient equation, since the standard basis (see $\$ 2.1$ below) is linearly ordered under his definition of dominance. Before stating the next theorem, we explain what we mean by matrices and determinants associated with the name Casorati. If $x_{1}, x_{2}, \ldots, x_{S} \in S$ are arbitrary sequences, then for each $r \geq 0$ the $s \times s$ matrix

$$
\left[\begin{array}{cccc}
x_{1}(r) & x_{2}(r) & \ldots & x_{s}(r) \\
x_{1}(r+1) & x_{2}(r+1) & \ldots & x_{s}(r+1) \\
\vdots & \vdots & & \vdots \\
x_{1}(r+s-1) & x_{2}(r+s-1) & \ldots & x_{s}(r+s-1)
\end{array}\right]
$$

will be called a Casorati matrix. Let $\mathrm{X}=\left\{\mathrm{x}_{1}, \mathrm{x}_{2}, \ldots, \mathrm{x}_{\mathrm{s}}\right\}$. We shall use the concise notation $[X](r)$ to denote the Casorati matrix above. Furthermore, $|x|(r)$ will denote the Casoratian (of $x$ at $r$ ), defined by

$$
|x|(r)=\operatorname{det}[X](r), \quad r=0,1, \ldots .
$$

If $D \in D_{\ell}$ is nonsingular, Casorati's theorem $[14, \$ 12.11]$ states that $x_{1}, x_{2}, \ldots, x_{l}$ forms a basis for $D$ if, and only if, $|x|(r) \neq 0$ for all r .

Theorem 1.3.1 Let $y$ be a solution of the difference equation $D y=g$, where $D \in D_{l}$ has a totally ranked basis $Y=\left\{y_{1}, y_{2}, \ldots, y_{l}\right\}$. Let 
$j=$ type(y). Then $y$ is uniquely determined by its values $y(i), y(i+1)$, $\ldots, y(i+j-1)$ provided that $i \geq 0$ is a point at which the leading principal minor of order $j$ of $|Y|(i)$ is nonzero.

Proof Assume $j=0$; then the condition on the Casoratian is vacuous and we must show $y$ is uniquely determined without knowledge of any of its values. Suppose $z \neq y$ is another solution of type zero; then there exist unique scalars $a_{1}, a_{2}, \ldots, a_{l}$ (not all zero) such that

$$
y(r)=z(r)+a_{1} y_{1}(r)+\cdots+a_{\ell} y_{\ell}(r), \quad r=0,1, \ldots
$$

Let $\alpha_{t}$ be the nonzero scalar of highest subscript. Dividing through by $y_{t}(r)$ and allowing $r \rightarrow \infty$, we see that $a_{t}=0$, which is a contradiction. Next, assume $j>0$. Suppose $z \neq y$ is another solution of type $j$ such that

$$
z(i+r)=y(i+r), \quad r=0,1, \ldots, j-1
$$

There exist unique scalars $a_{1}, a_{2}, \ldots, a_{l} \in F$ (not all zero) such that

$$
y(r)=z(r)+a_{1} y_{1}(r)+\cdots+a_{\ell} y_{\ell}(r), \quad r=0,1, \ldots .
$$

It is proved easily that each of $a_{j+1}, \ldots, a_{l}$ is zero by a method similar to that of the previous paragraph. The remaining scalars are determined by the linear system

$$
a_{1} y_{1}^{i, i+j-1}+\cdots+a_{j} y_{j}^{i, i+j-1}=0
$$

(where 0 stands for a column vector of $j$ zeros and we have used the notation developed in $\$ 1.2)$. This system is nonsingular by the assumption on the Casoratian; therefore, $a_{t}=0, t=1,2, \ldots, j$, which is a 
Theorem 1.3.1 confirms the adequacy of the selection of $j$ initial conditions in the approximating boundary value problem, where $j=$ type $(y)$. In $\{3.3$ we will demonstrate that stability considerations demand exactly $j$ initial conditions, in general, for a solution of type $j$.

Now let us suppose that a value of $j$ has been selected, where $j \geq \operatorname{type}(y)$. Since $y_{n}^{i, i+j-1}=y^{i, i+j-1}$ and $y_{n}^{n, n+k-1}=0$, the linear system to be solved is

(1.3.5) $D_{j}^{i, n-j-1} y_{n}^{i+j, n-1}=g^{i, n-j-1}-\left[\begin{array}{c}D_{0}^{i, i+j-1} y^{i, i+j-1} \\ \cdots \ldots \ldots \ldots . . \\ 0\end{array}\right]$;

compare (1.2.4). The value of $\mathrm{n}$ is to be found in the course of the computation. In view of Theorem 1.3.1, $y$ is the unique solution of the infinite linear system

$$
D_{j}^{i} y^{i+j}=g^{i}-\left[\begin{array}{c}
D_{0}^{i, i+j-1} y^{i, i+j-1} \\
0 \ldots \ldots \ldots \ldots
\end{array}\right]
$$

We assume that $D$ is $(i, j)$-factorizable, $i . e$. , that linear difference operators $A^{i} \in D_{j}$ and $B^{i+j} \in D_{l-j}$ exist such that $D_{j}^{i}=A_{j}^{i} B^{i+j}$. As shown in the proof of Theorem 1.2.3, successive rows of this factorization, and indeed of the triangularization of the infinite system (1.3.6), may be generated indefinitely by the forward stage of Gaussian elimination without pivoting. The stability of this process, also, will be discussed in $\$ 3.3$.

In consequence of Theorems 1.2 .1 and 1.2 .2 and the terminal conditions $y_{n}^{n, n+k-1}=0$, the solution of (1.3.5) may be obtained as the solution of $(1.3 .7)$

$$
B_{0}^{i+j, n-1} y_{n}^{i+j, n-1}=z^{i+j, n-1}
$$


where $z^{i+j, n-1}$ is a finite subsequence of the solution of

$$
A_{j}^{i} z^{i+j}=g^{i}-\left[\begin{array}{c}
D^{i, i+j-1}{ }^{i, i+j-1} \\
0 \ldots \ldots \ldots \ldots \ldots \\
0
\end{array}\right] \text {. }
$$

Comparison of (1.3.8) and (1.3.6) and reference to the identity $D_{j}^{i}=A_{j}^{i} B^{i+j}$ show that

$$
B^{i+j} y^{i+j}=z^{i+j} .
$$

Thus (1.3.7) represents the finite linear system that results from (1.3.9) by truncation at the $(n-i-j)$ th row and column. Since $B^{i+j}$ and $z^{i+j}$ are generated by Gaussian elimination without pivoting, the solution of (1.3.5) for any $\mathrm{n}$ is easily programmed for an automatic digital computer. The algorithm comprises two stages: (i) forward elimination (solution of (1.3.8)); (ii) back substitution (solution of (1.3.7)). The forward elimination san be continued arbitrarily far. The back substitution is numerically equivalent to backward recurrence, and proceeds antil we return to the initial point $i$.

The question of the stability of the back substitution is easily settled. For totally separable operators we have the following theorem: Theorem 1.3 .2 Let $D \in D_{\ell}$ have a totally ranked basis $Y=\left\{y_{1}, y_{2}, \ldots, y_{\ell}\right\}$. Suppose $i \geq 0$ and $j \leqslant\{0,1, \ldots, \ell-1\}$ are such that $D$ is $(i, j)-$ factorizable and the leading principal minor of order $j$ of $|Y|(i)$ is nonzero. If $D_{j}^{i}=A_{j}^{i} B^{i+j}$ is an $(i, j)$ - factorization of $D$, then $B^{i+j}$ is totally separable. Furthermore, if $z \in S$ has rype $j$ or less with respect to $D$, then $z^{i+j}$ has type zero with respect to $B^{i+j}$. Proof Suppose $j=0$. Then $A_{j}^{i}$ is a diagonal matrix with nonzero elements on the diagonal; see Definition 1.2.1. For each $y \in Y$ we have 


$$
\begin{aligned}
D^{i} y^{i}=A^{i} B^{i} y^{i}= & 0, \text { i.e. } \\
& \sum_{s=0}^{\ell} a_{0}(r) b_{s}(r) y(r+s)=0, \quad r=i, i+1, \ldots .
\end{aligned}
$$

Therefore,

$$
\sum_{s=0}^{l} b_{s}(r) y(r+s)=0, r=i, i+1, \ldots
$$

which proves $\left\{y^{i} \mid y \leq Y\right\}$ is a totally ranked basis for $B^{i}$. It follows that $z^{i}$ has type zero with respect to $B^{i}$ whenever $z$ has type zero with respect to $D$.

Suppose $0<j<\ell$. Let $U=\left\{y_{1}, y_{2}, \ldots, y_{j}\right\}$. Since $|U|(i) \neq 0$ it is possible to find a linear combination $x$ of $y_{1}, y_{2}, \ldots, y_{j}$ such that $x(i), x(i+1), \ldots, x(i+j-1)$ take on any prescribed set of values. Therefore, appropriate linear combinations $x_{s}, s=j+1, j+2, \ldots, 2$, can be found such that $y_{s}(r)+x_{s}(r)$ vanish for $r=i, i+1, \ldots, i+j-1$. Let $v_{s-j}=y_{s}+x_{s}, s=j+1, j+2, \ldots, l$. It is easy to verify that $\left\{y_{1}, \ldots, y_{j}, v_{1}, \ldots, v_{k}\right\}$ is a totally ranked basis for $D$. Now let $v \in\left\{v_{1}, v_{2}, \ldots, v_{k}\right\}$. In view of Theorems 1.2 .1 and 1.2 .2 , the remark following Theorem 1.2.2, and the facts that $D v=0$ and $v(i+r)=0$ for $r=0,1, \ldots, j-1$, we conclude that $v^{i+j, n-1}$ satisfies

$$
B_{0}^{i+j, n-1} v_{v}^{i+j, n-1}=-\left[\begin{array}{c}
\ldots \ldots \\
\cdots \cdots \ldots . . \cdots \\
B_{k}^{n-k, n-1} n, n+k-1
\end{array}\right]
$$

for every $n \geq i+j+\max (j, k) ;$ compare equation (1.2.7). On letting $n \rightarrow \infty$ this proves $B^{i+j} v^{i+j}=0$. Thus $\left\{v_{1}^{i+j}, v_{2}^{i+j}, \ldots, v_{k}^{i+j}\right\}$ is a totally ranked basis for $B^{i+j}$. Finally, let $z$ be of type $j$ or less with respect to $D$. Let $s \in\{1,2, \ldots, k\}$. Then 


$$
\frac{z(r)}{v_{S}(r)}=\frac{z(r)}{y_{j+s}(r)\{1+o(I)\}} \rightarrow 0 \text { as } r \rightarrow \infty
$$

which shows that $z^{i+j}$ has type zero with respect to $B^{i+j}$.

In consequence of Theorem 1.3 .2 and our remarks in $\mathfrak{\jmath} 1.1$, when $j \geq$ type $(y)$ the back substitution stage of the algorithm is stable, at least for all $r$ in a sufficiently large range.

It remains to determine the appropriate value of the truncation parameter $\mathrm{n}$. It is necessary to introduce Green's formula for linear difference operators; see, e.g., Miller [13]. We apply Green's formula not to the operator $D$ but to the operator $B^{i+j}$. Our development is essentially the same as that of Cash [6].

Let $B=\left[b_{0}, b_{1}, \ldots, b_{k}\right] \in D_{k}$. The infinite upper triangular matrix $\hat{B}=\left[\hat{b}_{0}, \hat{b}_{1}, \ldots, \hat{b}_{n}\right] \in D_{k}$ which is obtained from $B$ by deleting the first $k$ columns and transposing the result, i.e. whose entries are defined by

$$
\hat{b}_{s}(r)=b_{k-s}(r+s)
$$

is a linear difference operator of order $k$. We shall call $\hat{B}$ the operator which is adjoint to $\underline{B}$, or more briefly, the adjoint operator of $\underline{B}$. An operator and its adjoint satisfy a number of elementary properties which are easily verified from the definition. Among these are

$$
\begin{aligned}
\hat{B}^{p} & =\left(B_{k}^{p}\right)^{\operatorname{Tr}} \\
\hat{B}_{0}^{p \cdot q} & =\left(B_{k}^{p, q}\right)^{\operatorname{Tr}} \\
\hat{B}_{k}^{p} & =\left(B^{p+k}\right)^{\operatorname{Tr}}
\end{aligned}
$$


$(1.3 .14)$

$$
\hat{B}_{k}^{p, q}=\left(B_{0}^{p+k, q+k}\right)^{T r},
$$

valid for $q \geq p$ and $p=0,1,2, \ldots$.

Now take any $u, v \in S$ and $B \in D_{k}$, and assume $k \geq 1$. Green's formula is the identity

$$
\begin{aligned}
\sum_{r=p}^{q-1}\{v(r) B u(r)-u(r+k) \hat{B} v(r)\} \\
=\left(v^{p, p+k-1}\right) \operatorname{Tr}_{B} p, p+k-1{ }_{u}^{p}, p+k-1 \\
\\
-\left(v^{q, q+k-1}\right)^{\operatorname{Tr} r_{B}^{q}, q+k-1} u_{u}^{q, q+k-1}
\end{aligned}
$$

valid for $q \geq p+k, p \geq 0$. Green's formula may be verified by expressing the left side of (1.3.15) in the form

$$
\left(v^{p, q-1}\right)^{T r}(B u)^{p, q-1}-\left(u^{p+k, q+k-1}\right)^{T r}(\hat{B} v)^{p, q-1}
$$

and reducing this expression with the aid of the definition (1.3.10) and the identities (1.3.11) - (1.3.14).

Theorem 1.3.3 Let $D_{j}^{i}=A_{j}^{i} B^{i+j}$ be an $(i, j)$-factorization of $D \in D_{l}$, where $D$ is totally separable and $j<\ell$. Let $y$ be a solution of Dy $=g$, where type $(y) \leq j$ and $g \in S$. Let $y_{n}^{i+j, n-1}$ and $z^{i+j}$ be the corresponding solutions of (1.3.5) and (1.3.8). Also, let $s$ be a fixed integer satisfying $i+j+k-1 \leq s<n$ and $w_{s}^{s+1}=\left(w_{s}(s+1)\right.$, $\left.w_{s}(s+2), \ldots\right)$ be the solution of

$$
\hat{B}_{k}^{s-k+1}{ }_{w}^{s+1}=-\left[\begin{array}{c}
\hat{B}_{s}^{s-k+1, s} s-k+1, s \\
0 \ldots \ldots . . .1 . \cdots \\
0
\end{array}\right]
$$

where $k=\ell-j$ and 
$(1.3 .17)$

$$
w_{s}^{s-k+1, s}=(0,0, \ldots, 0,1)
$$

Then

$$
y_{n}(s)=\sum_{r=s}^{n-1} w_{s}(r) z(r) / b_{0}(s),
$$

where $b_{0}(s)$ is the $(s-i-j+1)$ st leading coefficient of $B^{i+j}$.

Remark: Equations (1.3.16), (1.3.17) are equivalent to the initial value problem

$$
\sum_{t=0}^{k} \hat{b}_{t}(r) w_{s}(r+t)=0, \quad r=s-k+1, s-k+2, \ldots
$$

with initial conditions $w_{s}(s-k+1)=\cdots=w_{s}(s-1)=0, w_{s}(s)=1$ : compare $(1.2 .1)-(1.2 .3)$ and $(1.2 .4 a)$.

Proof: Let $p=s-k+1, q=n, v=w_{s}$ and $u=y_{n}$ in (1.3.15). By virtue of (1.3.7), (1.3.16) and (1.3.17), the left side of (1.3.15) becomes

$$
\sum_{r=s}^{n-1} w_{S}(r) z(r)
$$

Since $u^{q, q+k-1}=y_{n}^{n, n+k-1}=0$, the second term on the right side of (1.3.15) is zero. Thus, expanding the right side and using (1.3.17), we derive 
$\left(v^{p, p+k-1}\right)^{\operatorname{Tr}}{ }_{B} p, p+k-I_{u} p, p+k-1$

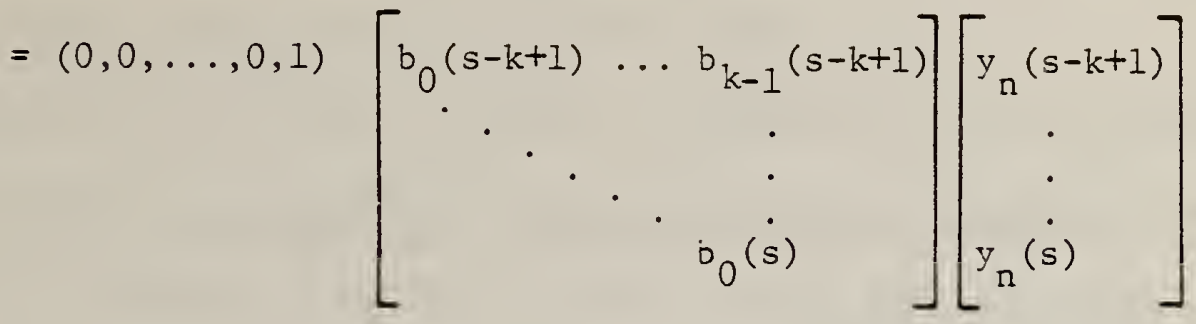

$$
\begin{aligned}
& =b_{0}(s) y_{n}(s) \text {. }
\end{aligned}
$$

Equating the left and right sides thus arrived at, we obtain (1.3.18).

In $\$ 3.2$ with appropriate conditions it will be proved that

$$
\lim _{n \rightarrow \infty} y_{n}(r)=y(r)
$$

for each fixed $r \geq i+j$. Thus, allowing $n \rightarrow \infty$ in (1.3.18), we have

$$
y(s)=\sum_{r=s}^{\infty} w_{s}(r) z(r) / b_{0}(s)
$$

for each $s \geq i+j+k-1$. Similarly, if

$$
\eta_{n}(s)=y(s)-y_{n}(s)
$$

is the truncation error incurred in accepting the approximation $\mathrm{y}_{\mathrm{n}}$ (s) for $y(s)$, we have

$$
\eta_{n}(s)=\sum_{r=n}^{\infty} w_{s}(r) z(r) / b_{0}(s)
$$

for each $s \geq i+j+k-1$.

Let us compare $(1.3 .20)$ with $(1.1 .2)$, the truncation error expansion for the original algorithm of Olver. Suppose $\ell=2$ and $j=k=1$. 
The difference operator which is implicit in $(1.1 .1)$ is $D=\left[d_{0}, d_{1}, d_{2}\right]$ winere

$$
d_{0}(r)=a(r+1), d_{1}(r)=-b(r+1), d_{2}(r)=c(r+1)
$$

for $r=0,1,2, \ldots$. Similarly, the right side of (1.1.1) is, in our present notation,

$$
g(r)=d(r+1), r=0,1,2, \ldots .
$$

The rows of the $(0,1)$-factorization $D_{1}^{0}=A_{1}^{0} B^{1}$ are scaled so that

$$
b_{0}(r)=p(r+1), b_{1}(r)=-p(r)
$$

for $r=1,2, \ldots$, where $B^{1}=\left[b_{0}^{1}, b_{1}^{1}\right]$ and $p=(p(0), p(1), \ldots)$ is the sequence defined in $\$ 1.1$. The solution $z^{1}$ of the forward elimination stage of the algorithm is given by

$$
z(r)=e(r), r=1,2, \ldots ;
$$

compare (1.3.8). Finally, it may be verified readily that the solution of the adjoint equation $\hat{B}_{w_{S}}(r)=0, r=s, s+1, \ldots$ such that $w_{s}(s)=1$ is given by

$$
w_{s}(r)=\frac{p(s) p(s+1)}{p(r) p(r+1)}, \quad r=s, s+1, \ldots .
$$

Aaking these substitutions into $(1.3 .20)$ and exchanging the roles of $r$ and $s$, we obtain (1.1.2).

The simple form of the equation for $w_{S}(r)$ depends solely on the particular scaling of the $(0,1)$-factorization used by olver and the fact that $k=1$. A similar simplification can be obtained for difference equations of arbitrary order provided only that $k=1$. It stems from 
the fact that the order of the adjoint equation is one, hence all solutions can be expressed as multiples of a single particular solution. These conclusions were also arrived at in Cash's paper [6].

The expansions (1.3.19) and (1.3.20) may be employed in the estimation of the optimal value of $n$ such that a given termination criterion is met. Typical termination criteria would be to require one or more values of $y_{n}(r)$ in the range $i+j \leq r \leq m$ to approximate $y(r)$ to a specified absolute or relative precision. Since the back substitition stage is stable, controlling the absolute or relative error only at the point $r=m$ is often sufficient. In practice equations (1.3.19) and (1.3.20) are employed by replacing the upper limit by $n+\nu$, where $\nu \geq 1$ is a parameter chosen to suit the problem at hand.

The solution of $(1.3 .16),(1.3 .17)$ is obtained by forward recurrence. The stability of this process will depend on the behavior of ${ }_{S}(r)$ for large $r$ relative to the other solutions of the adjoint equation. In general there is no reason to expect that $\omega_{S}(r)$ will not contain a component of a maximal solution. Thus the solution of (1.3.16) will normally be stable, although there could be an initial range where some unstable rounding error propagation takes place. 


\subsection{Constant-Coefficient Operators}

Let $D=\left[d_{0}, d_{1}, \ldots d_{l}\right] \leqslant D_{l}$ be a constant-coefficient operator. By this we mean that there exist $\delta_{0}, \delta_{1}, \ldots, \delta_{l} \in F$ such that $\delta_{0} \neq 0$, $\delta_{l} \neq 0$ and $d_{s}(r)=\delta_{s}$ for all $r$. We assume without loss of generality that $\delta_{l}=1$ in every constant-coefficient operator. Since every constant-coefficient operator is nonsingular, by our definitions, the kernel $K(D)$ has dimension $l$; see discussion preceding Definition 1.3.1.

A particular basis, which we shall call the standard basis, exists for each constant-coefficient operator; see [14,§\$13.0-13.1]. The polynomial

$$
P(\xi)=\xi^{l}+\delta_{l-1} \xi^{l-1}+\cdots+\delta_{0}
$$

is known as the characteristic polynomial for $D$. The standard basis is the set of sequences

$$
\therefore=\left\{\left(r^{m} \lambda^{r}\right) \in S \mid P(\lambda)=0 \text { and } m \in\{0,1, \ldots, \mu(\lambda)-1\}\right\}
$$

where $\mu(\lambda)$ denotes the multiplicity of the root $\lambda$. We are using the notation $\left(r^{m} \lambda^{r}\right)$ here as an alternative way of denoting the sequence

$$
\left(\rho_{m}, \lambda, 2^{m} \lambda^{2}, 3^{m} \lambda^{3}, \ldots\right)
$$

where $\rho_{0}=1$ and $P_{m}=0$ when $m>0$.

The standard basis of a constant-coefficient operator is not necessarily totally ranked; recall Definition 1.3.1. For example, if $\lambda_{1}$ and $\lambda_{2}$ are two distinct zeros of the characteristic polynomial such that $\left|\lambda_{1}\right|=\left|\lambda_{2}\right|$ then $\left|\lambda_{1}^{r}\right|=\left|\lambda_{2}^{r}\right|$ for all $r$. Neither solution dominates the other. 
Let us write $a<b$ whenever $a, b \in S$ are such that $a$ is dominated by $b$. We shall say that $a$ and $b$ are separated (at infinity) if $a<b$ or $b<a$. A totally ranked basis is linearly ordered by $<$ but in general the standard basis is not, as we have just seen. However, we can introduce a linearly ordered partition of the standard basis. We sha11 proceed to do this.

It is apparent that $\left(r^{m} \lambda_{1}^{r}\right)<\left(r^{m} \lambda_{2}^{r}\right)$ if, and only if, either $\left|\lambda_{1}\right|<\left|\lambda_{2}\right|$ or (ii) $\left|\lambda_{1}\right|=\left|\lambda_{2}\right|$ and $m_{1}<m_{2}$. Let $\sigma$ denote the number of roots of $P(\xi)=0$ of distinct absolute value, and let $\lambda_{1}, \lambda_{2}, \ldots, \lambda_{\sigma}$ be representative roots of $P(\xi)=0$ such that $\left|\lambda_{s}\right|<\left|\lambda_{s+1}\right|$ for each s. Define $\mu_{s}=\max \left\{\mu(\lambda)|| \lambda|=| \lambda_{s} \mid\right\}$. We define the sets

$$
\Lambda_{s}=\left\{\left(r^{m} \lambda^{r}\right) \in \Lambda|| \lambda|=| \lambda_{s} \mid\right\}, s=1,2, \ldots, \sigma
$$

and, for each $s$, the sets

$$
\Lambda_{s, m}=\left\{\left(r^{m} \lambda^{r}\right) \in \Lambda_{s} \mid \psi(\lambda) \geq m\right\} \quad, m=0,1, \ldots, \mu_{s}-1 .
$$

It may be verified readily that the sets $\Lambda_{s, m}$ are disjoint and nonempty and

$$
\Lambda=\bigcup_{s=1}^{\sigma} \stackrel{u}{\mathrm{~m}=0}_{u^{-1}}^{\mu_{\mathrm{s}}, \mathrm{m}}
$$

The sets $i_{\mathrm{s}, \mathrm{m}}$ are linearly ordered according to the definition: $\Lambda_{s, m}<\Lambda_{s^{\prime}, m^{\prime}}$ if, and only if, either (i) $s<s^{\prime}$ or (ii) $s=s^{\prime}$ and $\mathrm{m}<\mathrm{m}^{\prime}$.

An illustrative example is afforded by the constant-coefficient equation 


$$
\begin{gathered}
y(r+8)-(4+4 \sqrt{2}) y(r+7)+(15+16 \sqrt{2}) y(r+6)-(48+12 \sqrt{2}) y(r+5) \\
-12 y(r+4)+(192+64 \sqrt{2}) y(r+3)-(208+256 \sqrt{2}) y(r+2)+(256+192 \sqrt{2}) y(r+1) \\
-192 y(r)=0 .
\end{gathered}
$$

Its characteristic polynomial, in real factored form, is

$$
(\xi-1)(\xi-2)(\xi+2)\left(\xi^{2}-2 \sqrt{2} \xi+4\right)^{2}(\xi-3)=0 .
$$

The roots of (2.1.2) include the complex numbers $\sqrt{2}(1+i)$ and $\sqrt{2}(1-i)$, each of multiplicity two. The subsets of the linearly ordered partition in this example are

$$
\begin{aligned}
& \Lambda_{1,0}=\left\{1^{r}\right\} \\
& \Lambda_{2,0}=\left\{2^{r},(-2)^{r}, \sqrt{2}^{r}(1-i)^{r}, \sqrt{2}^{r}(1+i)^{r}\right\} \\
& \Lambda_{2,1}=\left\{r \sqrt{2}^{r}(1-i)^{r}, r \sqrt{2}^{r}(1+i)^{r}\right\} \\
& \Lambda_{3,0}=\left\{3^{r}\right\} .
\end{aligned}
$$

Note that if $a \in \Lambda_{2,0}$ and $b \in \Lambda_{2,1}$ then $|a(r) / b(r)|=r^{-1}$ when $r \geq 1$. In contrast, if $a \in \Lambda_{s}$ and $b \in \Lambda_{s}$, where $s<s^{\prime}$ then $|a(r) / b(r)|$ contains a decreasing exponential factor.

In general, if $a, b \in \Lambda$ are such that $a<b$, then the separation ratios $|a(r) / b(r)|$ decrease as the product of a power of $r^{-1}$ and an exponential function in $r$. If the exponential function is identically equal to one, as will be the case whenever $a$ and $b$ are standard basis solutions which correspond to characteristic roots of equal magnitude, then we shall say that $a$ and $b$ are algebraically separated. Otherwise, we shall say that $a$ and $b$ are exponentially separated.

Figure 5 is obtained by plotting the roots of (2.1.2) in the complex plane and drawing circles through them, centered at the origin. There are three circles, corresponding to the distinct absolute values of the roots. 
The double roots are indicated on the circle of radius two; it is helpful to imagine them duplicated on a second circle of radius two that is raised slightly out of the plane. Let us number the four circles, thus arrived at, by increasing three-dimensional distance from the origin.

This determines geometrically the proper linear ordering of the four subsets $\Lambda_{s, m}$ of the partition. The ordering of elements within any one of the subsets is unimportant for our purposes.

Clearly, this geometric indication of the linear ordering of the subsets of the partition can be extended to any constant-coefficient operator.

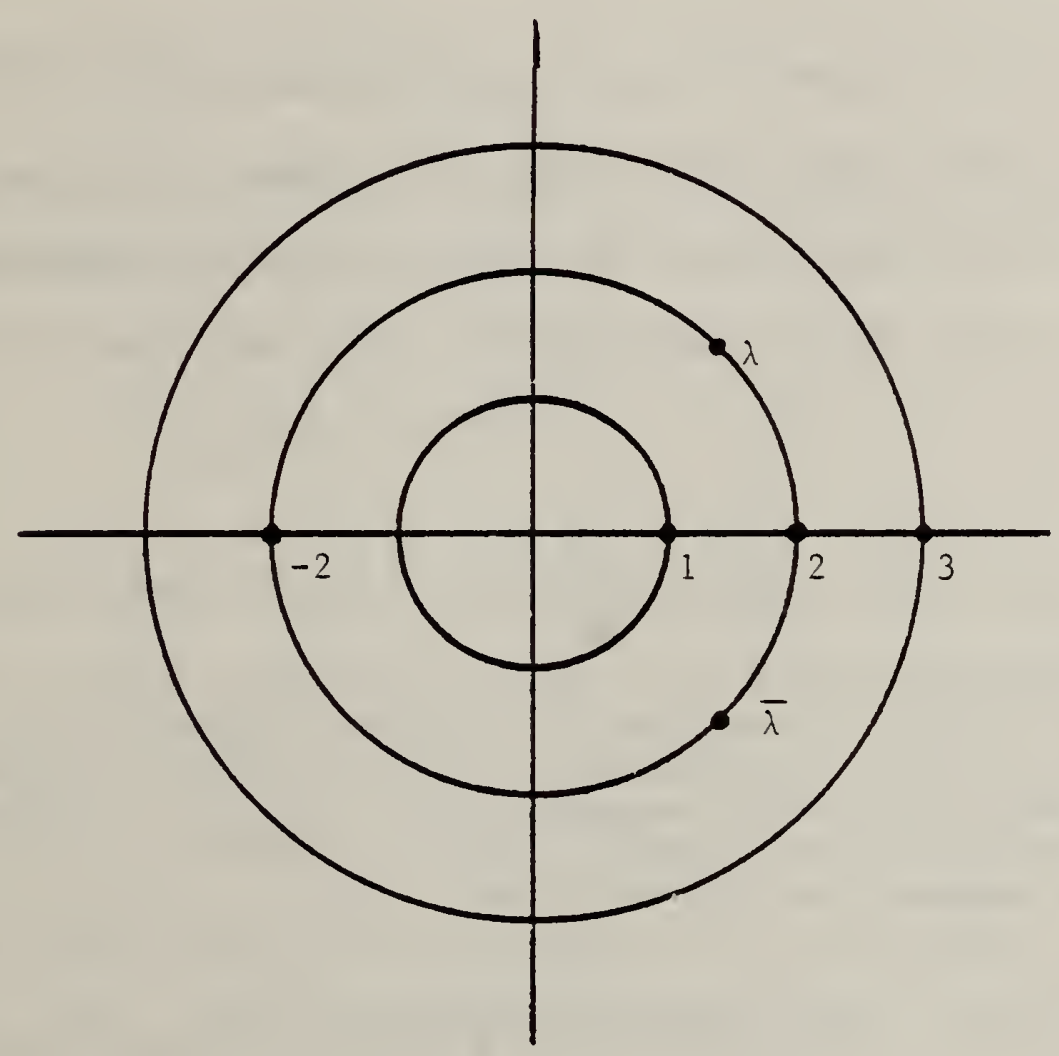

Figure 5. The roots of $(2.1 .2)$. The double roots $\sqrt{2}(1 \pm i)$ are denoted $\lambda, \bar{\lambda}$. 
Zhecrem 2.1.1 Let (2.1.1) denote the linearly ordered partition of the standard basis for a given constant-coeficient operator. Then:

i) If $a \leq i s, m$ and $b \leq s^{\prime}$, , , then $a<b$ if, and only if, $'_{s, z}={ }^{\prime} s^{\prime}, m^{\prime}$.

ii) If $a, b \leqslant \wedge_{s, m}$, then $|a(r)|=\mid b(r)$ for all $r$.

iii) If $a_{1}, a_{2}, \ldots, a_{q}, b=\wedge_{s, m}$ where the $a$ 's are distinct, and $z_{1}, z_{2}, \ldots, z_{q}=F$ are all nonzero, then

$$
\limsup _{r \rightarrow \infty} \sum_{p=1}^{q} \tilde{a}_{p} a_{p}(r) / b(r) \mid>0
$$

Proof: Parts (i) and (ii) are trivial consequences of our definitions. Iurning to part ( $i$ i $i$ ), we nota that the sequence $\sum_{p=1}^{q} a_{p} a_{p}(r) / b(r)$ is nor identically zero (since the a's are linearly independent). Let $\ddots_{1}, i_{2}, \ldots, \lambda_{q}, i$ be the characteristic roots associated with the set $\hat{s}_{s, m}$ such that

$$
a_{p}(r)=r^{a_{\lambda}} \prod_{p}, p=1,2, \ldots, q
$$

and $b(r)=z^{2} \lambda^{r}$. Since the $\lambda^{\prime}$ s lie on the same circle in the complex plane, there exist real constants $\hat{\gamma}_{1}, \hat{\gamma}_{2}, \ldots, \beta_{q}, \hat{F}$ all lying in the halfopen real interval $\left(\boldsymbol{\sim}^{-}, \sim\right)$ such that

$$
a_{p}(r)=r^{m}|\lambda|^{r} e^{i r \hat{p}_{p}}, \quad p=1,2, \ldots q
$$

and $b(r)=r^{m}, r^{r} \exp ($ ir $\beta), i=\sqrt{-1}$. Thus

$$
\sum_{p=1}^{q} a_{p} a_{p}(r) / b(r)=\sum_{p=1}^{q} \approx_{p} e^{i r / p}
$$

where $r_{p}=3_{p}-\beta_{r}$. The $r^{\prime} s$ are distinct real rumbers (modulo 2r) 
because the sequences $a_{1}, a_{2}, \ldots, a_{q}$ are assumed distinct.

The right member of the last equation represents a so-called almost periodic function of $r$ when $r$ is regarded as a continuous real variable. To complete the proof we appeal to the following lemma, the proof of which is given in the Appendix.

Lemma 2.1.1 Let $\alpha_{1}, \alpha_{2}, \ldots, \alpha_{q}$ be nonzero complex numbers and $\gamma_{1}, \gamma_{2}, \ldots$, $\gamma_{q}$ distinct real numbers (modulo $2 \pi$ ). Define the infinite sequence

$(2.1 .4) \quad x(r)=\sum_{p=1}^{q} \alpha_{p} e^{i r \gamma_{p}} \quad, \quad r=0,1,2, \ldots$.

Then $\lim \sup _{r \rightarrow \infty}|x(r)|>0$.

An equivalent statement to inequality (2.1.3) is that there exists an infinite subsequence of the sequence

$$
\sum_{p=1}^{q} a_{p} a_{p}(r) / b(r), \quad r=0,1,2, \ldots
$$

which is bounded away from zero. Intuitively, this means that it is impossible to create a new sequence with qualitatively different asymptotic behavior merely by forming a Iinear combination of standard basis solutions of similar asymptotic behavior. Note, however, that unlike the original sequences infinitely many zeros may occur in the new sequence. For example, if $2^{r}$ and $(-2)^{r}$ are sequences in a standard basis, then every other term of the sequence $2^{r}+(-2)^{r}$ is zero.

\footnotetext{
We relegate the proof of Lema 2.1.1 to the Appendix because the theory of almost periodic functions is not germane to the rest of this thesis.
} 


\subsection{Separable Operators}

In this section we introduce a subclass of the general set of infinite upper-triangular band matrices $D_{\ell}$ introduced in $\$ 1.2$, and examine in detail the general structure of the kernel of an operator in this subclass. This will prepare the way for the presentation, in the next section, of a general classification of all sequences in $S$ with respect to a given linear difference operator. In Theorem 2.1.1 we presented three properties of the standard basis of a constant-coefficient operator. These properties suggest the following extension of Definition 1.3.1: Definition 2.2.1 Let $D \in D_{l}$ be such that $K(D)$ has dimension $l$. Then D will be said to be separable if there exists a basis X for D which can be partitioned into nonempty disjoint subsets $\mathrm{x}_{1}, \mathrm{x}_{2}, \ldots, \mathrm{x}_{\sigma}$ in such a way that the following three conditions are all satisfied:

i) If $x \in x_{s}$ and $y \in x_{t}$, where $s<t$, then $\lim _{r \rightarrow \infty} x(r) / y(r)=0$;

ii) If $x, y \in x_{s}$ for some $s$, then $0<\lim$ inf $r_{r \rightarrow \infty}|x(r) / y(r)|$ and $\lim \sup _{r \rightarrow \infty}|x(r) / y(r)|<\infty$;

iii) If $x$ is any linear combination of sequences from $x_{s}$, other than $x=0$, and $y \in x_{s}$, then $\lim \sup _{r \rightarrow \infty}|x(r) / y(r)|>0$.

Let $D$ be a separable operator. A basis $X$ for $D$ which is such that

$$
X=\bigcup_{s=1}^{\sigma} x_{s},
$$

where the ${ } \mathrm{s}$ satisfy the conditions of Definition 2.2 .1 will be said to be oprimally ranked. Let

$$
x_{s}=\left\{x_{s, 1}, x_{s, 2}, \ldots, x_{s, n_{s}}\right\}
$$

where the subscript $n_{S}$ is positive (because $x_{S}$ is nonempty). 
Obviously, we have

$$
l=\sum_{s=1}^{\sigma} n_{s} .
$$

In Lemna 2.2.1 below we will show that two distinct optimally ranked bases for a given separable operator have the same number $(\sigma)$ of disjoint subsets and the same number $\left(n_{s}\right)$ of sequences in corresponding subsets.

An optimally ranked basis exhibits the full range of possible asymptotic behavior of solutions to the corresponding homogeneous difference equation. Conditions (i) and (ii) of Definition 2.2.1 establish the ranking of sequences within the basis. If (2.2.1) is a particular optimally ranked basis, we shall say that $x \in X$ is a sequence of type $s$ provided that $x \in X_{s}$. More generally, we shall say that $x \in K(D)$ is a sequence of type $\underline{s}$ provided that $x \neq 0$ and there exist scalars $a_{p, q} \in F$ such that

$$
x=\sum_{p=1}^{s} \sum_{q=1}^{n} \alpha_{p, q} x_{p, q},
$$

where $a_{s, q} \neq 0$ for some $q, 1 \leq q \leq n_{s}$. Such a representation of $x$ exists and is unique for every nonzero $x \in K(D)$. Again, in consequence of Lemma 2.2.1 below, if we change to a different optimally ranked basis, the type of every sequence in $K(D)$ remains invariant. Condition (iii) of Definition 2.2.1 presages this; it requires that no nontrivial linear combination of basis sequences of the same type may be dominated by a basis sequence of that type.

Let $L(I)$ denote the linear subspace of $S$ which is spanned by $I$ where $I \subset S$ may be any finite subset of sequences. If (2.2.1) is an optimally ranked basis for a separable operator $D$, then we shall say that $s$ the subspace $L\left(U x_{p}\right)$ is the subdominant subspace of type s for $\underline{D}$. $\mathrm{p}=\mathrm{i}$ 
These subspaces are independent of the basis; again, see Lemma 2.2.1 below. If $j_{s}$ denotes the dimension of $L\left(\underset{p=1}{S} x_{p}\right)$, then

$$
j_{s}=\sum_{p=1}^{s} n_{p}
$$

compare (2.2.2) and (2.2.3). In addition, we shall say that $\{0\}$, the linear subspace of dimension zero (whose only sequence is the zero sequence) is the subdominant subspace of type 0 .

Let $U$ be one of the subdominant subspaces for $D$. The complementary subspace of $U$ in $K(D)$, i.e., the subspace $V$ of $K(D)$ such that

$$
K(D)=U \oplus V \text {, }
$$

will be called the corresponding dominant subspace. The symbol $\oplus$ on the right side of $(2.2 .6)$ means that $K(D)$ is the direct sum of the subspaces $U$ and $V$; that is, every nonzero sequence $x \in K(D)$ is uniquely expressible in one of the forms $x=u$, or $x=v$, or $x=u+v$, where $u \in U$ and $v \in V$. Clearly, if $U$ is the subdominant subspace of type $s, 0 \leq s<\sigma$, then $V=L\left(\underset{p=s+1}{\|} \mathrm{x}_{\mathrm{p}}\right)$; and if $U$ is the subdominant subspace of type $\sigma$, then $V=\{0\}$.

Before stating and proving Lemma 2.2.1, we note that

$$
L\left(\bigcup_{p=1}^{S} X_{p}\right)=L\left(X_{1}\right) \oplus L\left(X_{2}\right) \oplus \cdots \oplus L\left(X_{s}\right)
$$

Thus, an alternative characterization of a sequence of type $s$ in $K(D)$ is the following: $x \in K(D)$ is of type $s, l \leq s \leq \sigma$, provided that

$$
x=\sum_{p=1}^{s} x_{p}, x_{p} \in L\left(x_{p}\right), \quad x_{s} \neq 0
$$


Lemma 2.2.1 Let

$$
X=\bigcup_{s=1}^{\sigma} X_{s}=\bigcup_{s=1}^{\sigma}\left\{x_{s, 1}, x_{s, 2}, \ldots, x_{s, m_{s}}\right\}
$$

and

$$
Y=\bigcup_{s=1}^{\tau} Y_{s}=\bigcup_{s=1}^{\tau}\left\{y_{s, 1}, y_{s, 2}, \ldots, y_{s, n_{s}}\right\}
$$

be distinct optimally ranked bases for a separable operator $D \leqslant D_{l}$.

Then $\sigma=\tau$ and $\mathrm{m}_{\mathrm{s}}=\mathrm{n}_{\mathrm{s}}$ for all $\mathrm{s}$. Furthermore,

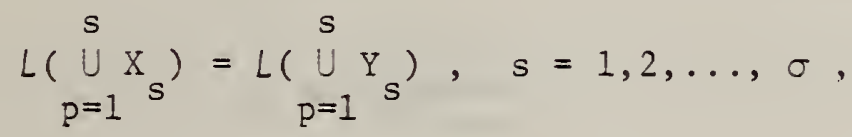

and the type of every nonzero sequence $z \in K(D)$ is the same with respect to $\mathrm{X}$ as it is with respect to $\mathrm{Y}$.

Proof Let $u \in X_{S}$ and $v \in X_{t}$, so that relative to $X$ the types of $u$ and $v$ are $s$ and $t$. Let the types of $u$ and $v$ relative to $Y$ be $s^{\prime}$ and $t^{\prime}$. Then there exist unique representations

$$
u=\sum_{j=1}^{s^{\prime}} u_{j}, u_{j} \in L\left(Y_{j}\right), u_{s}, \neq 0
$$

and

$$
v=\sum_{j=1}^{t^{\prime}} v_{j}, \quad v_{j} \in L\left(Y_{j}\right), v_{t^{\prime}} \neq 0 .
$$

Furthermore, by condition (i) of Definition 2.2.1, we have, as $r \rightarrow \infty$,

$$
u(r)=u_{s^{\prime}}(r)+o\{y(r)\}, y \in Y_{s^{\prime}}
$$




$$
v(r)=v_{t^{\prime}}(r)+o\{y(r)\}, y \in Y_{t^{\prime}} .
$$

i) First we prove that $s^{\prime}=t^{\prime}$ if $s=t$, i.e., if $u$ and $v$ are of the same type with respect to $x$, then they are also of the same type with respect to $Y$.

Since $s=t$, the sequence $u(r) / v(r)$ is asymptotically bounded away from zero; compare part (ii) of Definition 2.2.1. Suppose that $s^{\prime}<t^{\prime}$. We shall obtain a contradiction by showing that, under this assumption, $u(r) / v(r)$ has an infinite subsequence which converges to zero. Choose any $y \in Y_{t^{\prime}}$. Then we have

$$
\lim _{r \rightarrow \infty} \frac{u(r)}{y(r)}=0
$$

Erom (2.2.8), we have

$$
\frac{v(r)}{y(r)}=\frac{v_{t^{\prime}}(r)}{y(r)}+o(1)
$$

and, using the triangle inequality, an elementary property of the limit superior, and condition (iii) of Definition 2.2.1, we obtain

$$
\limsup _{r \rightarrow \infty}\left|\frac{v(r)}{y(r)}\right|=\underset{r \rightarrow \infty}{\lim \sup }\left|\frac{v_{t^{\prime}}(r)}{y(r)}\right|>0 .
$$

Thus there exists an infinite subsequence of $v(r) / y(r)$ that is bounded away from zero, and therefore also a subsequence of

$$
\frac{u(r)}{v(r)}=\frac{u(r) / y(r)}{v(r) / y(r)}
$$

that converges to zero. This supplies the needed contradiction. 
A similar contradiction is obtained if we suppose that $s^{\prime}>t^{\prime}$. Hence $s^{\prime}=t^{\prime}$.

ii) Next we prove that $s^{\prime}<t^{\prime}$ if $s<t$.

Since $s<t$, the sequence $u(r) / v(r)$ converges to zero. Suppose that $s^{\prime} \geq t^{\prime}$. We shall derive a contradiction by showing that, under this assumption, $u(r) / v(r)$ has a subsequence which does not converge to zero. Choose any $y \in Y_{S^{\prime}}$. First, by using $(2.2 .7)$ and an argument similar to that used in part (i) of this proof, we have

$$
\lim _{r \rightarrow \infty} \sup \left|\frac{u(r)}{y(r)}\right|>0 .
$$

Thus there exists an infinite subsequence of $u(r) / y(r)$ that is bounded away from zero.

Assume now that $s^{\prime}>t^{\prime}$. Then

$$
\lim _{r \rightarrow \infty} \frac{v(r)}{y(r)}=0
$$

In this case it follows that there exists a subsequence of

$$
\frac{u(r)}{v(r)}=\frac{u(r) / y(r)}{v(r) / y(r)}
$$

that diverges to $\infty$, which supplies the needed contradiction.

Alternatively, assume that $s^{\prime}=t^{\prime}$. Using (2.2.8), we have for sufficiently large $r$

$$
\left|\frac{v(r)}{y(r)}\right| \leq\left|\frac{v_{t^{\prime}}(r)}{y(r)}\right|+1 .
$$

Furthermore, using condition (ii) of Definition 2.2.1, we see that there exist $r_{0} \geq 0$ and $\beta>0$ such that 


$$
\left|\frac{v^{\prime}(r)}{y(r)}\right| \leq \hat{r}, \quad r \geq r_{0} .
$$

Inerefore, for sufficiently large $r$, we have

$$
\left|\frac{\mathrm{v}(r)}{\mathrm{y}(r)}\right| \leq \beta+1
$$

and

$$
\frac{u(r)}{V(r)}\left|\geq(1+\hat{p})^{-1}\right| \frac{u(r)}{y(r)} \mid .
$$

It jollows that $u(r) / v(r)$ has a subsequence that is bounded away from zerc, which again supplies the needed contradiction.

iii) Vext, we prove that $\sigma=\tau$ and $m_{s}=n_{s}$ for all $s$, and also that $L\left(\bigcup_{p=1}^{S} X_{S}\right)=L\left(\bigcup_{p=1}^{S} Y_{S}\right)$ for all $s$.

Part (i) of this proof implies that for each $s, 1 \leq s \leq \sigma$, the type relative to $Y$ of every sequence in $X_{S}$ is the same, say $t_{S}$. Using part (ii), we have$$
1 \leq t_{1}<t_{2}<\cdots<t_{\sigma} \leq \tau \text {. }
$$

Furthermore, that $s \leq t_{s}$ for all $s, l \leq s \leq \sigma$, is easily proved by induction. In particular, $\sigma \leq t_{\sigma}$ and, using (2.2.9), we see that $\sigma \leq \tau$. Inverting the roles of $X$ and $Y$ and repeating the argument, we see that $\tau \leq \tau$. Thus $\tau=\tau$, and $(2.2 .9)$ implies $t_{s}=s$ for all $s$. For each $s$, it follows that

$$
L\left(X_{1}, \ldots \mid j X_{s}\right) \subset L\left(Y_{1}, \cdots \cup Y_{s}\right)
$$




$$
L\left(Y_{1} U \cdot \cdots Y_{s}\right) \subset L\left(X_{1} U \cdot U X_{s}\right)
$$

Thus

$$
L\left(X_{1} \cup \cdots \cup X_{s}\right)=L\left(Y_{1} U \cdot \cup Y_{s}\right)
$$

for each $s$. In particular, $L\left(X_{1}\right)=L\left(Y_{1}\right)$. Since $X_{1}$ and $Y_{1}$ are bases of the same linear space, we have $\mathrm{m}_{1}=\mathrm{n}_{1}$. An easy induction completes the proot of this part.

iv) To complete the procf, let $z \in K(D)$ be a nonzero sequence such that

$$
z=\sum_{p=1}^{s} u_{p}, u_{s} \neq 0, u_{p} \in L\left(X_{p}\right)
$$

and

$$
z=\sum_{p=1}^{t} v_{p}, \quad v_{t} \neq 0, v_{p} \in L\left(Y_{p}\right)
$$

Assume $s<t$, and let $y \in Y_{t}$. Then

$$
\lim _{r \rightarrow \infty} \frac{v_{t}(r)}{y(r)}=\lim _{r \rightarrow \infty}\left[\sum_{p=1}^{s} \frac{u_{p}(r)-v_{p}(r)}{y(r)}-\sum_{p=s+1}^{t-1} \frac{v_{p}(r)}{y(r)}\right]=0 .
$$

But this result contradicts condition (iii) of Definition 2.2.1. Hence we must have $s \geq t$. A similar contradiction is obtained if we assume $s>t$. Therefore $s=t$ and the proot of Lemma 2.2.I is complete.

In general, the actual determination of optimally ranked bases for variable coefficient difference operators is a difficult problem. Some results have been given by Wimp [26]. This reference extends earlier work by Birkhoff and Trjitzinsky [2,3] on the analytic theory of singular difference equations whose coefficients possess asymptotic expansions 
of a prescribed form. All equations with coefficients rational in r are included, for example. Wimp's analysis provides a =eans of constructing a basis, which he calls a canonical set: see [26, Theorem 3.3 and Definition 3.6]. The canonical set is analogous to our optimally ranked basis. More specifically, there exists a basis $\left\{y_{1}, y_{2}, \ldots, y_{l}\right\}$ such that, for each $s=1,2, \ldots, l$,

$$
y_{s}(r)=c_{s} M_{s}(r)\{1+o(1)\} \quad \text { as } r \rightarrow \infty
$$

where $c_{s} \neq 0$ and

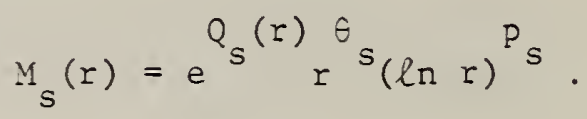

Here $\epsilon_{S}$ is a complex number and $P_{S}$ is a positive integer. $Q_{S}$ is of the form

$$
Q_{S}(r)=\mu_{0} r \ln r+\mu_{1} r+u_{2} r^{I-1 / \rho}+\cdots+\mu_{0} r^{1 / \rho}
$$

where $\rho$ is an inreger, $\rho \geq 1$, and $\mu_{0}, \mu_{1}, \ldots, \mu_{\rho}$ are complex numbers. The rich variety of possible separation ratios of solutions as $r \rightarrow \infty$, compared with the algebraic or exponential separation of solutions in the constant-coefficient case, described in 2.1 above, is evident. 


\subsection{The General Classification}

In $\$ 2.2$ we showed that every nonzero sequence in the kernel of a separable operator has a unique type with respect to that particular operator. In this section we extend this classification to every nonzero sequence in $S$. The following definition should be compared with Definition 1.3 .2 .

Definition 2.3.1 Let $D \in D_{l}$ be separable with optimally ranked basis $\mathrm{X}=\mathrm{U}_{\mathrm{S}=1} \mathrm{X}_{\mathrm{S}}$. Then, for each $\mathrm{y} \in S$, we say that type $(\mathrm{y})=s$, or more fully, the type of $y$ with respect to $\underline{D}$ is $s$, provided that $0<s<c$ and the following two conditions are satisfied:

i)

$$
\text { If } x \in x_{S+1} \text { then } \lim _{r \rightarrow \infty} \frac{y(r)}{x(r)}=0 \text {; }
$$

$$
\text { If } x \leqslant x_{s} \text { then } \infty \geq \underset{r \rightarrow \infty}{\lim \sup }\left|\frac{\mathrm{v}(r)}{\mathrm{x}(r)}\right|>0 \text {. }
$$

If ( $i$ ) is satisfied for $s=0$, then ( $i i)$ does not apply, and we define type $(y)=0$. Similarly, if (ii) is satisfied for $s=\sigma$, (i) does not apply, and we define type $(y)=\sigma$.

The classification of sequences introduced in Definition 2.3 .1 allows us to treat both homogeneous and inhomogeneous difference equations in a uniform manner when the difference operator is separable. The kernel of the operator contains every solution of the homogeneous equation, by definition. But solutions of the inhomogeneous equation with arbitary right-hand side may lie anywhere in $S$. This is why a classification of all sequences in $S$ is desirable.

Theorem 2.3.1 Every sequence in $S$ possesses an unambiguous type with respect to each separable operator in $\mathrm{O}_{2}$.

Proof (i) Eirst we show that the type with respect to a fixed optimally 
ranked basis is determined uniquely by Definition 2.3.1 for every nonzero sequence in $S$.

Let $z \in S$. Assuming that

$$
\lim _{r \rightarrow \infty} \sup \left|\frac{z(r)}{x(r)}\right|>0
$$

for at least one sequence $\mathrm{x} \in \mathrm{X}$, let $\hat{\mathrm{x}} \in \mathrm{X}$ be a sequence of largest type such that $(2.3 .1)$ is satisfied. Let $s=$ type $(\hat{x})$. Then $(2.3 .1)$ is satisfied for every $x \in X_{s}$, since

$$
\left|\frac{z(r)}{x(r)}\right|=\left|\frac{z(r)}{\hat{x}(r)}\right|\left|\frac{\hat{x}(r)}{x(r)}\right|
$$

and $\lim \inf _{r \rightarrow \infty}|\hat{x}(r) / x(r)|>0$ as a consequence of condition (ii) of Definition 2.2.1.

If $s=\sigma$, then type $(z)=\sigma$ according to Definition 2.3.1. Therefore suppose that $s<\sigma$. By the maximality of $\hat{x}$ we see that $\lim \sup _{r \rightarrow \infty}|z(r) / x(r)|=0$ whenever type $(x)>s$ or, equivalently,

$$
\lim _{r \rightarrow \infty} \frac{z(r)}{x(r)}=0 \text { whenever type }(x)>s
$$

Accordingly, type $(z)=s$.

To conclude the proof of part (i), let us suppose that (2.3.1) is not satisfied for any $x \in X$. Then (2.3.2) is satisfied for every $x \in X$, and in particular for every $x \in X_{I}$. Then type $(z)=0$.

(ii) In this part we prove that if $X=\bigcup_{s=1}^{\sigma} X_{s}$ and $X^{\prime}=\bigcup_{s=1}^{\sigma} X_{s}^{\prime}$ are two distinct optimally ranked bases for $D$ then the type of every sequence in $S$ is the same with respect to $X$ and $X^{\prime}$.

Choose any $s, 1 \leq s \leq \sigma$, and choose $x \in X_{s}, x^{\prime} \in X_{s}^{\prime}$. There 
exist $x_{p} \in x_{p}, 1 \leq p \leq s$, such that $x^{\prime}=x_{1}+\cdots+x_{s} ;$ see Lemma 2.2.1. Furthermore, we have

$$
\frac{x^{\prime}(r)}{x(r)}=o(1)+\frac{x_{S}(r)}{x(r)} \text { as } r \rightarrow \infty .
$$

Since $x_{s}$ is a linear combination of sequences of type $s$, a brief computation and application of condition (ii) of Definition 2.2.1 yields

$$
\lim _{r \rightarrow \infty}\left|\frac{x_{s}(r)}{x(r)}\right|<\infty .
$$

It follows that

$$
\begin{aligned}
& \underset{r \rightarrow \infty}{\lim \sup }\left|\frac{x^{\prime}(r)}{x(r)}\right|<\infty \\
& \text { and } \underset{r \rightarrow \infty}{\lim \inf }\left|\frac{x(r)}{x^{\prime}(r)}\right|>0 \text { whenever type }\left(x^{\prime}\right)=\text { type }(x) .
\end{aligned}
$$

Since the roles of $x$ and $x^{\prime}$ are interchangeable, we also have

$(2.3 .4) \quad \lim _{r \rightarrow \infty}\left|\frac{x(r)}{x^{\prime}(r)}\right|<\infty$

$$
\text { and } \underset{r \rightarrow \infty}{\lim \inf }\left|\frac{x^{\prime}(r)}{x(r)}\right|>0 \text { whenever type }\left(x^{\prime}\right)=\text { type }(x) \text {. }
$$

Let $z \in S$ and suppose type $(z)=0$ with respect to $X$. Thus $z(r) / x(r) \rightarrow 0$ for every $x \in X$. Choose $x^{\prime} \in X^{\prime}$. Let $s=$ type $\left(x^{\prime}\right)$ and choose $\mathrm{x} \in \mathrm{x}_{\mathrm{S}}$. Then

$$
\left|\frac{z(r)}{x^{\prime}(r)}\right|=\left|\frac{z(r)}{x(r)}\right|\left|\frac{x(r)}{x^{\prime}(r)}\right| \rightarrow 0 \text { as } r \rightarrow \infty
$$

by virtue of $(2.3 .4)$. This proves type $(z)=0$ with respect to $X^{\prime}$. 
Let $z \leqslant S$ and suppose type $(z)=\sigma$ with respect to $X$. Thus $\lim \sup _{r \rightarrow \infty}|z(r) / x(r)|>0$ for every $x \in X$. Choose $x^{\prime} \div X^{\prime}$. Let $s=$ type $\left(x^{\prime}\right)$ and choose $x \in x_{s}$. Then the sequence

$$
\left|\frac{z(r)}{x^{\prime}(r)}\right|=\left|\frac{z(r)}{x(r)}\right|\left|\frac{x(r)}{x^{\prime}(r)}\right|
$$

satisiies $\lim \sup _{r \rightarrow \infty}\left|z(r) / x^{\prime}(r)\right|>0$ by virtue of (2.3.3). This proves type $(z)=\sigma$ with respect to $X^{\prime}$.

Finally, let $z \in S$ and suppose type $(z)=s, 0<s<\sigma$. Thus $z(r) / x(r) \rightarrow 0$ for every $x \in X_{s+1} U \cdot \cdots X_{\sigma}$ and $\lim \sup _{r \rightarrow \infty}|z(r) / x(r)|>0$ for every $x \in x_{S}$. It can be verified that $z(r) / x^{\prime}(r) \rightarrow 0$ for every $x^{\prime} \leqslant x_{s+1}^{\prime} \cdot \cdots X_{\sigma}^{\prime}$ and that $\lim \sup _{r \rightarrow \infty}\left|z(r) / x^{\prime}(r)\right|>0$ for every $x^{\prime} \leqslant x_{s}^{\prime}$ using arguments similar to the ones used in the preceding two paragraphs. 


\subsection{Preliminaries and Overview}

In this chapter we fix our attention on a particular linear difference equation

$$
\operatorname{Dy}(r)=g(r), \quad r=0,1,2 \ldots
$$

assuming that $D \leqslant D_{2}$ is separable and $g \in S$. More specifically, we are interested in computing an approximation $y_{n}$ of a particular solution $y$ of (3.0.1) which is valid over some finite subsequence $y^{i, m} . i$ and m given.

Let $\sigma$ be the number of distinct types in any optimally ranked basis for $D$. We assume that $y$ is known to be a sequence of type $t$ with respect to $D$, where $0 \leq t \leq \sigma$. We also assume that initial values $y(i), y(i+1), \ldots, y(i+j-1)$ are known, where $j$ is the dimension of the subdominant subspace of type $t$ for $D$. The extension of Theorem 1.3.1, from totally separable to separable operators, is given in $\$ 3.1$. Accordingly, these initial values (in the presence of one additional condition) suffice to determine the solution uniquely.

Let $k=2-j$. The approximating sequence $y_{n}$ is defined as the solution of (3.0.1) which satisfies the conditions

$$
y_{n}(i+r)=y(i+r), \quad r=0,1, \ldots, j-1
$$

and

$$
y_{n}(n+r)=0 \quad, \quad r=0,1, \ldots, k-1
$$

Since $y^{i, m}$ is to be approximated by $y_{n}^{i, m}$, it is clear that the value 
of $n$ must exceed $m$. The existence and uniqueness of the approximating sequences $y_{n}$, for all sufficiently large $n$, and the convergence of the sequence of approximate values $y_{n}(r)$ to $y(r)$ for each value of $r$ in the range $i+j \leq r<\infty$, are proved under appropriate conditions in $\$ 3.2$.

The general algorithm, which is valid for any separable operator, reduces to the algorithm presented in $\$ 1.3$ for totally separable operators. We again assume that the separable operator is ( $i, j$ ) - factorizable, just as we did for totally separable operators. The linear system $(1.3 .5)$ is solved by the factorization method by means of a forward elimination stage followed by a back substitution stage; see $\$ 1.3$. The expansions (1.3.19) and (1.3.20), which are used to estimate the optimal value of $n$, are unchanged for separable operators. In $\$ 3.3$ we discuss the stability of the forward elimination stage, which was omitted in $\$ 1.3$. We also extend Theorem 1.3.2 to separable operators, showing that the back substitution is stable. Finally, we note that our remarks on the stability of the forward recurrence solution of the adjoint equation, whose solution enters in the truncation error expansion of $\$ 1.3$, apply to separable operators as well as totally separable operators.

We conclude this introductory section by fixing some notation for later use in this chapter. Let

$(3 \cdot 0.4)$

$$
X=\bigcup_{s=1}^{\sigma} x_{s}
$$

be an optimally ranked basis for $D$, where

$$
x_{s}=\left\{x_{s, 1}, x_{s, 2}, \ldots, x_{s, n}\right\}, \quad s=1,2, \ldots, \sigma .
$$

Then the dimensinn of the subdominant subspace of type $t$ for $D$ is 
$(3.0 .6)$

$$
j=\sum_{s=1}^{t} n_{s} .
$$

Define the set

$(3.0 .7)$

$$
U=\left\{u_{1}, u_{2}, \ldots, u_{j}\right\}=U_{s=1}^{t} x_{s}
$$

by means of the correspondence

$$
x_{p, q}=u_{n_{1}}+n_{2}+\cdots+n_{p-1}+q
$$

When $t=0$ it is understood that $j=0$ and $U$ is empty. Similarly, define the set

$(3.0 .8)$

$$
V=\left\{v_{1}, v_{2}, \ldots v_{k}\right\}=\underset{s=t+1}{U} x_{s}:
$$

when $t=\sigma$ it is understood that $V$ is empty. 


\subsection{Existence and Uniqueness}

Let $y$ be a solution of $(3.0 .1)$ of type $t$ such that

$$
y(i+r)=r_{r}, \quad r=0.1, \ldots j-1
$$

for some $i \geq 0$, where $j$ is given by (3.0.6). Using the notation $|u|(i)$ for the Casoratian of $U$ at $i$, we also suppose that

$$
|\mathrm{U}|(i) \neq 0:
$$

see $(3.0 .7)$. Note that for every $i(3.1 .2)$ is satisfied vacuously if $t=0$, and also (by virtue of Cásorati's theorem $[14, \$ 12.11]$ ) if $t=\sigma$ and $D$ is nonsingular. The following theorem generalizes Theorem 1.3.1. Theorem 3.1.1 If (3.1.2) is valid then there exists a unique solution of (3.0.1) of type $t$ such that (3.1.1) is satisfied.

Eroof: Suppose $t=0$. Then $j=0$ and we must prove that without any specified initial values $\mathrm{y}$ is uniquely determined. Let $\mathrm{v} \in S$ be a sclution of $(3.0 .1)$, other than $y$, such that type $(v)=0$. Since $y-y \in K(D)$, there exist scalars $a_{p, q} \in F$ such that

$$
y-v=\sum_{p=1}^{\sigma} \sum_{q=1}^{n} a_{p, q} x_{p, q} ;
$$

see (3.0.5). At least one of the $\alpha_{p, q}$ is nonzero; let the largest value of $p$ such that $a_{p, q} \neq 0$ for some $q, 1 \leq q \leq n_{p}$, be $p=s$. Then

$$
\lim _{r \rightarrow \infty} \frac{y(r)-v(r)}{x_{s, 1}(r)}=0
$$

because type $(y)=$ type $(v)=0$. By part (i) of Definition 2.2.1, we 
have

$$
\lim _{r \rightarrow \infty} \sum_{p=1}^{s-1} \sum_{q=1}^{n p} \frac{\alpha_{p, q^{x} p, q}(r)}{x_{s, 1}(r)}=0 .
$$

It follows from the above three equations that

$$
\lim _{r \rightarrow \infty} \sum_{q=1}^{n} \frac{a_{s, q^{x}, q}(r)}{x_{s, I}(r)}=0 .
$$

But this result contradicts part (iii) of Definition 2.2.1; therefore we conclude that $y=v$.

Alternatively suppose $t>0$. Let $v \in S$ be a sequence of type $t$ which satisfies $(3.0 .1)$ and is coincident with $y(i+r)$ for $r=0,1, \ldots$, $j-1$. Then there exist scalars $a_{p, q} \in F$ such that

$$
v=y+\sum_{p=1}^{\sigma} \sum_{q=1}^{n} \alpha_{p, q}{ }^{x} p, q .
$$

Let the largest value of $p$ in this sum such that at least one of the scalars $a_{p, q}$ is nonzero be $p=s$. Then $s \leq t$, because $s>t$ would inply

$$
\lim _{r \rightarrow \infty} \frac{v(r)-y(r)}{x_{s, 1}(r)}=0
$$

(since type $(v)=$ type $(y)=t$ ) and

$$
\lim _{r \rightarrow \infty} \sum_{p=1}^{s-1} \sum_{q=1}^{n p} \frac{\alpha_{p, q^{x}, q^{(r)}}}{x_{s, 1}(r)}=0
$$

(by part (i) ô Definition 2.2.1). This gives 


$$
\lim _{r \rightarrow \infty} \sum_{q=1}^{n} \frac{a_{s, q^{x} s, q}(r)}{x_{s, 1}(r)}=0
$$

which is a contradiction.

Thus, $a_{p, q}=0$ whenever $p>s$. The $a_{p, q}$ having $p \leq s$ satisfy the system of linear equations

$$
\sum_{p=1}^{s} \sum_{q=1}^{n} a_{p, q} x_{p, q}(i+r)=0, \quad r=0,1, \ldots, j-1 .
$$

This system is nonsingular by the condition $|U|(i) \neq 0$, so we have $a_{p, q}=0$ for all $p$ and $q$. Thus $v(r)=y(r)$ for all $r$ and the theorem is proved.

Theorem 3.1.2 If $D$ is nonsingular then, for each $i \geq 0,|U|(i+r) \neq 0$ for at least one value of $r$ in the range $0 \leq r \leq ?-j$.

Proof: Since $D$ is nonsingular, Casorati's theorem is valid, i.e., $x i(i) \neq 0$ for all $i$. Theorem 3.1 .2 is then an immediate consequence of Laplace's general theorem on the expansion of determinants $[15, \$ 93]$. Theorem 3.1.2 implies there is no shortage of points $i$ which satisfy condition (3.1.2) when the difference operator is nonsingular. Our next goal is to show that any optimally ranked basis may be used to locate an admissible value of i.

Theorem 3.1 .3 If $|U|(i) \neq 0$ for some fixed $i \geq 0$, then the leading principal minor of order $j$ of the Casoratian evaluated at $i$ of every optimally ranked basis for $D$ is nonzero.

Proof: If $j=0$ then there is nothing to prove, so let us assume $j>0$. Also, let us assume temporarily that $j<\ell$. Let $X^{\prime}$ be an optimally ranked basis distinct from $X$, where

$$
X^{\prime}=\bigcup_{S=1}^{\sigma} X_{s}^{\prime}
$$


and

$$
X_{s}^{\prime}=\left\{x_{s, 1}^{\prime}, x_{s, 2}^{\prime}, \ldots, x_{s, n}^{\prime}\right\}, \quad s=1,2, \ldots, \sigma
$$

compare $(3 \cdot 0.4),(3 \cdot 0.5)$. Define the sets

$$
U^{\prime}=\left\{u_{1}^{\prime}, u_{2}^{\prime}, \ldots, u_{j}^{\prime}\right\}=U_{s=1}^{t} X_{s}^{\prime}
$$

and

$$
V^{\prime}=\left\{v_{1}^{\prime}, v_{2}^{\prime}, \ldots, v_{k}^{\prime}\right\}=\bigcup_{s=t+1}^{\sigma} X_{s}^{\prime}
$$

There exist scalars $a_{p, q} \in F$ such that

$$
u_{p}^{\prime}=\sum_{q=1}^{j} a_{p, q} u_{q}+\sum_{q=j+1}^{2} a_{p, q} q_{q-j}, \quad p=1,2, \ldots, j,
$$

and

$$
v_{p-j}^{\prime}=\sum_{q=1}^{j} a_{p, q} u_{q}+\sum_{q=j+1}^{l} a_{p, q} v v_{q-j}, \quad p=j+1, j+2, \ldots, ? .
$$

The matrix of coefficients $\left(\alpha_{p, q}\right)$ is nonsingular because both $X$ and $X^{\prime}$ are bases of the finite-dimensional linear space $K(D)$. Furthermore, from the definition of an optimally ranked basis it is easy to show that $\left(a_{p, q}\right)$ is block lower triangular with the s-th diagonal block having order $n_{s}$. Because of the block triangular structure, the determinant of $\left(a_{p, q}\right)$ is equal to the product of the determinants of the diagonal blocks. And because the determinant of $\left(\alpha_{p, q}\right)$ is nonzero, so is the determinant of every diagonal block. Therefore, the leading principal minor of order $j$ of the determinant of $\left(a_{p, q}\right)$ is nonzero. Let $\Delta_{j}$ denote this minor. 
Next, consider the Casorati matrix [X](i). It can be written in block form as

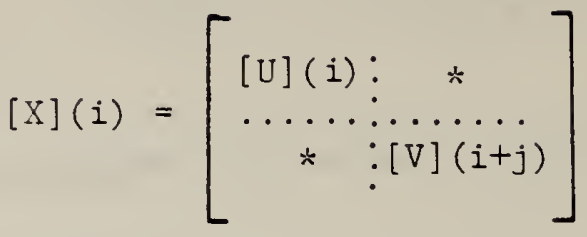

where the actual form of the blocks indicated by asterisks is unimportant. Similarly, we write

$$
\left[X^{\prime}\right](i)=\left[\begin{array}{cc}
{\left[U^{\prime}\right](i)} & * \\
\ldots \ldots \ldots \ldots \ldots & \ldots \ldots \ldots \\
* & \vdots
\end{array}\right] \text {. }
$$

If

$$
\left(\alpha_{p, q}\right)=\left[\begin{array}{ll}
A_{11} & 0 \\
\cdots \cdots & \ldots \\
A_{21} & A_{22}
\end{array}\right]
$$

is the corresponding block form of $\left(\alpha_{p, q}\right)$, we verify readily that

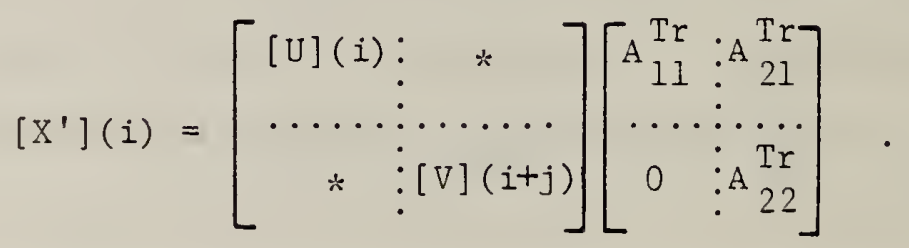

The leading principal minor of order $j$ of $\left|x^{\prime}\right|(i)$ is evidently given by

$$
\left|U^{\prime}\right|(i)=|U|(i) \cdot \operatorname{det}\left(A_{11}\right)
$$

Since $|U|(i) \neq 0$, by hypothesis, and $\operatorname{det}\left(A_{11}\right)=\Delta_{j} \neq 0$ we have $U^{\prime} \mid(i) \neq 0$. This completes the proof for $j<\ell$.

Einally, we note that the proof in the case $j=l$ follows by an argument analogous to that just used for $j<\ell$, except that there is no partitioning of matrices since $V$ and $V^{\prime}$ are empty. 


\subsection{Convergence}

Assume ${ }^{\dagger}$

$$
|u|(i) \neq 0
$$

and let $y$ be a solution of type $t$ of $(3.0 .1)$, where $0 \leq t \leq \sigma$. For each $n \geq m+1$ such that a solution $y_{n}$ of (3.0.1) exists satisfying both $(3.0 .2)$ and $(3.0 .3)$, there exist scalars $a_{1}(n), a_{2}(n), \ldots, a_{j}(n)$ and $\beta_{1}(n), \beta_{2}(n), \ldots, \beta_{k}(n)$ such that

$$
y_{n}=y+\sum_{s=1}^{j} \alpha_{s}(n) u_{s}+\sum_{s=1}^{k} \beta_{s}(n) v_{s} .
$$

In the case $t=\sigma$, we have

$$
y_{n}=y+\sum_{s=1}^{Z} a_{s}(n) u_{s}
$$

Then we prove readily, using $(3.0 .2)$ and $(3.2 .1)$, that $\alpha_{s}(n)=0$ for $s=1,2, \ldots, l$. Thus for each $n, y_{n}(r)=y(r)$ for $r=i, i+1, \ldots$. Ther efore we restrict the ensuing discussion to the cases in which $0 \leq t<c$.

Consider the set $C$ of all subsets of $k$ distinct sequences from $X$. We have $k>0$ by our assumption that $t<\sigma$. Obviousiy, $V=\left\{v_{1}, v_{2}, \ldots, v_{k}\right\} \in C$. We shall say that the optimally ranked basis $X$ is $\underline{j \text {-normal }}$ provided that $|V|(r) \neq 0$ for all sufficiently large $r$, and

$$
|\tilde{V}|(r)=o\{|V|(r)\} \text { as } r \rightarrow \infty
$$

for every $\tilde{V} \in C-\{V\}$. When $t=0, C=\{V\}$ and $X$ is automatically

The reader is referred to $\$ 3.0$ for notation and underlying assumptions. 
$j$-normal (0-normal). When $t>0$ the sets $\tilde{V}$ contain at least one sequence irom $U=\left\{u_{1}, u_{2}, \ldots, u_{j}\right\}$. In this case (3.2.3) expresses the quite reasonable condition that whenever one or more of the "dominant" solutions $v_{1}, v_{2}, \ldots, v_{k}$ is replaced in the Casoratian $|v|$ by a "subdominant" solution, then the resulting Casoratian is dominated by $|v|(r)$ as $r \rightarrow \infty$.

We now state and prove the convergence theorem. A similar result, but only for homogeneous linear difference equations, is given by Zahar $[27$, Th. 5.1]

Theorem 3.2.1 Assume $\mathrm{X}$ is a j-normal optimally ranked basis such that (3.2.1) is satisfied and $y(r)$ is a solution of (3.0.1) of type $t$. Also assume

$$
\lim _{r \rightarrow \infty} \frac{\left|V_{s}(y)\right|(r)}{|V|(r)}=0, \quad s=1,2, \ldots, k
$$

where $v_{s}(y)=\left\{v_{1}, \ldots, v_{s-1}, y, v_{s+1}, \ldots, v_{k}\right\}$. Then for sufficiently large $\mathrm{n}$ there exists a unique solution $\mathrm{y}_{\mathrm{n}}$ of $(3.0 .1)$ which satisfies $(3.0 .2)$ and $(3 \cdot 0.3)$. Fur thermore,

$$
\lim _{n \rightarrow \infty} y_{n}(r)=y(r)
$$

For each fixed value of $r \geq i+j$.

Proof: From $(3.2 .2),(3.0 .2)$ and $(3.0 .3)$ we derive the linear system

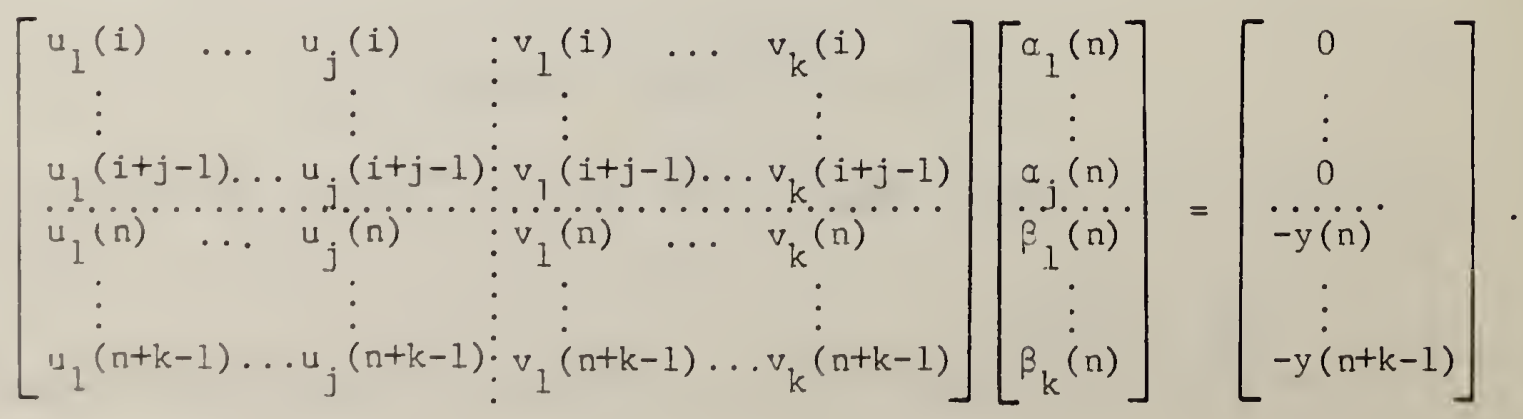


Although the block structure indicated in this system disappears when $t=0$, this modification ${ }^{\dagger}$ causes no difficulty in the following proof. Suppose $V$ were such that the upper right block of the matrix is zero (again, this is satisfied vacuously if $t=0$ ). Then the linear system becomes

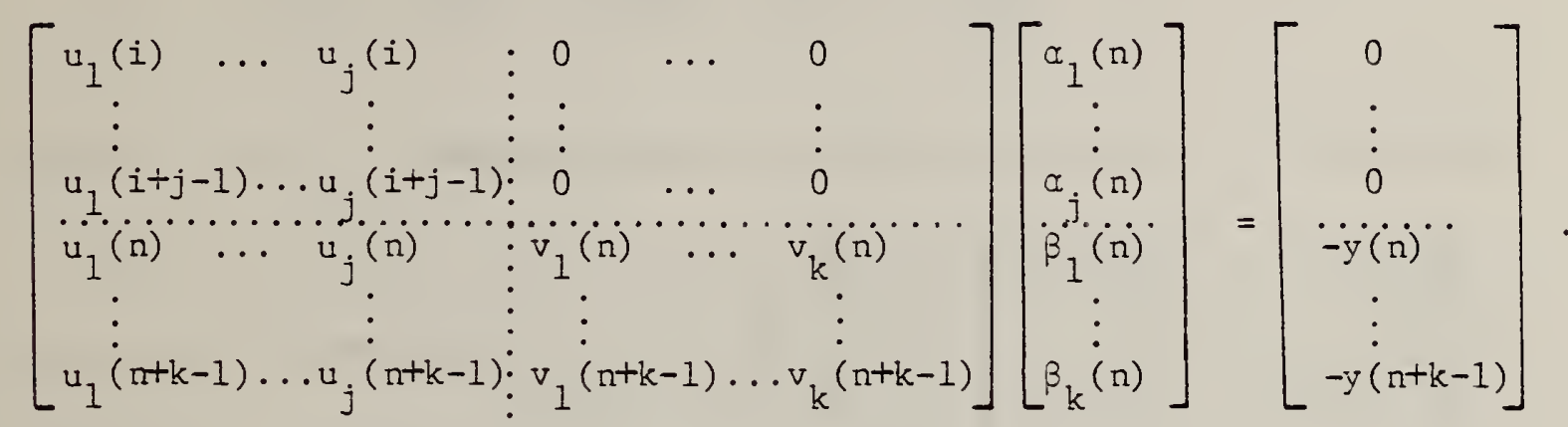

The determinant of this system is $|U|(i) \cdot|V|(n)$, and it is nonzero for all sufficiently large $\mathrm{n}$, in view of our assumptions. Using Cramer's rule and Laplace's general theorem on the expansion of determinants $[15, \S 93]$, we then find that

$$
\alpha_{1}(n)=\alpha_{2}(n)=\cdots=\alpha_{j}(n)=0
$$

and

$$
\beta_{s}(n)=-\frac{\left|V_{s}(y)\right|(n)}{|V|(n)}, \quad s=1 ; 2, \ldots, k .
$$

Therefore, for all $\mathrm{r}$ and for all sufficiently large $\mathrm{n}$, we have

$$
y_{n}(r)=y(r)-\sum_{s=1}^{k} \frac{\left|v_{s}(y)\right|(n)}{|v|(n)} v_{s}(r)
$$

The other case in which the block structure disappears, which is $t=c$, was disposed of earlier. 
Then (3.2.5) follows from (3.2.4).

In order to complete the proof, let us introduce the sequences (3.2.7) $\quad v_{p}^{\prime}=v_{p}+\gamma_{p, I} u_{1}+\cdots+\gamma_{p, j} u_{j}, p=1,2, \ldots, k$,

where the $\gamma_{p, q}$ are to be chosen in such a way that

$$
v_{p}^{\prime}(i+r)=0, \quad r=0,1, \ldots, j-1 .
$$

Thus the $r_{p, q}$ have to satisfy the linear systems

$$
\left[\begin{array}{ccc}
u_{1}(i) & \ldots & u_{j}(i) \\
\vdots & & \vdots \\
u_{I}(i+j-1) & \ldots & u_{j}(i+j-1)
\end{array}\right]\left[\begin{array}{c}
r_{p, I} \\
\vdots \\
\gamma_{p, j}
\end{array}\right]=\left[\begin{array}{c}
-v_{p}(i) \\
\vdots \\
-v_{p}(i+j-1)
\end{array}\right], p=1,2, \ldots, k
$$

each of which is nonsingular because $|U|(i) \neq 0$, by hypothesis. Let $V^{\prime}=\left\{v_{1}^{\prime}, v_{2}^{\prime}, \ldots, v_{k}^{\prime}\right\}$. Clearly it suffices to show that $U U V^{\prime}$ is a $j$-normal optimally ranked basis for $D$ such that

$$
\lim _{r \rightarrow \infty} \frac{\left|V_{s}^{\prime}(y)\right|(r)}{\left|V^{\prime}\right|(r)}=0, \quad s=1,2, \ldots, k
$$

Since $v_{p}$ dominates every sequence in $U$, we have from $(3.2 .7)$

$$
\frac{v_{p}^{\prime}(r)}{v_{p}(r)} \rightarrow 1 \text { as } r \rightarrow \infty
$$

for each $p, l \leq p \leq k$. Therefore $v_{p}^{\prime}$ has the same type as $v_{p}$. Furthermore, we may verify the three conditions of Definition 2.2.1, as follows:

$$
\text { 1) } \quad \frac{u(r)}{v_{p}^{\prime}(r)}=\frac{u(r)}{v_{p}(r)} \cdot \frac{v_{p}(r)}{v_{p}^{\prime}(r)} \rightarrow 0 \text { as } r \rightarrow \infty
$$

for every $u \in U$, and 


$$
\frac{v_{p}^{\prime}(r)}{v_{q}^{\prime}(r)}=\frac{v_{p}^{\prime}(r)}{v_{p}(r)} \cdot \frac{v_{p}(r)}{v_{q}(r)} \cdot \frac{v_{q}(r)}{v_{q}^{\prime}(r)} \rightarrow 0 \text { as } r \rightarrow \infty
$$

whenever type $\left(v_{p}^{\prime}\right)<$ type $\left(v_{q}^{\prime}\right)$;

ii) $\frac{v_{p}^{\prime}(r)}{v_{q}^{\prime}(r)}=\frac{v_{p}^{\prime}(r)}{v_{p}(r)} \cdot \frac{v_{p}(r)}{v_{q}(r)} \cdot \frac{v_{q}(r)}{v_{q}^{\prime}} \frac{v_{p}(r)}{v_{q}}-\frac{v_{q}(r)}{v_{q}}$ as $r \rightarrow \infty$

whenever type $\left(v_{p}^{\prime}\right)=$ type $\left(v_{q}^{\prime}\right)$;

(iii) Let $v_{p}^{\prime}, v_{p+1}^{\prime}, \ldots, v_{q}^{\prime} \in v^{\prime}$ be all the sequences of one type, and let $v^{\prime}$ be any fixed one of these. Let the corresponding unprimed sequences be $v, v_{p}, v_{p+1}, \ldots, v_{q} \in V$. Take $\delta_{p}, \delta_{p+1}, \ldots, \delta_{q} \in F$, not all zero. For each $s=p, p+1, \ldots, q$ we have

$$
\frac{\delta_{s} v_{s}^{\prime}}{v^{\prime}}=\frac{\delta_{s}\left(v_{s}+\gamma_{s, 1} u_{1}+\cdots+r_{s, j_{j}} u^{\prime}\right)}{v^{\prime}}=\frac{\delta_{s} v_{s}}{v^{\prime}}+o(1)
$$

(since $u_{1}, u_{2}, \ldots, u_{j}$ are of lower type than $v^{*}=v\{1+o(1)\}$ ). Hence

$$
\frac{\delta_{p} v_{p}^{\prime}+\cdots+\delta q^{\prime} q}{v^{\prime}}=\frac{\delta_{p} v_{p}+\cdots+\delta q^{\prime} q}{v^{\prime}}+o(1)
$$

รurthermore

$$
\frac{\delta v_{p}^{\prime}+\cdots+\delta q^{\prime} v^{\prime}}{v^{\prime}}=\frac{\delta p p+\cdots+\delta q v}{v}\{1+o(1)\}+o(1)
$$

from which it is clear that

$$
\lim _{r \rightarrow \infty}\left|\sum_{s=p}^{q} \delta v_{s}^{\prime}(r) / v^{\prime}(r)\right|>0
$$

Thus, $U \cup V^{\prime}$ is an optimally ranked basis. 
Next we prove that $\left|V^{\prime}\right|(r) \neq 0$ for all sufficiently large $r$. We recall that a determinant is a multilinear function of its columns. If we express

$$
v^{\prime}(r)=\left|v_{1}^{\prime}, v_{2}^{\prime}, \ldots, v_{k}^{\prime}\right|(r)
$$

where $v_{1}^{\prime}, v_{2}^{\prime}, \ldots, v_{k}^{\prime}$ temporarily denote the successive columns of $\left|V^{\prime}\right|(r)$, then we derive

$$
\begin{aligned}
& \left|v^{\prime}\right|(r)=\left|v_{1}, v_{2}^{\prime}, \ldots, v_{k}^{\prime}\right|(r)+\sum_{q=1}^{j} r_{1, q}\left|u_{q}, v_{2}^{\prime}, \ldots, v_{k}^{\prime}\right|(r) \\
& =\left|v_{1}, v_{2}, v_{3}^{\prime}, \ldots, v_{k}^{\prime}\right|(r)+\sum_{q=1}^{j} r_{1, q}\left|u_{q}, v_{2}^{\prime}, \ldots, v_{k}^{\prime}\right|(r) \\
& +\sum_{q=1}^{j} r_{2, q}\left|v_{1}, u_{q}, v_{3}^{\prime}, \ldots, v_{k}^{\prime}\right|(r) \\
& =\cdots \text {, }
\end{aligned}
$$

and hence

$$
\begin{aligned}
\left|v^{\prime}\right|(r)=|v|(r) & +\sum_{q=1}^{j} r_{1, q}\left|u_{q}, v_{2}^{\prime}, \ldots, v_{k}^{\prime}\right|(r) \\
& +\sum_{q=1}^{j} r_{2, q}\left|v_{1}, u_{q}, v_{3}^{\prime}, \ldots, v_{k}^{\prime}\right|(r) \\
& +\ldots \\
& +\sum_{q=1}^{j} r_{j, q}\left|v_{1}, v_{2}, \ldots, v_{k-1}, u_{q}\right|(r) .
\end{aligned}
$$

If we continue this construction until every reference to a primed $v$ is removed, then it is clear that $\left|V^{\prime}\right|(r)$ is equal to the sum of $|V|(r)$ plus a linear combination of determinants formed Erom $|V|(r)$ by replace- 
ment of at least one $v$ by $a$. By our assumption that $X$ is $j$-normal, we conclude

$$
\left|V^{\prime}\right|(r)=|V|(r)\{1+o(1)\} \text { as } r \rightarrow \infty .
$$

Since $|V|(r) \neq 0$ for sufficiently large $r$, we also have $\left|V^{\prime}\right|(r) \neq 0$ for sufficiently large $r$.

If one or more of the solutions $v_{1}^{\prime}, v_{2}^{\prime}, \ldots, v_{k}^{\prime}$ are replaced in $\left|V^{\prime}\right|(r)$ by subdominant solutions from $U$, then a construction like the one preceding shows that the modified Casoratian $\left|\tilde{V}^{\prime}\right|(r)$ is dominated by $|V|(r)$ as $r \rightarrow \infty$, since the original optimally ranked basis is $j$-normal. Consequently, using (3.2.9), we conclude $U \in V^{\prime}$ is $j$-normal. A similar argument shows that (3.2.8) is satisfied, and the theorem is proved. 


\subsection{Stability}

Let us consider solutions of $(3.0 .1)$ of type $t$, where $0 \leq t<\sigma$.

Let $j$ be the dimension of the subdominant subspace of type $t$ for $D$. Let $i$ be a point at which $|U|(i) \neq 0$; see $(3 \cdot 0.7)$. Suppose $D$ is $(i, j)$-factorizable; see Definition 1.2.1. Let $A^{i}=\left[a_{0}^{i}, a_{1}^{i}, \ldots a_{j}^{i}\right]$ be a leit factor and $B^{i+j}=\left[b_{0}^{i+j}, b_{1}^{i+j}, \ldots, b_{k}^{i+j}\right]$ the corresponding right Factor of $D_{j}^{i}$, so that $D_{j}^{i}=A_{j}^{i} B^{i+j}$ is an (i,j)-factorization of $D$.

The next theorem provides a generalization of Theorem 1.3.2, from totaliy separable linear difference equations to equations that are merely separable. The renark following Theorem 1.3 .2 applies here as well. That is, the back substitution stage of the algorithm is stable, at least for sufficiently large $r$.

Theorem 3.3.I If $|U|(i) \neq 0$ and $D_{j}^{i}=A_{j}^{i} B^{i+j}$, then the difference operator $B^{i+j}$ is separable and any solution $y$ of (3.0.1) of type $t$ or less is a sequence of type zero with respect to $B^{i+j}$.

Proof: Suppose $j=0$, i.e., type $(y)=0$ with respect to $D$. Then $A^{i}=\left[a_{0}^{i}\right]$ and $B^{i}=\left[b_{0}^{i}, b_{1}^{i}, \ldots, b_{2}^{i}\right]$ and we have $D^{i}=A^{i} B^{i}$.

Now let $x \leq x$, where $x$ is the optimally ranked basis (3.0.4). Then $D x=0$; hence $D^{i} x^{i}=A^{i} B^{i} x^{i}=0$. This last equation is equivalent to

$$
a_{0}(r) \sum_{s=0}^{\ell} b_{s}(r) x(r+s)=0, r=i, i+1, \ldots .
$$

Since $a_{0}^{i}$ is free of zeros by the definition of $(i, j)$ - factorizability, we have $B^{i} x^{i}=0$. Thus $\left\{x^{i} \mid x \in X\right\}$ is an optimally ranked basis for $B^{i}$ and it follows immediately that type $(y)=0$ with respect to $B^{i}$.

\footnotetext{
The case $t=\sigma$ corresponds to a maximal solution of ( 3.0 .1$)$. Since the general algorithm reduces to pure forward recurrence in this case, it is stable; see jैl.l.
} 
Next suppose $j$ satisfies $0<j<\ell$. Let $v \in V$, where $V$ is given by $(3.0 .8)$. In view of our assumption that $|U|(i) \neq 0$, we may assume that

$$
v(i+r)=0, \quad r=0,1, \ldots, j-1 ;
$$

compare the proof of Theorem 3.2.1. Since $v \in K(D)$ we have $D v=0$. It follows that for each $n \geq i+j+\max (j, k)$ the subsequence $v^{i, n+k-1}$ satisfies the finite boundary value problem

$$
D_{j}^{i, n-j-1} v_{v}^{i+j, n-1}=\left[\begin{array}{c}
\ldots \ldots \ldots \ldots \ldots \ldots \ldots \\
-D \\
-n-\ell, n-j-1 \\
v, n+k-1
\end{array}\right]
$$

compare (1.2.4). For each $\mathrm{n}$, these boundary value problems are nonsingular and factorizable as shown by Theorem 1.2.1.

Therefore, Theorem 1.2 .2 is applicable and we see that $v^{i+j \cdot n-1}$ satisfies

$$
B_{0}^{i+j, n-1} v^{i+j, n-1}=-\left[\begin{array}{c}
\ldots \\
\ldots \ldots \ldots \ldots \\
B_{k}^{n-k, n-1}{ }_{v}^{n, n+k-i}
\end{array}\right]
$$

for each $n \geq i+j+\max (j, k) ; \operatorname{compare}(1.2 .7)$ and the remark following the proof of Theorem 1.2.2. The last equation is equivalent to

$$
\sum_{s=0}^{k} b_{s}(r) v(r+s)=0, \quad r=i+j \cdot i+j+1, \ldots n-1 .
$$

Since $\mathrm{n}$ may be arbitarily large, we have

$$
\sum_{s=0}^{k} b_{s}(r) v(r+s)=0, \quad r=i+j, i+j+1, \ldots
$$

or, equivalently, $B^{i+j}{ }_{v}^{i+j}=0$. Therefore, $\left\{v^{i+j} \mid v \in v\right\}$ is an optimally ranked basis for $B^{i+j}$. Since $y$ is dominated by every sequence in $V$, 
it follows that type $(y)=0$ with respect to $B^{i+j} \cdot \mid$

Now let us turn to the forward elimination stage of the algorithm. This consists of applying a finite number of steps of Gaussian elimination, without pivoting, to the infinite linear system

$$
D_{j}^{i} y^{i+j}=g^{i}-\left[\begin{array}{r}
D_{0}^{i, i+j-1} y^{i, i+j-1} \\
0
\end{array}\right]
$$

compare (1.3.6). Rounding errors introduced in this process are equivalent to perturbations in the original problem. Thus, instead of satisfying (3.3.1) the computed solution $\tilde{y}^{i+j}$, say, is an exact solution of a system of the form

$$
\left(D_{j}^{i}+E_{j}^{i}\right) \tilde{y}^{i+j}=g^{i}-\left[\begin{array}{r}
D_{0}^{i, i+j-1} 1_{y}^{i}, i+j-1 \\
0 \ldots \ldots \ldots \ldots
\end{array}\right]+e^{i}
$$

where the terms $E_{j}^{i}$ and $e^{i}$ represent the perturbation.

The effect of introducing a single element of $e(r)$, say at $r=s$, is to perturb the true solution $y^{i+j}$ by a linear combination of the solutions of the corresponding homogeneous equation. Because of the boundary conditions we have imposed "at infinity", only the solutions $u_{1}, u_{2}, \ldots u_{j}$ enter from the optimally ranked basis $\left\{u_{1}, u_{2}, \ldots, u_{j}, v_{1}, v_{2}\right.$, $\left.\ldots, v_{k}\right\}$; compare equation (3.0.3) and Theorem 3.2.1. Subsequently, we have only to consider what happens when $r>s$, and here it will be the multiple of $u_{j}$ that will be the fastest growing.

The effect of $E_{j}^{i}$, also, can be allowed for by making a perturbation of the right side. To terms of the first order, we have

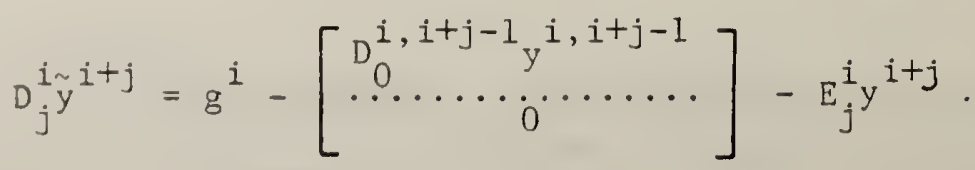


Then the arguments of the preceding paragraph again apply.

Since the wanted solution $y$ is of type $t$, we conclude that the process of forward elimination is stable in the sense that each perturbation subsequently grows at a rate that does not exceed that of the wanted solution. Of course, there will be a loss of accuracy when an element of $E_{j}^{i}$ (or $e^{i}$ ) is large compared with the rounding error in the stored value of the corresponding element of $D_{j}^{i}$ (or of the right side of (3.3.1)). This may occur, for example, when there is heavy cancellation in the formation of an element that is subsequently used as a pivot. However, the important point is that the effect of each such loss is not magnified in subsequent steps of the algorithm.

An extension of the foregoing discussion shows that if we select a value of $j$ (that is, the number of prescribed initial conditions) that is less than the dimension of the subdominant subspace of the wanted solution, then the forward elimination remains stable. However, the back-substitution is now unstable; compare the proof of Theorem 3.3.1. Similarly if $j$ is too large then the forward elimination is unstable, and the back-substitution is stable. Nevertheless if, in practice, the actual instabilicies are weak owing to weak separation of solutions of consecutive types as $r \rightarrow \infty$, then it may be advantageous to select a value of $j$ that differs from the dimension of the subdominant subspace of the vanted solution. This is because the loss of accuracy caused by the instability is offset by a substantial reduction in the value of the terminal point $n$ owing to increased convergence. This modification is illustrated by numerical examples in Chapter 4 . 


\subsection{Introduction}

Several numerical examples will be given to illustrate the general algorithm. The examples all involve fourth-order linear difference operators with nonconstant coefficients. Both homogeneous and inhomogeneous difference equations are treated.

The fourth-order operators were produced by using the method described in $[14, \$ 12.22]$, starting with two second-order recurrence relations which have as solutions Bessel functions, modified Bessel functions or associated Legendre functions. The inhomogeneous equations have as particular solutions Anger-Weber functions or Struve functions. The right sides of these equations were produced as a by-product of the method used to produce the fourth-order operators.

The production of the fourth-order difference operators from the corresponding pairs of second-order operators requires only elementary algebraic manipulations. These calculations are rather lengthy, however, and would be extremely difficult (as well as tedious) to complete accurately by hand. Instead, the MACSYMA symbol manipulation system, described in [12], was employed ${ }^{\dagger}$. This procedure had the added advantage of storing the required formulas for the coefficients directly in the computer, ready For numerical evaluation, thereby further reducing the possibility of human error. Similarly, MACSYMA was used to advantage in the production of the right sides for the inhomogeneous examples.

Development of which is currently supported, in part. by the United States Department of Energy under Contract Number DE-AC02-79ER10357 and by the Viational Aeronautics and Space Administration under Contract Number NSG1321. 
In the case of the Bessel functions $\mathrm{J}_{\mathrm{r}}(\mathrm{x}), \mathrm{Y}_{\mathrm{r}}(\mathrm{x})$ and modified Bessel functions $I_{r}(x), K_{r}(x)$ we restrict ourselves to integer order $r \geq 0$ and real argument $x>0$. Relevant properties of these functions are given in [16, Chapter 9]. For example, for fixed $x$ the functions $\mathrm{J}_{\mathrm{r}}(\mathrm{x})$ and $\mathrm{Y}_{\mathrm{r}}(\mathrm{x})$ satisfy the linear recurrence relation

$$
y(r-1)-\frac{2 r}{x} y(r)+y(r+1)=0
$$

Similarly, $I_{r}(x)$ and $(-)^{r} K_{r}(x)$ satisfy

$$
y(r-1)-\frac{2 r}{x} y(r)-y(r+1)=0
$$

The Anger-Weber functions $E_{r}(x)$ satisfy

$$
y(r-1)-\frac{2 r}{x} y(r)+y(r+1)=-\frac{2\left\{1-(-1)^{r}\right\}}{\pi x} ;
$$

and the Struve functions $\mathrm{H}_{\mathrm{r}}(\mathrm{x})$ satisfy

(4.0.4) $y(r-1)-\frac{2 r}{x} y(r)+y(r+1)=\frac{\left(\frac{1}{2} x\right)^{r}}{\sqrt{\pi} \Gamma\left(r+\frac{3}{2}\right)}$

compare (4.0.1) in both cases. Relevant properties of the $E_{r}(x)$ and $H_{r}(x)$ appear in Olver's paper [21] where they were used as examples for second-order inhomogeneous equations.

The coefficients of the fourth-order homogeneous recurrence relation obtained from (4.0.1) with $x_{1}=x_{1}$ and $(4.0 .2)$ with $x=x_{2}$ are shown in Figure 6 in the form of FORTRAN statements produced by MACSYMA. In addition, Figure 6 shows the right-hand sides corresponding to $E_{r}\left(x_{1}\right)$ and $\mathrm{H}_{\mathrm{r}}\left(\mathrm{x}_{1}\right)$. We designate the difference operator $\mathrm{D}$ defined by these 


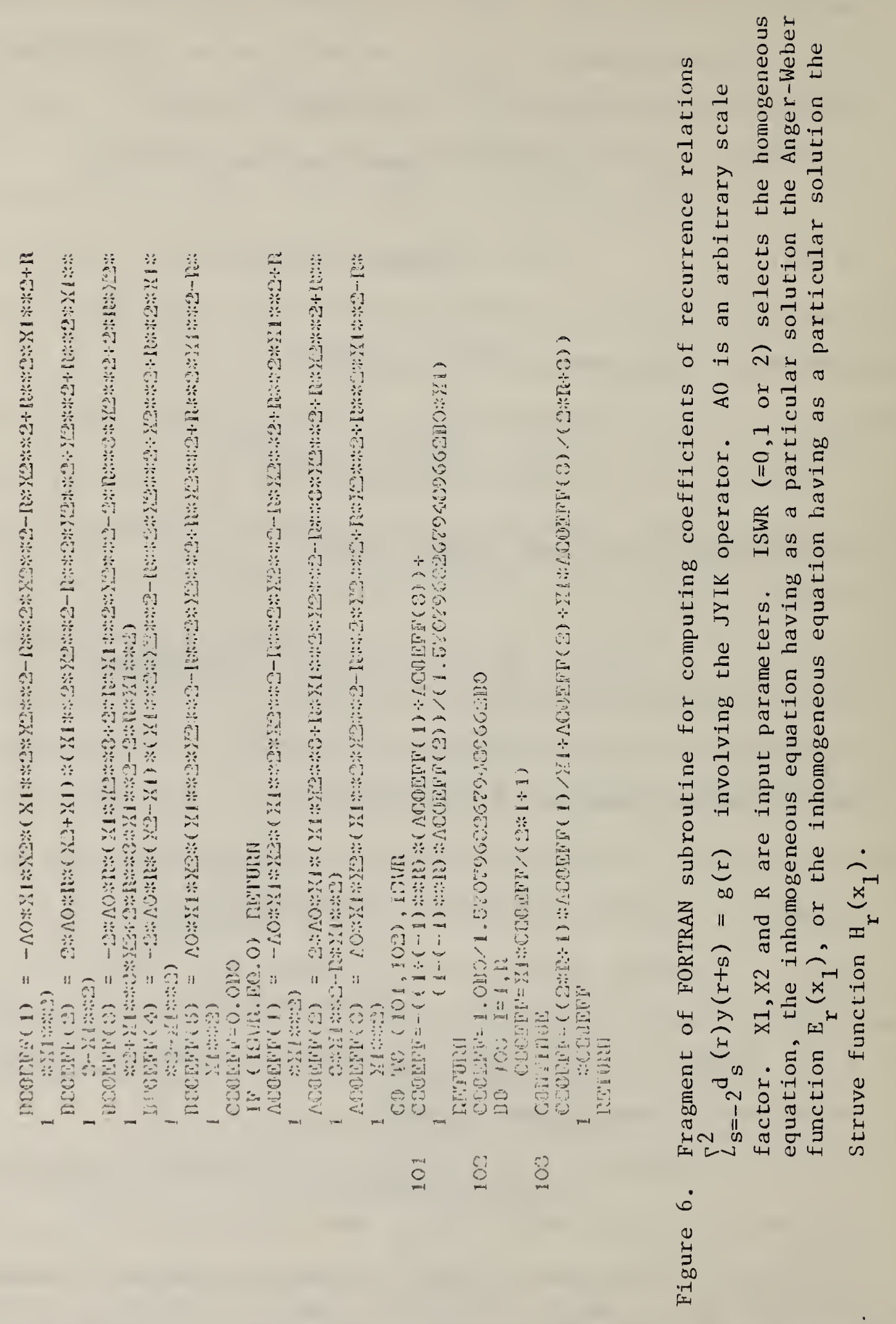


coefficients the JYIK operator. Since the functions $\mathrm{J}_{\mathrm{r}}\left(\mathrm{x}_{1}\right), \mathrm{Y}_{\mathrm{r}}\left(\mathrm{x}_{1}\right)$, $I_{r}\left(x_{2}\right),(-){ }_{K}{ }_{r}\left(x_{2}\right)$ are linearly independent, they form a fundamental set of solutions of $\mathrm{Dy}=0$.

Similarly in the case of the associated Legendre functions $\mathrm{P}_{\mathrm{r}}^{\mathrm{H}}(\mathrm{x})$, $Q_{r}^{\mu}(x)$ we restrict consideration to integer degree $r \geq 0$, integer order $\mu \geq 0$, and real argument $x>0$. Relevant properties of these functions may be found in [16, Chapter 8]. We use the r-wise linear recurrence relation

$$
(r+\mu) y(r-1)-(2 r+1) x y(r)+(r-\mu+1) y(r+1)=0
$$

valid for fixed $x$ and $\mu$. The coefficients of the fourth-order difference operator D, shown in Figure 7, were produced (using MACSYMA) from (4.0.1) with $x=x_{1}$ and $(4.0 .5)$ with $x=x_{2}$. Figure 7 also shows the right sides corresponding to $\mathrm{E}_{\mathrm{r}}\left(\mathrm{x}_{1}\right)$ and $\mathrm{H}_{\mathrm{r}}\left(\mathrm{x}_{1}\right)$. In this case the functions $J_{r}\left(x_{1}\right), Y_{r}\left(x_{1}\right), P_{r}^{\mu}\left(x_{2}\right), Q_{r}^{\mu}\left(x_{2}\right)$ form a fundamental set of solutions of $D y=0$. Accordingly, we designate $D$ the JYPQ operator.

A FORTRAN program has been written to implement the general algorithm. The user may select either the JYIK operator or the JYPQ operator. He may also elect to solve the homogeneous equation, the inhomogeneous equation having the Anger-Weber function as a solution, or the inhomogeneous equation having the Struve function as a solution. Alternatively, he may provide a subroutine which generates the coefficients of an arbitrary linear difference equation of any order.

The user supplies the program with the starting point $i$ and the terminal point $m$ of the desired subsequence of the solution to be computed, the number $j$ of initial values, the subsequence $y^{i, i+j-1}$ of initial values, and the parameters $\nu$ and $\varepsilon$ for use in the convergence test (to be described 

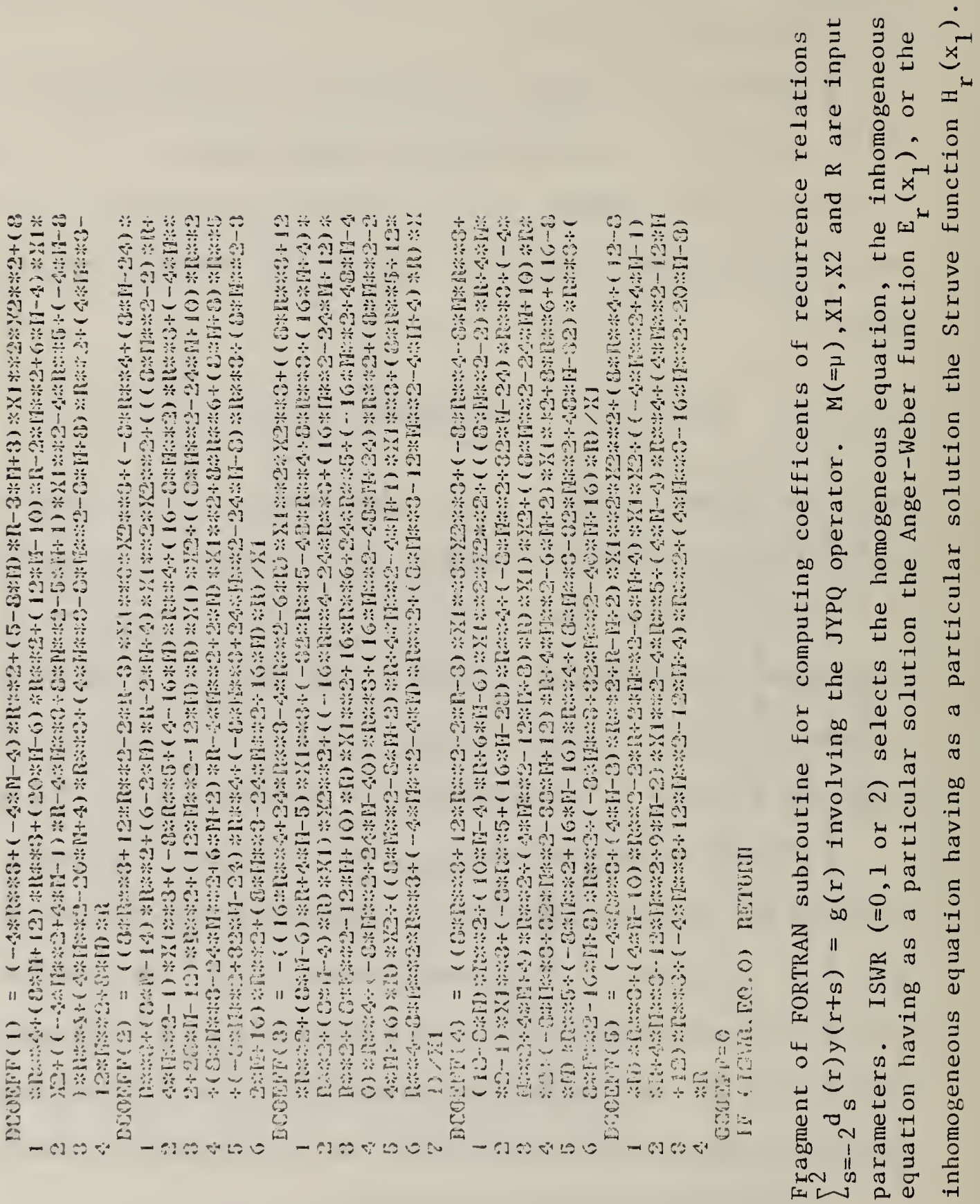

$\underset{\sim}{0}$ 


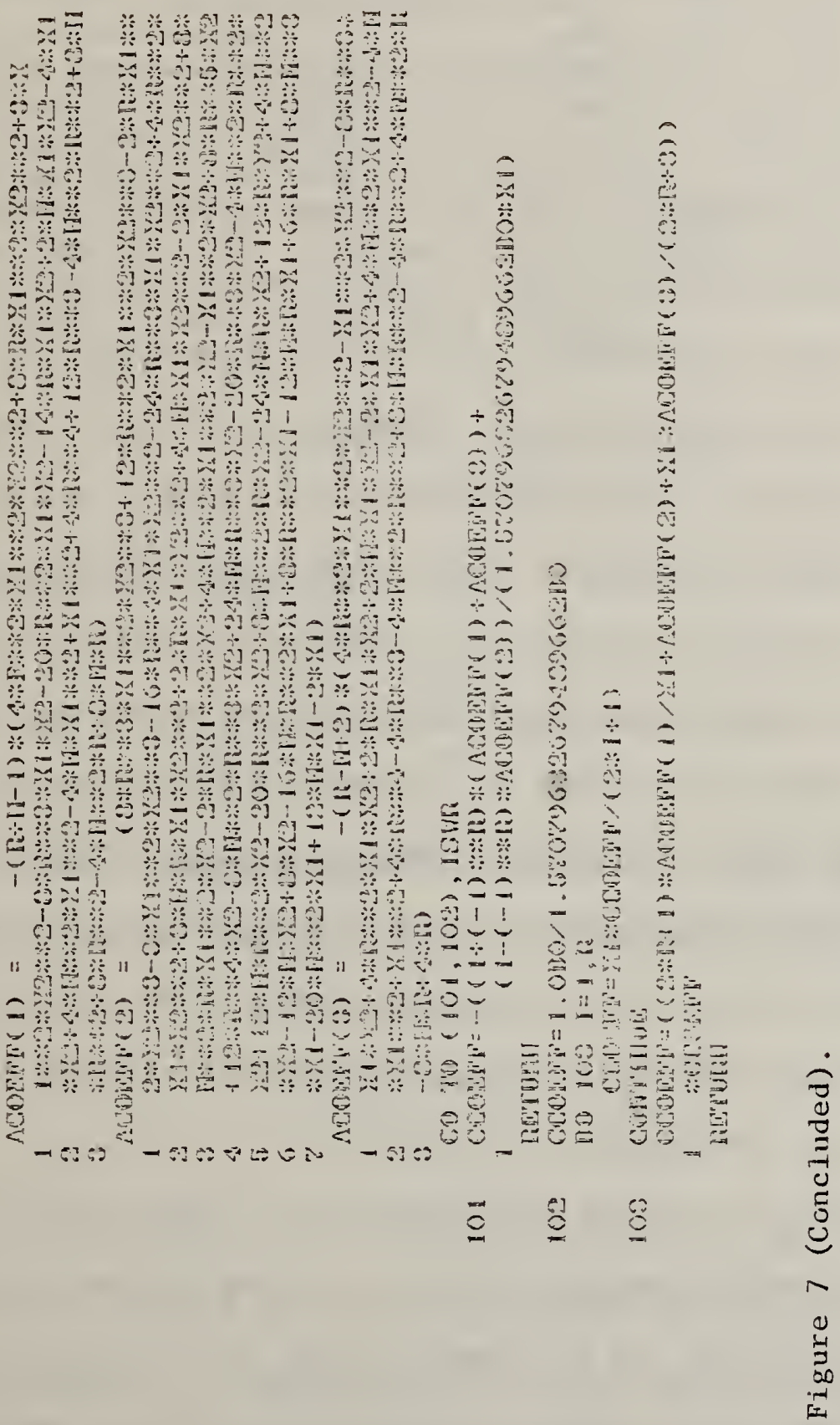


below). The program solves the proposed problem by executing three phases. In the initial phase the first $m+1+v-i-j$ rows of the infinite linear system (1.3.6) are stored. Forward elimination without pivoting is performed on these rows. This results in the storing of the first $m+1+v-i-j$ rows of the left and right factors of the $(i, j)-$ factorization $D_{j}^{i}=A_{j}^{i} B^{i+j}$, and also the subsequence $z^{i+j, m+v}$ of the solution of (1.3.8). The elimination procedure is carried out by modifications, described below, of the subroutines DGBFA and DGBSL which are available in the mathematical software package known as LINPACK; see [ 8 ].

The unmodified DGBFA performs forward elimination with partial pivoting on a finite band tnatrix, ignoring the right side. It produces an upper triangular matrix, a lower triangular matrix and a vector of pivoting information. These results may then be used by the unmodified DGBSL to solve the corresponding Iinear system with specified right side. Aternatively, the user can direct that DGBSL solve a linear system having as its matrix of coefficients the transpose of the matrix input to DGBFA. Provision has been made in DGBFA to suppress the partial pivoting. When this is done the vector of pivoting information is superfluous and (except For rounding errors) the matrix obtained by premultiplying the upper triangular matrix by the lower triangular matrix is the original band matrix. This factorization is therefore of exactly the kind required by Definition 1.2.1; see equation (1.2.5).

When partial pivoting has been suppressed in DGBFA, DGBSL proceeds, in effect, by forward elimination without pivoting followed by back substitution. Provision has been made in DGBSL to exit after the forward elimination stage and to re-enter at the start of the back substitution stage. Thus the user has the option of examining the result of the 
forward elimination stage and perhaps electing to continue it without having to do any back substitution. All of the above modifications of DGBFA and DGBSL, to provide just the tools that are needed in our algorithm, were easily achieved because of the clarity and flexibility of the coding and documentation of LINPACK.

We return to the description of the initial phase. The next computation is the subsequence $\mathrm{w}_{\mathrm{m}}^{\mathrm{m}+1, \mathrm{~m}+v}$; compare equations $(1.3 .16)$ and (1.3.17) with $s=m$. Since $w_{m}^{m-k+1}$ satisfies the adjoint equation, it is produced using the forward elimination stage of the (modified) subroutine DGBSL in which the input matrix to DGBFA is automatically transposed. Finally, the initial phase computes

$(4.0 .5) \quad y_{r}(m)=\sum_{s=m}^{r-1} w_{m}(s) z(s) / b_{0}(m)$

for $r=m+1, m+2, \ldots, m+v, m+v+1$ and

$$
\eta_{r, v}(m)=\sum_{s=r}^{r+v-1} w_{m}(s) z(s) / b_{0}(m)
$$

for $r=m+1$. Equation $(4.0 .6)$ is the same as (1.3.18) apart from a change of symbols. Equation $(4.0 .7)$ is the approximation to the truncation error

$$
\eta_{r}(m)=y(m)-y_{r}(m)
$$

which is obtained when $y(m)$ is replaced by $y_{r+v}(m)$ : compare $(1.3 .20)$. After completion of the initial phase, the program proceeds to the iteration phase. One additional row of the infinite linear system (1.3.6) is stored. Since the previously determined part of the $(i, j)$-factorization has replaced the corresponding rows of the original matrix in the computer 
storage, these rows are no longer available to extend the forward elimination stage. Therefore, extension of the forward elimination is accomplished by an alternative procedure that requires the solutions of two triangular linear systems of orders $j$ and $k$. This is achieved using DTRSL, a LINPACK subroutine that solves triangular systems. Next, the formerly obtained subsequences of the solutions $z^{i+j}$ and $w_{m}^{m+1}$ are extended to include the next higher term. Then the series $(4.0 .6)$ and $(4.0 .7)$ are summed with the new value of $r$, and the following convergence test is applied: If

$$
\left|\eta_{r, v}(m)\right| \leq \varepsilon\left|y_{r+v}(m)\right|
$$

then the program proceeds to the third and final phase; otherwise, the iteration phase is repeated and the convergence test re-applied.

The final phase of the program is the back substitution stage of the algorithm. Let $n$ denote the value of $r$ which satisfies $(4.0 .8)$. The back substitution is begun at this point, using the (modified) subroutine DGBSI. This yields the computed solution $y_{n}^{i+j, n-1}$ of $(1.3 .7)$ and also the desired approximation of $y(r)$ over the range $r=i+j, i+j+1, \ldots, m$.

In all of the examples presented in this thesis the chosen termination criterion corresponds to an estimated relative error of $10^{-10}$ in the final term $y_{n}(m)$ of the requested sequence of values of the function. Thus $\equiv=10^{-10}$ in equation $(4 \cdot 0.8)$. For the values of $x_{1}$ and $x_{2}$ selected in the examples the solutions are reasonably well-separated as $r \rightarrow \infty$, hence we take the "testing parameter" $v$ in equation $(4.0 .7)$ to be 1 throughout. Initial values were entered to 10 significant figures in every case. All computations were performed internally to 18 figures 
and the output printed to 10 figures. For each example we present (i) the solution $z(i+j), z(i+j+1), \ldots, z(n)$ of equation (1.3.8) (the forward elimination stage); (ii) the solution $w_{m}(m+1), w_{m}(m+2), \ldots, w_{m}(n)$ of $(1.3 .16),(1.3 .17)$ (the forward recurrence of the adjoint equation for use in the termination criterion); (iii) the sequence $y_{m+1}(m), y_{m+2}(m), \ldots$, $\mathrm{y}_{\mathrm{n}+\mathrm{v}}(\mathrm{m})$ of approximants to the value $\mathrm{y}(\mathrm{m})$ (see equation $(4.0 .6)$ ); (iv) the sequence $\eta_{m+1,1}(m), \eta_{m+2,1}(m), \ldots, \eta_{n, 1}(m)$ of estimates of the truncation error incurred in accepting the terminal points of $m+1, m+2, \ldots, n$ respectively (see equation $(4.0 .7)) ;(v)$ the sequence $y_{n}(n-1), y_{n}(n-2), \ldots$, $y_{n}(i+j)$ derived from equation (1.3.7) (the back substitution stage).

The initial values that were used in the boundary value problem actually solved in each example are shown in parentheses in the tables; see, for example, Table 5. The terminal values (which are all zero) are also shown. Forward recurrence of the $j$-th order difference equation $A^{i} z^{i}=g^{i}$ starting from the given initial values produces a solution of (1.3.8) (the algorithm produces this solution by forward elimination). Similarly, backward recurrence of the $k-t h$ order difference equation $B^{i+j} y_{n}^{i+j}=z^{i+j}$ starting from the cerminal values (which are ail zero) produces the corresponding solution of the boundary value problem; this is the back substitution stage. Data is given in the tables only for selected values of $r$. All of the values of $r$ that were used beyond $r=m$ are included, however. These values illustrate the termination procedure.

It should be noted that when $j=4$ the method as implemented by the FORTRAN program is equivalent to, but not identical with, forward recurrence of the given difference equation. See, for example, Table 8 . This is because DGBFA, the LINPACK matrix factorization subroutine, scales the linear system in such a way 
that the principal diagonal of the lower triangular factor is a sequence of ones. Therefore, the result of the forward elimination stage differs from the result of the back substitution stage (unless the trailing coefficients of the original linear difference operator are all equal to one). Since there is no question of truncation error when $j=4$, the sequences $w_{m}(r), y_{r}(m)$ and $\eta_{r, l}(m), r=m+1, m+2, \ldots$, are not defined.

It has been observed that Olver's original second-order method is likely to cause overflow in most computers. This same likelihood of overElow exists for the extended algorithm. The examples to be presented in this chapter were computed using double precision on a Univac 1108 . Double precision was used not for higher precision but for wider exponent range. The double precision range extends to $10^{308}$ whereas the largest number generated in the examples was somewhat greater than $10^{200}$. Van der Cruyssen [25] gives a rescaled version of the second-order algorithm, based on LU decomposition, that greatly reduces the incidence of overflow or underflow. An equivalent procedure for overcoming this difficulty, based directly on the use of ratios of consecutive terms in each solution, is described by Aggarwal and Burgmeier [1]. In the present writer's view, however, it is preferable (if possible) to avoid mathematical modifications of algorithms simply to suit arbitrary limitations of existing computers. A software alcernative to rescaling is proposed in [24], in whicn a full integer storage location is allocated to the exponent of each floating-point number. A FORTRAN implementation of this proposal is available; see [1.1]. 


\subsection{Examples Involving the Homogeneous JYIK Equation}

From the asymptotic forms

(4.1.1) $\quad J_{r}(x) \sim \frac{1}{(2 \pi r)^{1 / 2}}\left(\frac{e x}{2 r}\right)^{r}, \quad Y_{r}(x) \sim-\left(\frac{2}{\pi r}\right)^{1 / 2}\left(\frac{e x}{2 r}\right)^{-r}$

and

$(4.1 .2) I_{r}(x) \sim \frac{1}{(2 \pi r)^{1 / 2}}\left(\frac{e x}{2 r}\right)^{r},(-)^{r} K_{r}(x) \sim(-)^{r}\left(\frac{\pi}{2 r}\right)^{1 / 2}\left(\frac{e x}{2 r}\right)^{-r}$

which are valid for fixed $\mathrm{x}$ and large $r$, we obtain

$$
J_{r}\left(x_{1}\right)<I_{r}\left(x_{2}\right)<(-){ }^{r_{r}}\left(x_{2}\right)<Y_{r}\left(x_{1}\right)
$$

when $x_{1}<x_{2}$ and

$$
I_{r}\left(x_{2}\right)<J_{r}\left(x_{1}\right)<Y_{r}\left(x_{1}\right)<(-){ }^{K_{r}}\left(x_{2}\right)
$$

when $x_{2}<x_{1}$, where in (4.1.3) and (4.1.4) the symbol < is used to indicate the order of dominance of the functions as $r \rightarrow \infty$. Thus when $x_{1} \neq x_{2}$ the JYIK operator is totally separable. As with any totally separable operator of order 4, there are four distinct types of solution and the dimension of the subdominant subspace of type $s$ is $s$. This means that $j=s$ is the proper number of initial values to specify for a solution of type $s$.

When all four Bessel functions have the same argument, from (4.1.1)

and $(4.1 .2)$ we have

$(4.1 .5) \quad J_{r}(x) \sim I_{r}(x),(-)^{r} K_{r}(x) \sim(-)^{r+1} \frac{1}{2} \pi Y_{r}(x), \quad r \rightarrow \infty$.

The first of these relations suggests that we define the further solution 


$$
A_{r}(x)=J_{r}(x)-I_{r}(x)
$$

Then it may be verified that

$$
A_{r}(x) \sim-\frac{1}{2} \frac{x^{2}}{r+1}\left\{\begin{array}{c}
I_{r}(x) \\
J_{r}(x)
\end{array}\right\}, \quad r \rightarrow \infty
$$

where either function in the braces on the right may be chosen. Furthermore, since there is no nonzero linear combination of $(-){ }^{r} K_{r}(x)$ and $Y_{Y}(x)$ that reduces to a solution of lower type, we conclude that there are exactly three distinct types of solution. Symbolically, we have

$$
A_{r}(x)<\left\{\begin{array}{c}
I_{r}(x) \\
J_{r}(x)
\end{array}\right\}<\left\{\begin{array}{c}
(-)^{r}{ }_{r}(x) \\
\\
Y_{r}(x)
\end{array}\right\}
$$

where in each pair of braces either function may be chosen. Thus when $x_{1}=x_{2}$ the JYIK operator is separable but not totally separable. The dimensions of the subdominant subspaces of types 1,2 and 3 are 1,2 and 4 respectively.

Although $A_{r}(x)$ has type 1 , it is separated only by a relative factor of $1 / r$ from solutions of type 2. Let $y_{n}(r)$ denote the approximation to $A_{r}(x)$ that is obtained from the algorithm with terminal point $n$. Then, suppressing the arguments of $A_{r}(x)$ and all the Bessel functions and using equation $(3.2 .6)$, we have ${ }^{t}$

$$
y_{n}(r) \sim A_{r}-\beta_{1}(n) I_{r}-\beta_{2}(n) Y_{r}-\beta_{3}(n)(-){ }^{r_{K}}, n \rightarrow \infty
$$

where

Since $I_{r}(x), Y_{r}(x)$ and $K_{r}(x)$ are not normalized as required for the validity of $(3.2 .6)$, we have only the weaker statement given here. 


$$
\begin{aligned}
& \beta_{1}(n)=\left|\begin{array}{ccc}
A_{n} & Y_{n} & (-)^{n_{K}} K_{n} \\
A_{n+1} & Y_{n+1} & (-)^{n+I_{K}} \\
A_{n+2} & Y_{n+2} & (-)^{n+2} K_{n+2}
\end{array}\right|\left|\begin{array}{ccc}
I_{n} & Y_{n} & (-)^{n} K_{n} \\
I_{n+1} & Y_{n+1} & (-)^{n+1} K_{n+1} \\
I_{n+2} & Y_{n+2} & (-)^{n+2} K_{n+2}
\end{array}\right|, \\
& \beta_{2}(n)=\left|\begin{array}{lll}
I_{n} & A_{n} & (-)^{n_{n}} K_{n} \\
I_{n+1} & A_{n+1} & (-)^{n+I_{n+1}} \\
I_{n+2} & A_{n+2} & (-)^{n+2} K_{n+2}
\end{array}\right||| \begin{array}{ccc}
I_{n} & Y_{n} & (-)^{n} K_{n} \\
I_{n+2} & Y_{n+1} & (-)^{n+I_{K}} \\
I_{n+2} & Y_{n+2} & (-)^{n+2} K_{n+2}
\end{array} \mid,
\end{aligned}
$$

and

$$
\beta_{3}(n)=\left|\begin{array}{lll}
I_{n} & Y_{n} & A_{n} \\
I_{n+1} & Y_{n+1} & A_{n+1} \\
I_{n+2} & Y_{n+2} & A_{n+2}
\end{array}\right|\left|\begin{array}{lll}
I_{n} & Y_{n} & (-)^{n_{n}} \\
I_{n+1} & Y_{n+1} & (-)^{n+1} K_{n+1} \\
I_{n+2} & Y_{n+2} & (-)^{n+2} K_{n+2}
\end{array}\right| .
$$

In consequence of $(4.1 .1),(4.1 .2)$, and $(4.1 .7)$ we see that $\beta_{1}(n),-x^{2}(2 n)^{-1}$ for large $n$, whereas $\beta_{2}(n)$ and $\beta_{3}(n)$ approach zero much more rapidly. Thus we expect that the rate of convergence of the algorithm will be approximately as $1 / n$. This has been confirmed in numerical tests. Since the separation of $A_{r}(x)$ from solutions of type two is so weak, it is usually advantageous to regard $\mathrm{A}_{\mathrm{r}}(\mathrm{x})$ as belonging to the same type as $J_{r}(x)$ and $I_{r}(x)$; this means that we specify two initial conditions rather than one. This modification improves convergence; in consequence the value of the terminal point $n$ is reduced very considerably. This gain is offset to a minor extent by some loss in accuracy in the values of $A_{r}(x)$ for higher values of $r$; the extent of this loss is no more than the cancellation that takes place between the corresponding values of $I_{r}(x)$ and $J_{r}(x)$ in Eq. $(4.1,6)$. 
Exampie 4.1.1 In this example we compute the solutions $\mathrm{J}_{\mathrm{r}}(1), \mathrm{I}_{\mathrm{r}}(10)$, $(-)^{r_{K}}(10)$ and $Y_{r}(1)$ of the homogeneous equation defined in Figure 6 , with $x_{1}=1, x_{2}=10$, for $r=0,1,2, \ldots, 100$. Since each of these solutions is zonctonic (in magnitude), the algorithm will be numerically stable provided that the proper number $j$ of initial values is used in each case. This number is $j=1$ for $J_{r}(1), j=2$ for $I_{r}(10)$, $j=3$ for $(-)^{{ }^{T}} \mathrm{~K}_{\mathrm{r}}(10)$ and $j=4$ for $\mathrm{Y}_{\mathrm{r}}(1) ;$ compare (4.1.3). Iable 1 gives the numerical coefficients of the JYIK operator with $x_{1}=1$ and $x_{2}=10$ for $r=0,1,2, \ldots, 109$. Tables 2,3 and 4 give the numerical coejicients of the $(0, j)$-iactorizations of chis JYIK operator For $j=1,2$, and 3 . (We do not present the $(0,4)$-factorization.) since the $(i, j)$-factorization $D_{j}^{i}=A_{j}^{i} B^{i+j}$ produced by the test program has the principal diagonal of $A_{j}^{i}$ equal to $(1,1,1, \ldots)$, we do not include values of $a_{j}(r)$.

Iables 5 through 8 give the results of the computation of the four desired solutions $J_{r}(1), I_{r}(10),(-)^{r_{K}} K_{r}(10)$ and $Y_{r}(1)$ for selected values of $\mathrm{r}$; see 34.0 for a full description of the tables. Initial values were caken from Tables 9.4 and 9.11 in [16]; they are given in parentheses. These tables also supply values of the four Bessel functions for certain other values of $r$ in the range $0 \leq r \leq 100$. These entries all agree with our computed results, except for the tenth significant figure in some instances. This slight discrepancy is explained by the fact that the starting values that were used inevitably have an error of several units in the eleventh significant figure. Incidentally, because in this (and other examples) we have not attempted rigorous control of individual rounding errors, a guaranteed error bound cannot be given. 
89

Nevertheless, the stability of the algorithm for all solutions in this example is firmly demonstrated. 


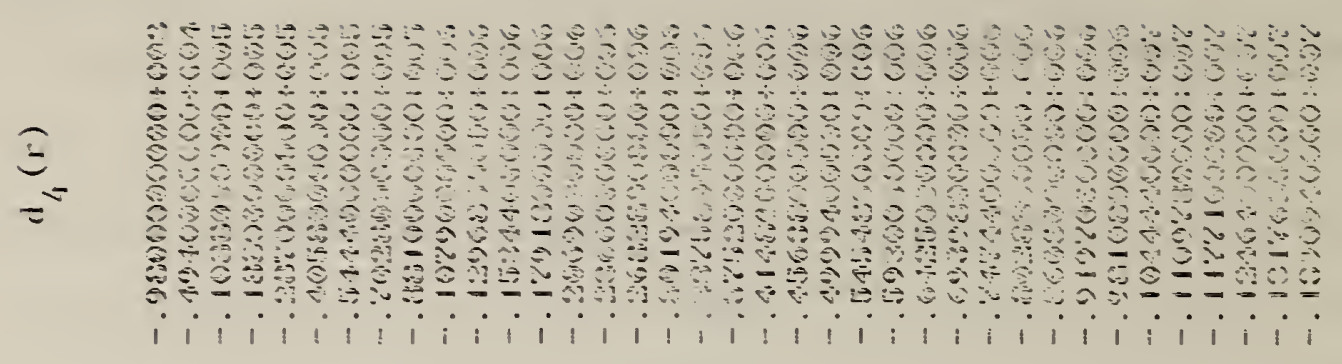

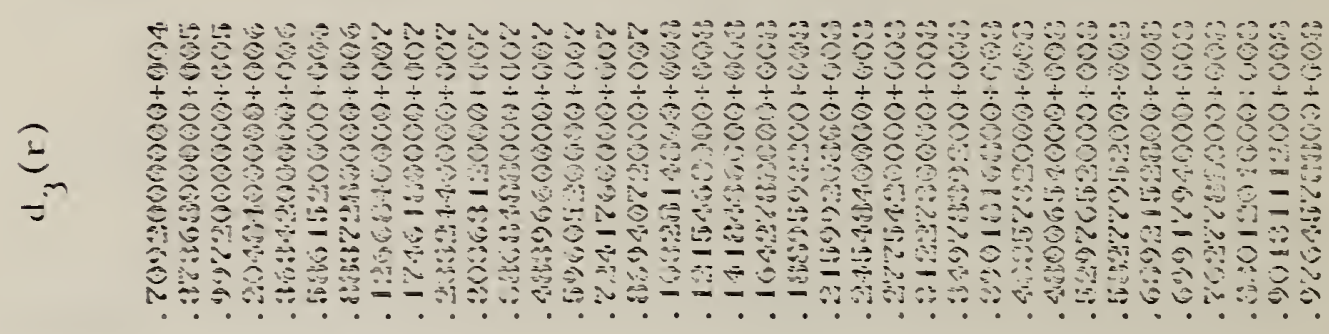

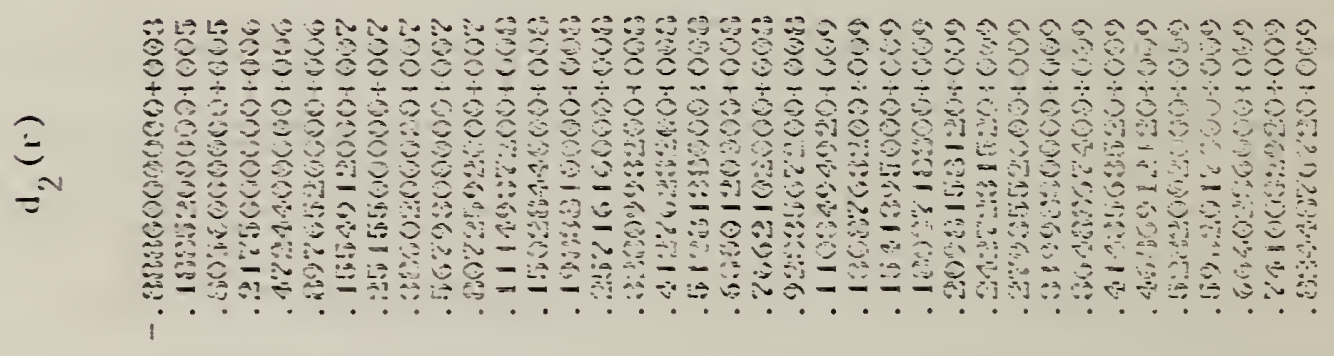

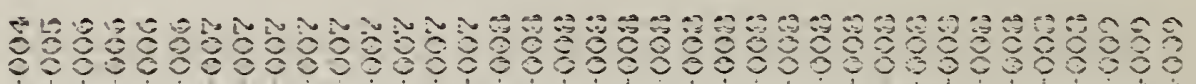
I 七

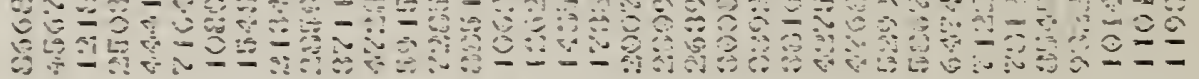

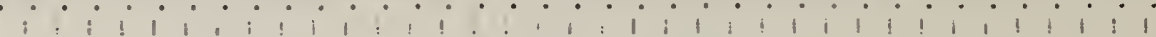

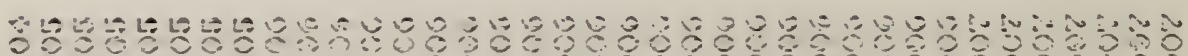
I

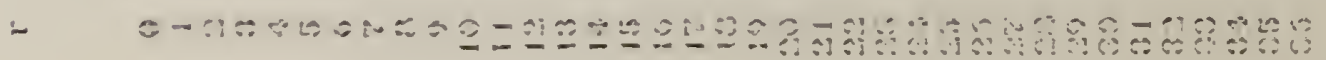




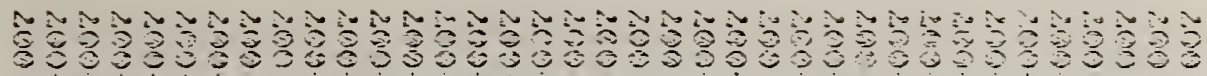

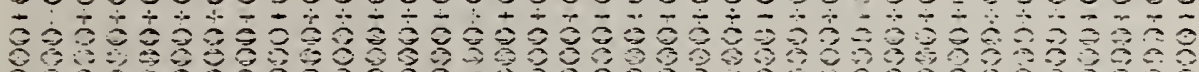

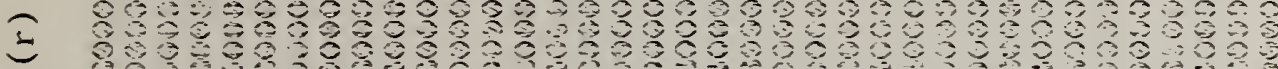
Ј

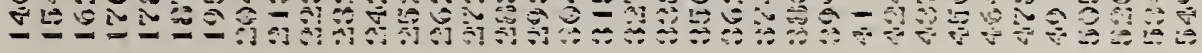

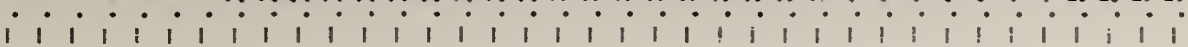

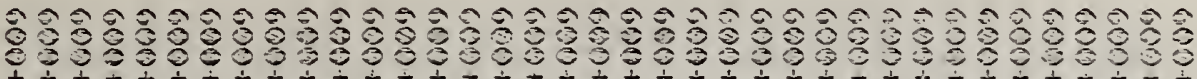

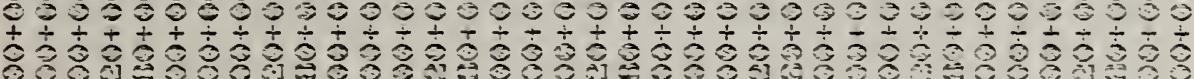

I

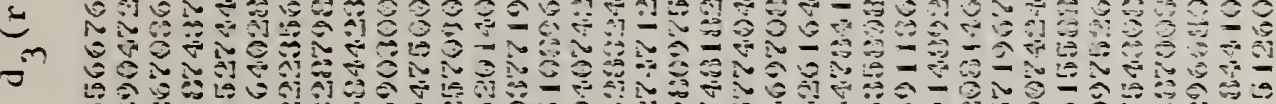

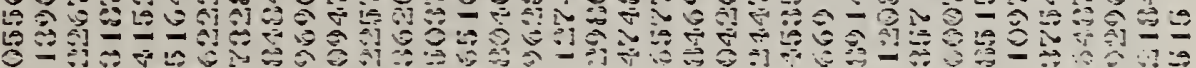

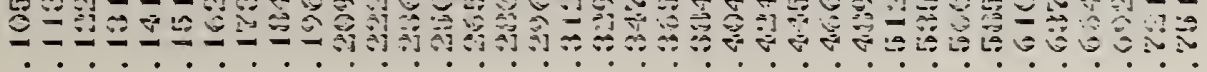

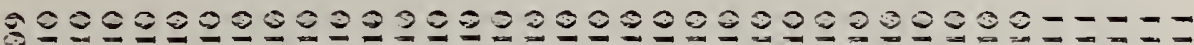

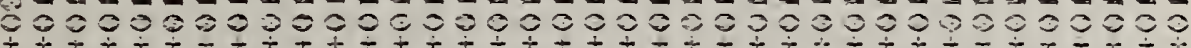

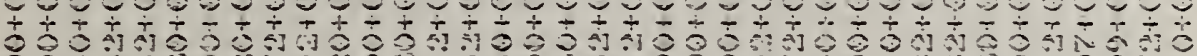

I 00000 。

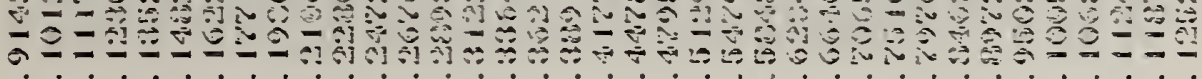

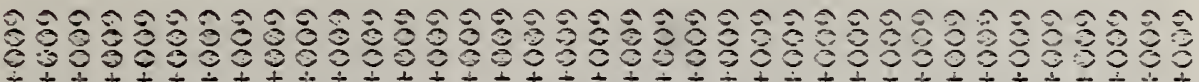

ङ =

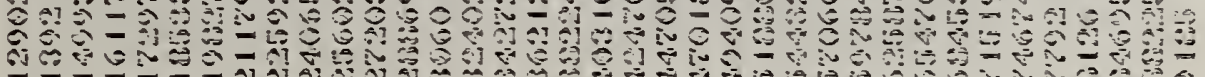
i i i i i i i i i i i i i i i i i i i i i i

N

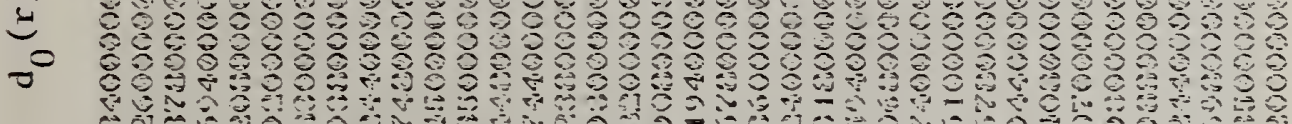

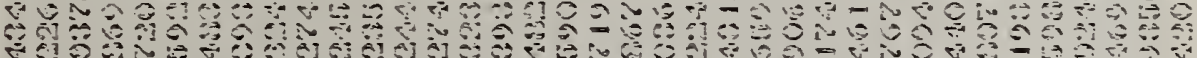

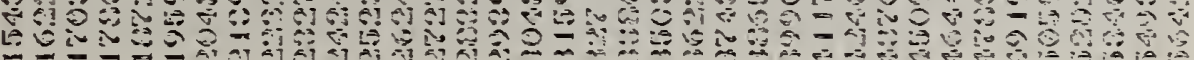




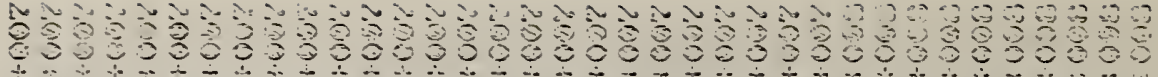

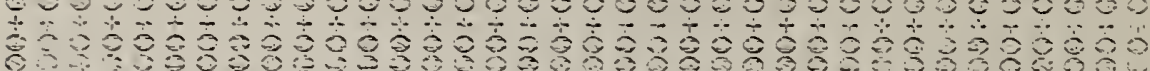
ङ ๑

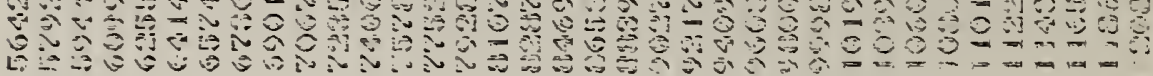
i

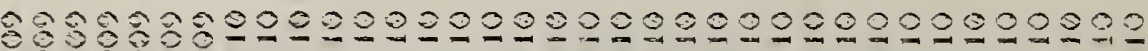
000

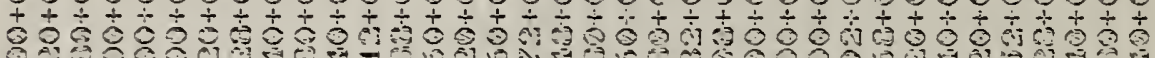

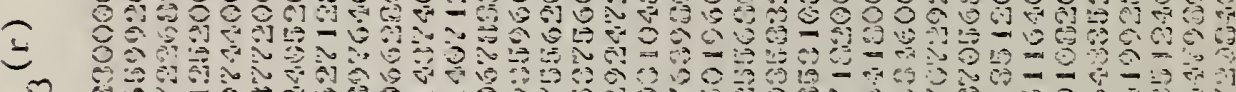

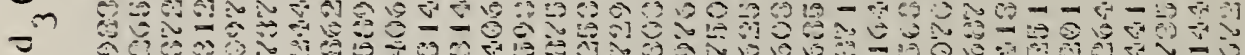

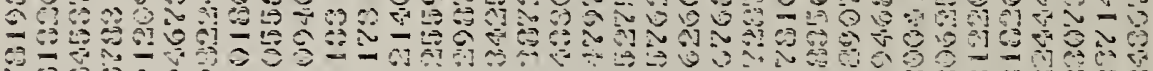

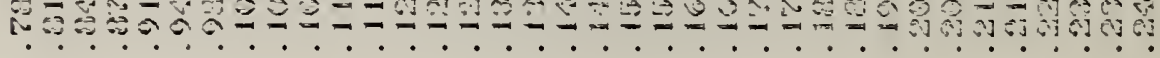

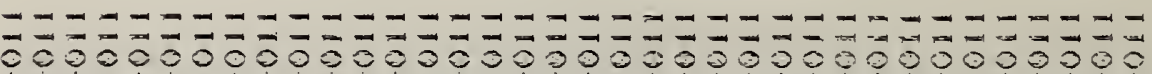

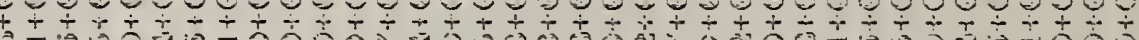
I $\sigma^{2}$ o

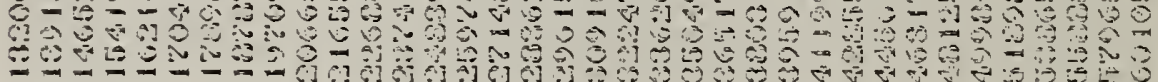

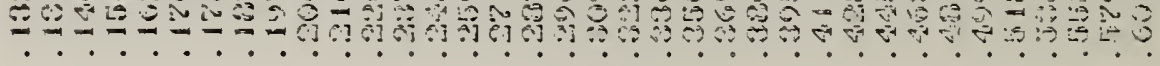

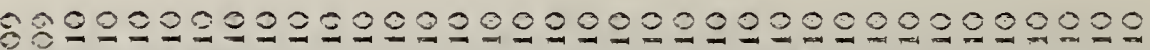
0.000030000000000000030000000000000

Э 830

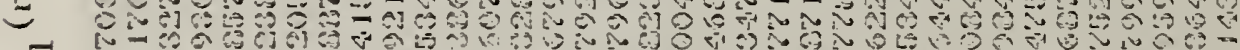

$\checkmark$ 七

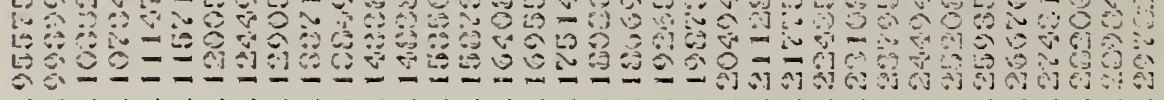
i i i i i i i i i $i i_{i}$ i

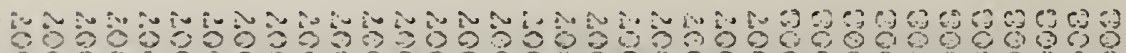

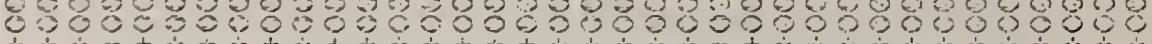
¿ 3030 के 二

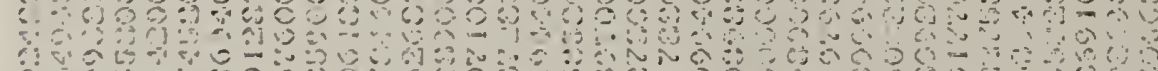
10 $\because \cdots \cdots$

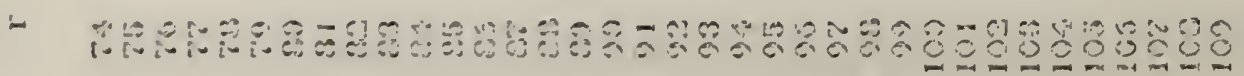




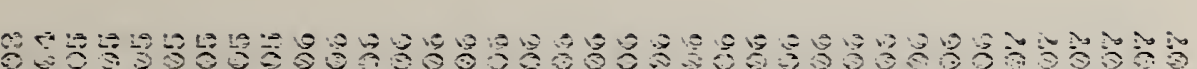

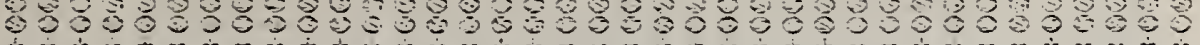
ᄀ

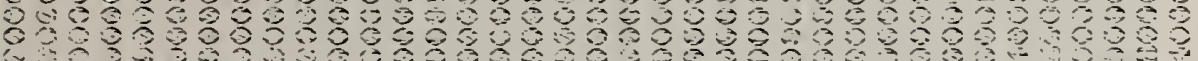

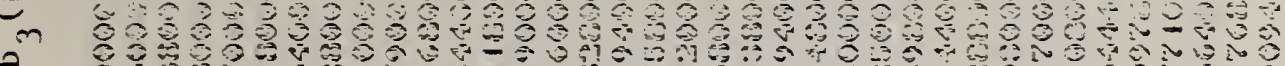

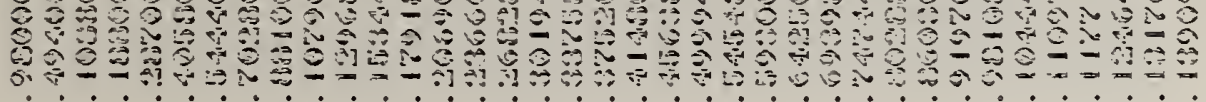
i i i i i i i i i i i i i i i i i i i i i

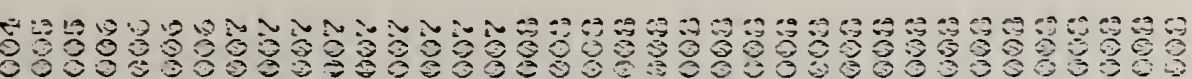

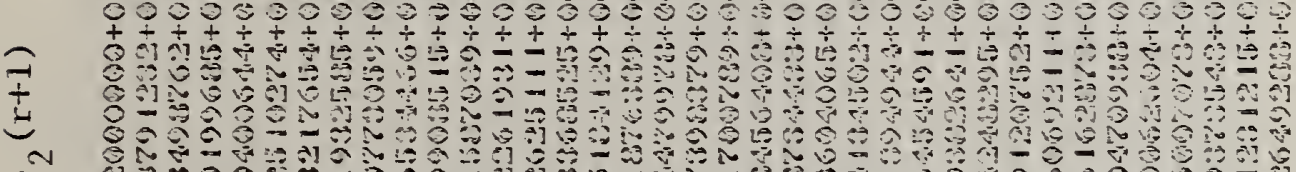

N

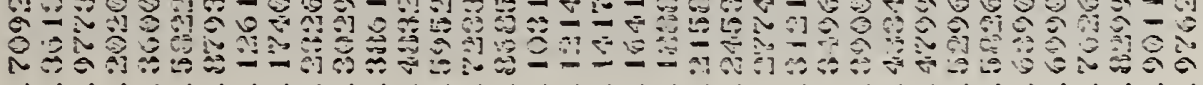

月4

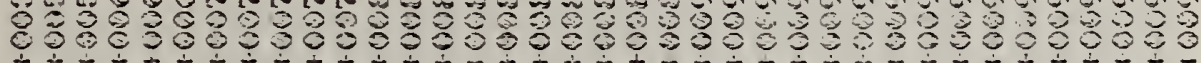

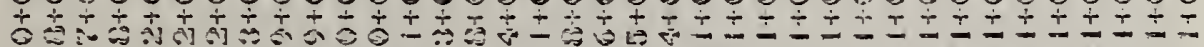

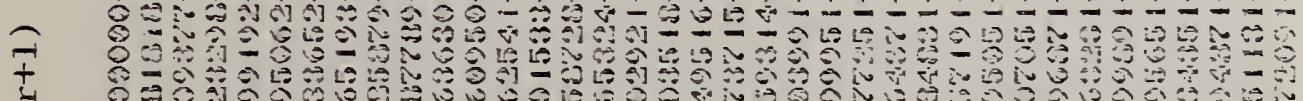

I 8

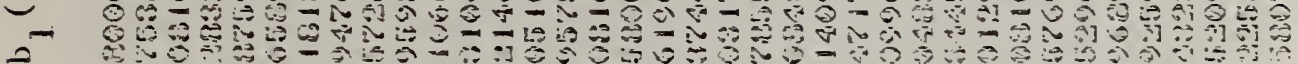

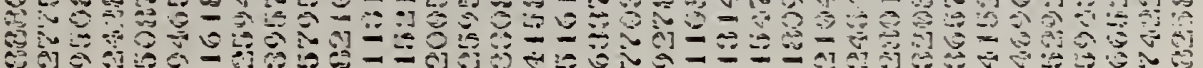

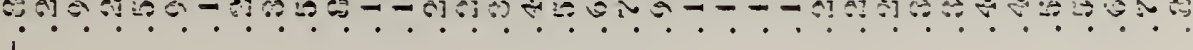

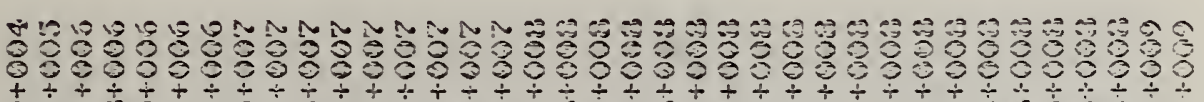

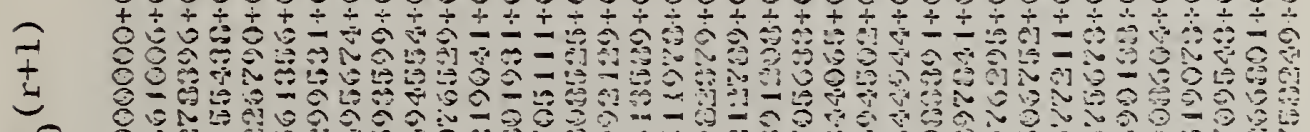

○ 桶

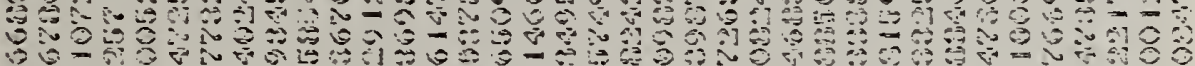

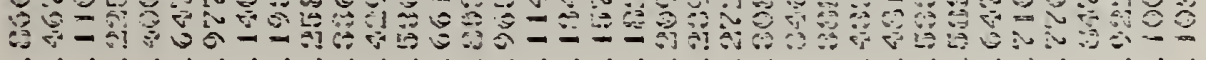

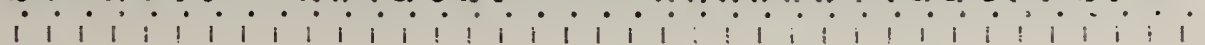

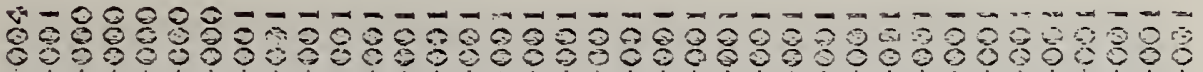

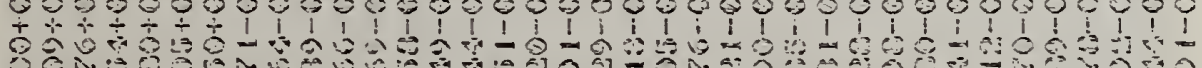

J 3000 80

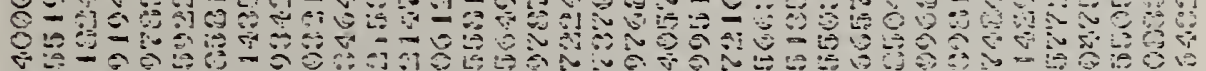

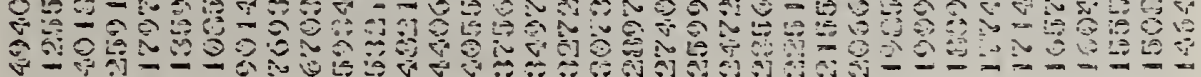
i i i i i i i i i i i i i i i i i i i i i i i

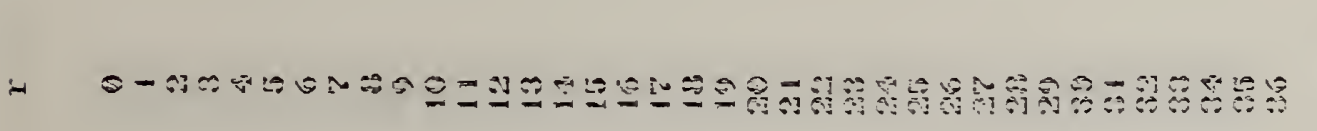




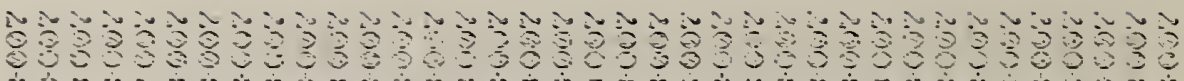
1.

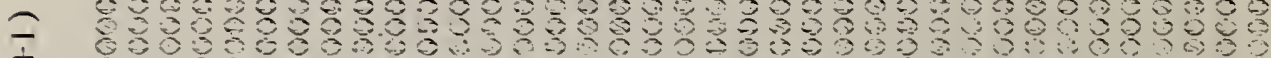

†

a

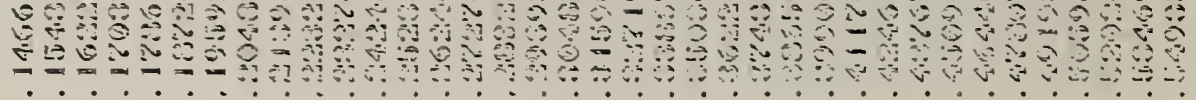
i i i i i i i i i i i i i i i i i i i i i i

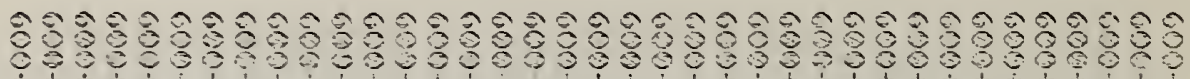

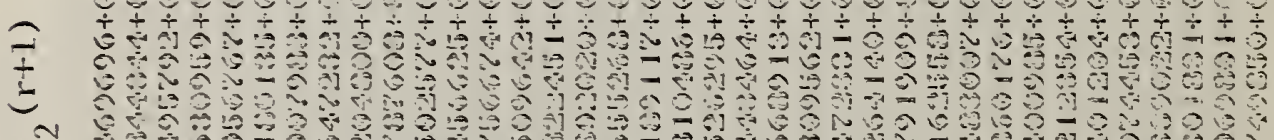
N

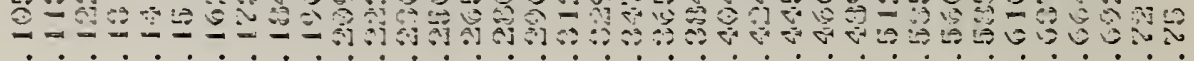

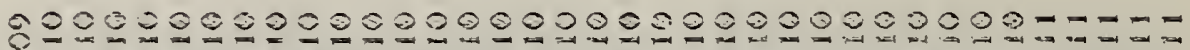

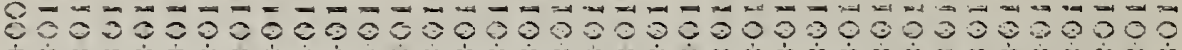

7 t

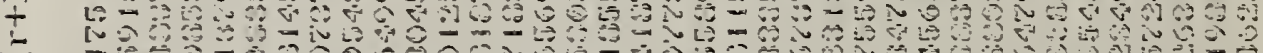

乙

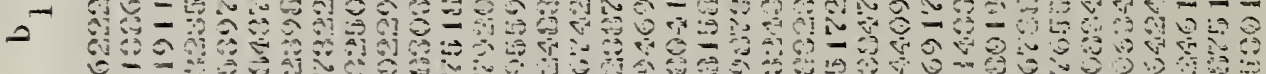
o

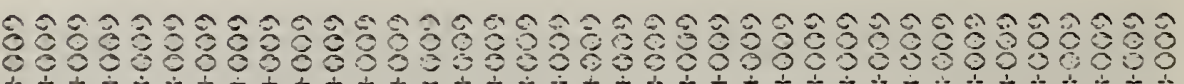
I

I 0 o

J

$=$ a

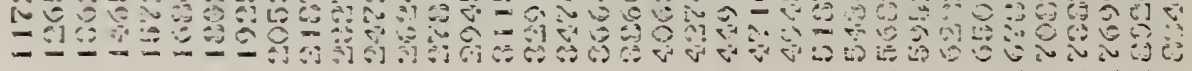
i i i i i i i i i i i i i i i i i i i i ili

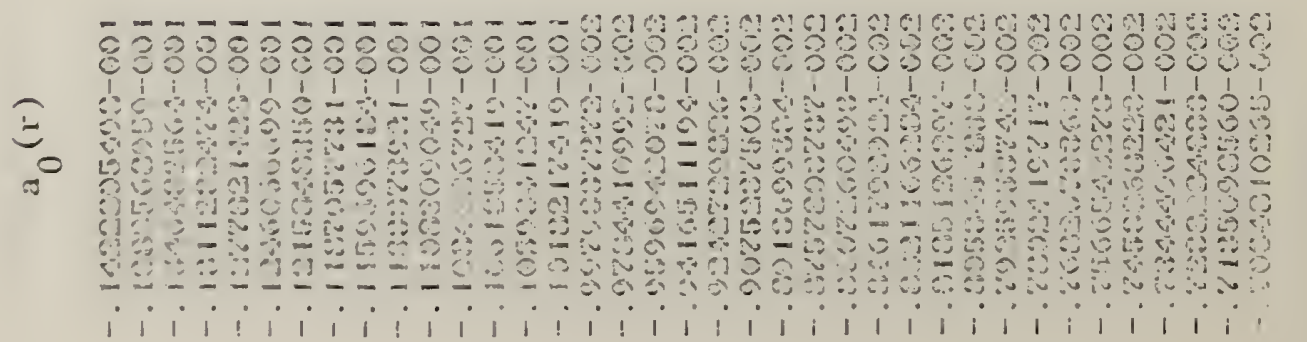

- 


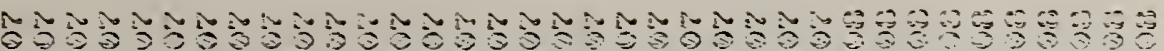

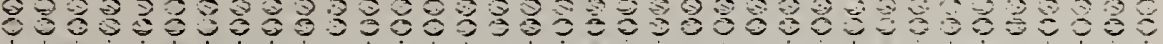
† t

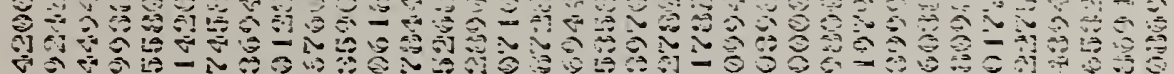

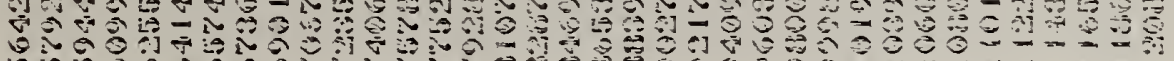

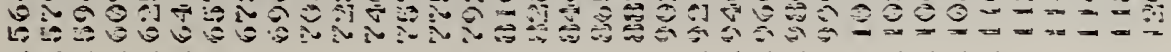

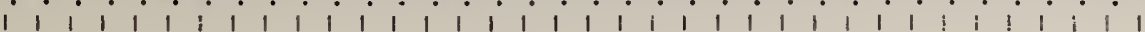

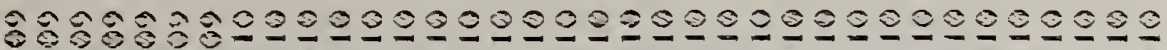

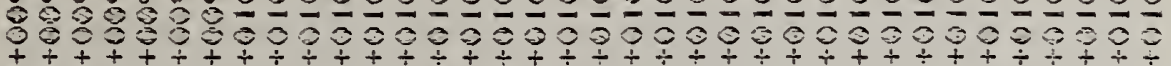

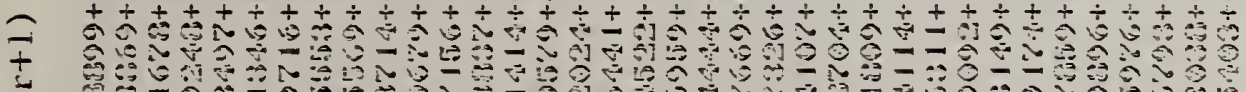

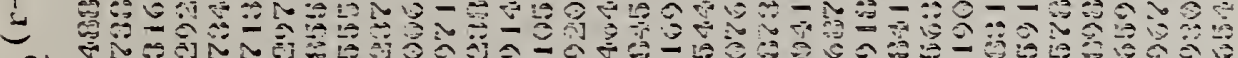

乞

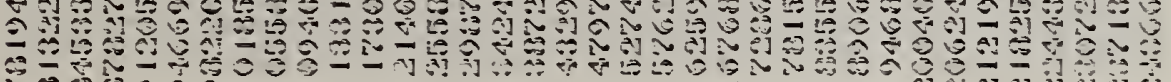

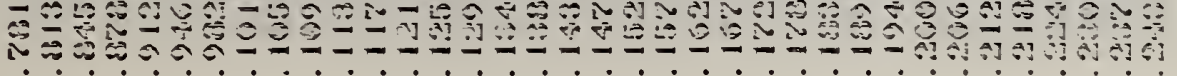

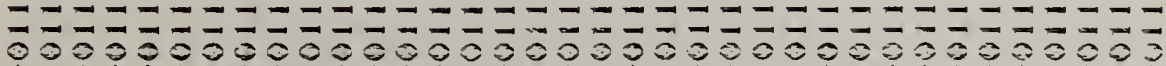

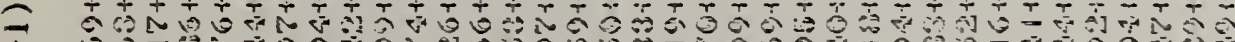

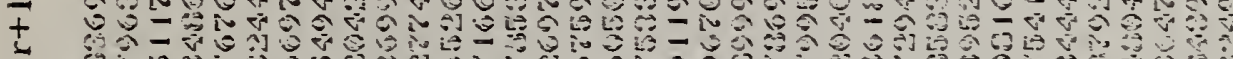

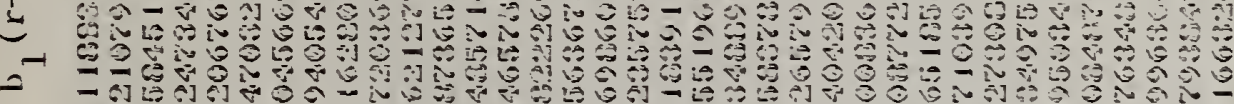

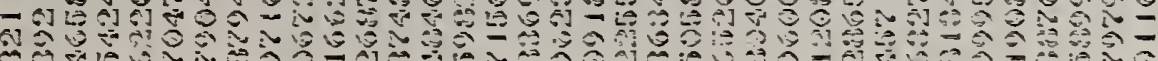

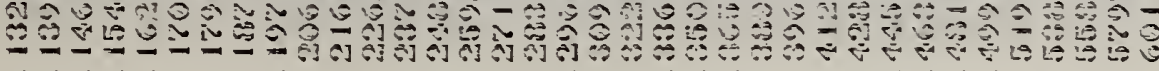

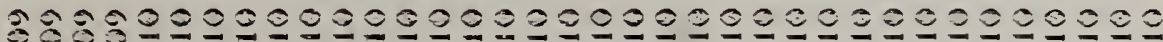
\%

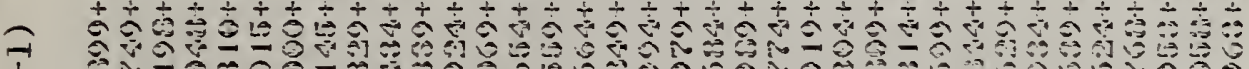

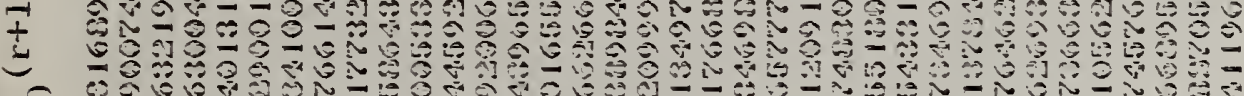
o o

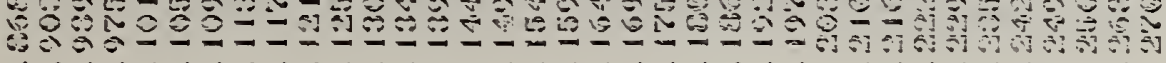

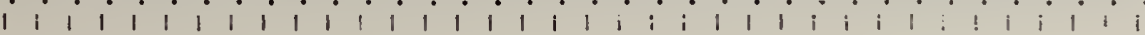

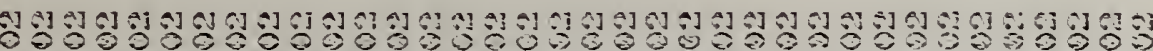

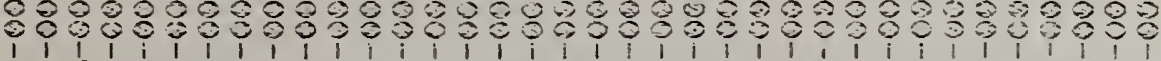

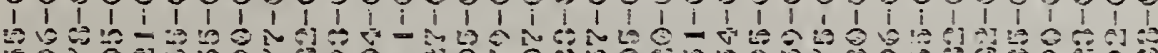

I

\&

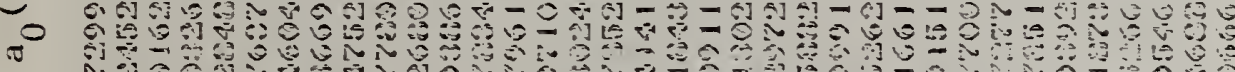

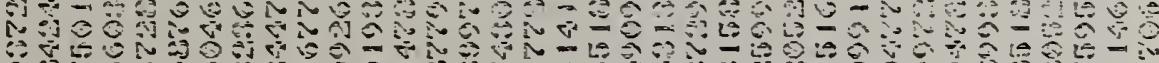

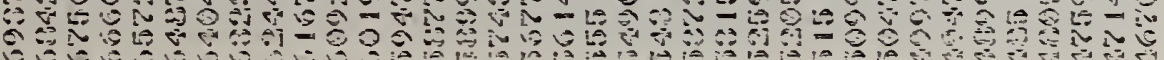

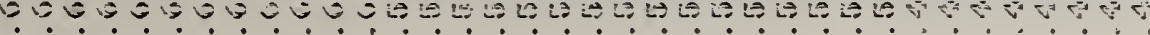
i i i i i i i i i i i i i i i i i i i i

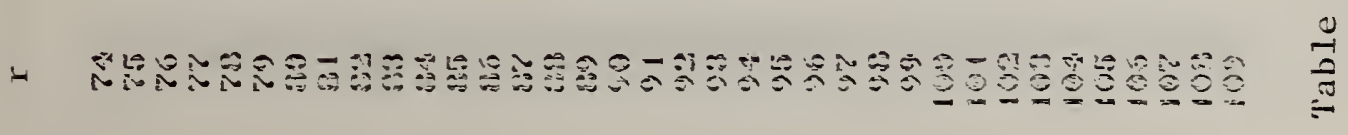




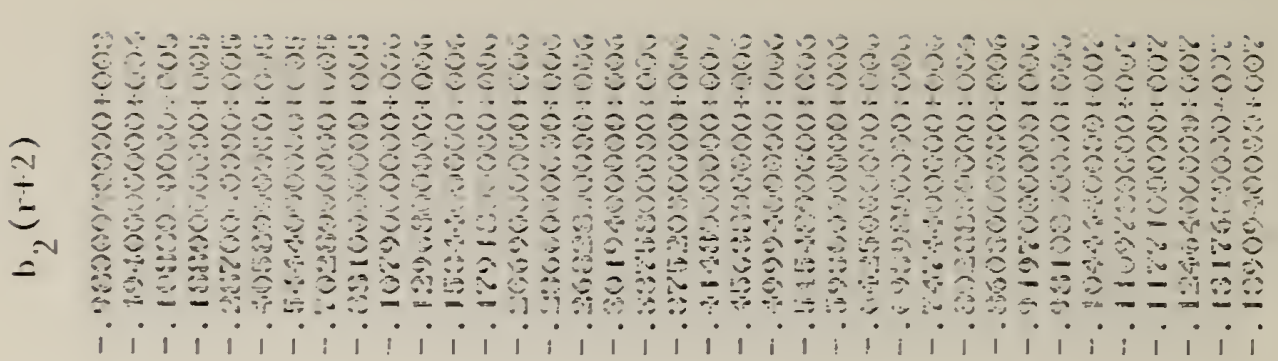

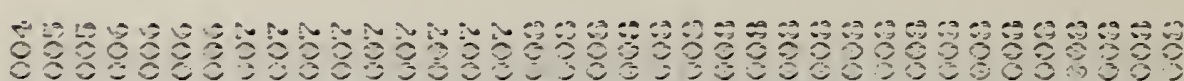

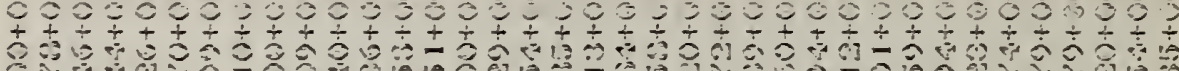
令

† 二

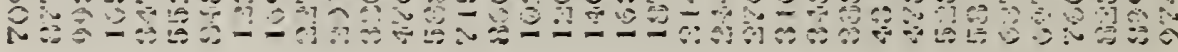

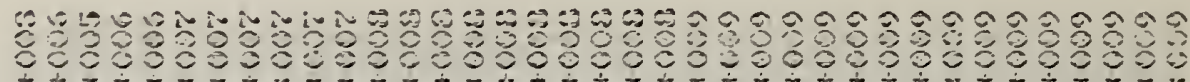

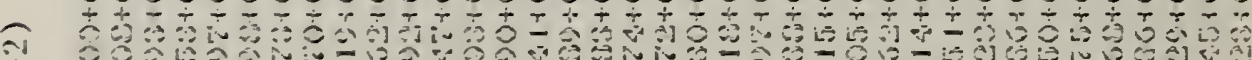

+ o

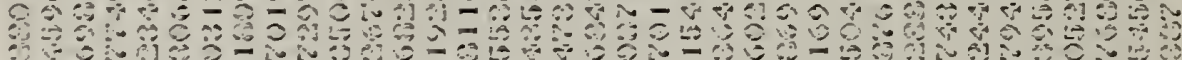

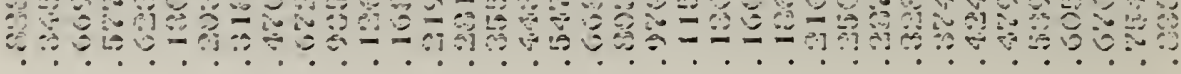
$i i$

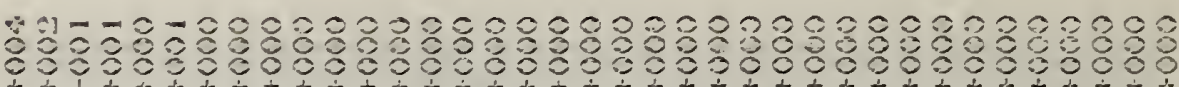

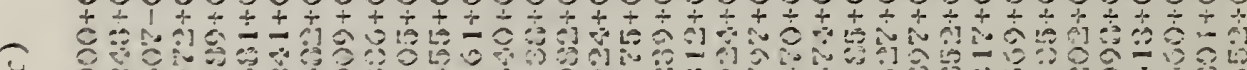

ङ

-

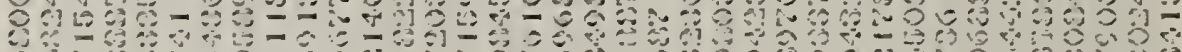

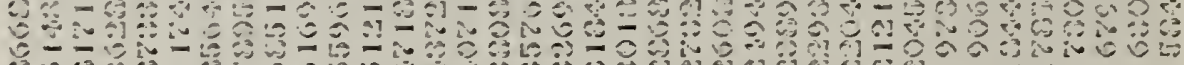

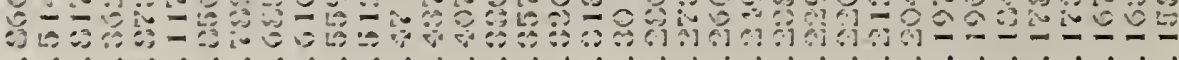
i i i i i i i i i i i i i i i i i i i i

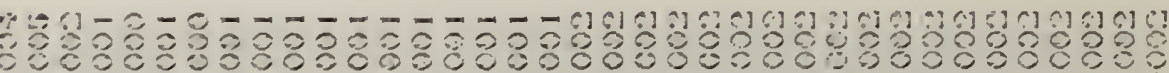

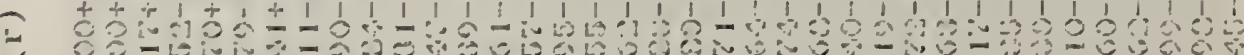

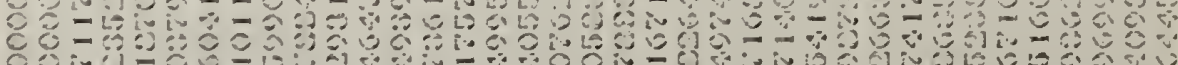
c80

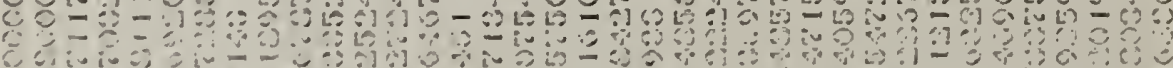

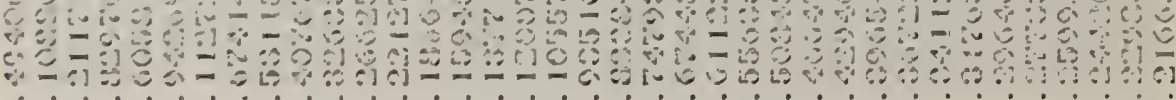

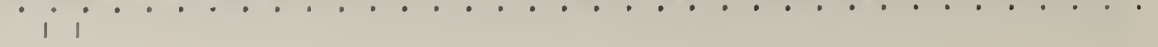

$-1$

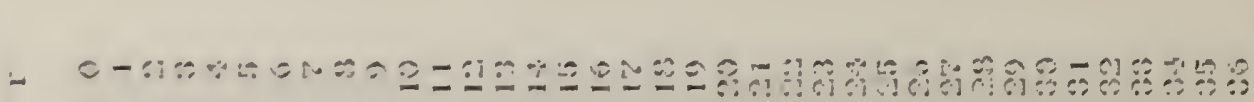




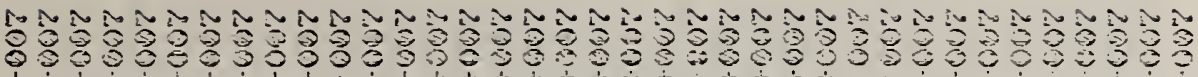
ล

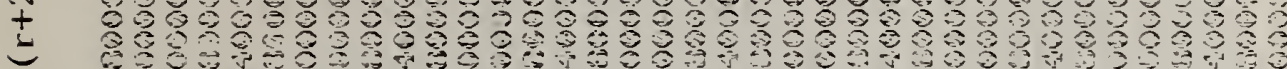
๑ 0

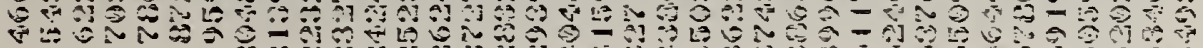

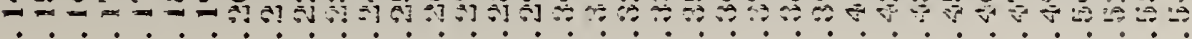

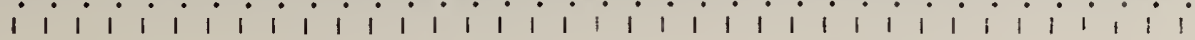

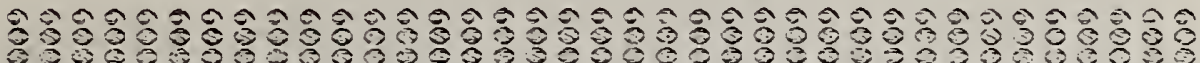
trition

จ

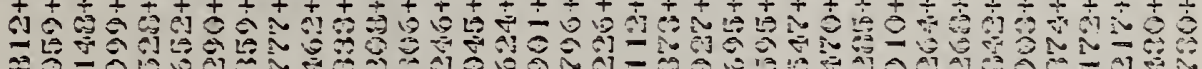

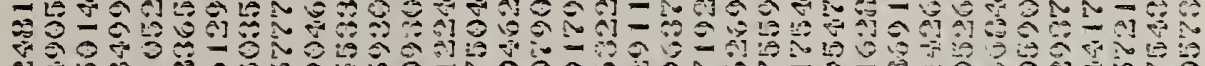
ง

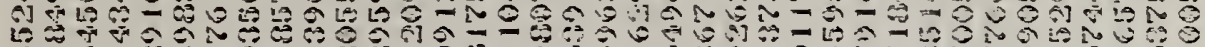
ติ

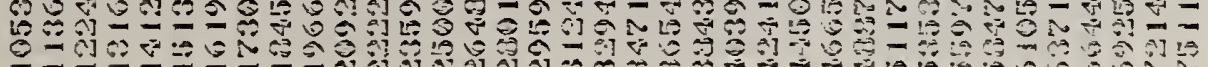

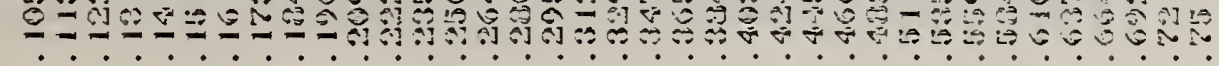

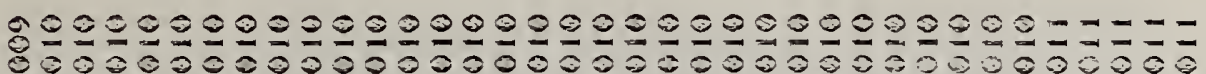

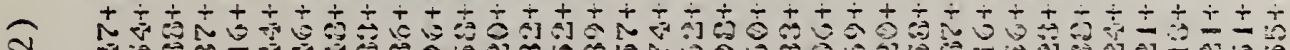

+

I

。

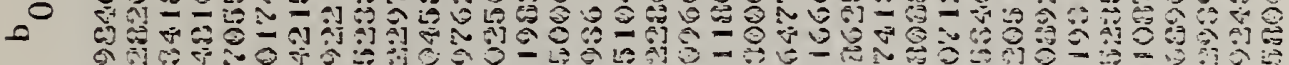

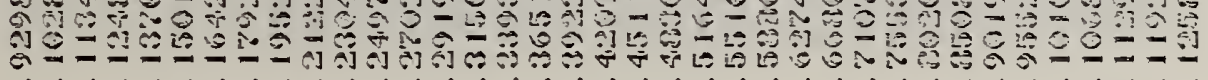

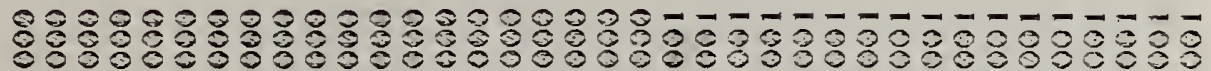
8.

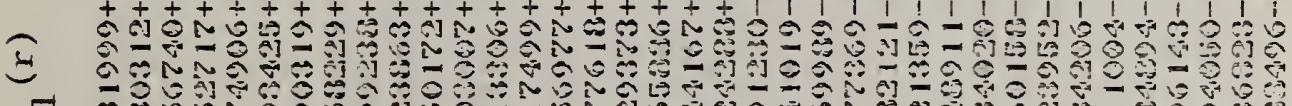

万

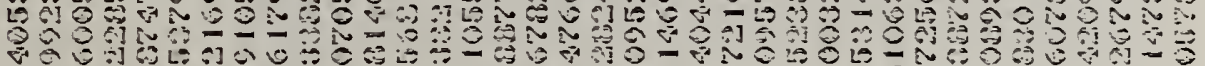

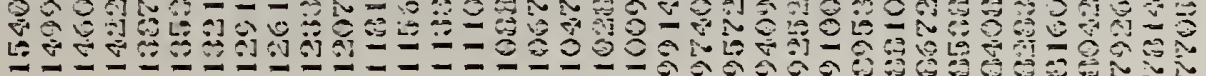

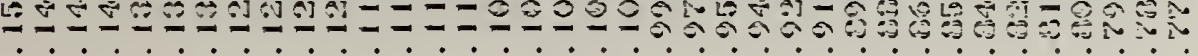

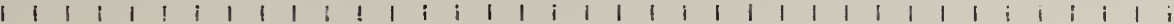

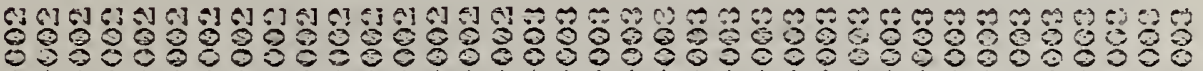

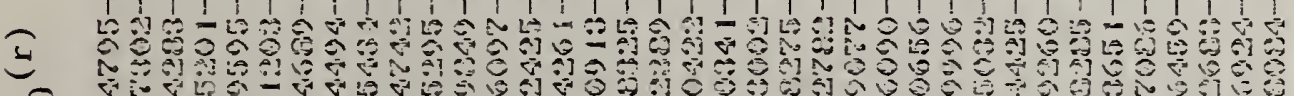

o i

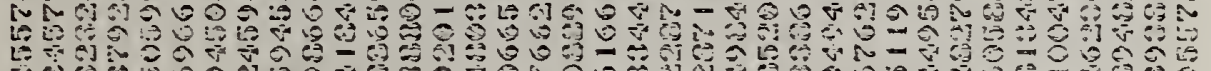

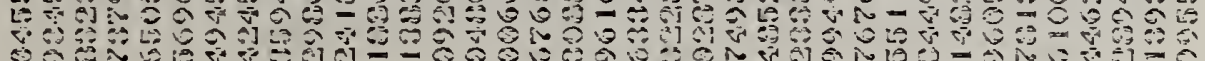

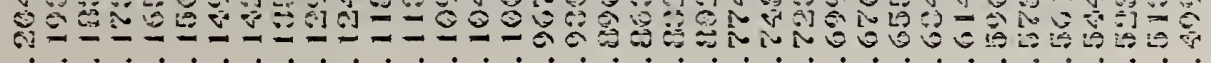

ㄴ.

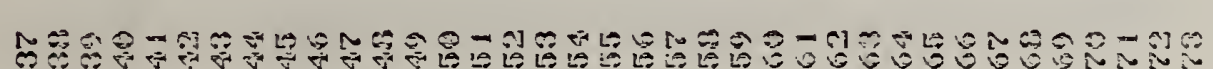




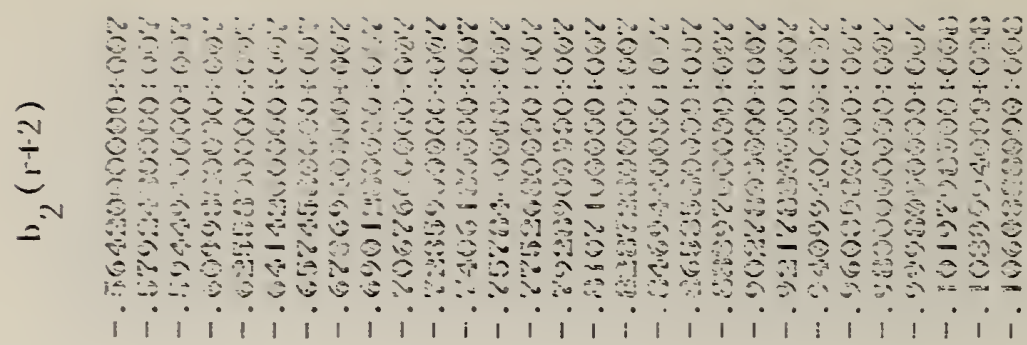

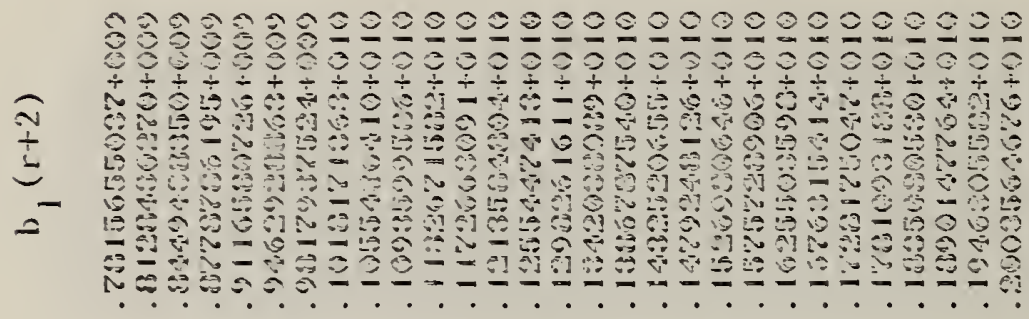

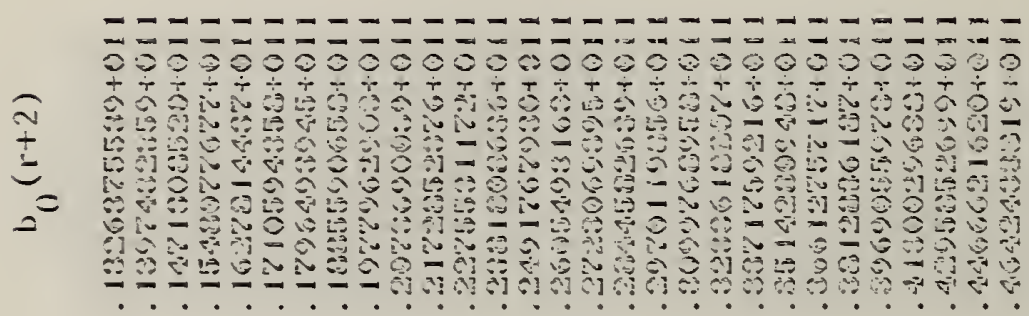

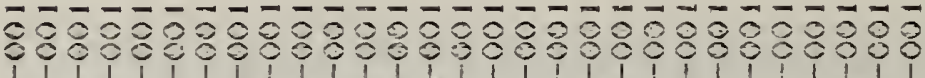

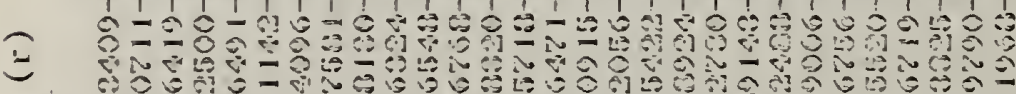

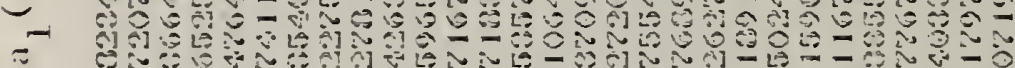

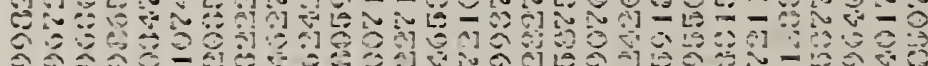
Ẽ.

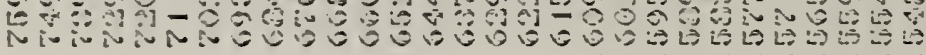

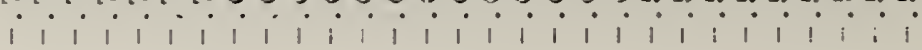

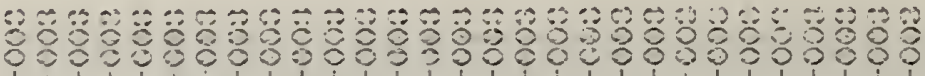

I 100

- 


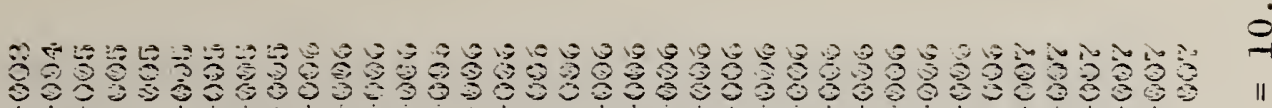

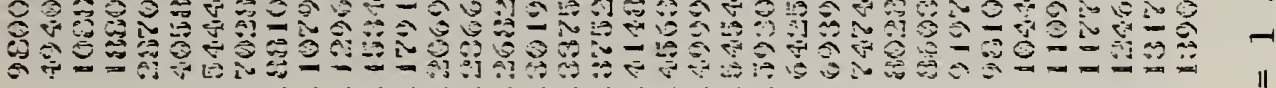

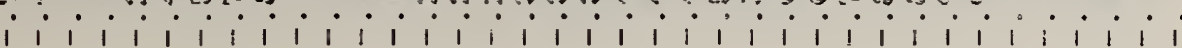

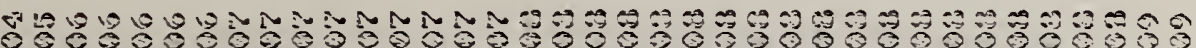
8.8.83\%

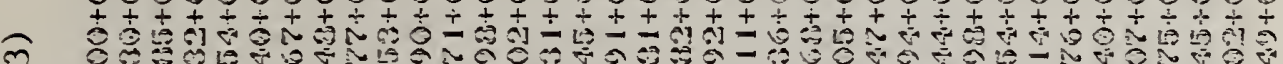

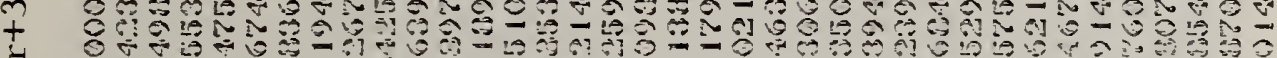

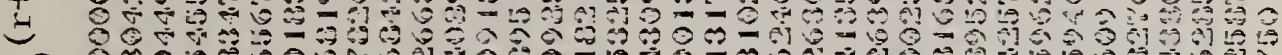

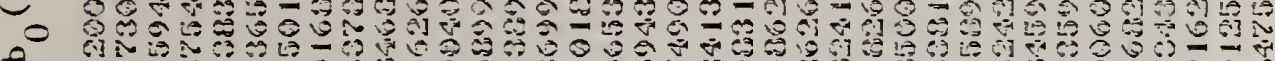

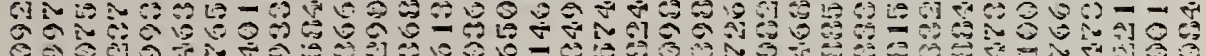

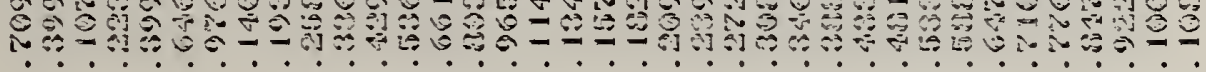

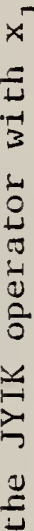

\%

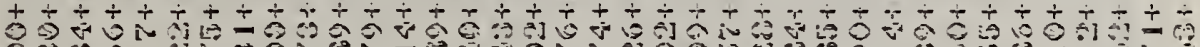

I $\begin{aligned} & 0 \\ & \text { N }\end{aligned}$

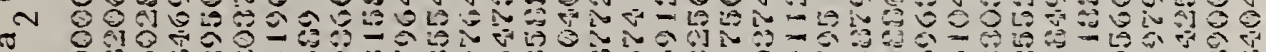

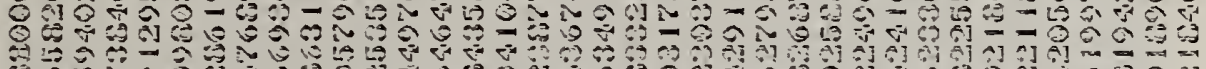

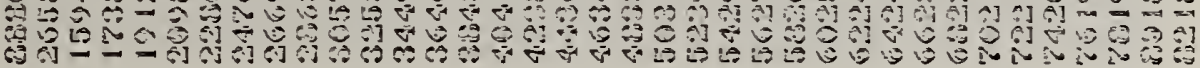

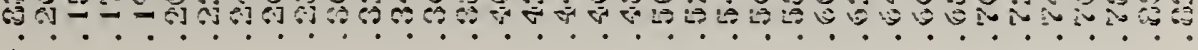

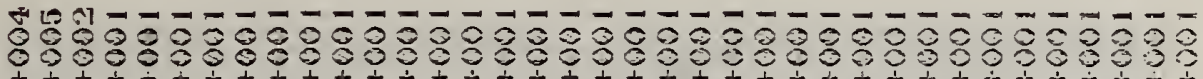

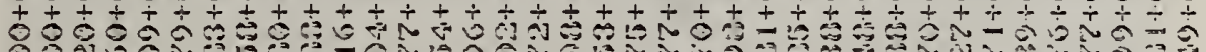

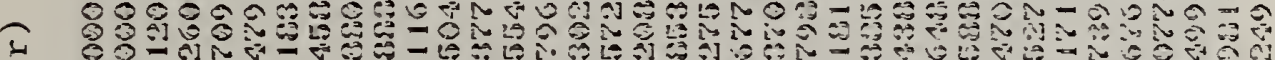

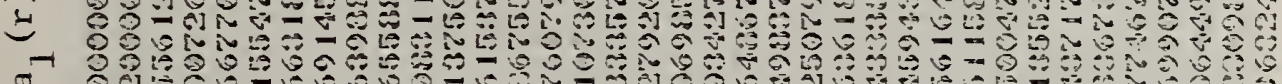
与.

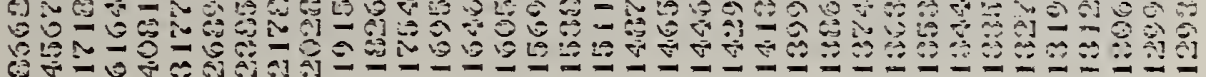

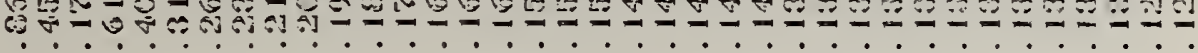

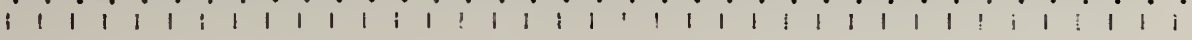

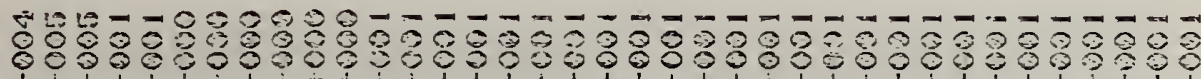

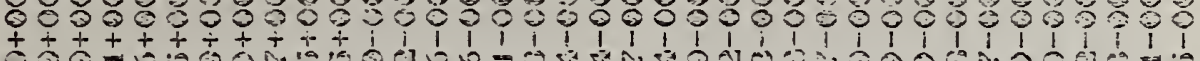

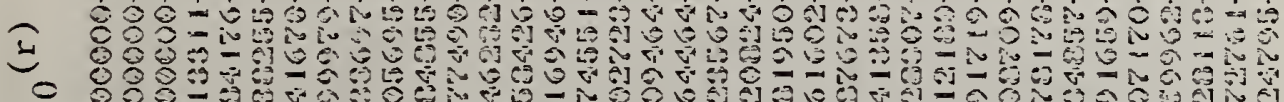
880

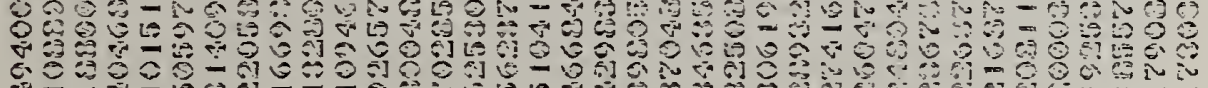

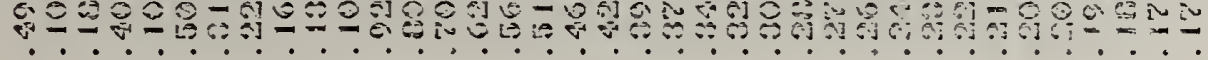

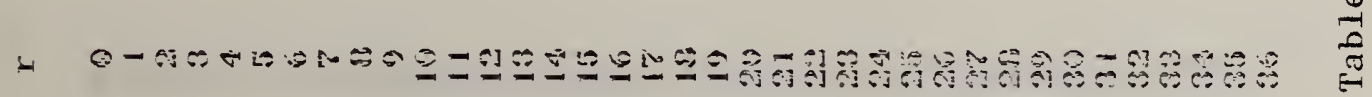




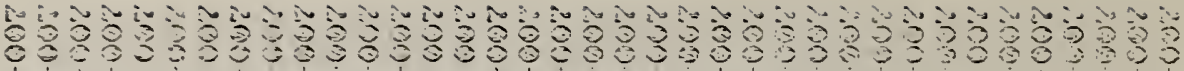

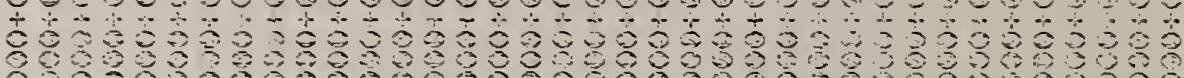
†

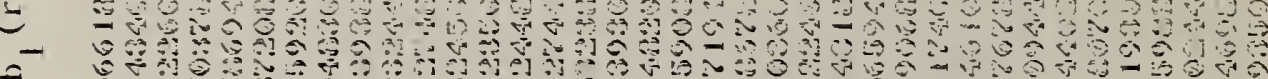

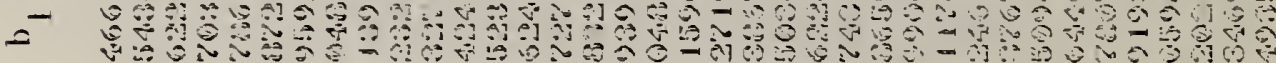

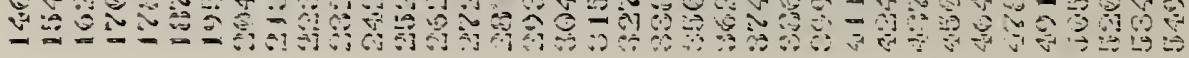
i i i i i i i i i i i i i i i i i i i i i i

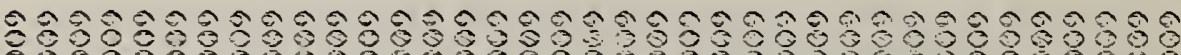

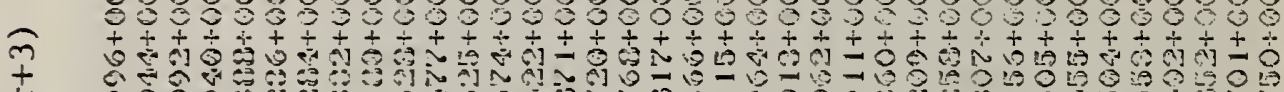

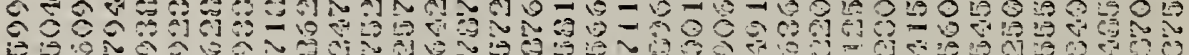
至 to

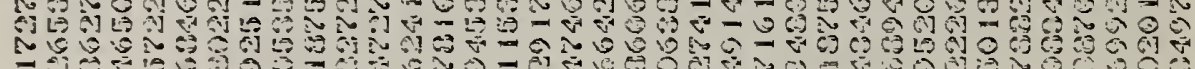

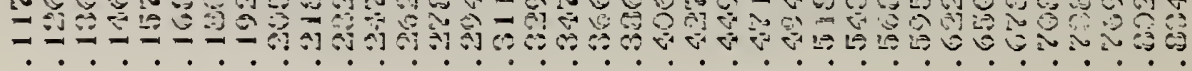

-

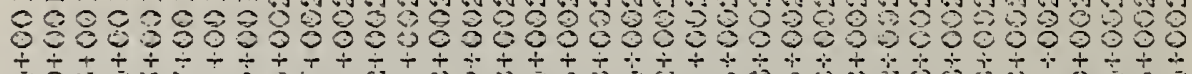

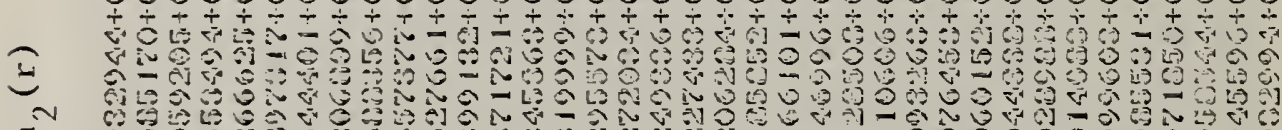

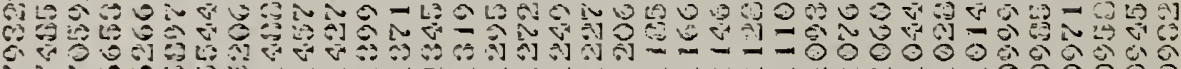
in

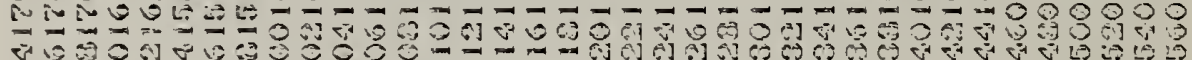

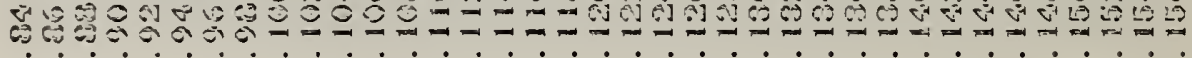

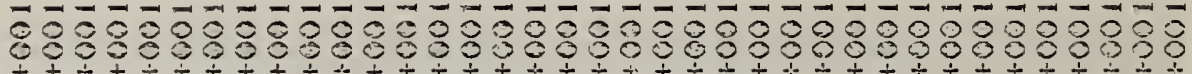

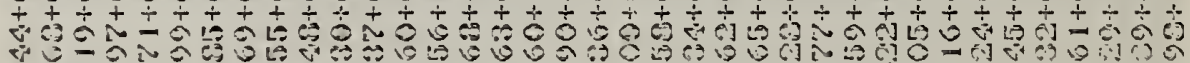
T.

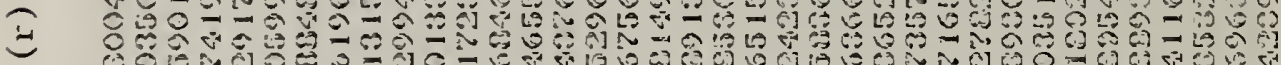
7 6010 v1

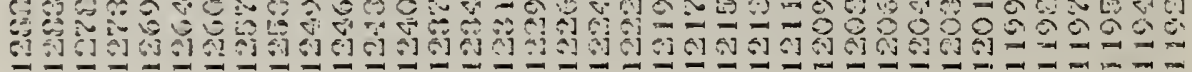

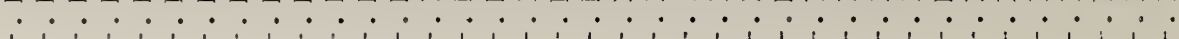

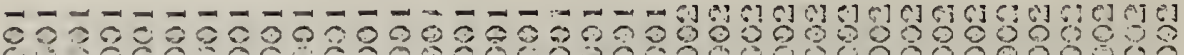
ч I $\quad \begin{aligned} & 1 \\ & 0\end{aligned}$ o d c a 6us

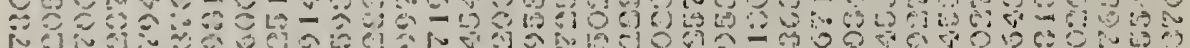

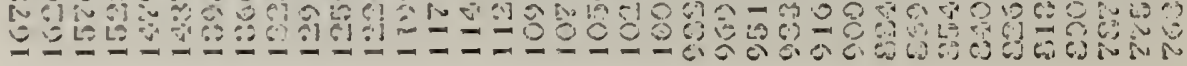

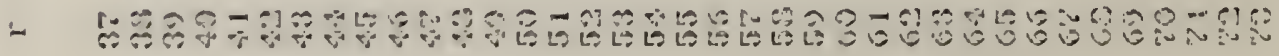




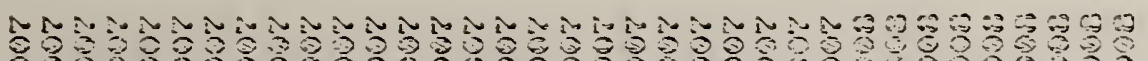
0

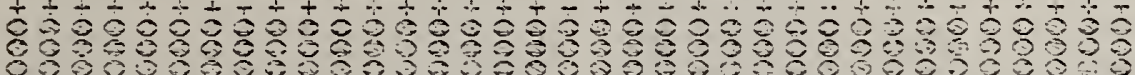

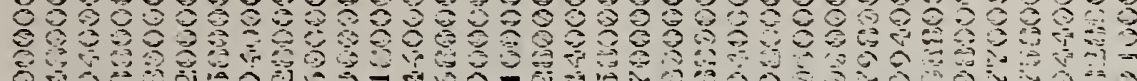
D.

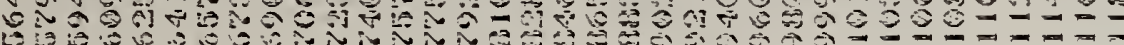

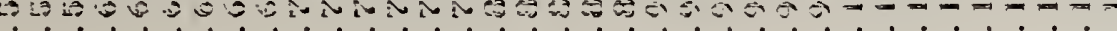
- $i$ i i i i i i i i i i i i i i i i i i

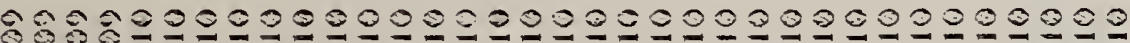
\%

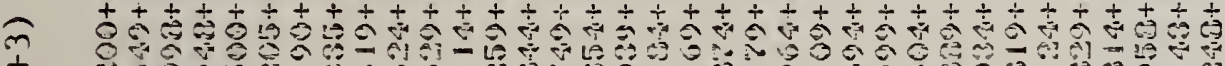
+ t

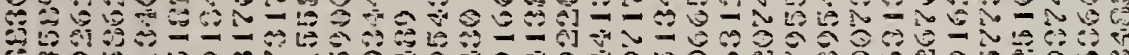

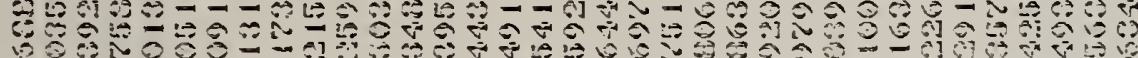

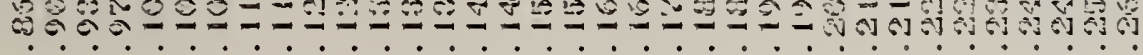

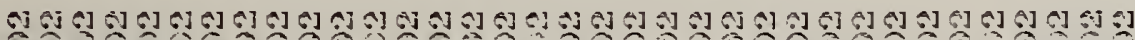

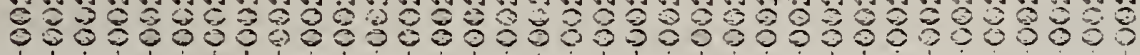

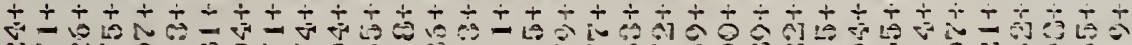

I N

C w

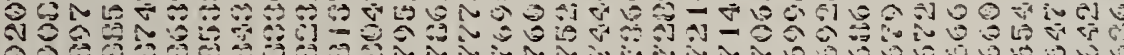

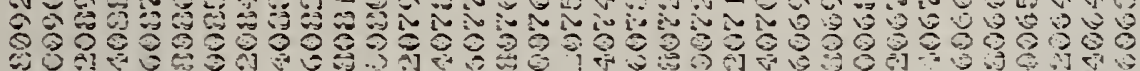

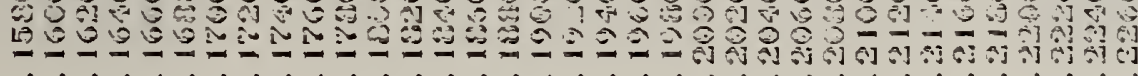

б-б

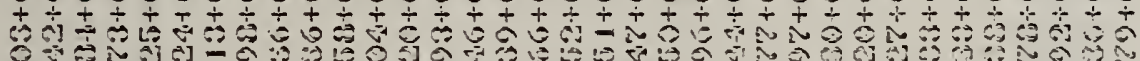

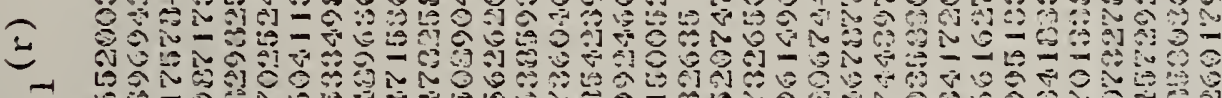

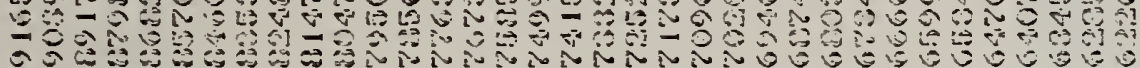

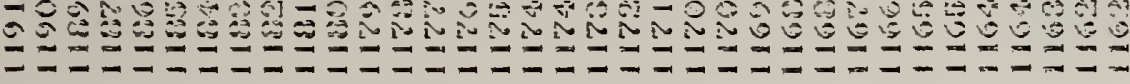
i i i i i i i i i i i i i i i i i i i i i i i

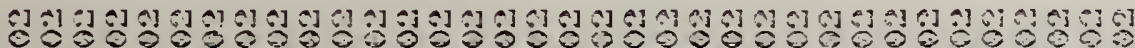

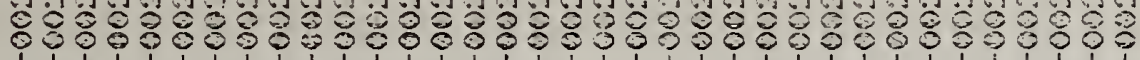

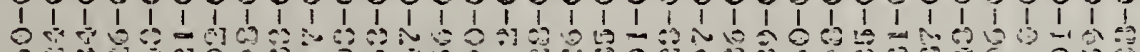

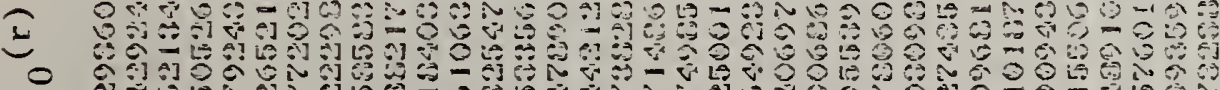

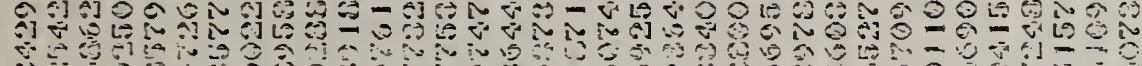
6

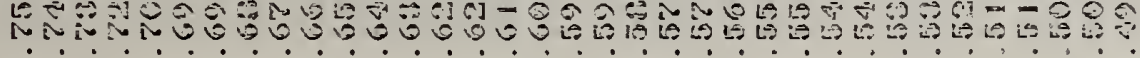

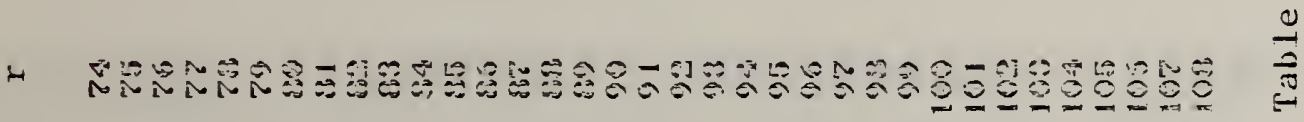



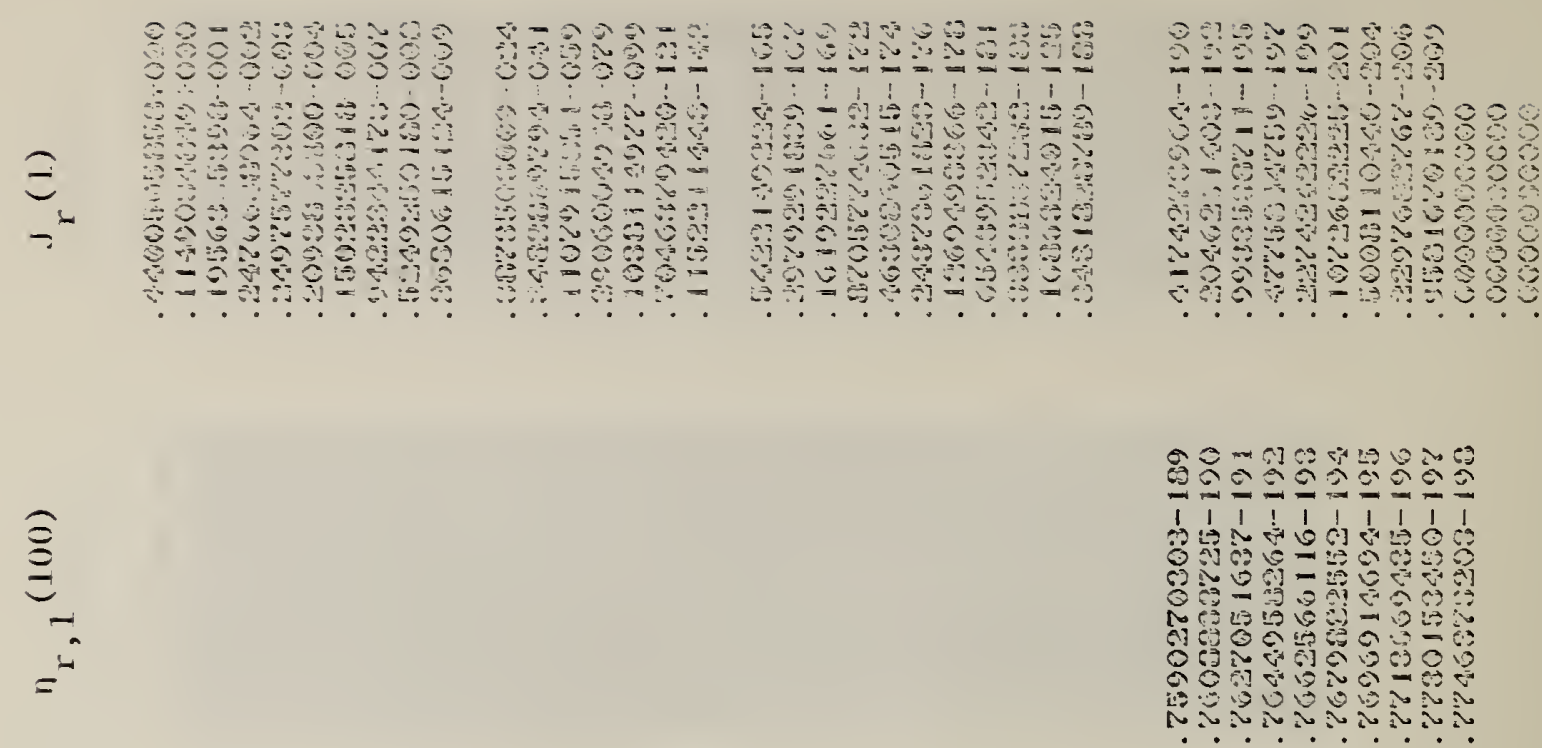

$\underbrace{Ð}_{i}$

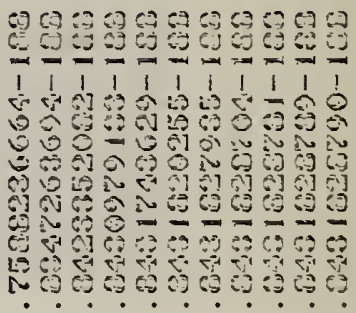

$\stackrel{-i}{\pi}$

\section{$\underset{3}{3}$}

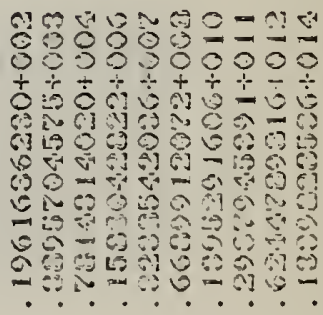

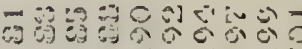

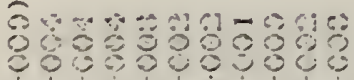
$4+1=t+14$

$\underbrace{2}_{N}$

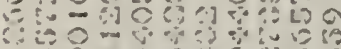
50

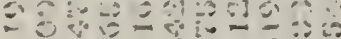
เ9 $\because \frac{0}{2}=0$

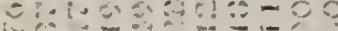
$\because i j i i i j$

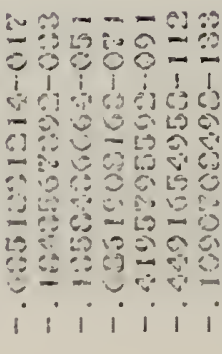

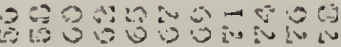

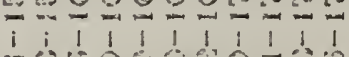

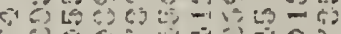

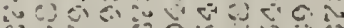
A $\therefore 236-i 01300=0$ cisces

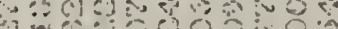

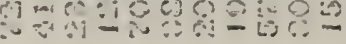
i i i i i i i
$1-11=0$

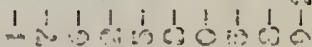

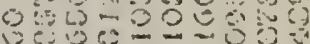

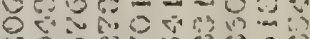

ใग

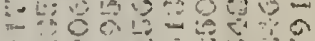

a 3

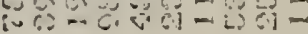

$i i i i i j i$

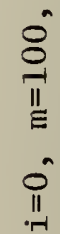

$\stackrel{0}{11}$

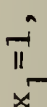

$\sqrt{-4}$

ริ)

疍

当

ปับ

站

త

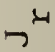

岁

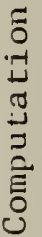

in

) 


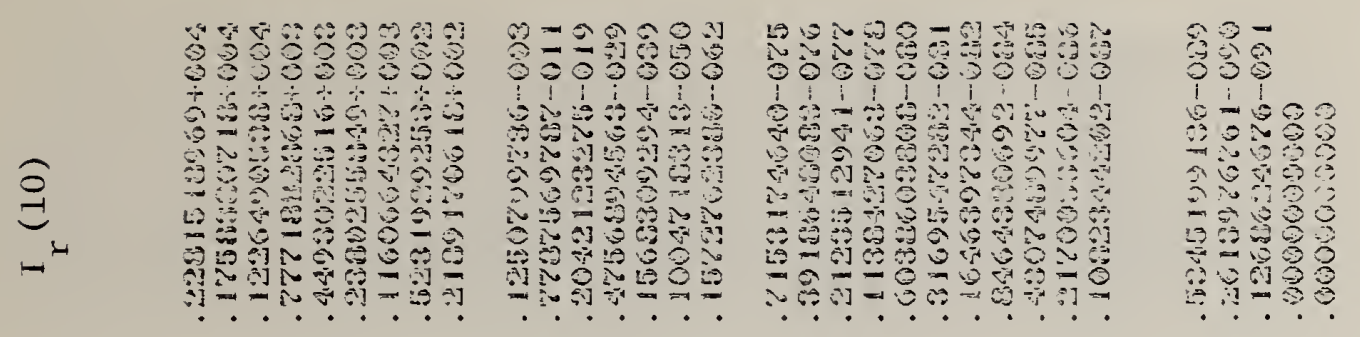

$\widehat{\underline{\theta}}$

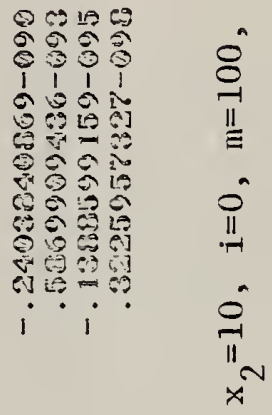

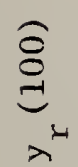

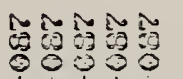

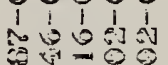

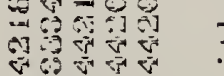

and 0

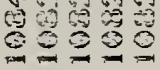

$\stackrel{\text { ก }}{I !}$

$0^{0}$



-

11119

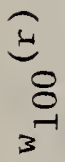

เด 5

की

चौ 40

और

दे is

$i$ i

$\stackrel{Ð}{\square}$

亏

$\stackrel{0}{-}$
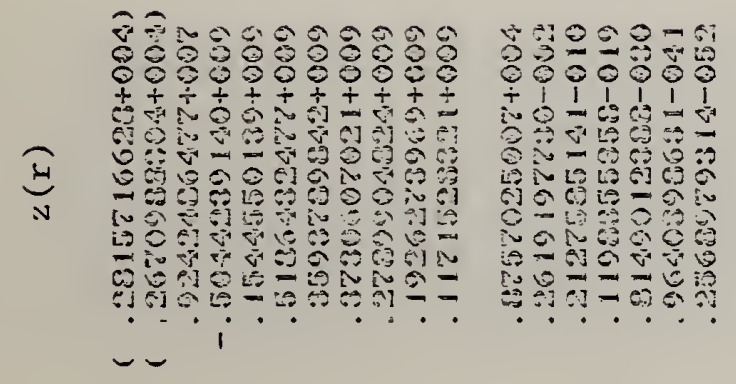

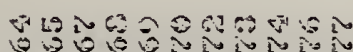

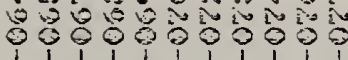

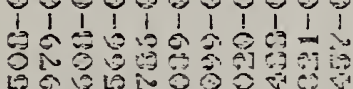

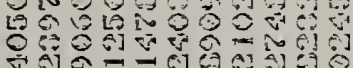

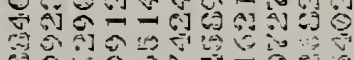

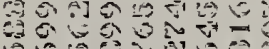
10504000

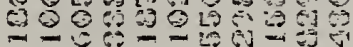

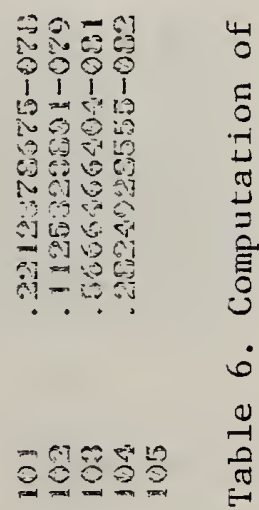

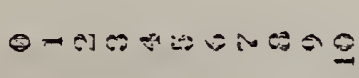

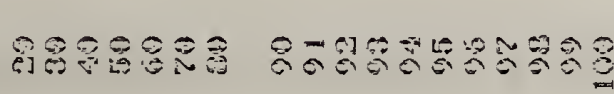



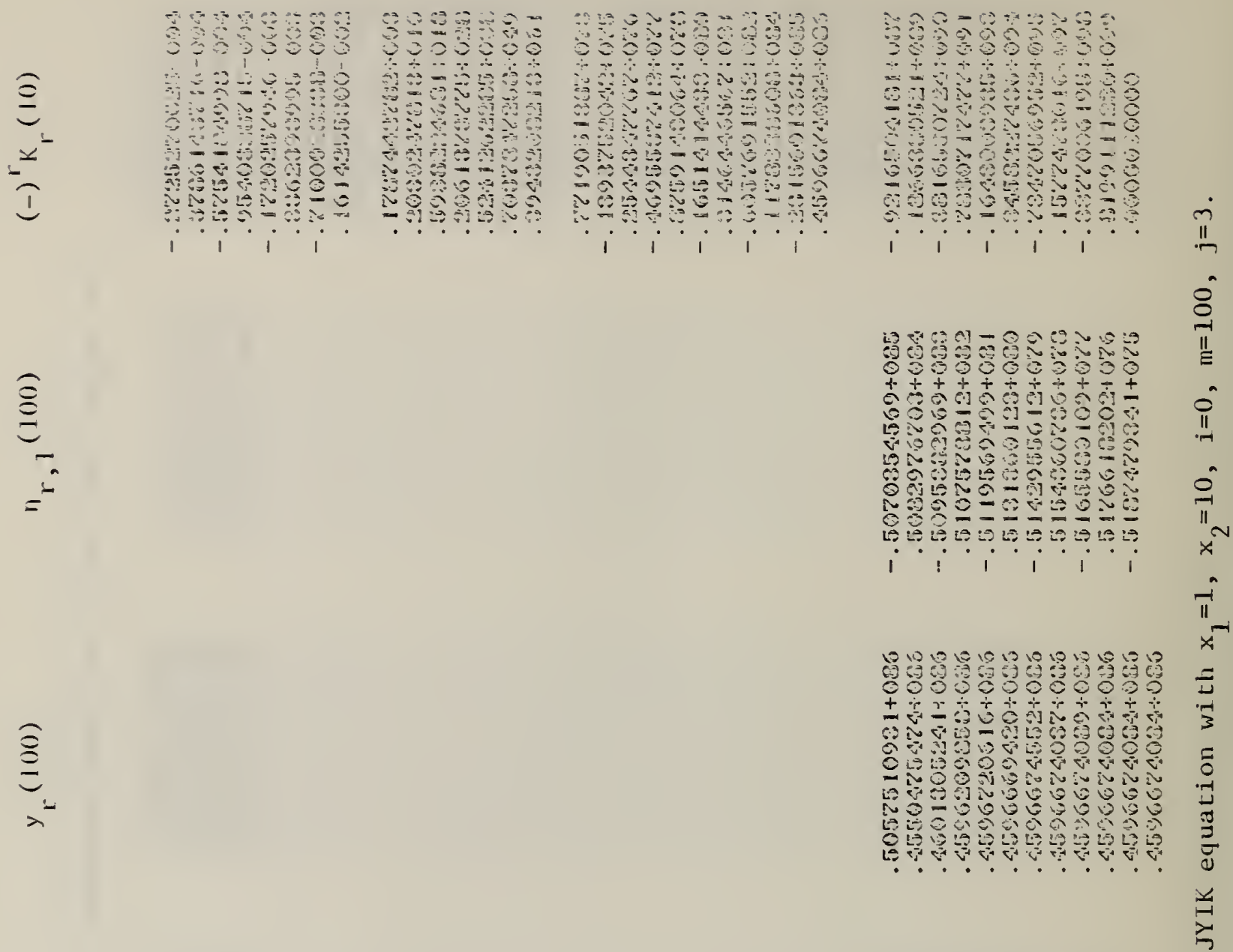

$\Xi$

ำ

$\stackrel{Ð}{\frac{1}{4}}$

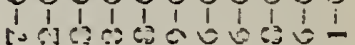

क然

20

Бо-

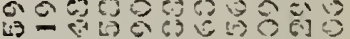

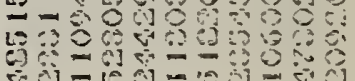

댄
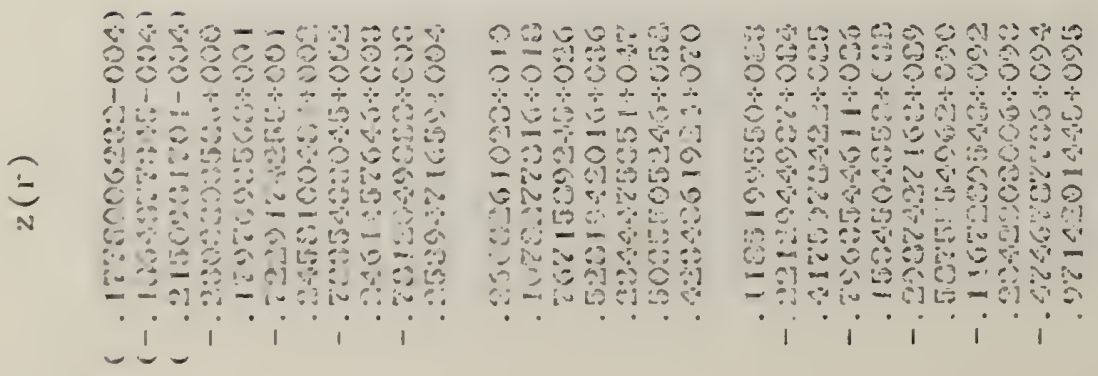

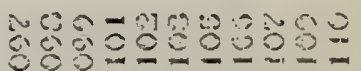

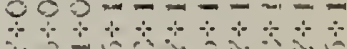

$40564 \div 4 \div$

c) 0 का

$\rightarrow$ 列

6.

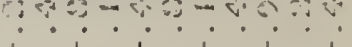

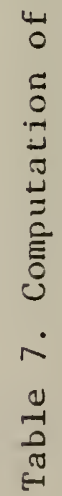

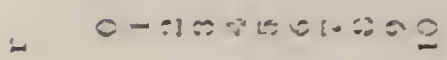

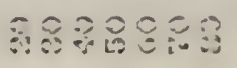

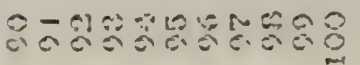




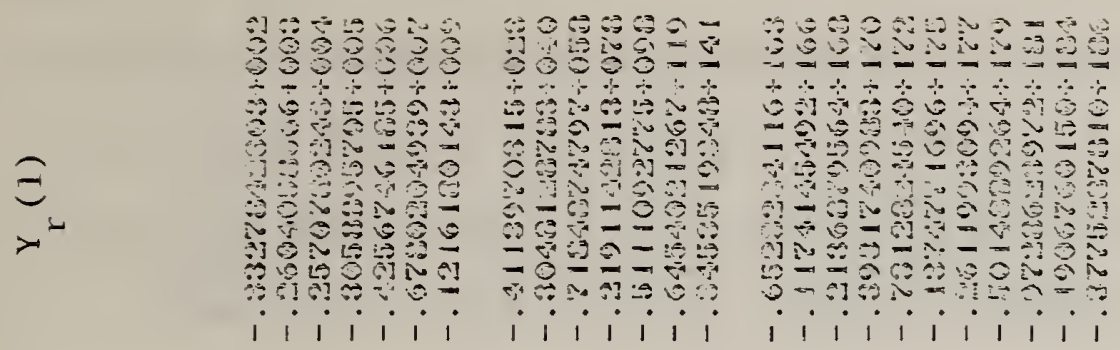

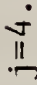

ํㅡㄹ

$\overbrace{11}^{\circ}$

$\frac{0}{11}$

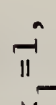

$x$

$\frac{\pi}{3}$

苟

$\stackrel{0}{\square}$

Е

$\Xi$
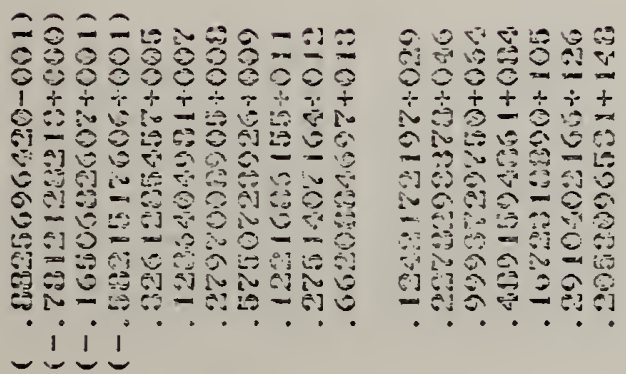

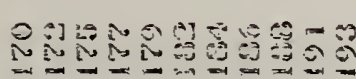

岁

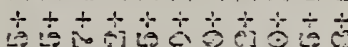

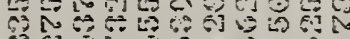

- $=6-2=0$

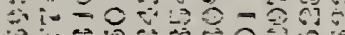

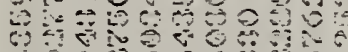

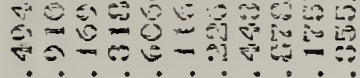

몰

$\infty$

$\stackrel{0}{\frac{1}{0}}$ 
Example 4.1.2 In this example we compute the solutions $I_{r}(1), J_{r}(10)$. $\mathrm{Y}_{\mathrm{r}}(10)$ and $(-)^{\mathrm{r}} \mathrm{K}_{\mathrm{r}}(1)$ of the homogeneous equation defined in Figure 6 , with $x_{1}=10, x_{2}=1$, for $r=0,1,2, \ldots, 100$. Both $I_{r}(1)$ and $\mathrm{K}_{\mathrm{r}}(1)$ are monotonic over this range of $\mathrm{r}$. The solutions $\mathrm{J}_{\mathrm{r}}(10)$ and $Y_{r}(10)$ are monotonic only for $10 \leq r \leq 100$; for lower values they oscillate with approximately the same amplitude. However, since there is no "crossing over" of growth behavior in the four solutions in the nonasymptotic region $0 \leq \mathrm{r} \leq 10$, we expect the algorithm to be stable with the following numbers of initial conditions: $j=1$ for $I_{r}(1)$, $j=2$ for $J_{r}(10), j=3$ for $Y_{r}(10)$ and $j=4$ for $(-){ }^{r_{r}}(1)$; compare (4.1.4). Tables 9 through 12 give the results of the computation of each of these solutions for selected values of $r$; see $\$ 4.0$ for a full description of these tables. Again, the stability of the algorithm for each solution is confirmed by comparison with Tables 9.4 and 9.11 of [16]. 

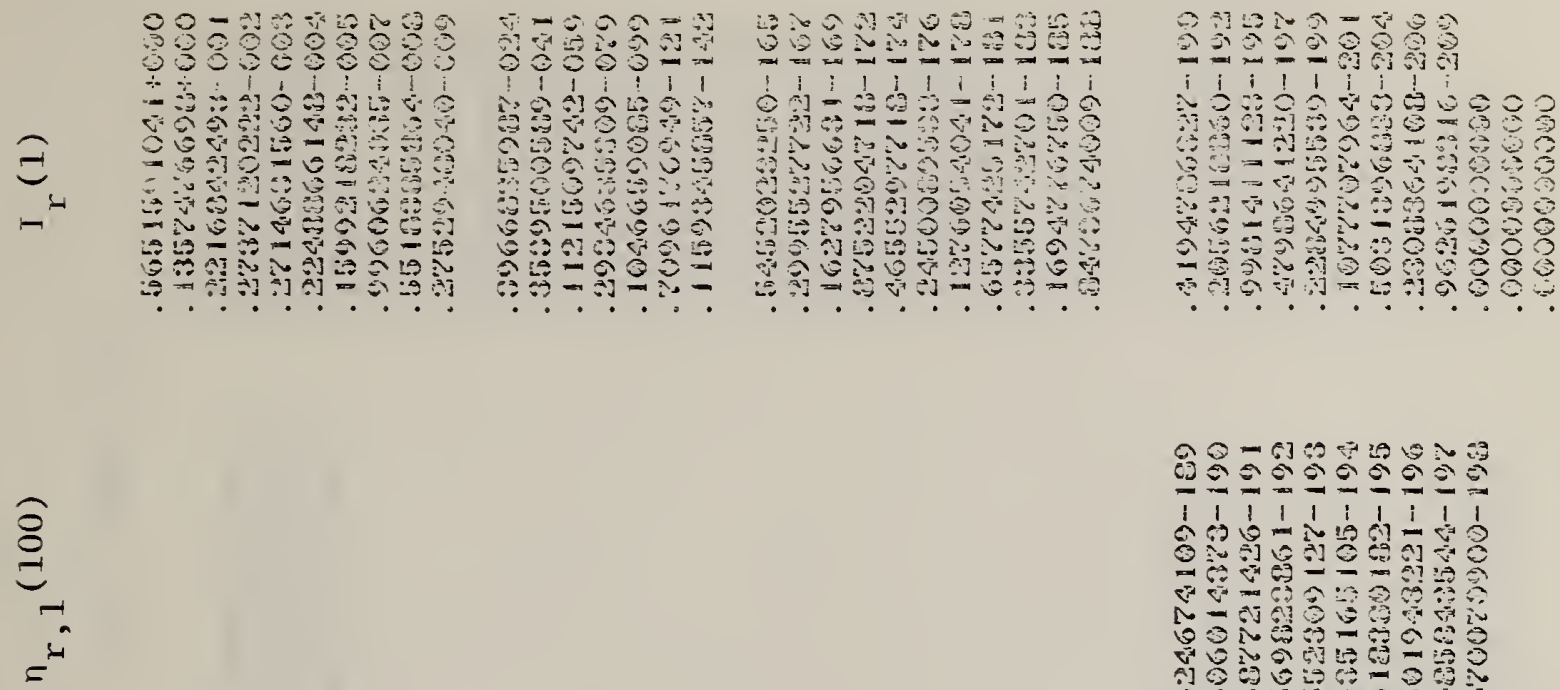

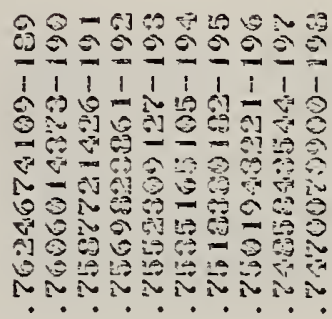

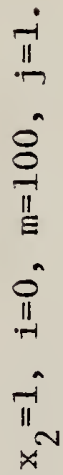

$\underbrace{\stackrel{0}{0}}_{\substack{0 \\ \lambda^{2}}}$

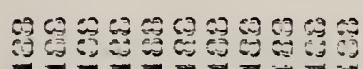

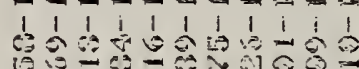

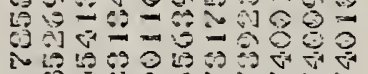

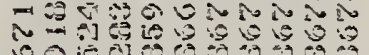

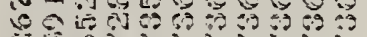
政

$\overbrace{3}^{\substack{0 \\ 3}}$

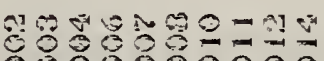
6.

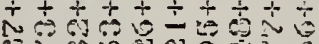

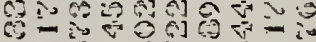

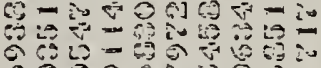
6920 00 80000

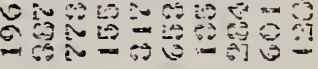

$0_{11}^{0}$ $-1$

苞

光

$\stackrel{g}{\square}$

క్

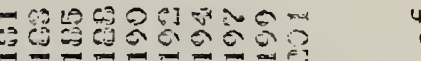

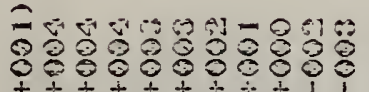

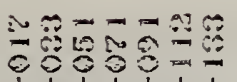

ㅂ. 1 1 11111

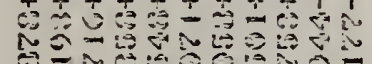

(7)

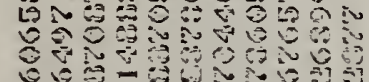

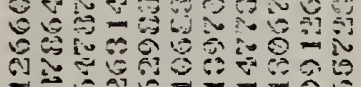

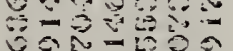

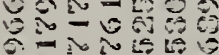

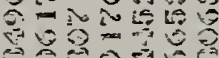
\%

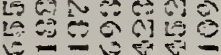
111717717

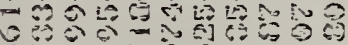
으의 cis

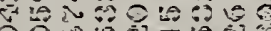

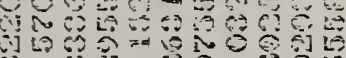

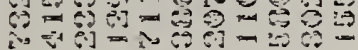
$-7-1-1 \div 1$

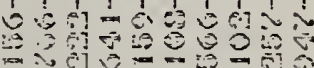
- 0 c

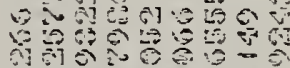

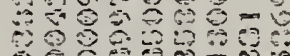
Ee con

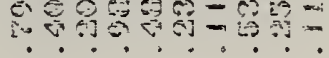

$0^{11}$

๘ 

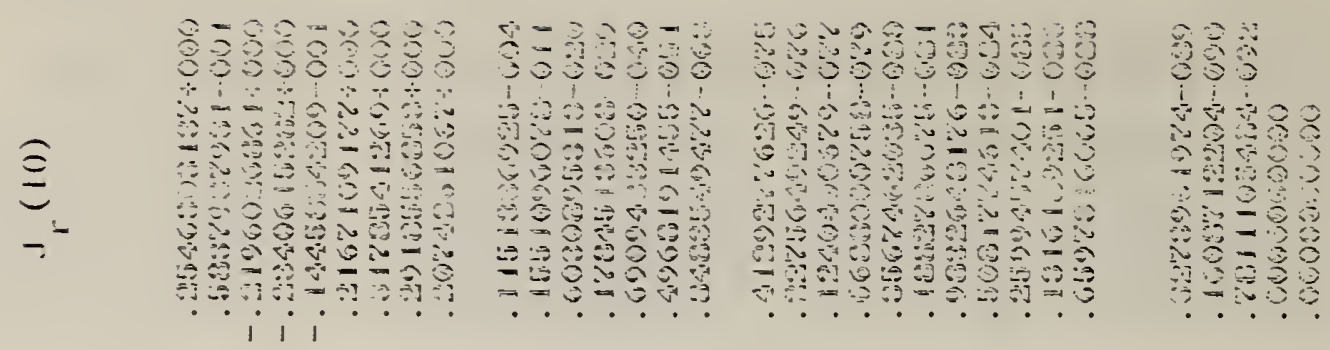

言-

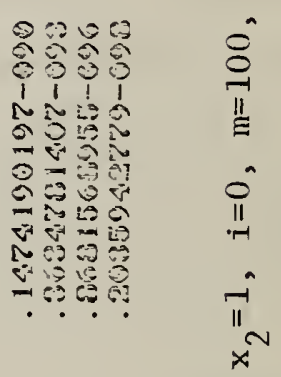

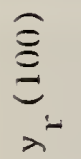

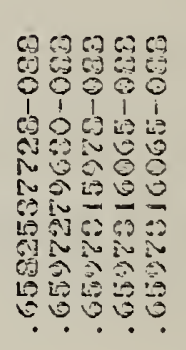

$0_{-1}^{0}$

5
-1
3
0
0
-1
0
0
0
0

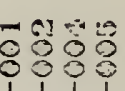

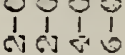

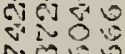

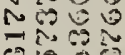

송

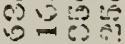

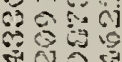

$\underset{3}{3}$

สำ.

ละ $+1++\div+4$

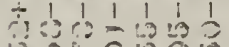

40.

c.

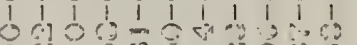

- $100=0$

I $\quad b=0$

300

$200-0=0$

o -10090

is 2

6

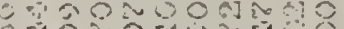

1: 0 a

o- 040

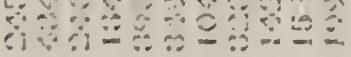

30

- $6=06006$

$\begin{array}{llll}1 & 1 & 1\end{array}$

जी

200

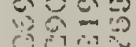

1 4

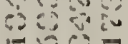

$02-$

$i_{i} i^{\prime} \dddot{i}_{i} i$

$i i i i i i$

1111

$\stackrel{i}{\|}$

in

省

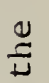

苾

$\stackrel{0}{5}$

4

동

$\stackrel{\circ}{\circ}$

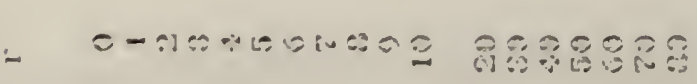

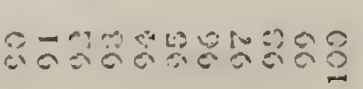

-

步 

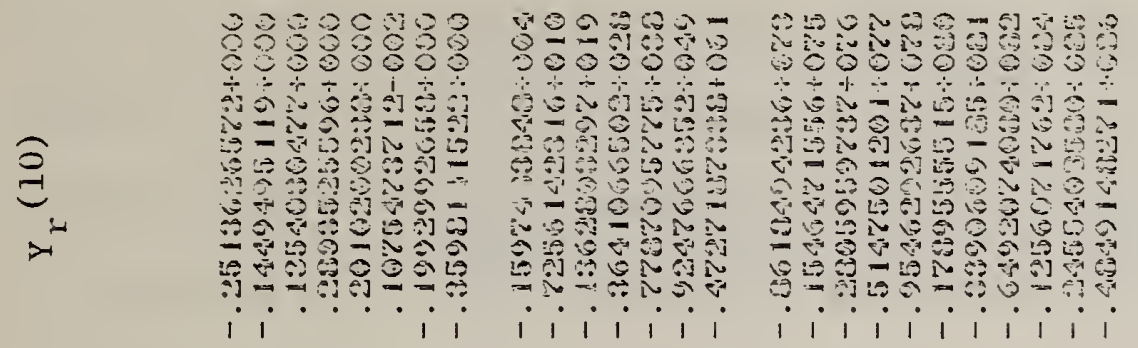

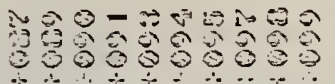

H

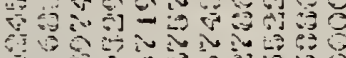

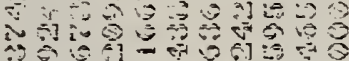

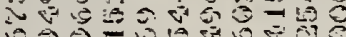

So

$i i i i i i i$

$\stackrel{\dot{n}}{\|}$

$\stackrel{8}{\circ}$
$=$

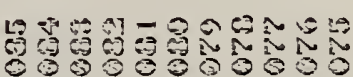

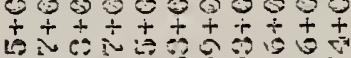

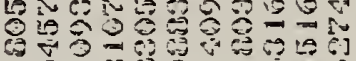

-

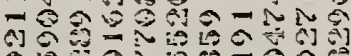

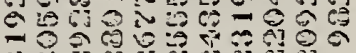

*을

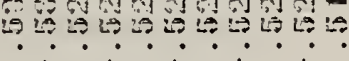

$\stackrel{8}{8}$

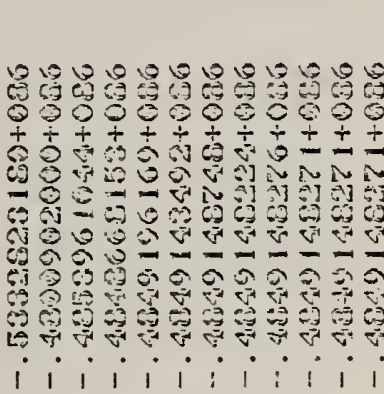

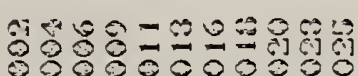

$000 \% 0000000$

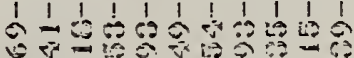

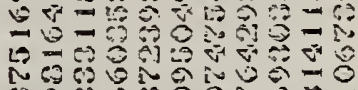

ติ

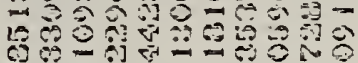

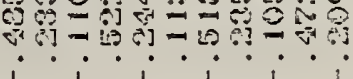

20ิ8

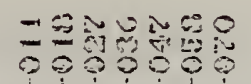

ใ.

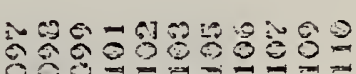
1 $11+4+4+4 \div$

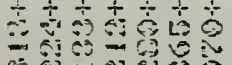

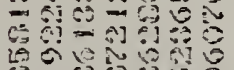

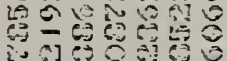

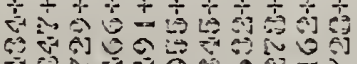

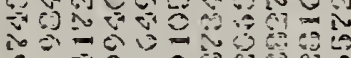

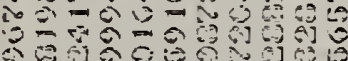
की 60

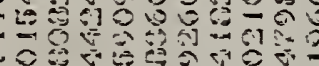
60

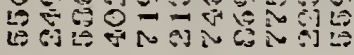
6? 0005 $-1,=-3=0$

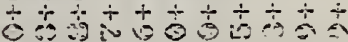
象前心

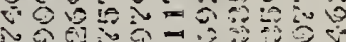

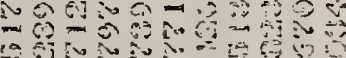

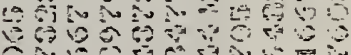
0050000 Q1 i $1 i i i i i i 1 i$

i i i i i i i 

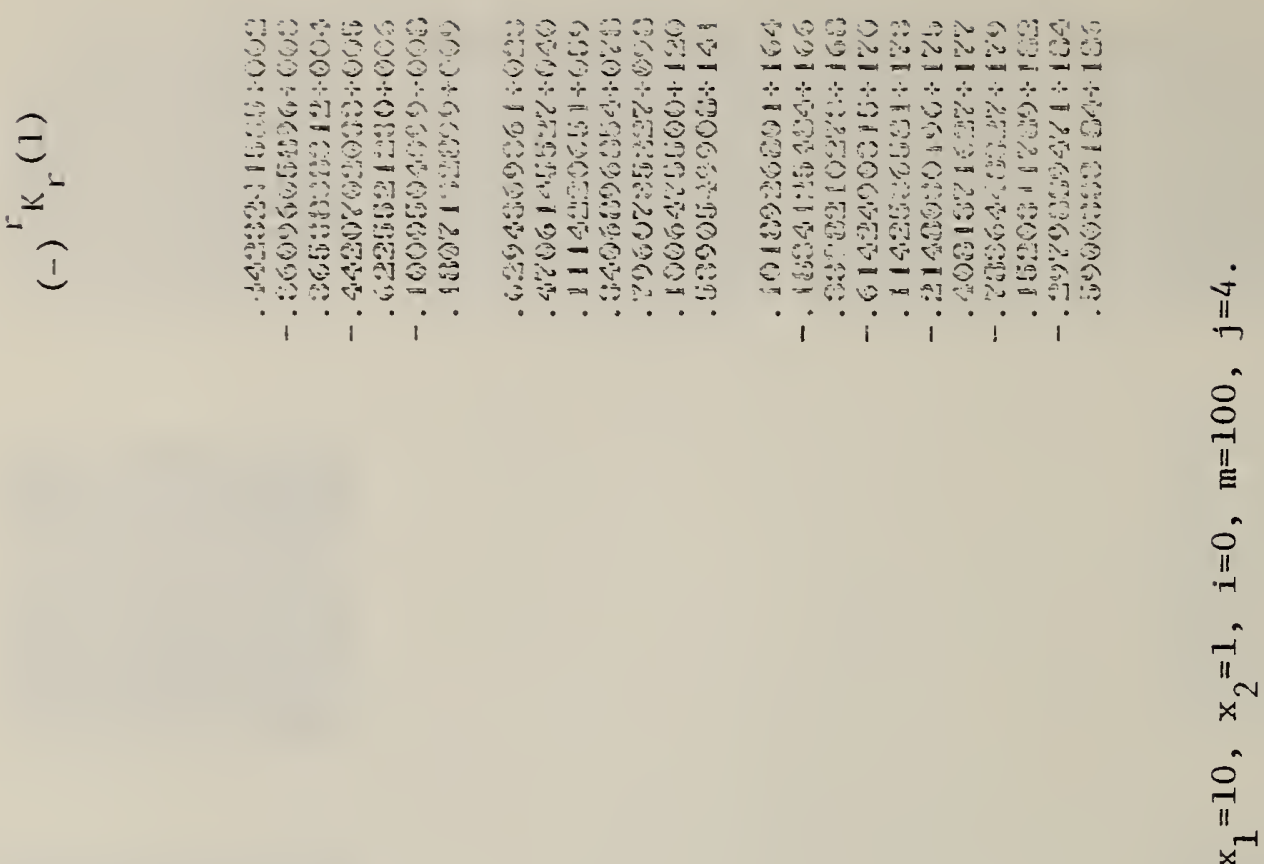

$\stackrel{5}{-1}$

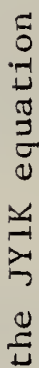

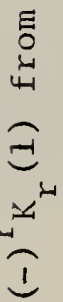

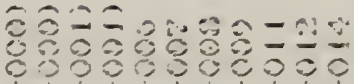
1

I 6

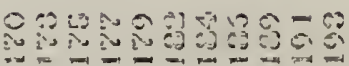
$-1-1,-1$ $+4+t+4+t$ 156 $60=0$ 的 c. c5:-3. -OC C C 1 e $209=020$

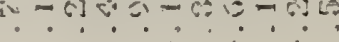

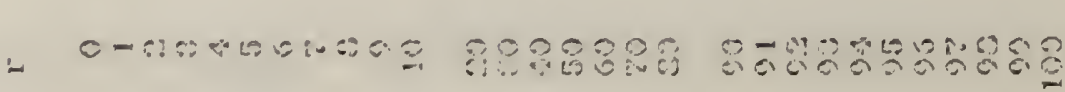
峲 
Example 4.1.3 In this example we compute the solutions ${ }^{\dagger} \mathrm{A}_{\mathrm{r}}(1), \mathrm{J}_{\mathrm{r}}(1)$, $I_{r}(1), Y_{r}(1)$ and $(-) K_{K}(1)$ of the homogeneous equation defined in Figure 6, with $x_{1}=1, x_{2}=1$, for $r=0,1,2, \ldots, 100$. Since each solution is monotonic (in magnitude) except possibly at the very beginning of the range of $r$, the algorithm is expected to be stable provided that the proper number of initial values is taken in each case. This number is $j=1$ for $A_{r}(1), j=2$ for $J_{r}(1)$ and $I_{r}(1)$, and $j=4$ for $Y_{r}(1)$ and $(-)^{r} K_{r}(1)$. However, in the case of $A_{r}(1)$, for reasons given earlier in this section, we take $j=2$ instead of $j=1$, thereby exchanging mild instability for a much improved rate of convergence.

Because of this mild instability, each individual rounding error, introduced at say $r=s$, is amplified in direct proportion to the number of steps taken beyond the point $s$, since in $(4.1 .7) \mathrm{A}_{\mathrm{r}} / \mathrm{I}_{\mathrm{r}}=0(1 / \mathrm{r})$. Thus, after 100 steps, approximately two decimal places of precision will be lost. On the other hand, if we were to take the number of initial conditions theoretically required (in this ase it is $j^{-=}=1$, since $A_{r}$ is dominated by all of the other homogeneous solutions), then the convergence would be extremely slow: A terminal point of the order of $10^{8}$ would be required to obtain 8 significant figures of accuracy in $A_{100}(1)$. Tables 13 through 17 give the results of these computations for selected values of $\mathrm{r}$; see $\$ 4.0$ for a full description of these tables. Again, these results agree with the entries in Table 9.4 and 9.11 of [16]. In the case of $A_{r}(1)$, the method we have used is equivalent to computing $J_{r}(1)$ and $I_{r}(1)$ as type 2 solutions and then forming their difference. The function $A_{r}(1)$ is defined by $A_{r}(1)=J_{r}(1)-I_{r}(1) ;$ compare
$(4.1 .6)$. 

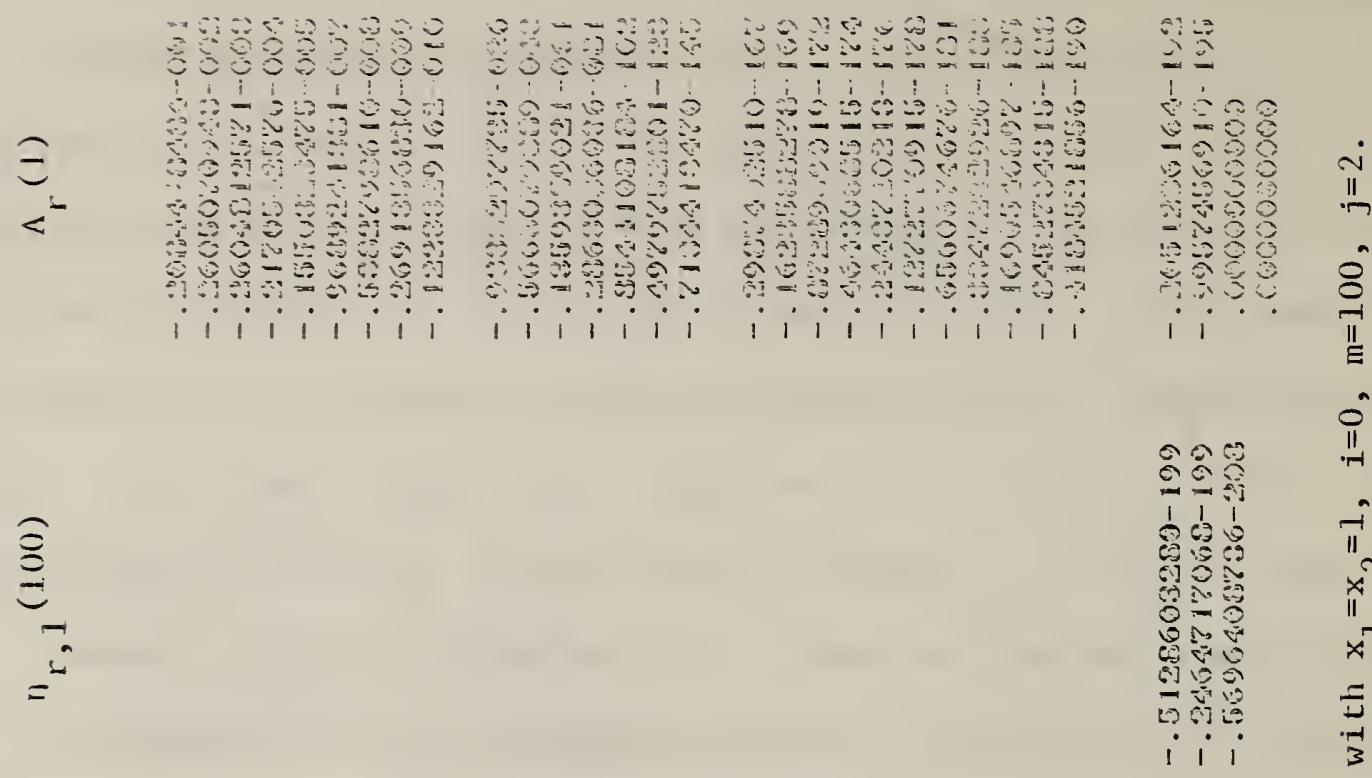

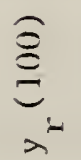

0000

1 i i i

正

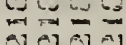

(1) 6 की 1

制

$\Leftrightarrow 3$

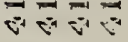

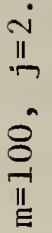

i i i

$\varliminf_{11}^{n}$

$\rightarrow$

"1)

$x^{-1}$

$\frac{\pi}{3}$

$\underset{3}{3}$
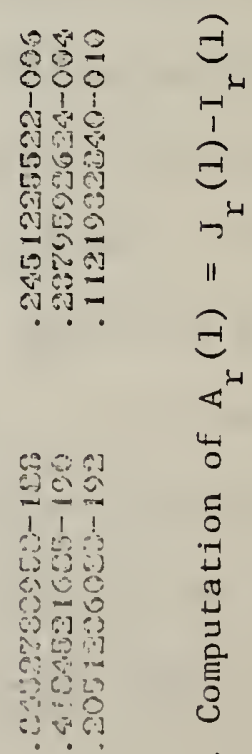

茨

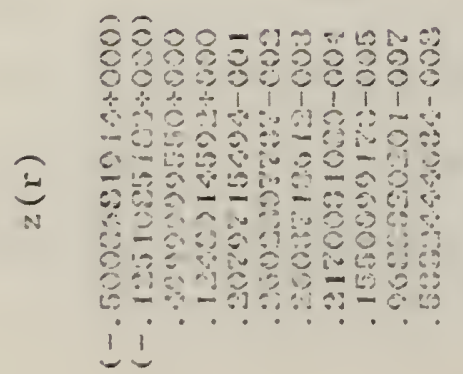

0000000

$\because$

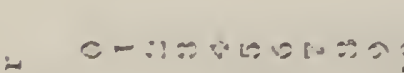
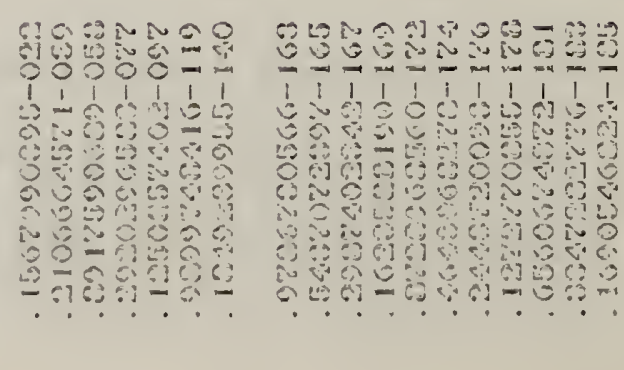

की 


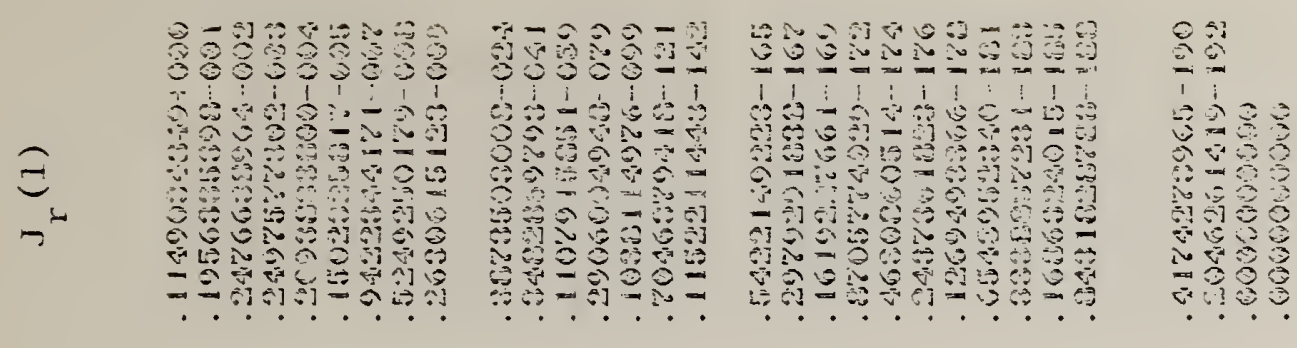

อ
=

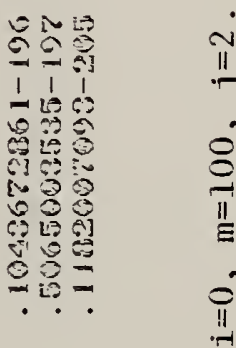

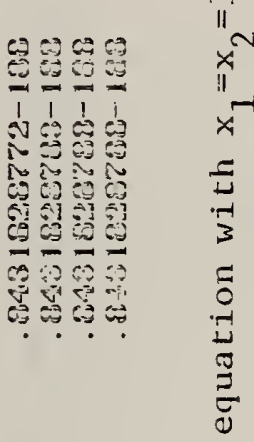

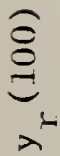

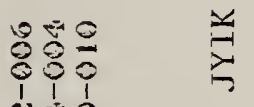

$\underbrace{\infty}_{3}$

बู่

t5

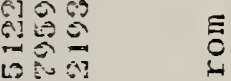

ดิ

త
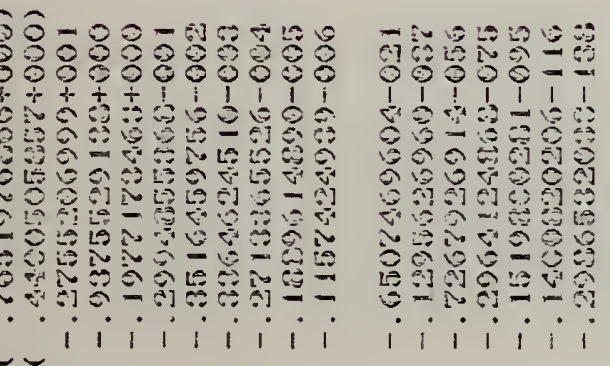

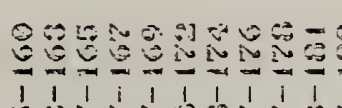

果影崖

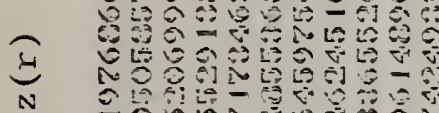

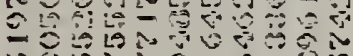

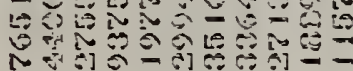

$\because \because i i i$ i i i

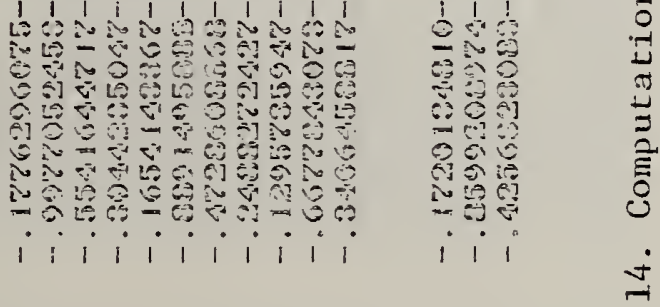

म 


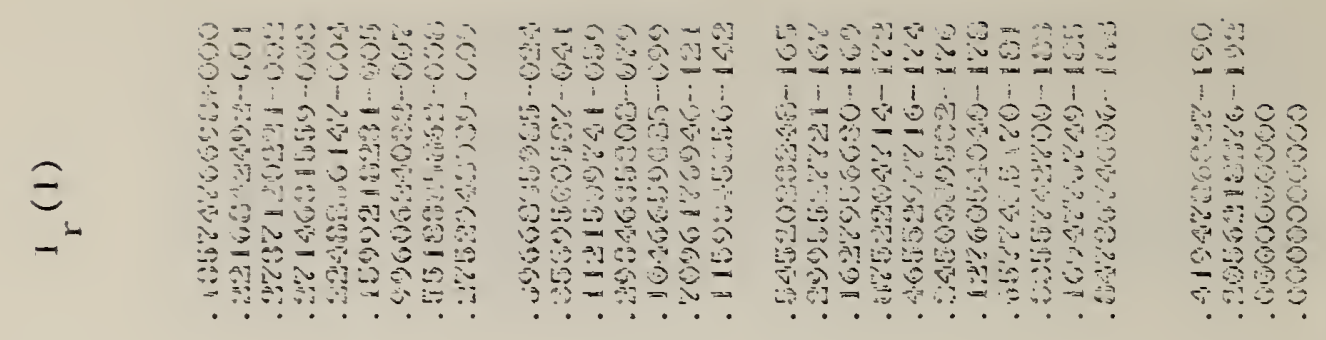

$\stackrel{\delta}{E}$
$=-$

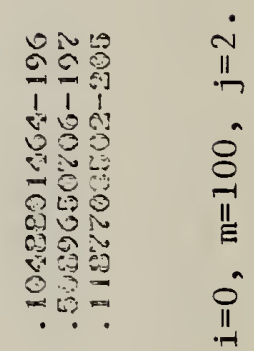

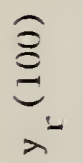

69.980

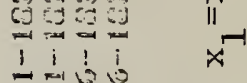

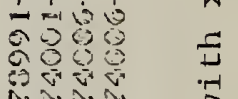

No i N

0 is 08

ब过

ป

3
3
3

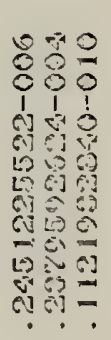

苛

$\stackrel{1}{1+}$

๕్

$\approx$

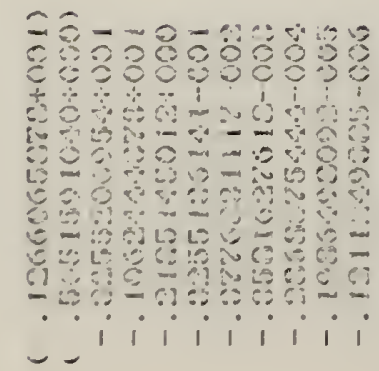

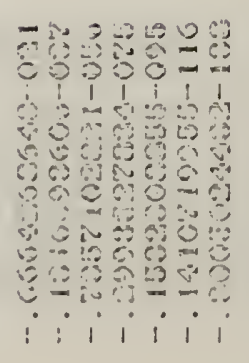

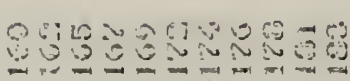

$-1-1 \quad-1-1-1$

6.

60 0030

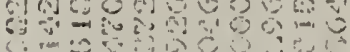

30

com $00620=$

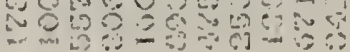

ii i i i i i

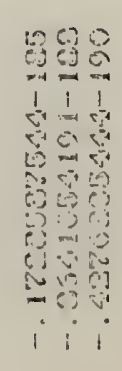

岁

5

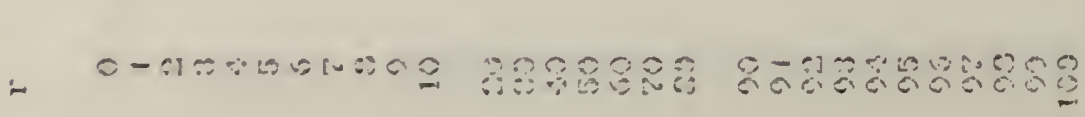

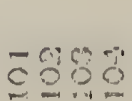

苟 

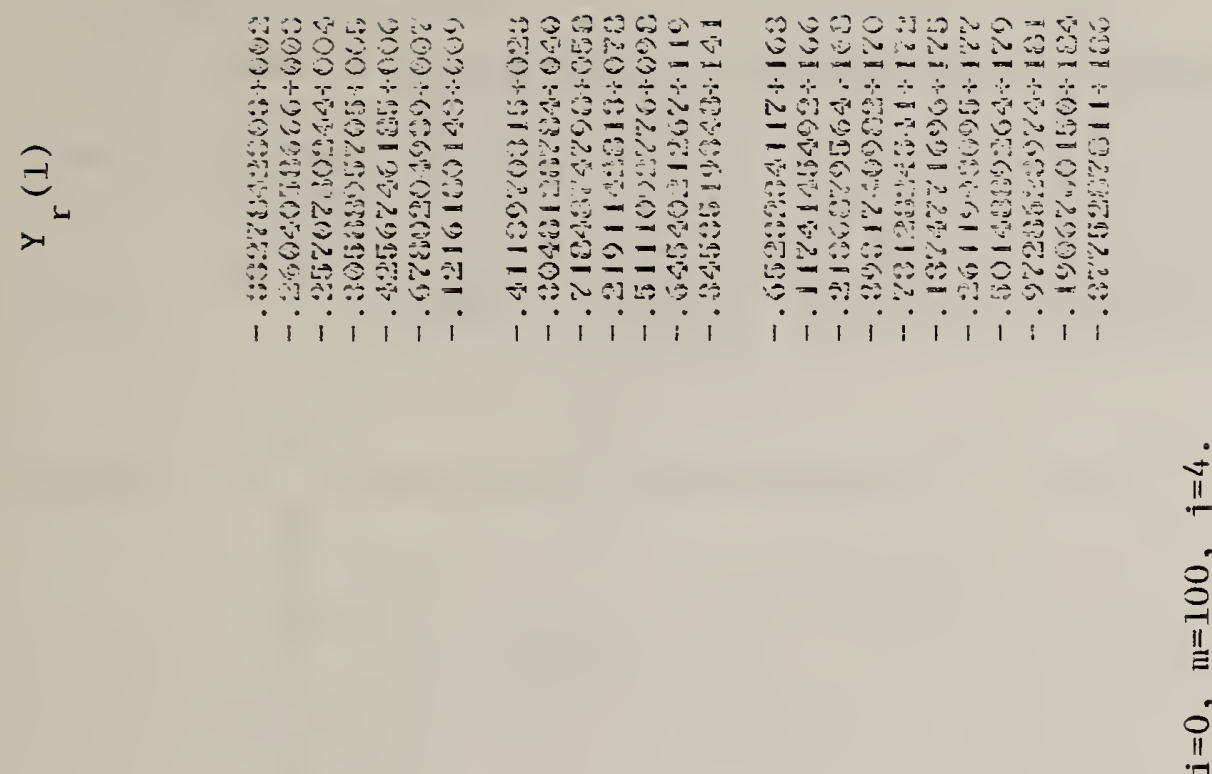

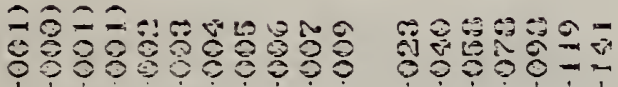

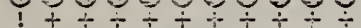

$4+5+1$

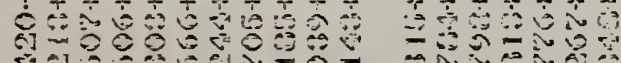

ב્ఝ

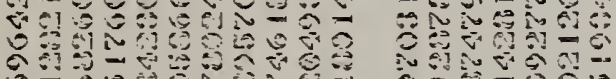

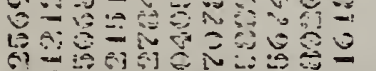

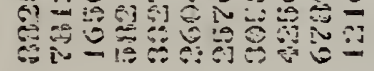

i 1 i 1 i i i i i

-

$=030=$

F⿻一𠃋十

1

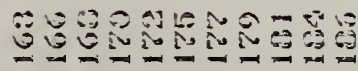

$+4+4+4+4+4$

- 060

$\overline{6} \mathrm{O}$

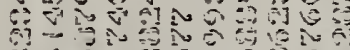

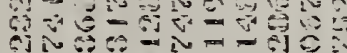

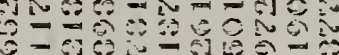

$\stackrel{\varrho}{\varpi}$

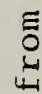

$\underset{7}{-1}$

茨

تే

4

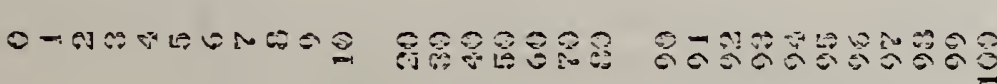

$\underset{\frac{0}{\pi}}{\stackrel{0}{=}}$ 

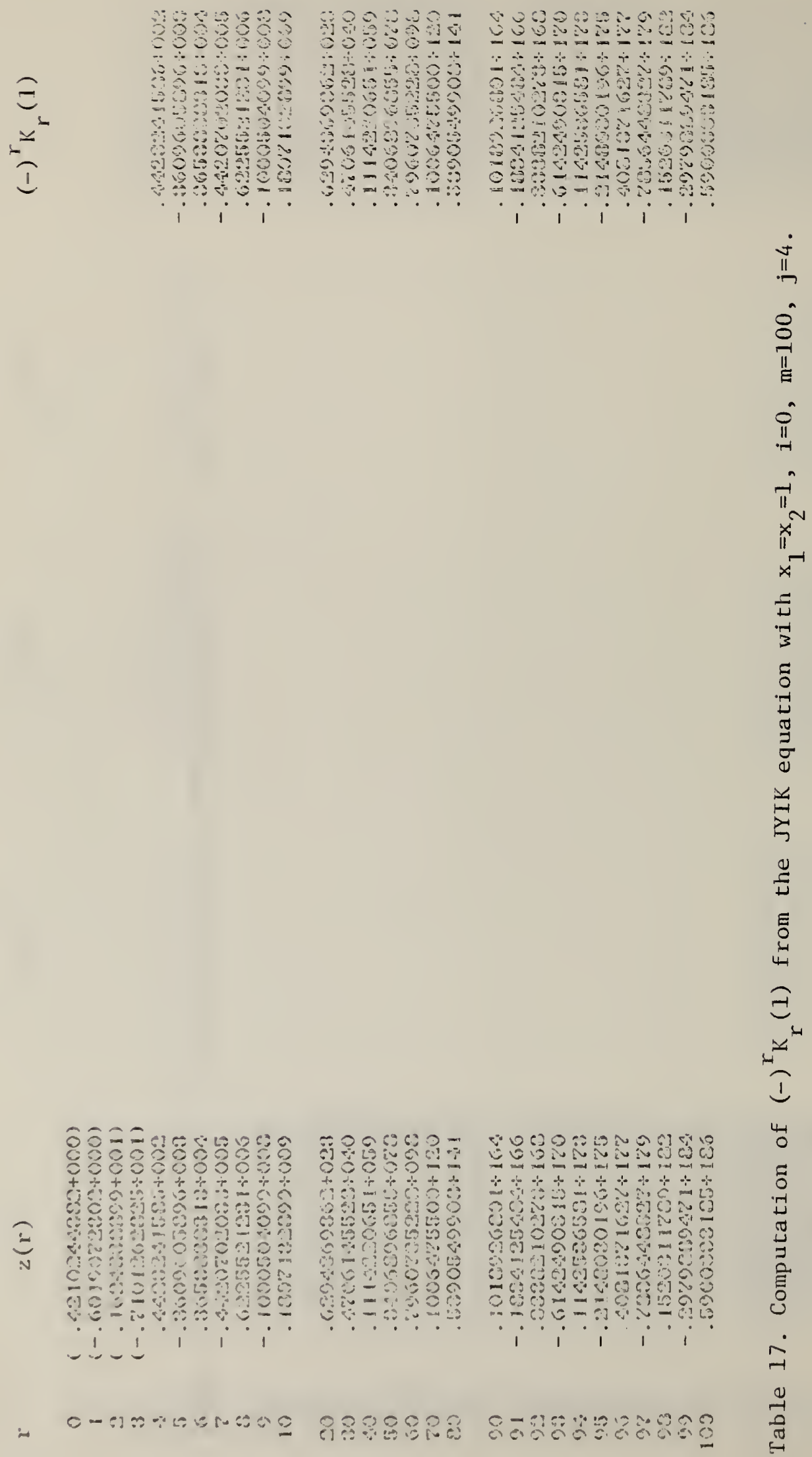


\subsection{Examples Involving the Inhomogeneous JYIK Equation}

For fixed $x$ and large $r$ the Anger-Weber function has the asymptotic forms

$$
E_{2 r}(x) \sim \frac{2 x}{\left(4 r^{2}-1\right) \pi} \quad, E_{2 r+1}(x) \sim \frac{2}{(2 r+1) \pi} ;
$$

compare [21]. Comparison of $(4.2 .1)$ with $(4.1 .1)$ and $(4.1 .2)$ shows that (4.2.2) $\left\{\begin{array}{l}I_{r}\left(x_{2}\right) \\ J_{r}\left(x_{1}\right)\end{array}\right\}<E_{r}(x)<\left\{\begin{array}{l}(-)^{K_{K}}\left(x_{2}\right) \\ Y_{r}\left(x_{1}\right)\end{array}\right\}, \quad r \rightarrow \infty$,

for any given set of values of $x_{1}, x_{2}, x$. Thus $E_{r}(x)$ is a solution of type 2 of the first inhomogeneous equation defined in Figure 6 and the correct number of initial values of $E_{r}(x)$ to be used in every case is $j=2$.

The asymptotic form of the Struve function, also taken from [21], is

$$
H_{r}(x) \sim \frac{x}{\sqrt{2} \pi r}\left(\frac{e x}{2 r}\right)^{r}
$$

for fixed $\mathrm{x}$ and large $\mathrm{r}$. Comparison of (4.2.3) with (4.1.1) and (4.1.2) shows that

$$
H_{r}(x) \sim \frac{x}{\sqrt{\pi r}}\left\{\begin{array}{c}
I_{r}(x) \\
J_{r}(x)
\end{array}\right\}, r \rightarrow \infty .
$$

Thus $H_{r}(x)$ is a solution of type 1 of the second inhomogeneous equation defined in Figure 6 when $x_{1}=x_{2} ;$ compare (4.1.8). However, since the separation of $\mathrm{H}_{\mathrm{r}}(\mathrm{x})$ from solutions of type 2 is even weaker than 
that of the solution $A_{r}(x)$ defined by (4.1.6) it is again advantageous in most applications to specify the number of initial values appropriate to solutions of type 2 , i.e., $j=2$ instead of $j=1$; compare Example 4.1.3.

Example 4.2.1 For the inhomogeneous fourth-order difference equations defined in Figure 6, we compute $E_{r}(1)$ with $x_{1}=x_{2}=1$ for $r=0,1,2$, $\ldots, 100$; and $\mathrm{H}_{\mathrm{r}}(0.1)$ with $\mathrm{x}_{1}=\mathrm{x}_{2}=0.1$, for $\mathrm{r}=0,1,2, \ldots, 50$. The appropriate number of initial values in these cases is $j=2$ for $E_{r}(1)$ and $j=1$ for $H_{r}(0.1)$. However, for reasons given above we actualiy use $j=2$ for $H_{r}(0.1)$. Initial values for $E_{r}(1)$ and $H_{r}(0.1)$ were computed from series expansions given in [21] and checked against values Eound in [21] and [23]. Tables 18 and 19 give the results of these computations for selected values of $r$; see $\$ 4.0$ for a full description of these tables. They agree with the 10-figure values of $E_{r}(1)$ and $H_{r}(0.1)$ $r=2,3,4,5$, given in [23]; the 7 through 9 -figure values of $E_{r}(1)$, $r=2,3, \ldots, 10$, given in $[21]$; and the 9 -figure values of $H_{r}(0.1)$, $r=1,2, \ldots, 13$, given in [21]. In addition, they agree with series calculation of the functions to 10 significant figures in the case of $E_{r}(I)$, $r=2,3, \ldots, 100$, and to 9 significant figures in the case of $\mathrm{H}_{\mathrm{r}}(0.1)$, $r=2,3, \ldots, 50$. The loss of precision in the struve function computation is due to mild instability which is acceptable in lieu of impossibly slow convergence.

We restrict the sequence of $H_{r}(0.1)$ to $r \leq 50$ in order to avoid underflow in the computer. As suggested at the end of $\$ 4.0$, this difficulty could be overcome by using the software described in [11]. 


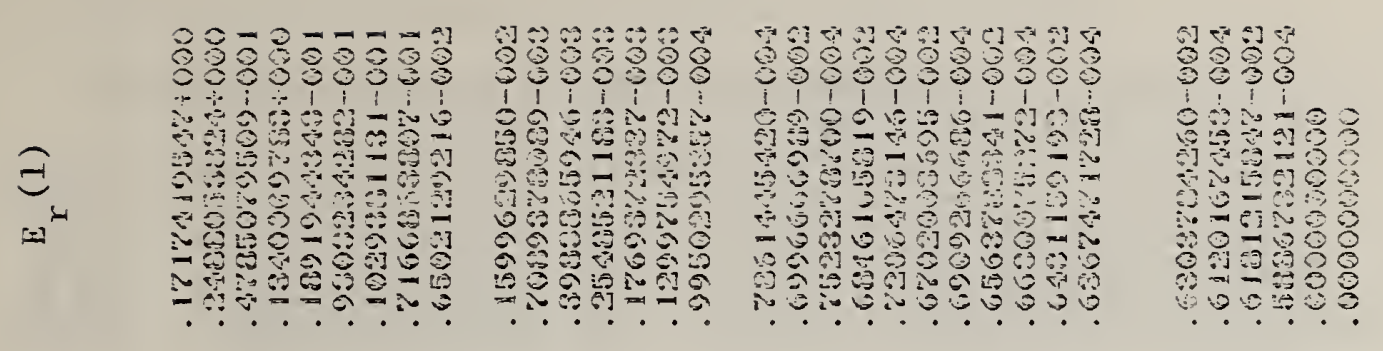

$\stackrel{8}{8}$
$=-1$
$=-1$
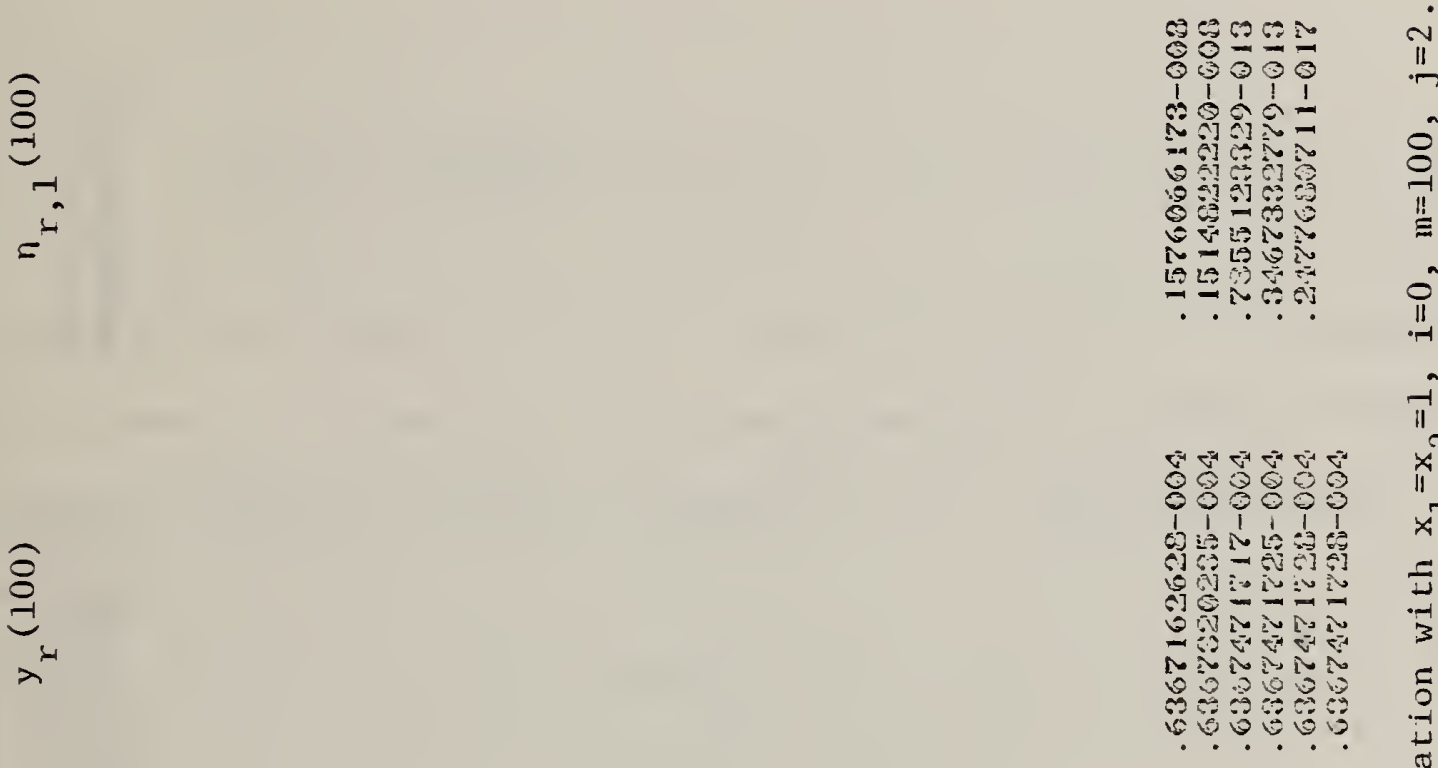

201

$\underset{3}{3}$

웅요응

1 $\begin{array}{lll}1 & 1 & 1 \\ 4 & 0 & 1\end{array}$

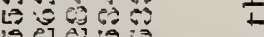

29.

Ai

ten

งิ

ถิ

Ð

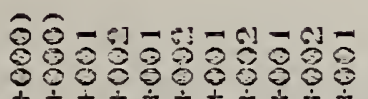

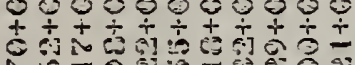

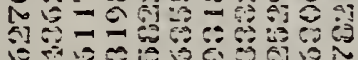

$\underset{N}{\overparen{E}}$

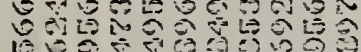
to 0 .

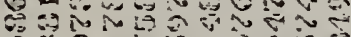

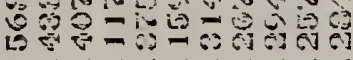
i-iiiiiii
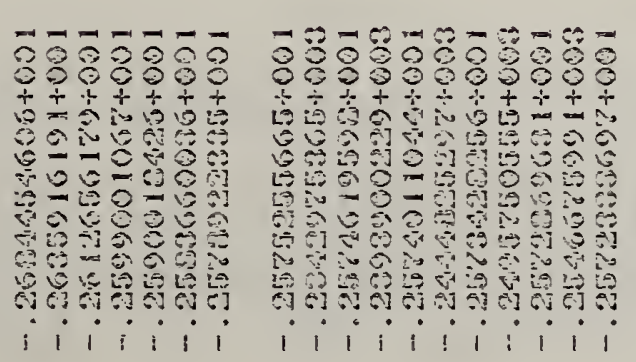

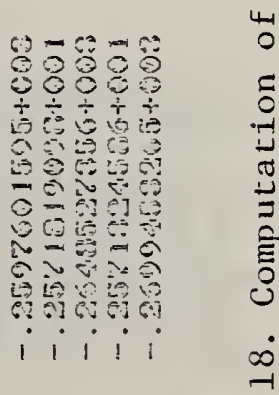

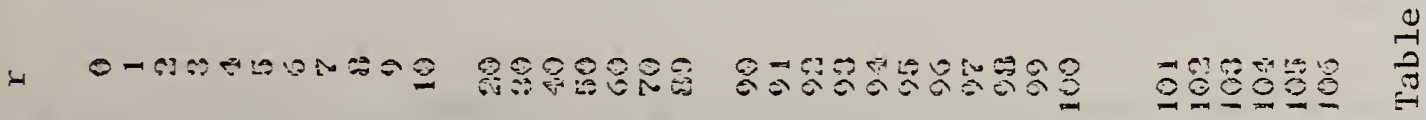



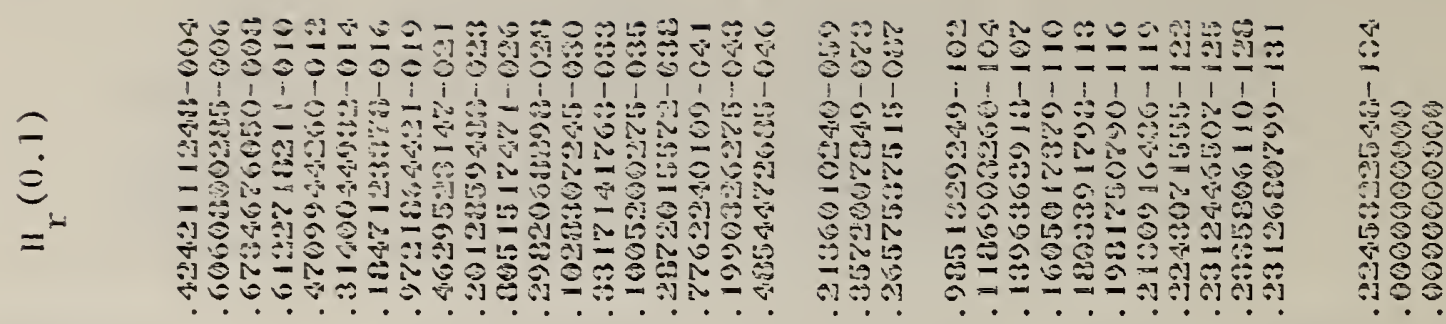

$\underset{ \pm}{E}$
$=-$

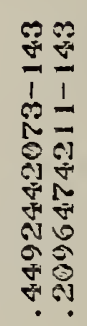

II

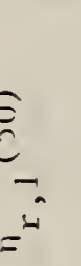

$\underset{7}{\sqrt{0}}$

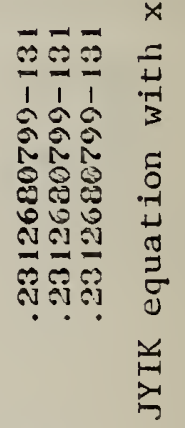

$\underbrace{30}_{3}$

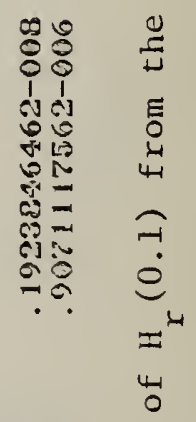

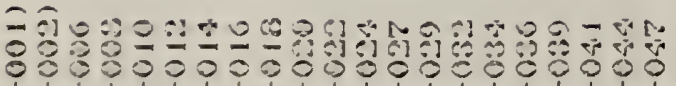

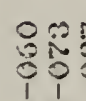

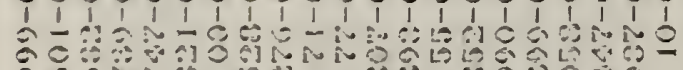

I

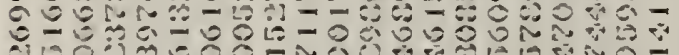
正势

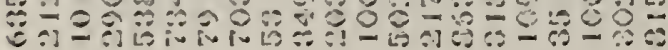
$\smile \smile$

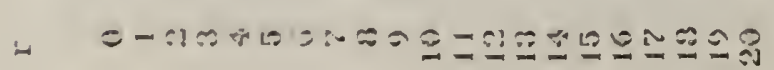
1 6द⿱ io 0 8 is: is (i)

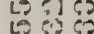
Lิ i 1 i

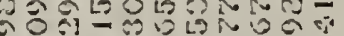
- 0 iv

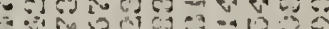

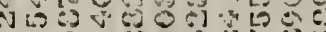
icos $5: 300=61610101$ $\begin{array}{llllllllll}1 & 1 & 1 & 1 & 1 & 1 & 1 & 1 & 1 & 1\end{array}$

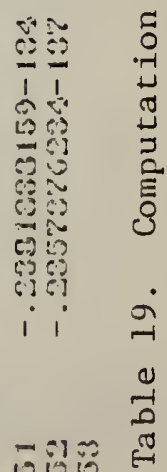

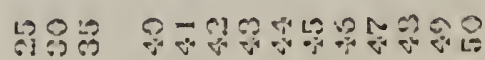
เร 12 L2 


\subsection{Examples Involving the Homogeneous JYPQ Equation}

For large values of $r$ and fixed values of $u$ and $x$, the associated Legendre functions have the asymptotic forms

$$
\text { (4.3.1) } \quad \mathrm{P}_{\mathrm{r}}^{\mu}(\mathrm{x})=\frac{\Gamma(r+\mu+1)}{\Gamma(r+3 / 2)}\left(\frac{1}{2} \pi \sin \theta\right)^{-1 / 2} \cos \left(r \theta+\frac{1}{2} \theta-\frac{1}{4} \pi+\frac{1}{2} \mu \pi\right)+O\left(\frac{1}{r}\right)
$$

and

(4.3.2) $\quad Q_{r}^{\mu}(x)=\frac{\Gamma(r+\mu+1)}{\Gamma(r+3 / 2)}\left(\frac{\pi}{2 \sin \theta}\right)^{1 / 2} \cos \left(r \theta+\frac{1}{2} \theta+\frac{1}{4}+\frac{1}{2} \mu \pi\right)+O\left(\frac{1}{r}\right)$

where $x=\cos \theta$ with $0<\theta<\pi ;$ see [16, Chapter 8]. Consequently, for values of $x$ less than 1 in magnitude, neither associated Legendre function dominates the other. Comparing (4.3.1) and (4.3.2) with (4.1.1) we obtain

$$
J_{r}\left(x_{1}\right)<\left\{\begin{array}{c}
P_{r}^{\mu}\left(x_{2}\right) \\
Q_{r}^{\mu}\left(x_{2}\right)
\end{array}\right\}<Y_{r}\left(x_{1}\right), \quad r \rightarrow \infty
$$

for all admissible values of $\mu, x_{1}, x_{2}$. Thus the JYPQ equation has three distinct types of solution, and the dimensions of the subdominant subspaces of types 1,2 and 3 are 1,3 and 4 respectively; this contrasts with $(4.1 .3),(4.1 .4)$, and (4.1.8).

Example 4.3.1 In this example we compute the solutions $\mathrm{J}_{\mathrm{r}}(1), \mathrm{P}_{\mathrm{r}}(0.5)$, $Q_{r}(0.5)$ and $Y_{r}(1)$ of the homogeneous equation ${ }^{\dagger}$ defined in Figure 7 , with $u=0, x_{1}=1, x_{2}=0.5$, for $r=0,1,2, \ldots, 100$. Since these solutions exhibit their characteristic asymptotic separation, described by (4.3.3), from the very beginning of this range of $r$, the algorithm

\footnotetext{
Tn accordance with custom, when the order of the associated Legendre functions is zero we drop the superscript in the notation.
} 
will be stable provided the proper number of initial values is used in each case. This number is $j=1$ for $J_{r}(1), j=3$ for $P_{r}(0.5)$ and $Q_{r}(0.5)$, and $j=4$ for $Y_{r}(1)$.

Table 20 gives the numerical coefficients of the JYPQ operator with $\mu=0, x_{1}=1$ and $x_{2}=0.5$ for $r=0,1,2, \ldots, 104$. Tables 21 and 22 give the numerical coefficients of the $(0,1)$-factorization and the $(0,3)$ iactorization. Since the $(i, j)$-factorization $D_{j}^{i}=A_{j}^{i} B^{i+j}$ produced by the test program has the principal diagonal of $A_{j}^{i}$ equal to $(1,1,1, \ldots)$, we do not include values of $a_{j}(r)$.

Tables 23 through 26 give the results of the computation of the four desired solutions for selected values of $\mathrm{r}$; see $\$ 4.0$ for a full description of these tables. Initial values were obtained from Table 9.4 of [16] in the case of the Bessel functions and by hand calculation of the explicit elementary forms given in [16], Section 8.4 in the case of the Legendre Functions. The computed Bessel function values agree satisfactorily with the values given in [16]. The Legendre function values were compared with daca available for $r \leq 10$ in [16] and (in the case of the $\mathrm{P}_{\mathrm{r}}(0.5)$ ) [17]. Agreement is satisfactory to the precision available in these references; this ranges from 6 to 8 significant figures. In addition, all of the compuced Legendre function values agree satisfactorily with double-precision values computed by an unpublished program of Dr. J. M. Smith of the National Bureau of Standards. This is sufficient to demonstrate stability. 


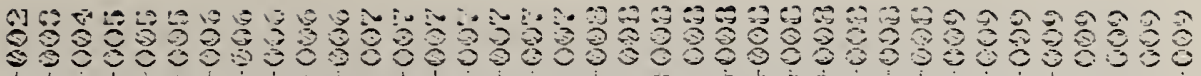

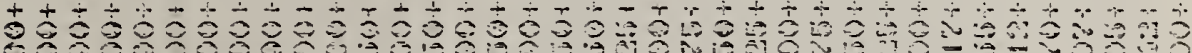

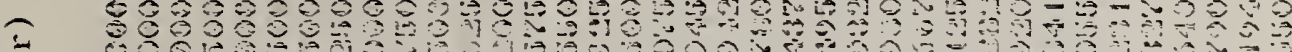
88050

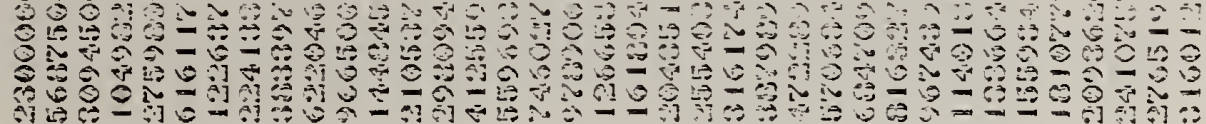

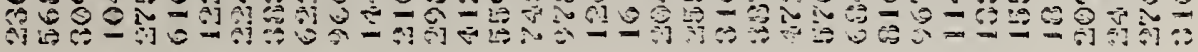
i i i i i i i i i i i i i i i i i i i i i i

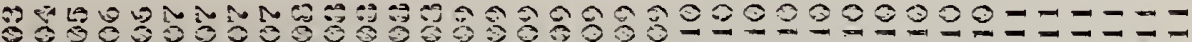
\%

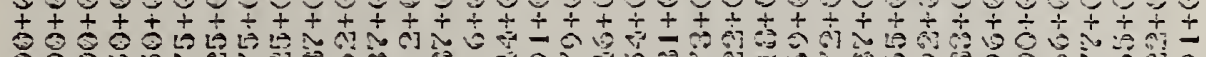
守8 I 800 m ha

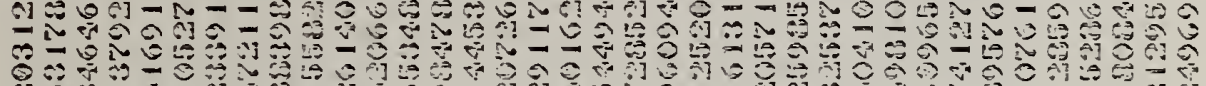

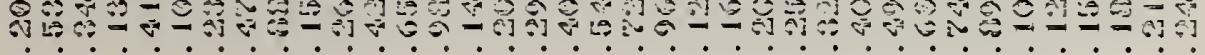

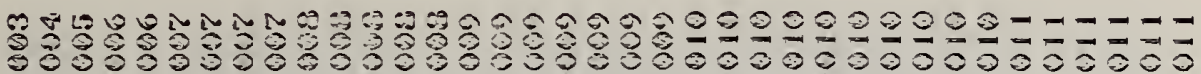

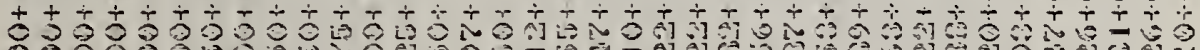

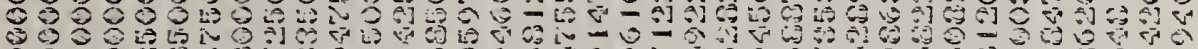

I O

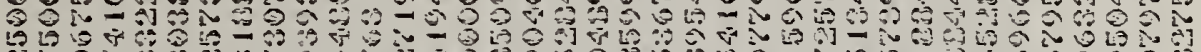

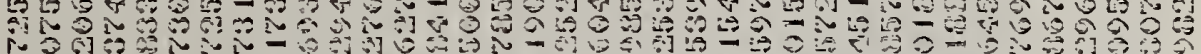

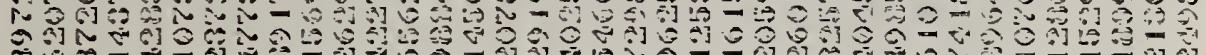

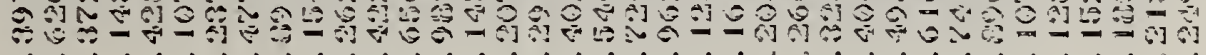

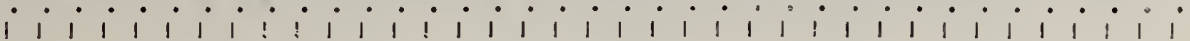

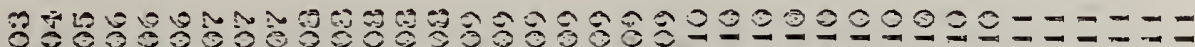

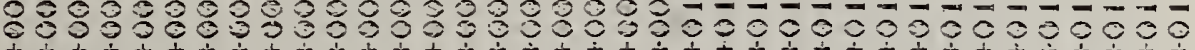

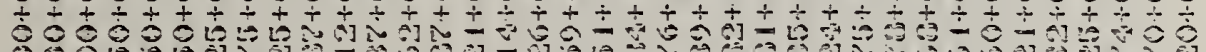

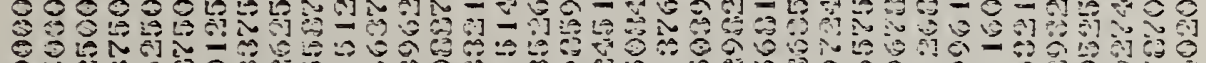

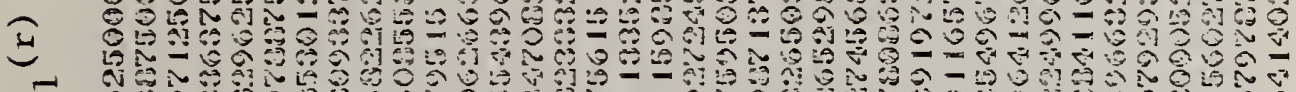
ôn

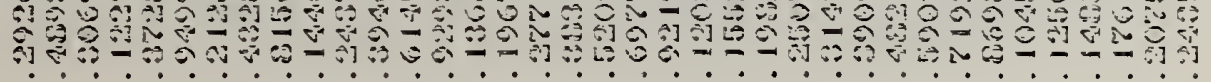

47

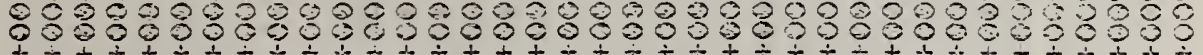

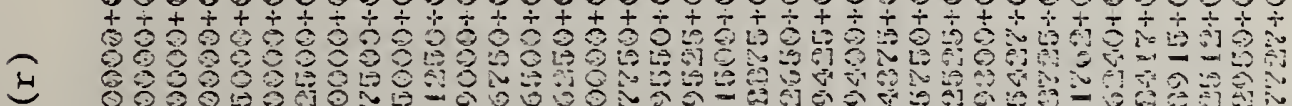

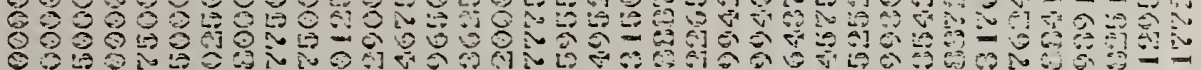
Sos

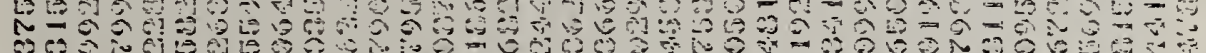

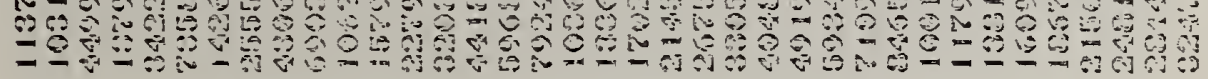
i i i i i i i i i ili i i i i i i i i i i i i

$\dot{8}$

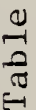




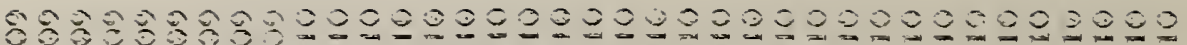

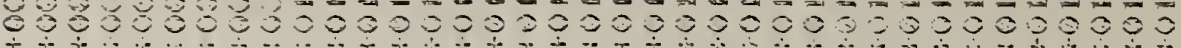

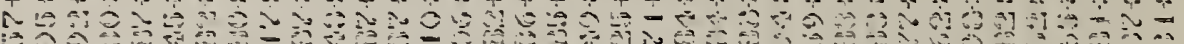
I

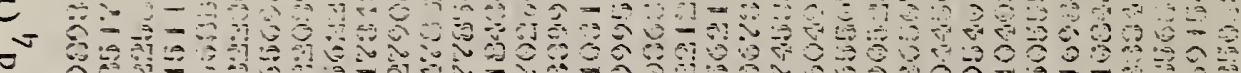

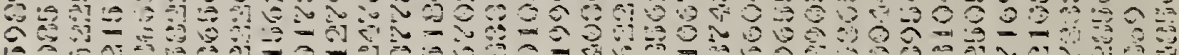

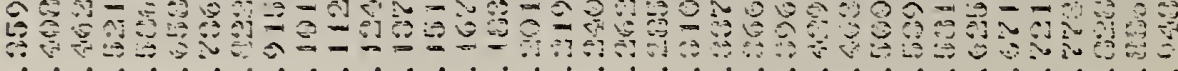

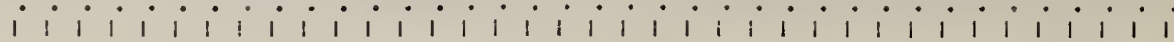

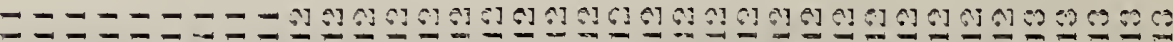

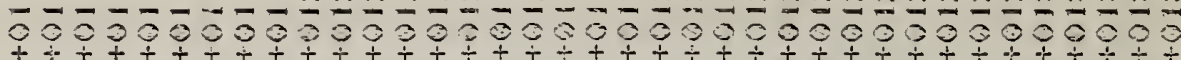

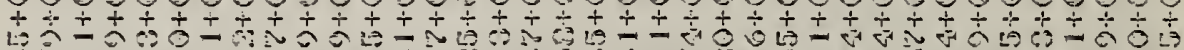
4 C.

$m$ on

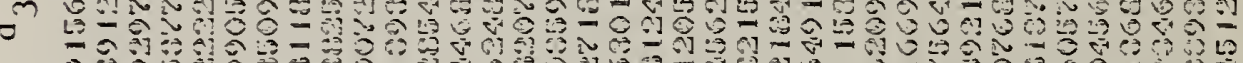

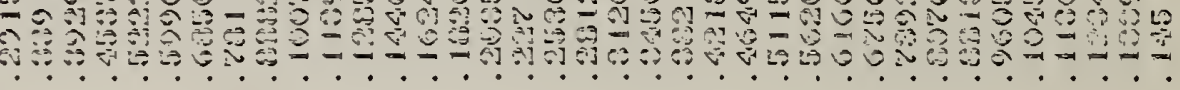

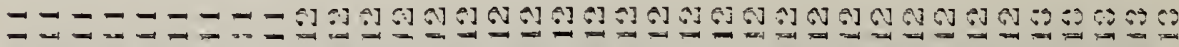

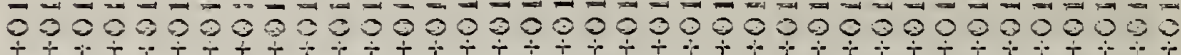
th ङ こ o

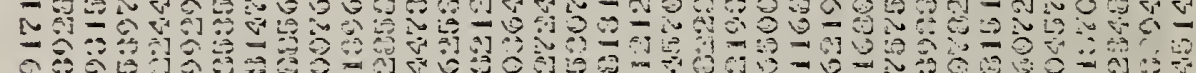

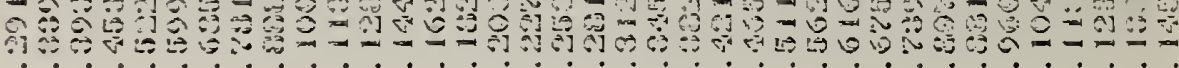
i i i i i i i i i i i i i i i i i i i i i i i

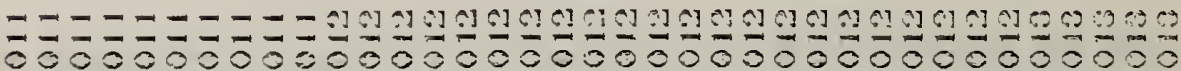
ب.to to

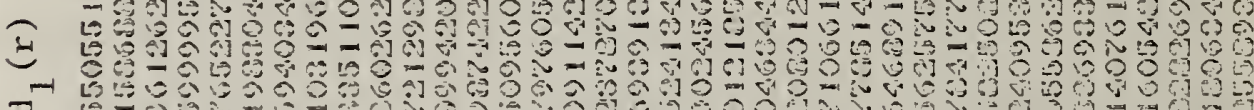
年

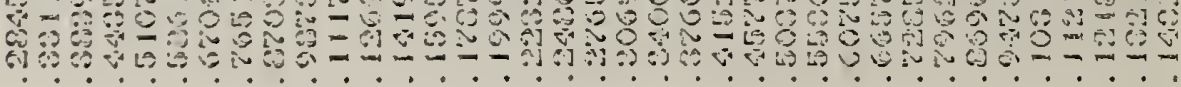

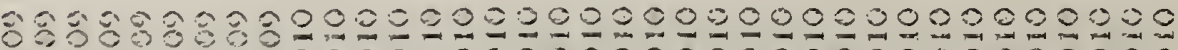

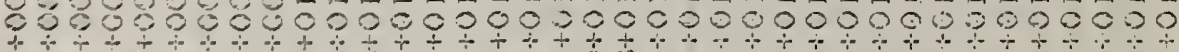
$\exists$ 。 $\tau$ ¿

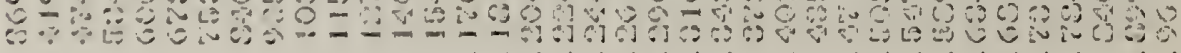
$i+i$ i i i i i i i i i i i i i i i i i i i

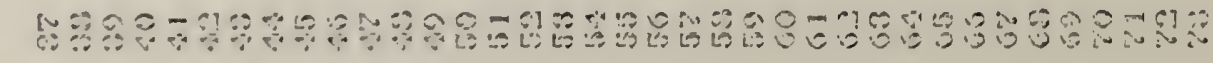



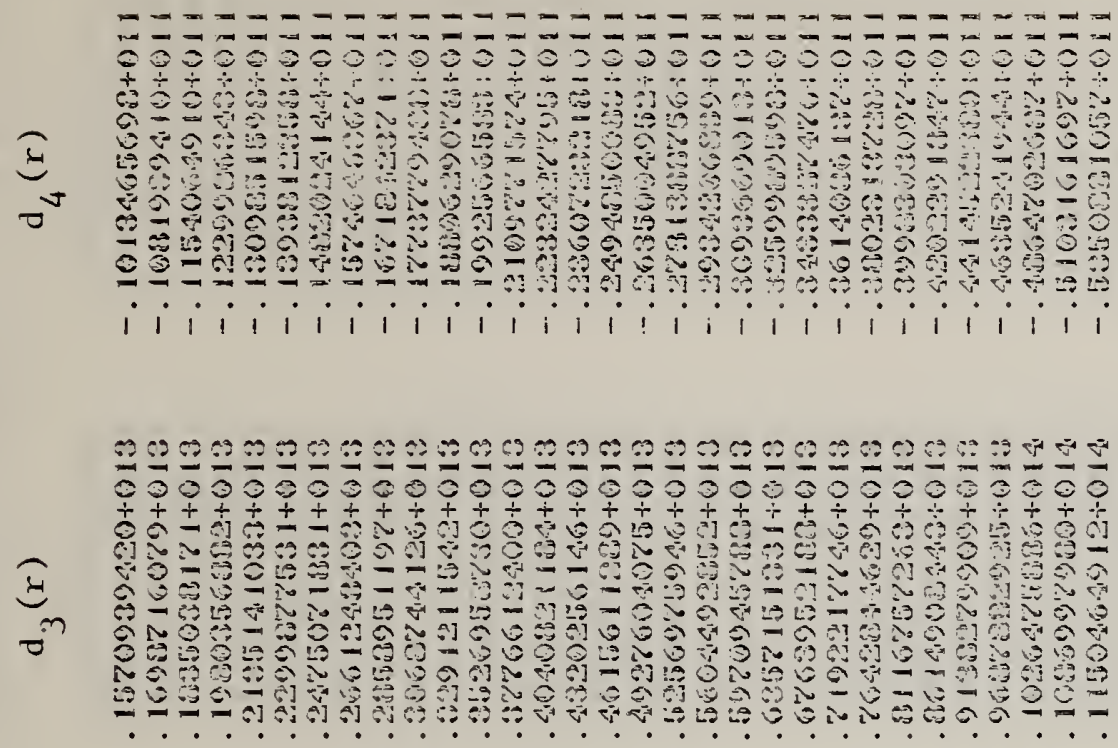

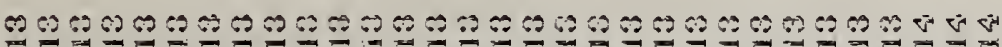

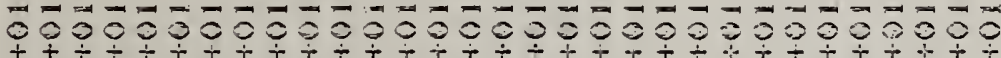

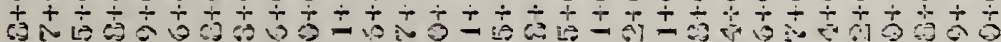

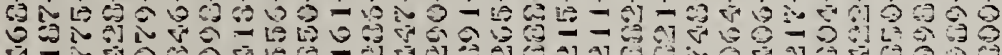

In $\quad$ w

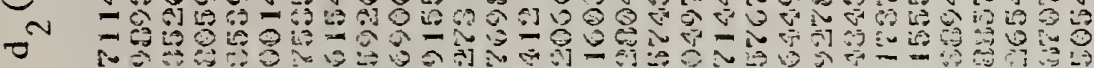

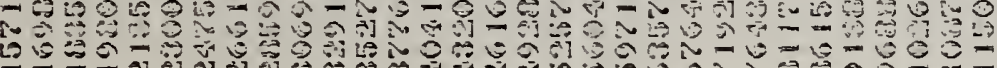
i i i i i i i i i i i i i i i i i i i i i

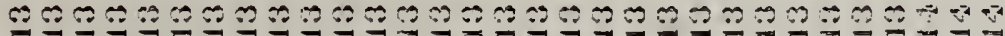

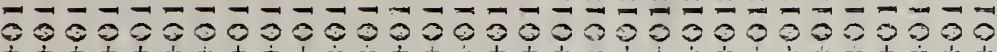

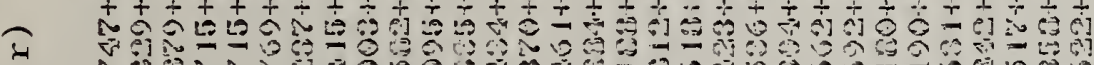
H $\quad \mathrm{d}$

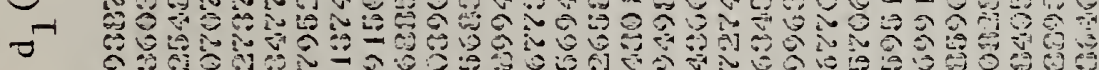
ô

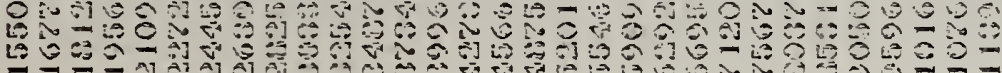

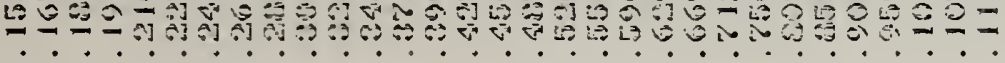

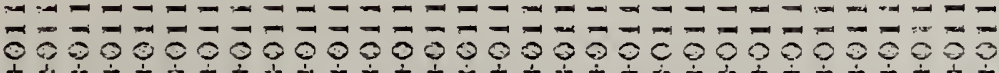

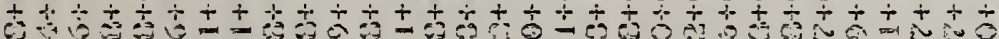

(車 กิ

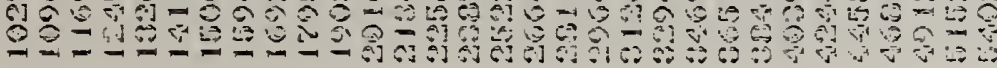

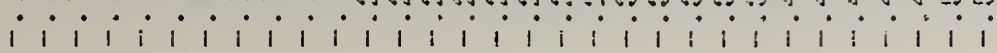

4 


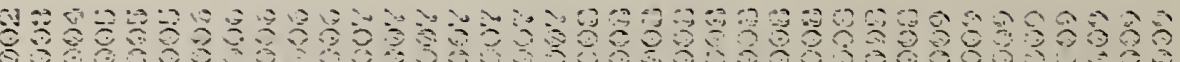

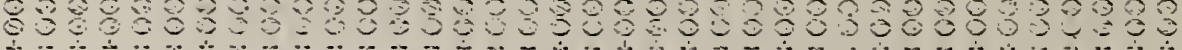

干

$\tau$ -

乞

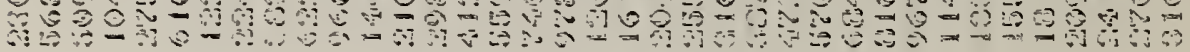

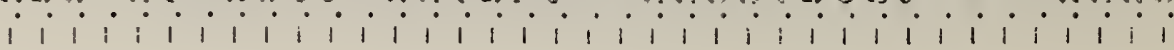

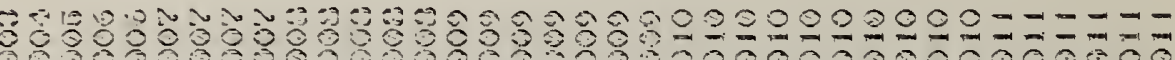

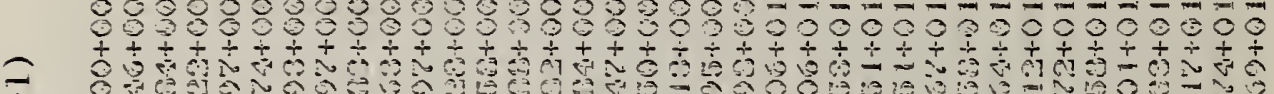

I 80 a

¿ 09090

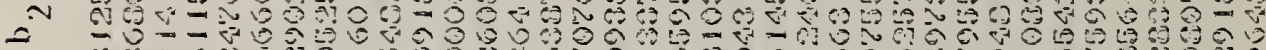
с 告

6. 8.

-

I o o i

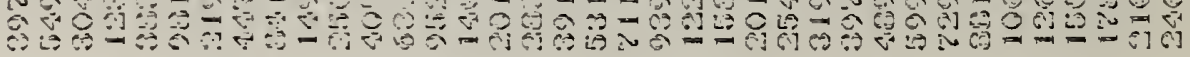

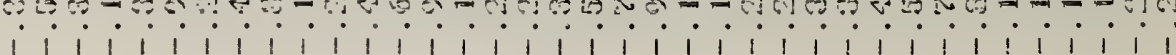

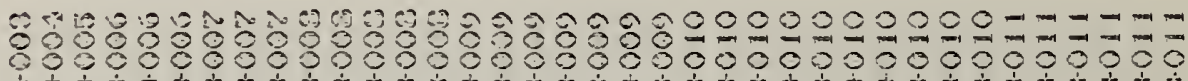

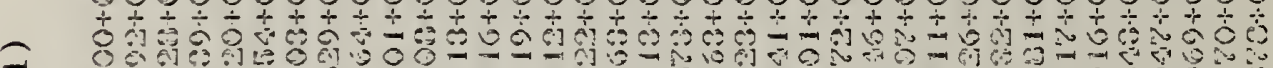

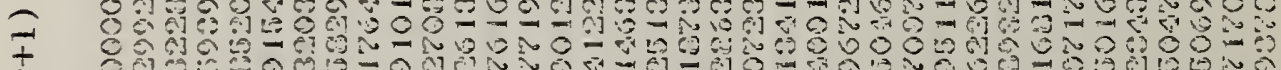

\pm on

o

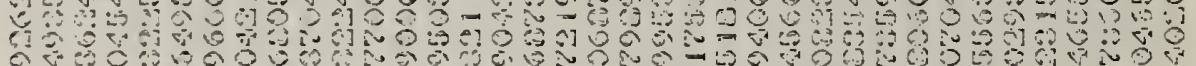
等

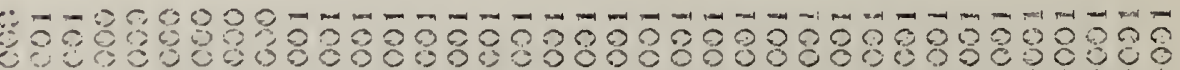

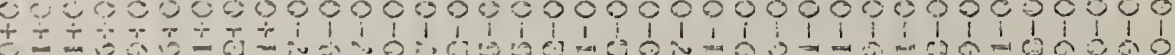

I

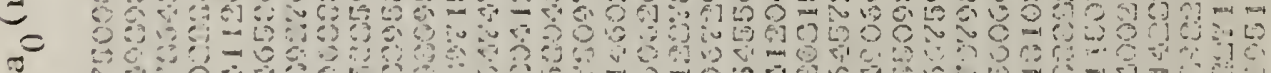
A 0 -

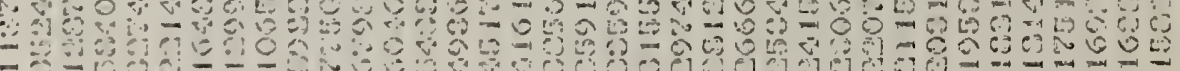
$\because \div-3$.

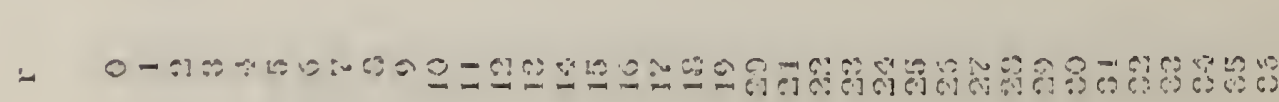




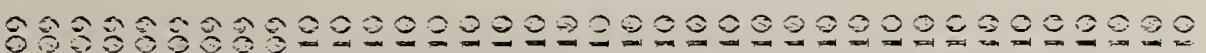

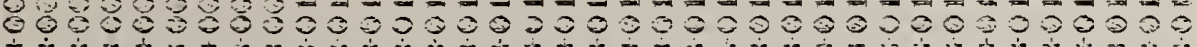

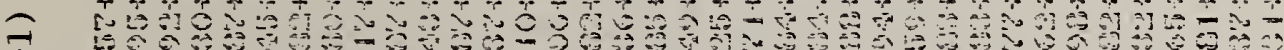
I

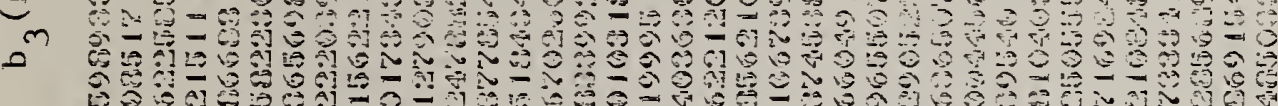

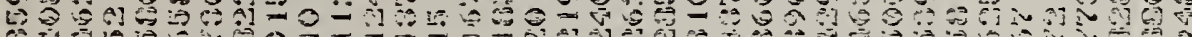
?

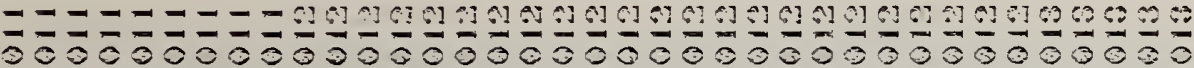

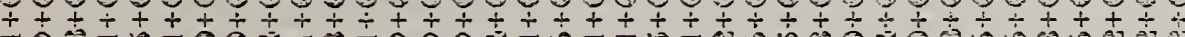

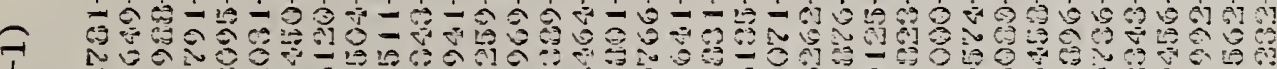

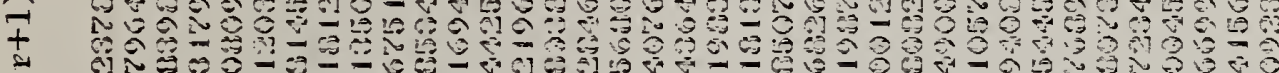
\& N

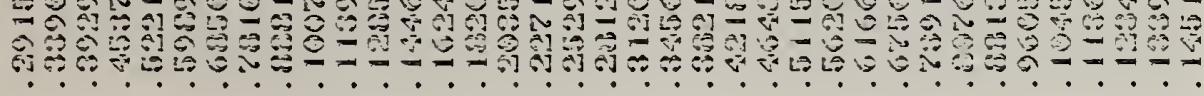

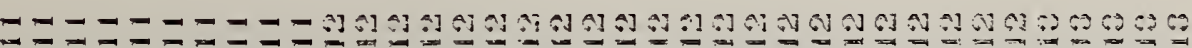

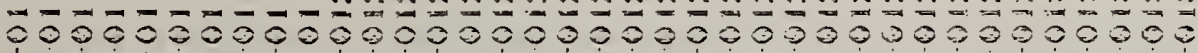

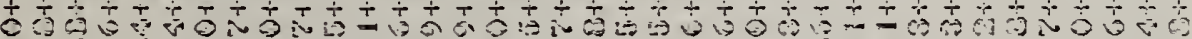

I 100

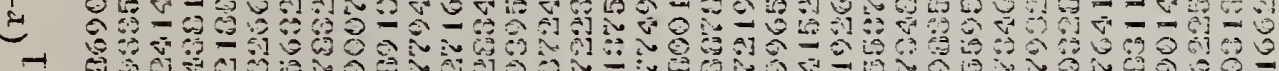

- 4 (

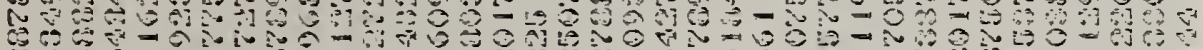

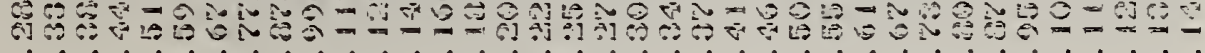

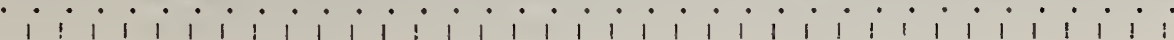

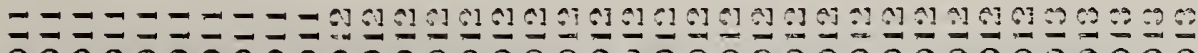

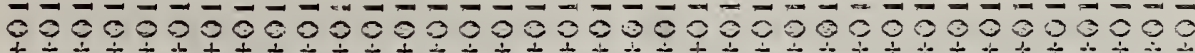

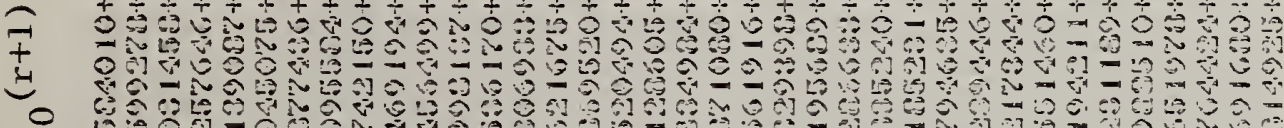
o L

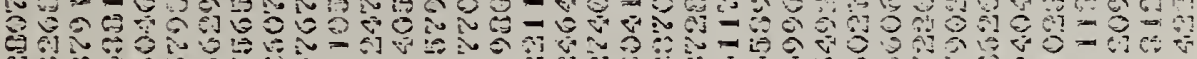

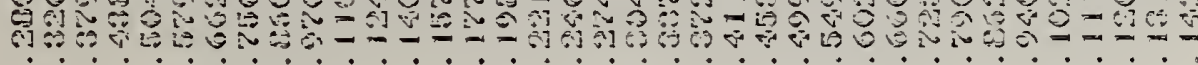

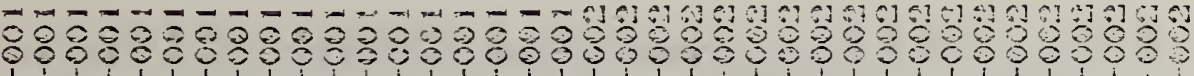

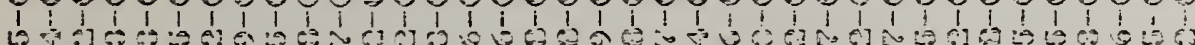

(1) 0100

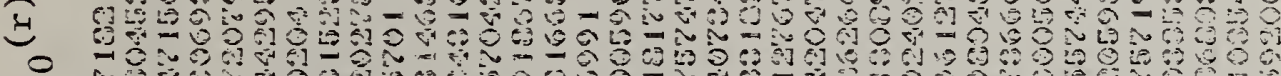

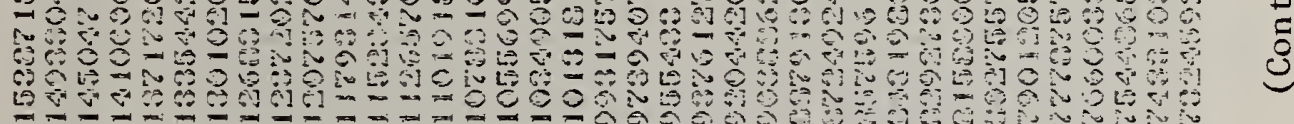

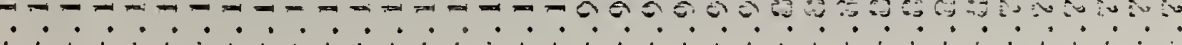

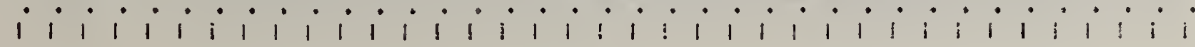

- 


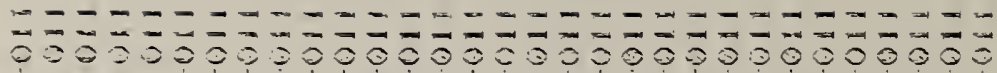
† I ๖

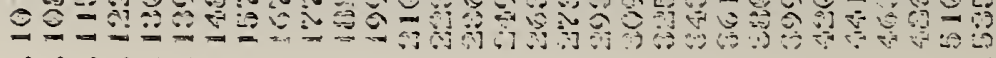

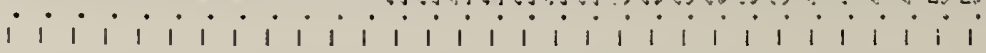

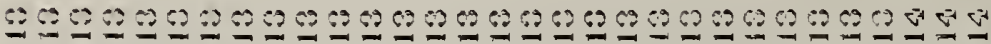

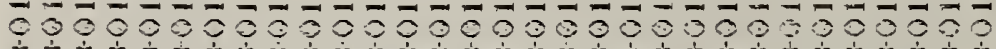
I ง

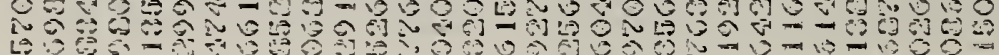

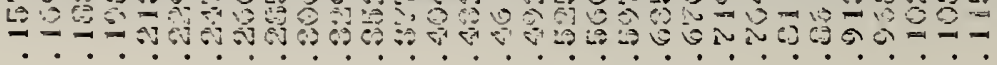

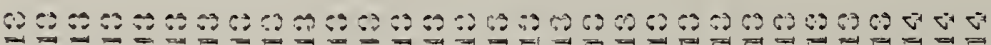

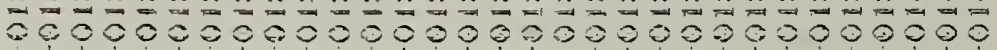

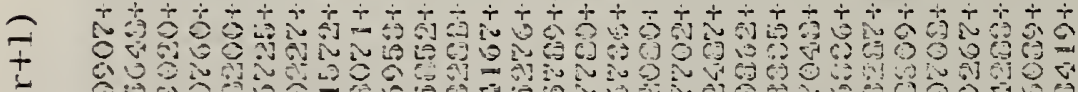
- N -

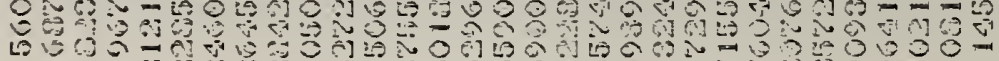
L5 ii i i i i i i i i i i i i i i i i i i i

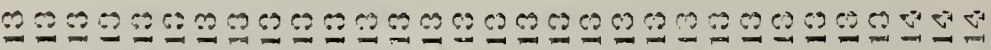
दे

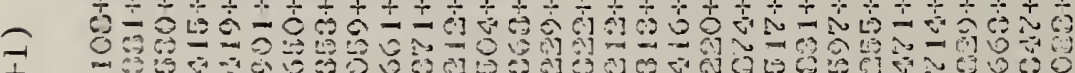
I

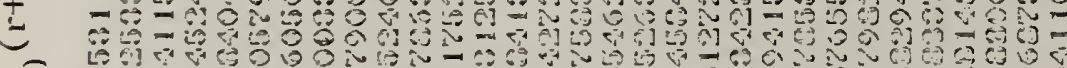
$=$ ○ 40

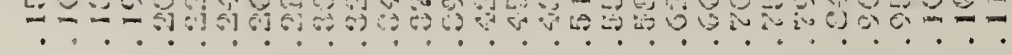

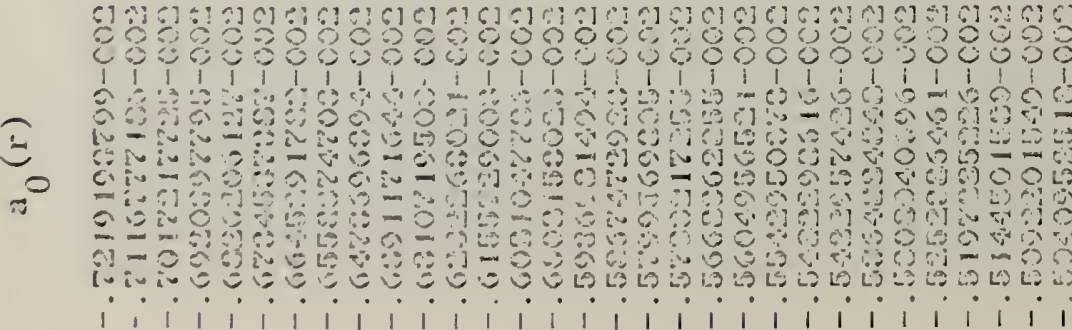

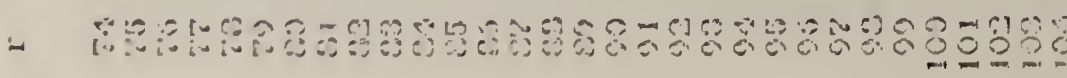




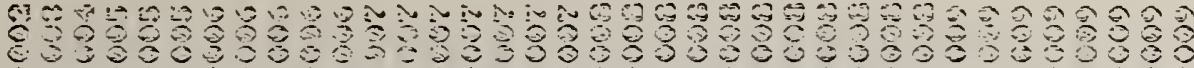

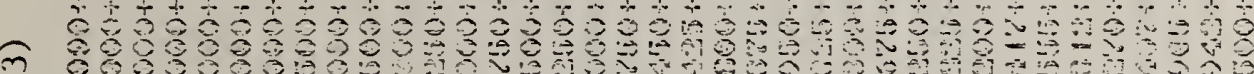

+ + 90909

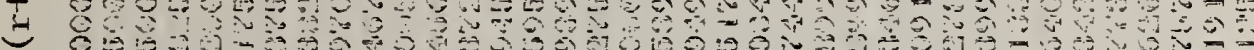
6.

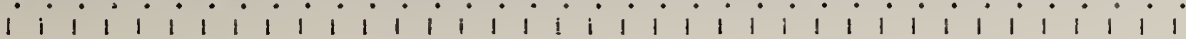

904

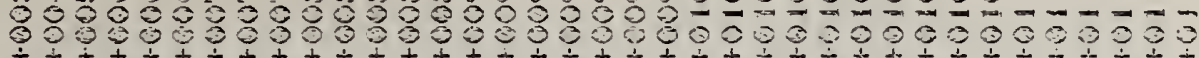

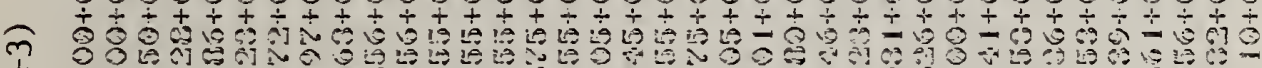

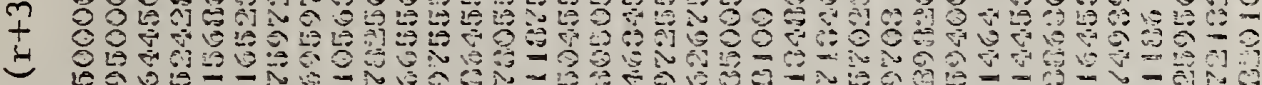

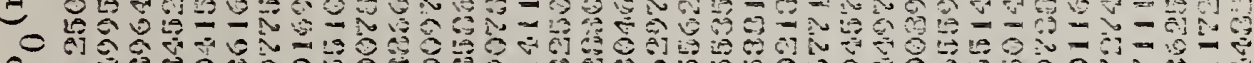
mถัต

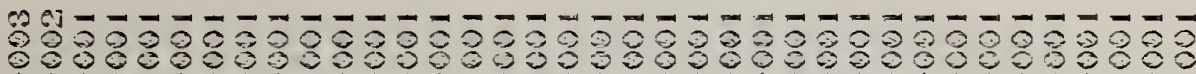

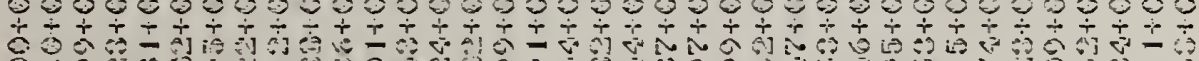

I 89040

N 9809630

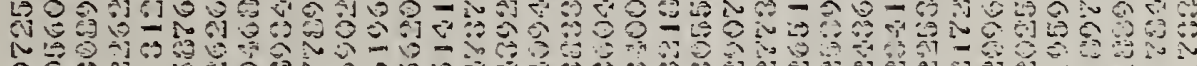

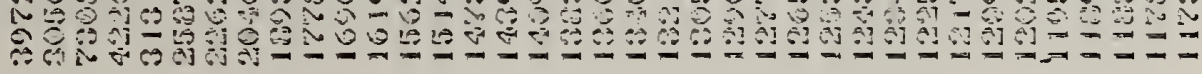

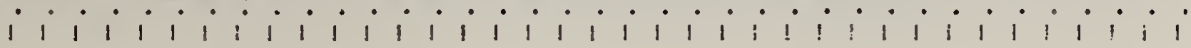

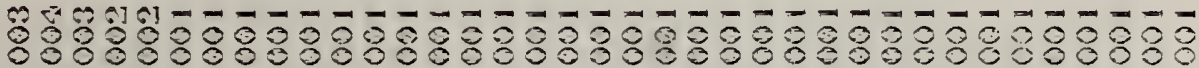

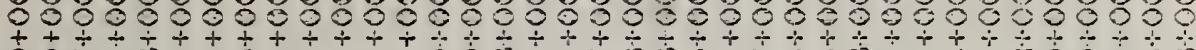

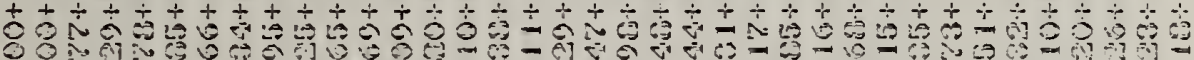

I 8050

क

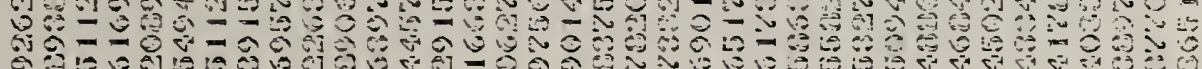

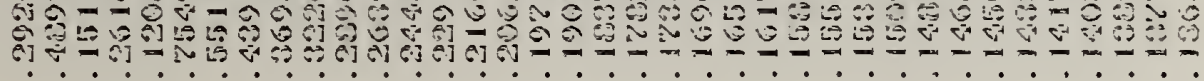

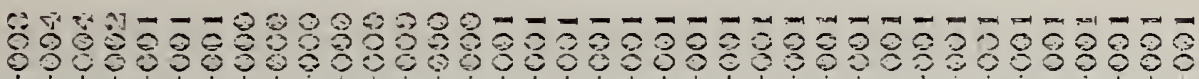

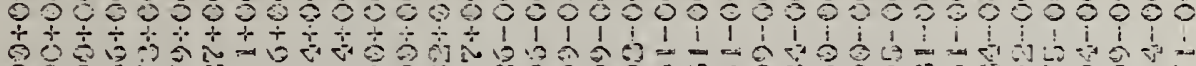

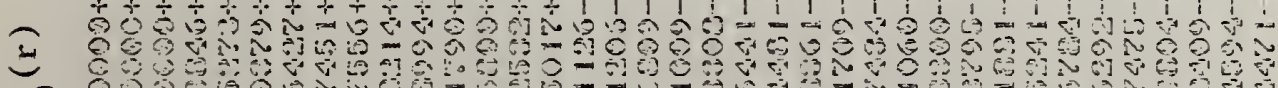

0900 Loth

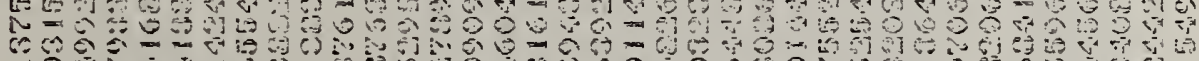

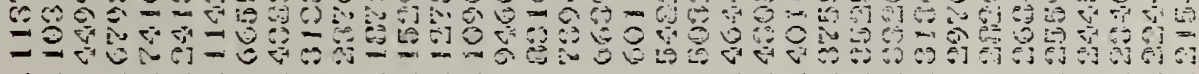
i i i i i i i i i i i i i i i i i i i i i

เ

if

$x^{2}$

ฮี

!I

$\tilde{\Xi}$

3

ส

(1)

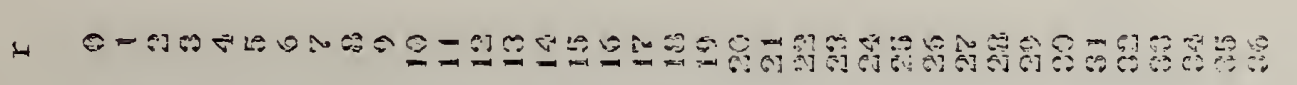


今.

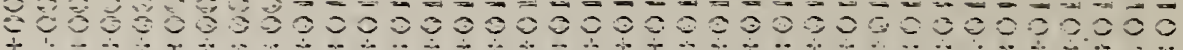

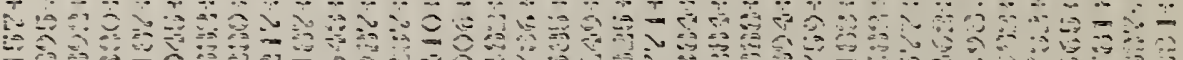

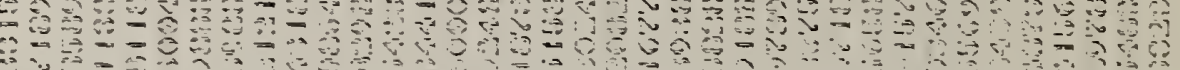
6

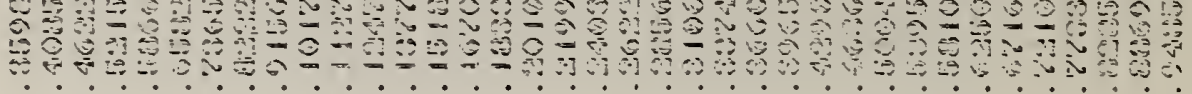

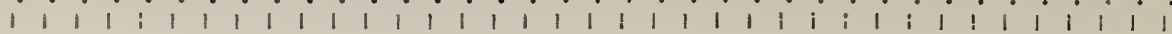

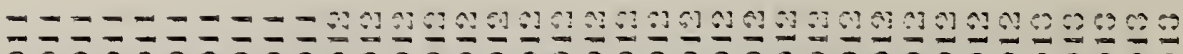
o

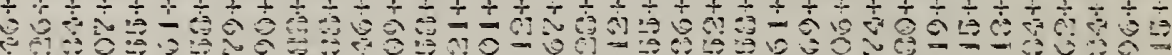
o

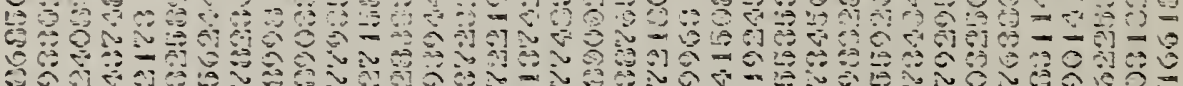

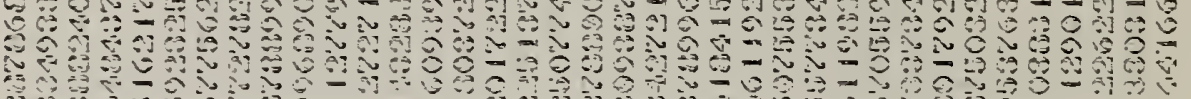

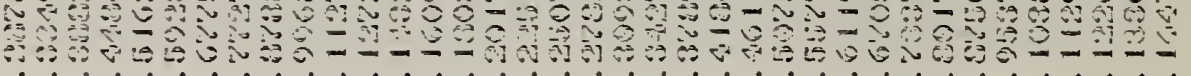

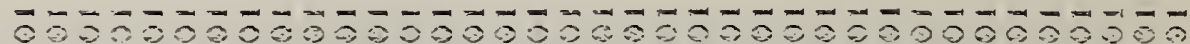

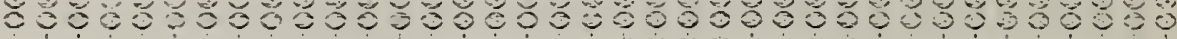
I ศ \% $^{2}$

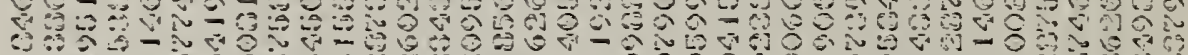

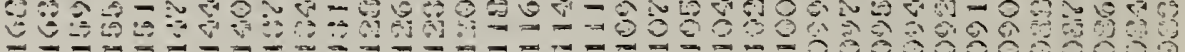

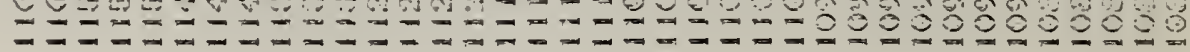
iiiiiiiiiiiiiiliiiiliiiliiiii

б-

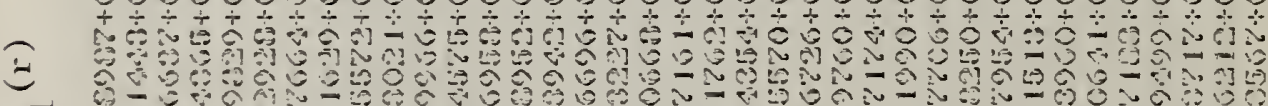

- 120

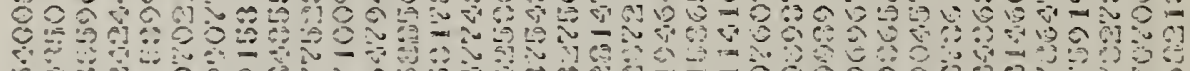

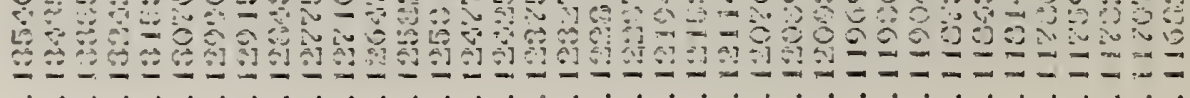

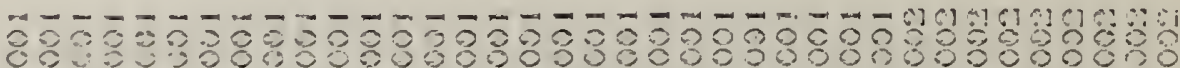

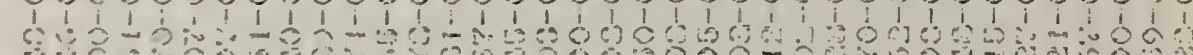

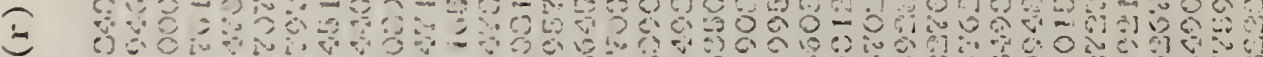

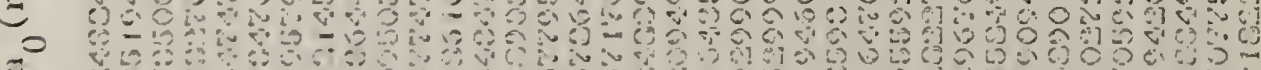

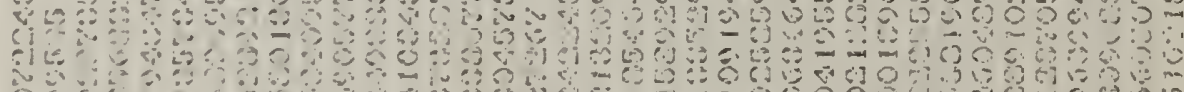

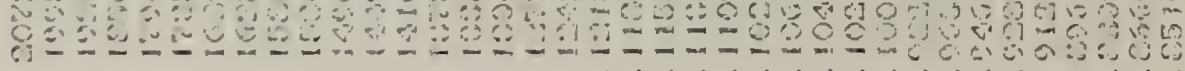
iiliiiiiiiiiiiiiiiilii i iliiii

- 

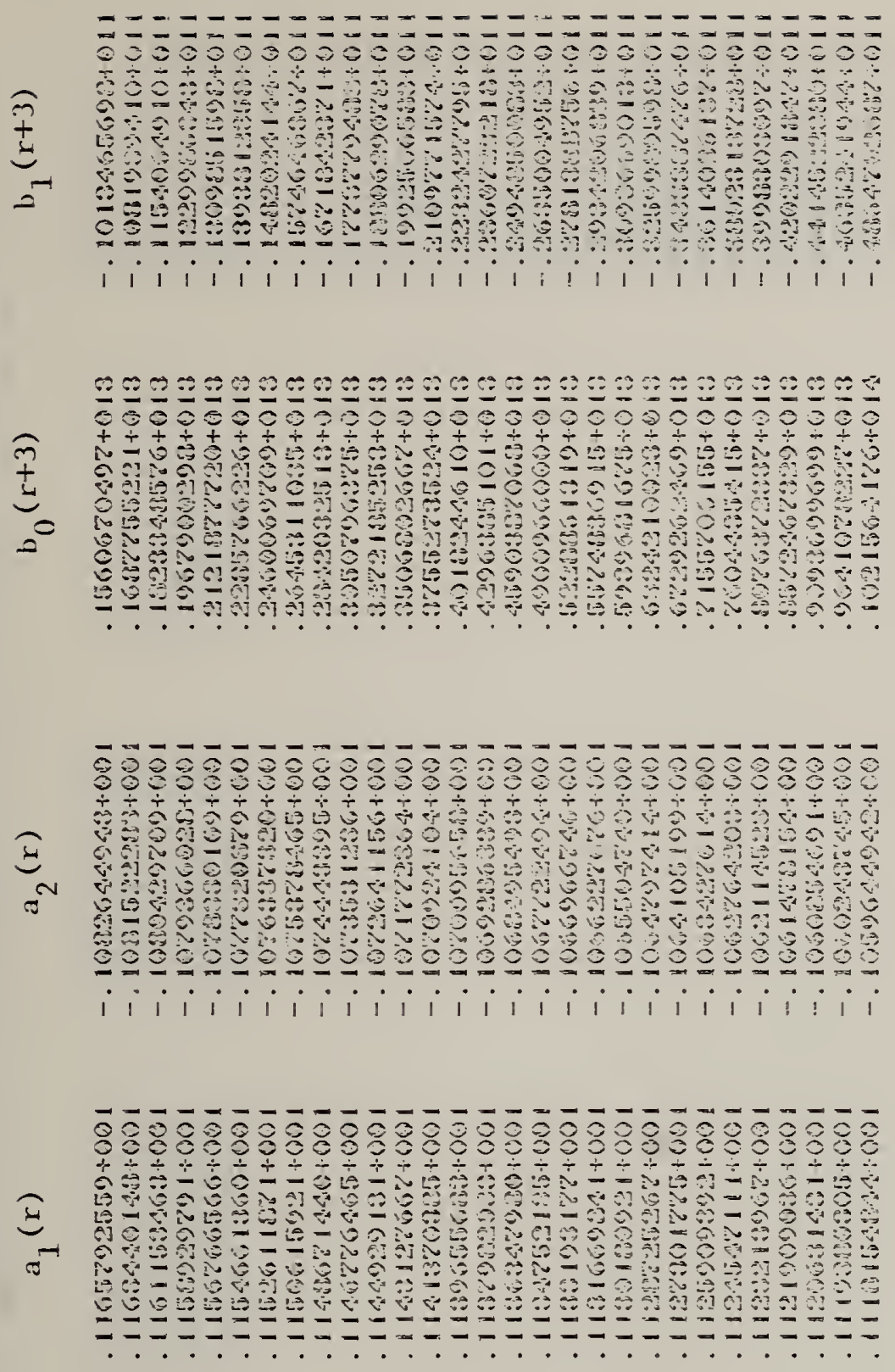

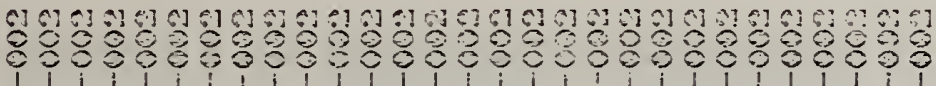

(1)

\& \%

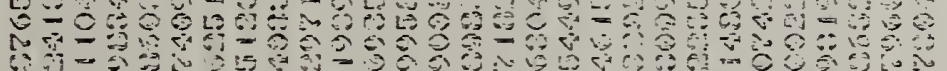

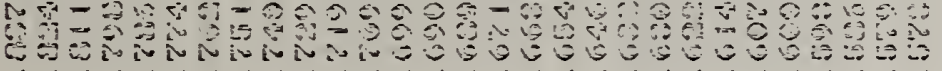
i i i i i i i i i i i i i i i i i

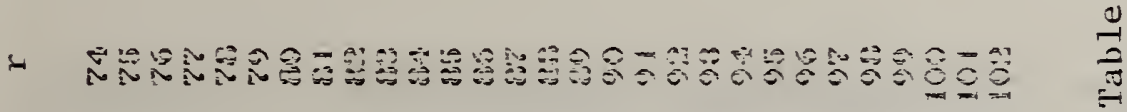



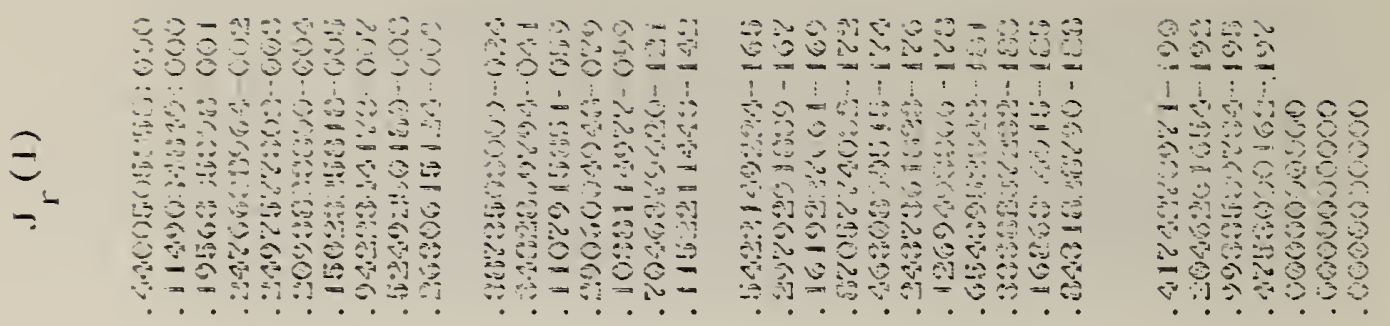

$\underset{\Xi}{\Xi}$
$=-$
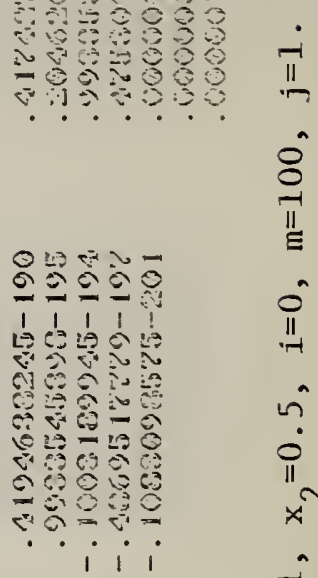

$\underset{\Xi}{\Xi}$

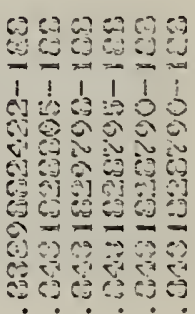

$\overbrace{.1}^{\circ}$

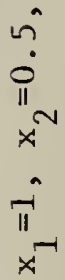

$\underbrace{2}_{3}$

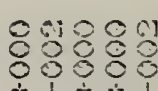

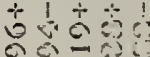
ก a. 650

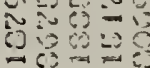

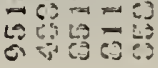
- 1309

11
$=$
$=$
-1
3
5
0
0
0
0
0
0
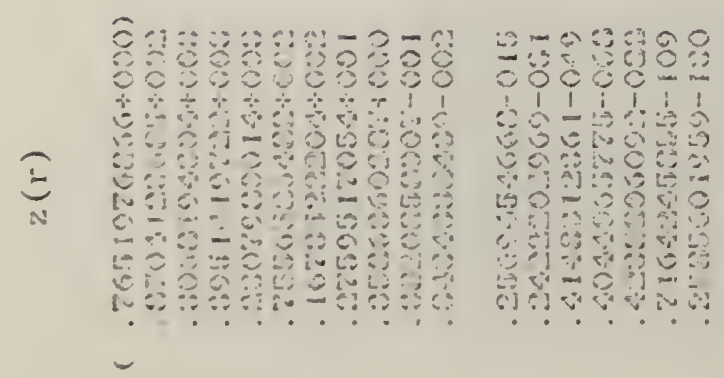

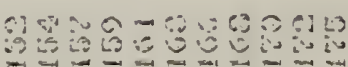

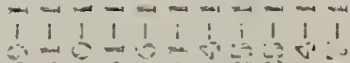
पद

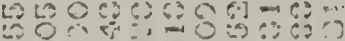

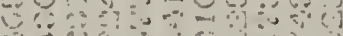

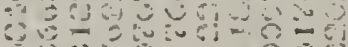
$3-3=-04$

$\%-645=?$

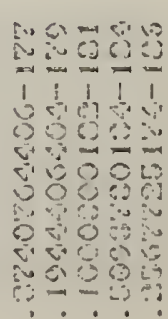

$\stackrel{\square}{\longrightarrow}$

$\stackrel{\varrho}{ \pm}$

:

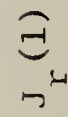

$\because \div-\div ?$

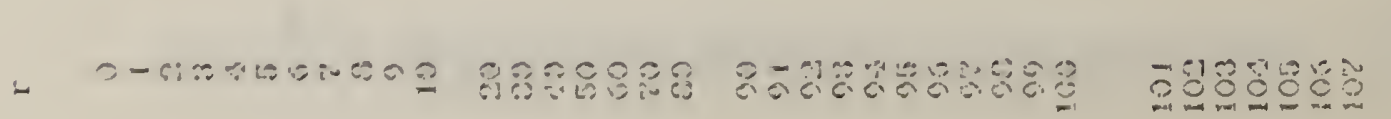

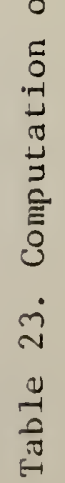




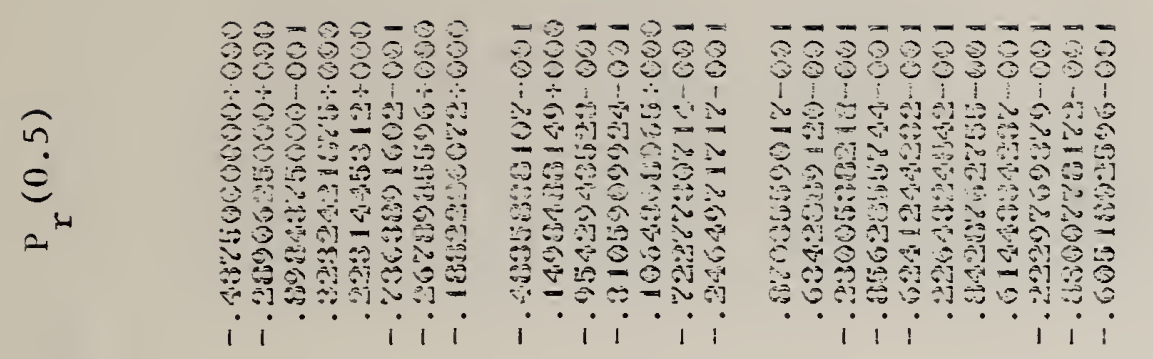

$8 \overline{8}$

ton

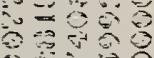

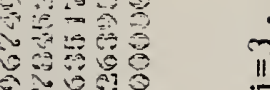

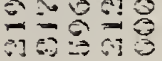

$\because \div$

$\stackrel{\circ}{11}$

$\underbrace{0}_{-1}$

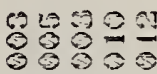

$\begin{array}{ccccc}1 & 1 & 1 & 1 \\ 0 & 0 & 0 & 0 & 1\end{array}$

8 的时

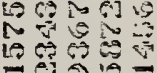

马)

娄

6! $\div i$

II

$\prod_{.1}^{\infty}$

ก.

${ }^{i}$

$37 \overline{8}$

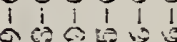

-

80 .

요요의

i

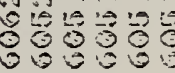

i i i i i

17.

2

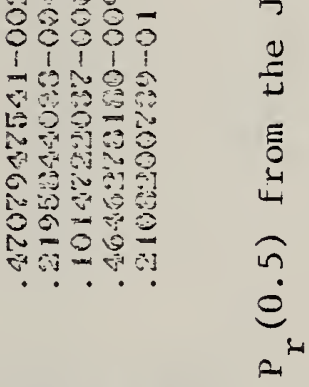

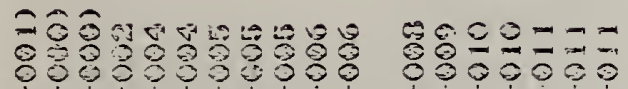

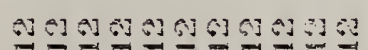

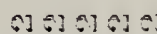

4

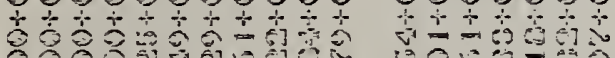

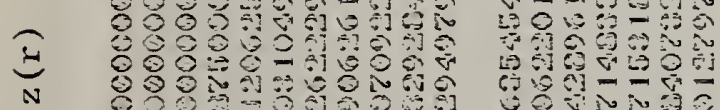

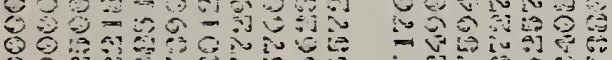
6.

- $\div \div$ is

$i, i \cdots i$

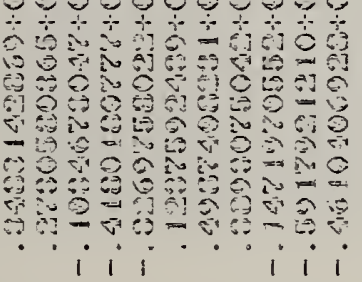

$\frac{0}{6}=\frac{1}{9}=\frac{9}{9}$

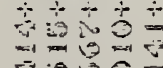

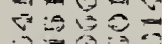

$P=0$

制 8

$\because 6$

$\because$

$x^{x-1}$

il

$\stackrel{1=1}{3}$

둥

矛

ए

$\underset{7}{2}$

$\stackrel{Ð}{\nu}$

E్

ลก

0

$a^{2}$

111

ڤี

$\dot{\Sigma}$

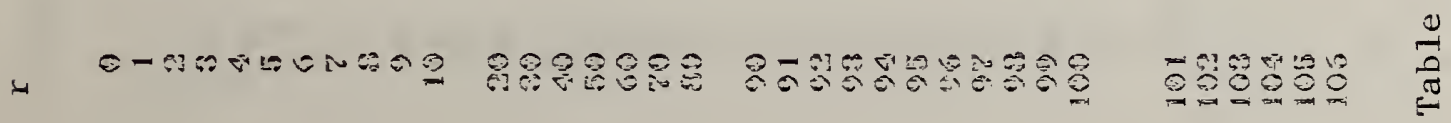



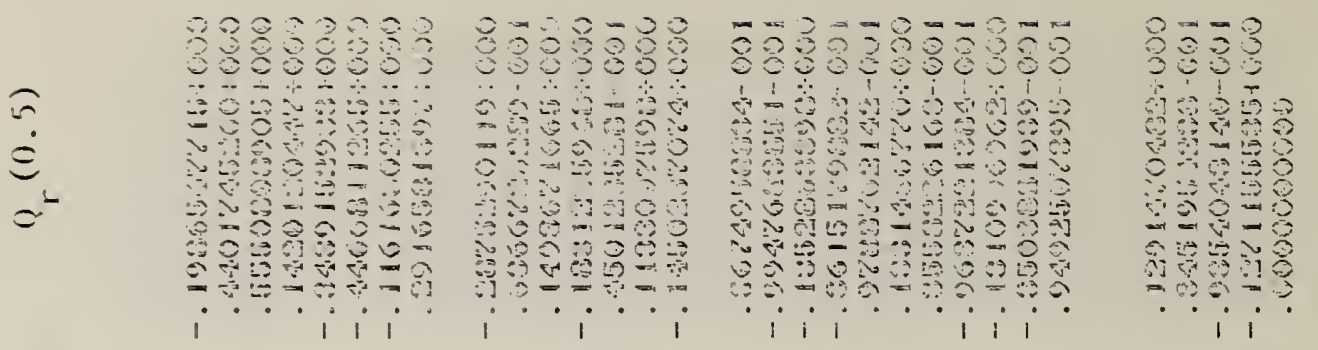

$\bar{E}$

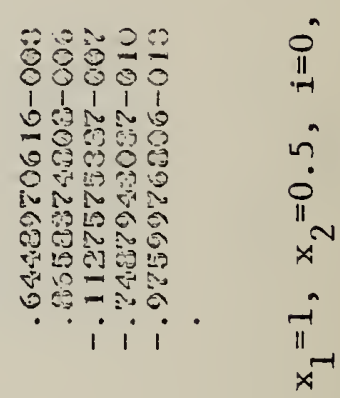

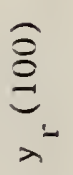

$08 \overline{8} \overline{8}$

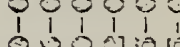
eำ

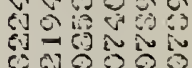

0 in

a

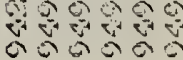

$\dot{n}$

0.00900

II

$\underset{3}{3}$

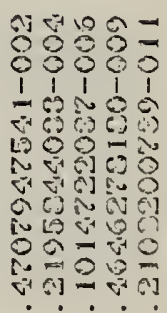

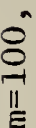

ลิ50ำ t tr

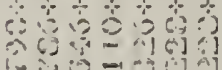
与 $-11 i=0$

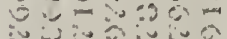
- 01010

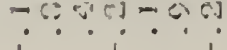

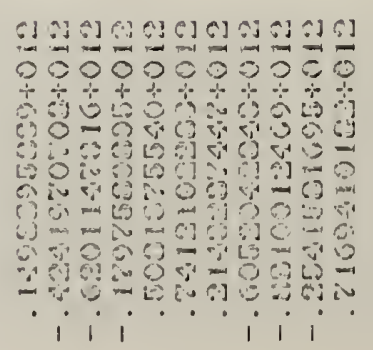

20000 it to ic $=630$

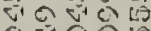
ite?口 000 $0 \mathrm{sin}$ $+?$ 5
0
3
5
0
$0-1$
0
0
0
0

$\stackrel{2}{\check{\zeta}}$ $\stackrel{9}{\perp}$

芩

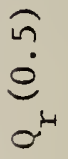

$\overleftrightarrow{4}$

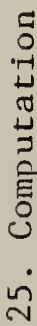

-

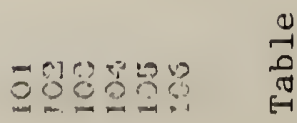



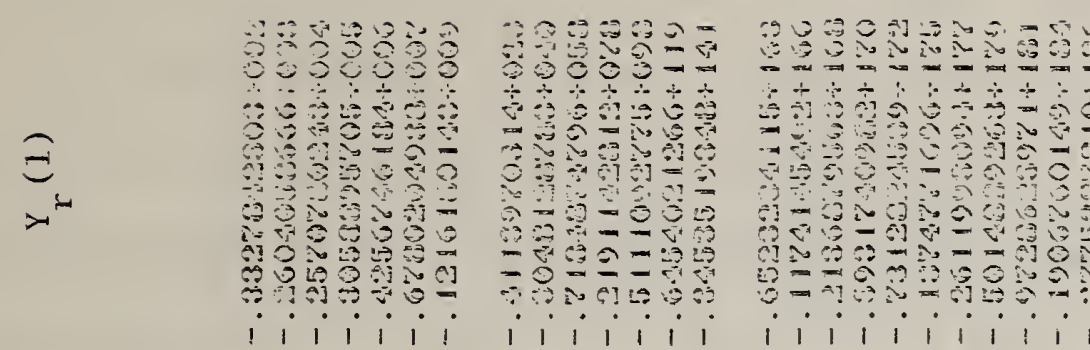

$\underset{+1}{4}$

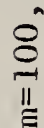

$\overbrace{. \rightarrow-1}^{n}$

$0^{n}$

-11

î

$\frac{1}{3}$

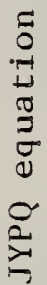

$\stackrel{\cup}{ \pm}$

हొ

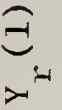

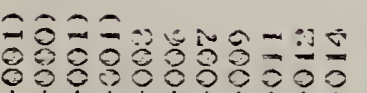

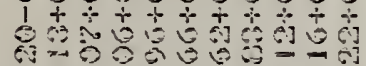

iิ
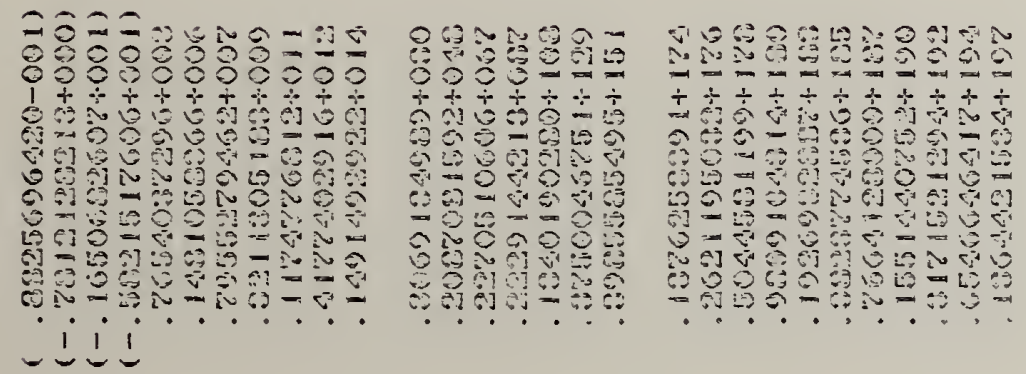

4

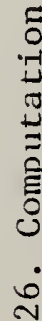

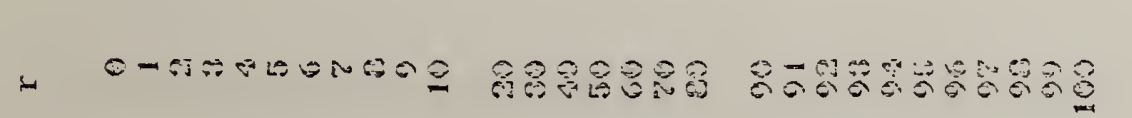

$\underbrace{\infty}_{-10}$ 
Example 4.3.2 In this example we compute the solutions $P_{r}^{1}(0.5)$ and $Q_{r}^{1}(0.5)$ of the homogeneous equation defined in Figure 7 , with $\mu=1$, $x_{1}=1, x_{2}=0.5$, for $r=0,1,2, \ldots, 100$. The algorithm will be stable provided that we take three initial values for each of these functions; compare (4.3.3). Tables 27 and 28 give the results of these computations for selected values of $r$; see $\$ 4.0$ for a full description of these tables. Initial values were obtained by hand calculation of the explicit elementary forms given in Tables 8.2 and 8.4 of [16]. The computed function values agreed satisfactorily for $r \leq 10$ with the limited data available in [16] and [17]. In addition, satisfactory agreement was obtained with double-precision values produced by an unpublished program of Dr. J. M. Smith; compare Example 4.3.1. Consequently, the stability of the method is confirmed for this example. 

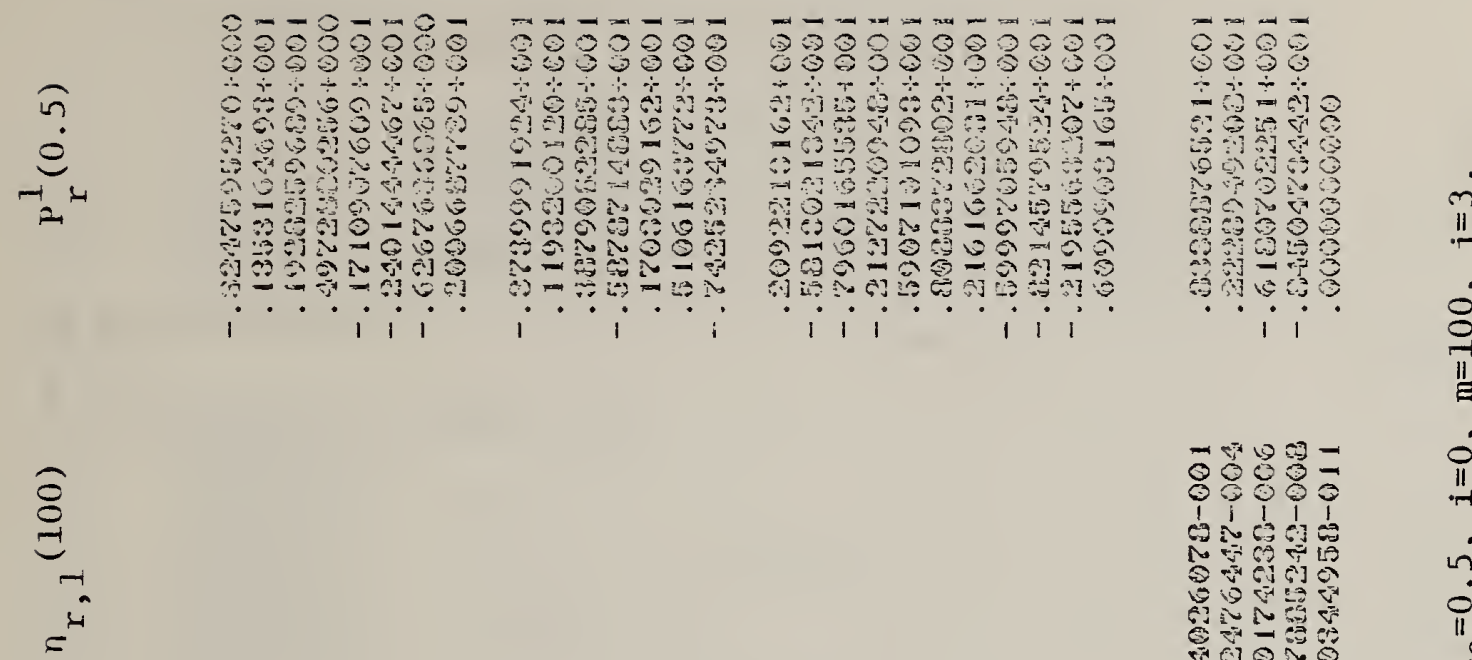

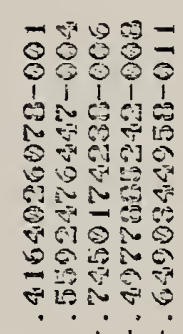

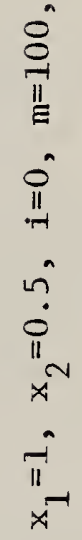

$\underbrace{\varnothing}_{n}$

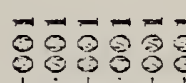
t它t施市 os ivo

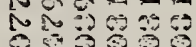
6 95

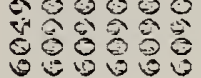

in 굴

$\underbrace{4}_{3}$

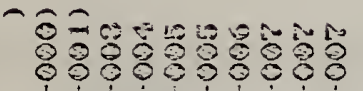

20ำำ

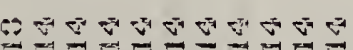

$\pi i \frac{0}{0}$

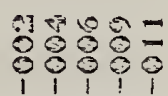

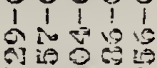

No 0 :

a 610

से

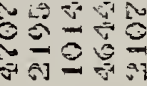

2000\%०0000 0604060 $6060 \mathrm{\sigma}=0$

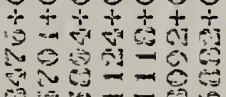

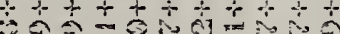

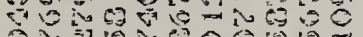

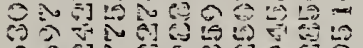

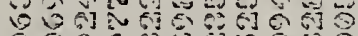
- 040996

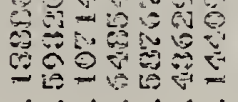
$60 \% 0600=0$

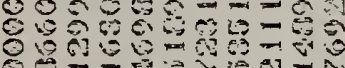
$80-1 i=0$ $\sim i$ i 606 ${ }_{i} i, \cdots, i i$

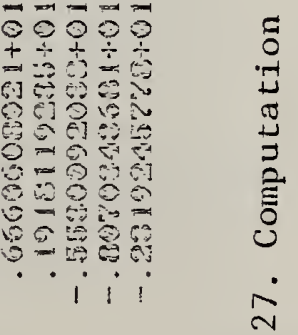

म

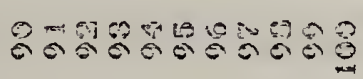



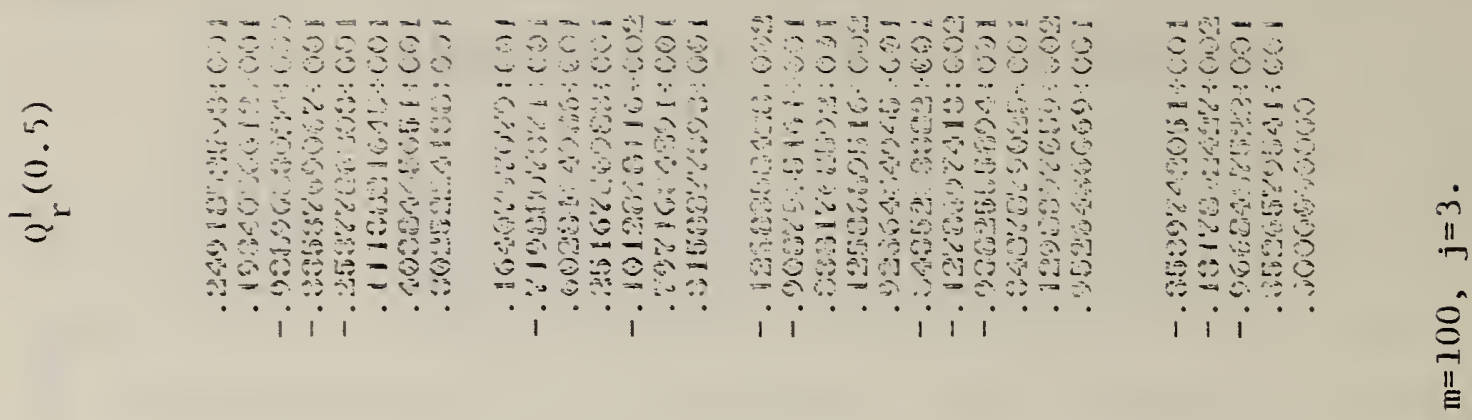

$\overline{\bar{y}}$

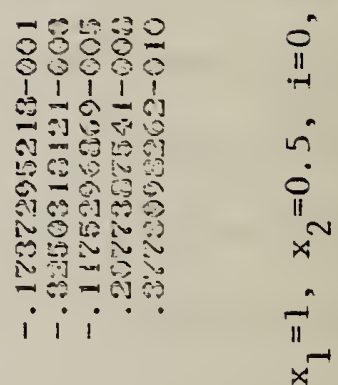

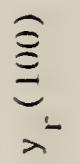

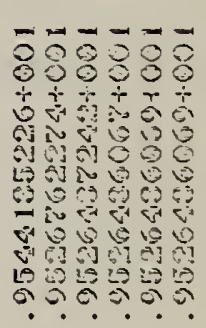

201

$\underset{\Xi}{\Xi}$

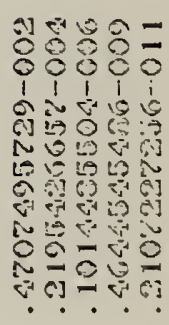

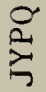

0080

a 20 s

岌

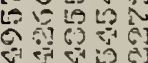

0

.

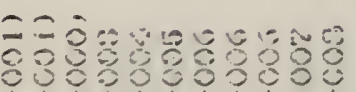
$+4+4$

¿

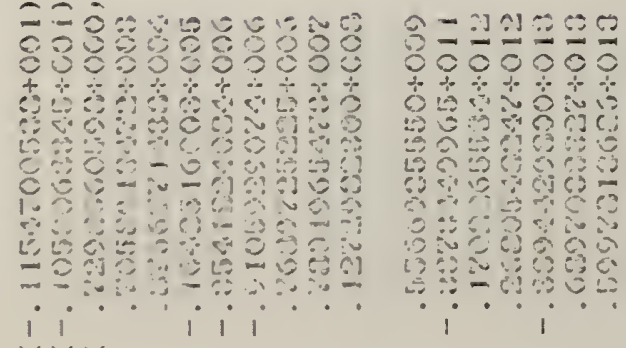

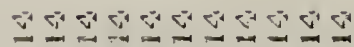

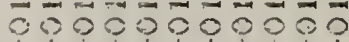
10

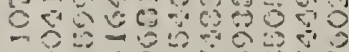
a be -

80006 $\because 6 \div-34 \div: 1$ $i i \cdots$

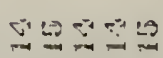
웅요 $8 \div-6$ O4 5 क⿺辶 के $\Rightarrow 5$ उदि 政 $\therefore i$. . ह 苘 4 苛 $\stackrel{\infty}{v}$ 


\subsection{Examples Involving the Inhomogeneous JYPQ Equation}

Using Stirling's formula, we find

$(4.4 .1) \quad \frac{\Gamma(r+\mu+1)}{\Gamma(r+3 / 2)} \sim r^{\mu-1 / 2}, r \rightarrow \infty$

for fixed values of $\mu=0,1,2, \ldots$. Comparison of (4.2.1) with (4.3.1), $(4.3 .2)$ and $(4.4 .1)$ shows that

$$
E_{r}\left(x_{1}\right)<\left\{\begin{array}{c}
P_{r}^{\mu}\left(x_{2}\right) \\
Q_{r}^{\mu}\left(x_{2}\right)
\end{array}\right\}, \quad r \rightarrow \infty
$$

for fixed $\mu, x_{1}$ and $x_{2}$ such that $x_{1}>0$ and $x_{2}<1$. Accordingly, the Anger-Weber function is a solution of type $I$ and asymptotically the correct number of initial values to be used in every case is $j=i$; compared (4.3.3). However, the separation of $E_{r}\left(x_{1}\right)$ from the solutions of type 2 is weak when $\mu=0$ (being of relative order $r^{-1 / 2}$ at the odd terms of the sequence and $r^{-3 / 2}$ at the even terms of the sequence). The separation is also weak when $\mu=1$ (being of relative order $r^{-3 / 2}$ at the odd terms of the sequence and $r^{-5 / 2}$ at the even terms of the sequence). Accordingly, we treat $E_{r}(1)$ as a solution of type 2 in both cases, even though when $\mu=1$ greater numerical instability is incurred than in any of the other examples. This is especially true at the even terms of the sequence, which are separated from the Legendre functions by the relative order of $r^{-5 / 2}$.

From $(4.2 .4)$ and $(4.3 .3)$ it is seen that the type of the Struve function $\mathrm{H}_{r}\left(\mathrm{x}_{1}\right)$ with respect to the JYPQ operator is 0 for all admissible values of $x_{1}$ and $x_{2}$. But again because of the weakness of the separation, we regard $\mathrm{H}_{r}\left(\mathrm{x}_{1}\right)$ as belonging to type 1 . 
Example 4.4.1 For the inhomogeneous fourth-order difference equations defined in Figure 7 , we compute $E_{r}(1)$ with $\mu=1, x_{1}=1$ and $x_{2}=0.5$, For $r=0,1,2, \ldots, 100$; and $H_{r}(0.1)$ with $\mu=1, x_{1}=0.1$ and $x_{2}=0.5$, for $r=0,1,2, \ldots, 50$. (We restrict the sequence of $H_{r}(0.1)$ to $r \leq 50$ for the same reasons that were described in Example 4.2.1.) For reasons given above, we use 3 initial values (rather than 1 ) for $E_{r}(1)$ and 1 initial value (rather than 0 ) for $\mathrm{H}_{\mathrm{r}}(0.1)$. Initial values for $\mathrm{E}_{\mathrm{r}}(1)$ and $\mathrm{H}_{\mathrm{r}}(\mathrm{O} .1)$ were computed from series expansions given in [21] and checked against values found in [21] and [23].

Tables 29 and 30 give extracts from the results of these computations for selected values of $r$; see $\$ 4.0$ for a full description of these tables. Comparison of the function values produced by the algorithm with values given in [21] and [23] show good agreement over the available ranges, wich, however, do not extend beyond $r=13$. Comparison of the values of $E_{r}(1)$ in Table 2.9 with values computed to 10 significant figures from the series expansion shows a steady deterioration of precision at the even values of $r$, culminating in a relative error of $0.5(10)^{-5}$ at $r=100$. At the odd values of $r$ the precision also deteriorates steadily, but at a slower rate, culminating in a relative error of $0.8(10)^{-7}$ at $r=99$. These rates of loss of precision are consistent with the separation ratios of $r^{-5 / 2}$ and $r^{-3 / 2}$ which govern the growth of the error in this example.

That is, an individual relative rounding error of, say, $10^{-10}$ in one of the stored initial values of $E_{r}(1)$ will be amplified by a factor of approximately $100^{5 / 2}=10^{5}$ at $E_{100}(1)$ and by $100^{3 / 2}=10^{3}$ at $E_{99}(1)$. (As noted in Eq. (4.2.1), the function is in behavior different at even and odd values of $r$. ) These amplifications after 100 steps are greater than the factor of 100 that was predicted and observed in the computation of 
141

$A_{r}(1)$ in Example 4.1 .3 and Table 13, where $A_{r}(1)$ was computed as if it were a function of the next higher type than it actually is.

Finally, comparison of the values of $\mathrm{H}_{\mathrm{r}}(0.1)$ in Table 30 with values computed to 10 significant figures from the series expansion shows agreement to 9 or more significant figures at all values of $r$. The loss of precision in the strive function computation is slight, due to the separation ratio of only $\mathrm{r}^{-1 / 2}$, but it offsets impossibly slow convergence which would be evidenced if the theoretically correct number of initial values were to be taken. 


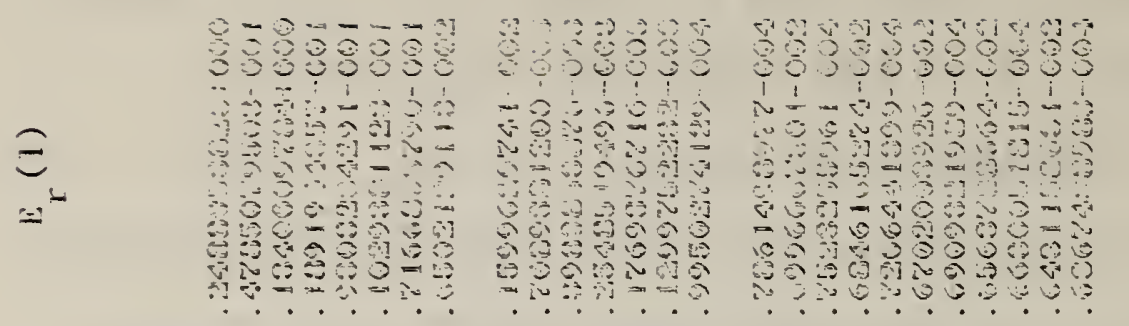

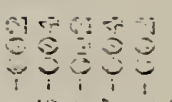

60

7 is

Natos

管

$\stackrel{0}{\tilde{\sigma}}$

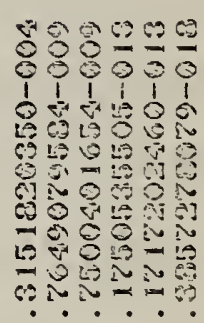

$\stackrel{\circ}{8}$

$\stackrel{8}{8}$

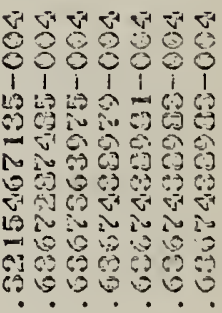

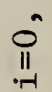

2
3
3

बल. $808=5$

今心

to 60 :

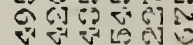

0
$x \rightarrow 4$

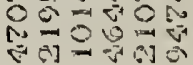

范

. $\because$

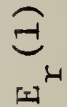

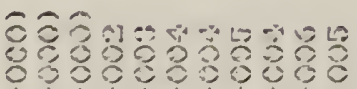

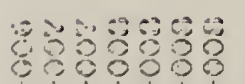

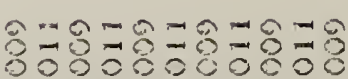

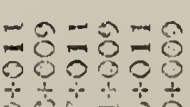

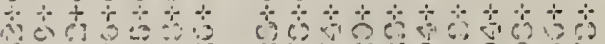

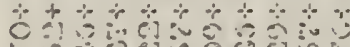

I (1) 6. a 然 $0-100$ ?

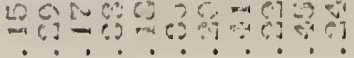
c) 80000

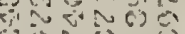

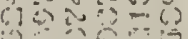
- o 40 4010 2., is 虫

苟 จ่

$-00300000$ 

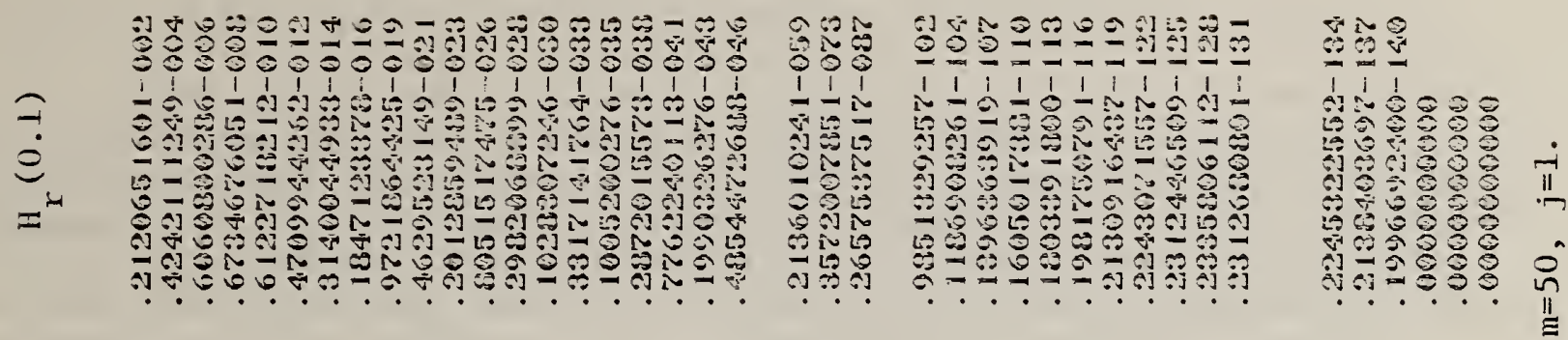

$\underbrace{\widehat{B}}_{-a}$

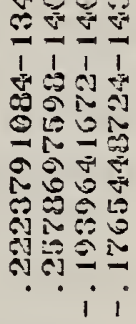

in
in
iI
$\Xi$

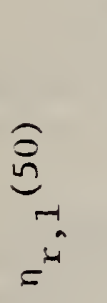

$\underbrace{}_{\substack{0 \\ \text { on }}}$

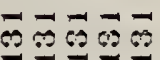

$1+1 \div-1$

बे 800

N.9.9

स्.

ษับำ

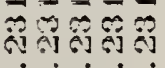

$\overbrace{. \rightarrow-1}^{n}$

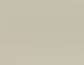

$\underbrace{\substack{3 \\ 0}}_{\substack{\text { th } \\ 3}}$

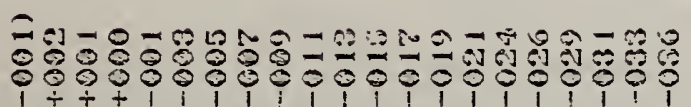

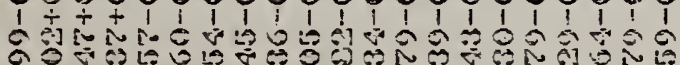

(I

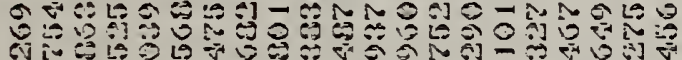

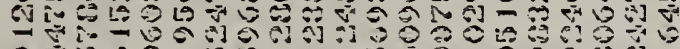

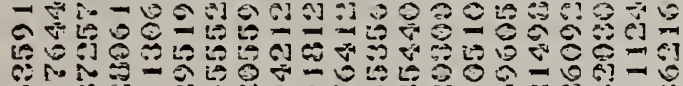

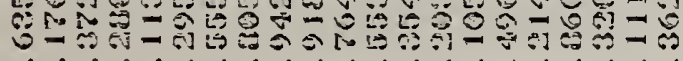

$\sim$

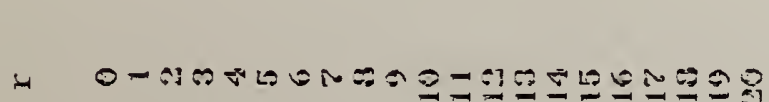

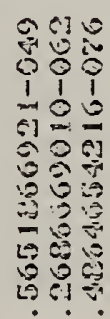

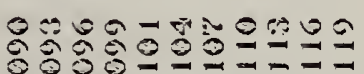

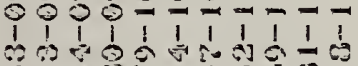

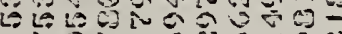

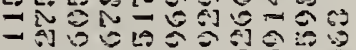

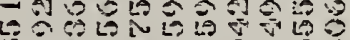
300 -

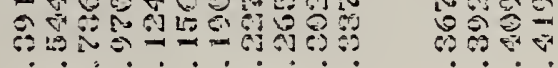

$8 \% 8 \%$

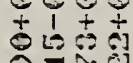
बิำ Nov สำ คิ 象造

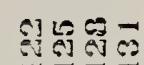

$1 \frac{1}{1}+i$

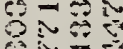

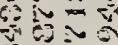

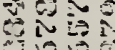

40

$6 \%$

官

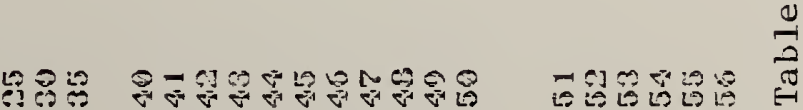


This thesis is concerned with the numerical solution of a broad class of linear difference equations of arbitrary order, and of either homogeneous or inhomogeneous form. An algorithm is presented for the stable computation of any solution of such an equation. The results apply directly to scalar linear difference equations but could be adapted to similar problems posed in vector form.

Historically, the algorithm is an outgrowth of algorithms of Miller and Olver. The underlying idea of Miller's algorithm is the following: If a solution $\mathrm{y}(r)$ has growth behavior, as $r \rightarrow \infty$, that is less than or equal to the growth behavior of all independent solutions, then in the reverse direction its growth behavior is greater than or equal to the growth behavior of all independent solutions. Consequently, because of the superposition principle, errors introduced in computing $y(r)$ by backward recurrence cannot grow faster than $y(r)$ itself, and in this sense the backward recurrence process is stable. However, this is true only in a region in which the solutions actually maintain the growth behavior indicated by their asymptotic forms as $r \rightarrow \infty$. For this reason we call this the asymptotic region; evidently it depends on the asymptotic structure of the given difference equation for large $r$.

Miller's algorithm starts the backward recurrence process at a value of $r$ that is larger than any $r$ for which a valid approximation of $y(r)$ is wanted. Arbitrary starting values are assumed. The computed 
solution is essentially a scalar multiple of $y(r)$ for early values of $r$ when ( $i$ ) the difference equation is homogeneous; ( $i i) y(r$ ) is subdominant compared to every independent solution of the equation. Under these conditions the algorithm is stable and $y(r)$ is obtained from the computed solution for early values of $r$ by a normalization procedure, such as matching the value of the computed solution at $r=0$ with $y(0)$ in order to determine the correct scale factor. Modified normalization procedures are required when the asymptotic behaviors of more than one fundamental solution are similar.

Miller's algorithr involves no forward recurrence, and is stable only for solutions whose growth behavior is less than or equal to the growth behavior of all independent solutions. Olver's algorithm was applied originally to second-order inhomogeneous difference equations, in cases where a particular solution $y(r)$ has growth behavior as $r \rightarrow \infty$ that lies between that of a pair of linearly independent solutions of the homogeneous equation. To construct a stable algorithm, olver posed a boundary value problem having one condition at each end. The initial condition is the value of $y(r)$ at a suitable point $r=i$. The terminal condition is zero, it being anticipated that isolated values of $y(r)$ for large $r$ will not be easily obtained. The resulting boundary value problem is a tri-diagonal linear system of algebraic equations. Olver specified a method for solving this linear system that invoives a forward recurrence stage and a backward recurrence stage. The method is equivalent to Gaussian elimination without pivoting, scaled in a 
particular way. Analysis of the method shows that (i) the forward and backward recurrence stages are stable, at least in ranges beginning at a sufficiently large value of $r$; (ii) the second-order linear difference operator that corresponds to the difference equation is, in effect, factored into the product of two first-order operators. Furthermore Olver derived an infinite series expansion of the truncation error, valid at any point $r$, that is incurred by choosing a particular terminal point for the boundary value problem. This enabled the optimal terminal point to be calculated automatically, in contrast to Miller's algorithm in which the terminal point is guessed and subsequently tested.

Extensions of Olver's algorithm to difference equations of higher order were proposed by Zahar, Oliver and Cash. Criteria for selecting the correct number of initial conditions (and therefore the correct number of terminal conditions, since the total number of conditions must equal the order of the difference equation) were given independently by Zahar and Oliver. Zahar proved convergence of the algorithm under assumed conditions on the solutions of the adjoint equation. Oliver investigated stability by analyzing the factorization of the finite boundary value problem. Cash provided an extension of the series expansion of the truncation error. The work of Zahar, Oliver and Cash, taken together, contains the essential elements of a practicable generalization of Olver's algorithm.

In this thesis Olver's algorithm has been extended and analyzed afresh. The vier was taken that a linear difference operator is an 
infinite upper-triangular band matrix. For a specified initial point i and number of initial conditions $j$, an $(i, j)$-factorization of a linear difference operator $D$ is said to exist if Gaussian elimination without pivoting about the $j-t h$ super-diagonal of $D$, starting at the point $i$ on this diagonal, can be carried out indefinitely. This process produces two lower-order difference operators (infinite band matrices). Their product is, of course, the original operator, and the sum of their orders is the order of the original operator. Furthermore, the solution of any finite boundary-value problem having initial point $i$, number of initial points $j$ and terminal point $n$ may be found by forward recursion with one of these lower-order operators followed backward recursion with the other (Theorem 1.2.2). Procedurally, a finite boundary value problem is solved by the algebraic method of forward elimination without pivoting followed by back substitution; this is accompanied by an analysis of the stability of this process in terms of linear difference operators. Determination of the correct number of initial conditions for stability requires a knowledge of the relative rates of asymptotic growth of solutions of the homogeneous equation as $r \rightarrow \infty$. Since the number of 1 inearly independent homogeneous solutions is equal to the order $\ell$ of the linear difference operator, there cannot be more than \& distinct growth rates as $r \rightarrow \infty$. Cases in which there are exactly 2 distinct growth rates are the easiest to treat. In these cases a basis exists that is linearly ordered by growth rate, and we call the operator totally separable. However, there exist operators of practical 
interest, including constant-coefficient operators for which one or more of the distinct characteristic roots are equal in magnitude, that are not totally separable. Accordingly, a more general class of operators, called separable operators, is introduced (Definition 2.2.1). The solutions of a homogeneous equation that has a separable, but not totally separable, operator exhibit less than $\ell$ distinct rates of growth as $r \rightarrow \infty$. Separable operators are characterized by the existence of certain bases, called optimally ranked. An optimally ranked basis includes $\sigma \leq \ell$ disjoint subsets such that (i) neither of two distinct solutions in the same subset dominates the other as $r \rightarrow \infty$; (ii) any two solutions taken from distinct subsets are such that one dominates the other; (iii) no nonzero linear combination of solutions from one subset is dominated by any solution in the subset. These conditions ensure that separable operators are unambiguously defined in the sense that any two distinct optimally ranked bases have similar structure (Lemma 2.2.1).

The disjoint subsets of an optimally ranked basis are arranged in linear order by increasing rate of growth. All possible solutions of an inhomogeneous difference equation with arbitrary right side are classifiable by comparison with the solutions in an optimally ranked basis of the corresponding homogeneous form. Thus a particular solution $y(r)$ is said to be of type $s$ if $(i) y(r)$ is not dominated as $r \rightarrow \infty$ by any solution in the first s subsets of an optimally ranked basis; (ii) $y(r)$ is dominated by every solution in the remaining $\sigma-s$ subsets. The extreme cases $s=\sigma$ and $s=0$ correspond to particular 
solutions for which forward recurrence and backward recurrence of the given difference equation are stable, respectively. For the intermediate cases $0<s<\sigma$, let $j$ be the number of solutions in the first $s$ subsets. If in addition to being separable the difference operator is $(i, j)$-factorizable for some initial point $i$, analysis of the boundary value problem shows that the equivalent pair of forward and backward recurrence problems (Theorem 1.2.2) are stable in the sense that rounding errors are not propagated more rapidly than the solutions, at least in the asymptotic region belonging to the given problem. Accordingly, the solution $y(r)$ of the boundary value problem is obtained in a stable manner by forward elimination without pivoting followed by back substitution ( $\$ 3.3$ and Theorem 3.3.1). Furthermore, under appropriate conditions it follows that $(i) y(r)$ is uniquely determined by $j$ initial values (Theorem 3.1.1); (ii) the algorithm converges pointwise as the terminal point proceeds to infinity (Theorem 3.2.1).

The numerical examples included in this thesis were chosen so that known asymptotic results could be used in conjunction with the new classification theory for separable operators. This enabled the correct number of initial conditions for any chosen solution to be ascertained immediately. The examples also show that it is sometimes desirable to associate a solution with solutions of the next higher theoretical type. This artificial raising of the type is manifested in the algorithm by the specification of the number of initial conditions appropriate for the higher type. This artifice preserves unique determination 
of the solution and also convergence of the algorithm, but introduces instability. It is employed only when the asymptotic separation between two types is weak, consequently the loss of precision resulting from the instability is mild. The compensating advantage is that the rate of convergence is increased very substantially, enabling a much smaller terminal point to be used.

The trade-off between numerical instability and slow convergence can be regarded heuristically in the following way. First, suppose $u(r) \sim e^{-r}$ and $v(r) \cdots e^{r}$ are two fundamental solutions of a second-order equation. Then one initial value of $u(r)$ determines the function uniquely and the algorithm is both convergent and stable. Furthermore, the rate of convergence is very rapid. This can be seen from the equation

$$
u_{n}(r)=u(r)-\frac{e^{-n}}{e^{n}} \cdot v(r)\{1+o(1)\}, n \rightarrow \infty \text {, }
$$

where $u_{n}(r)$ is the solution of the approximating boundary value problem having $n$ as its terminal point (compare Eq. 3.2.6). The rapid convergence of $u_{n}(r)$ to $u(r)$ as $n \rightarrow \infty$ for fixed $r$ is readily apparent. Thus the algorithm performs very well with regard to both numerical stability and rapidity of convergence when the separation of solutions is strong as in this example of exponential separation.

Next, suppose that $u(r) \sim r^{-1}$ and $v(r) \sim r^{-1 / 2}$. Here again, one initial value of $u(r)$ determines the function uniquely and the algorithm is both convergent and stable. But in this case

$$
u_{n}(r)=u(r)-\frac{n^{-1}}{n^{-1 / 2}} \cdot v(r)\{1+o(I)\}, n \rightarrow \infty
$$


and it is apparent that the rate of convergence will be governed by the very slowly decreasing function $n^{-1 / 2}$. Suppose, however, that we give an additional initial value of $u(r)$. With two initial values our algorithm is equivalent to forward recurrence of the given difference equation and all questions of convergence disappear. This forward recurrence is, of course, unstable for the desired solution $u(r)$ but the instability is very weak; any smal1 component of the more dominant solution $v(r)$ can grow no faster than $r^{1 / 2}$ relative to $u(r)$. Although this rate of growth (or any other positive rate of growth) is ultimately sufficient to obliterate the desired solution when computed in finite precision arithmetic, subsequences of the solution of considerable length can be computed before this happens. Thus in many practical situations where the separation of solutions is weak, a minor modification ${ }^{\dagger}$ of the algorithm performs acceptably well with regard to both stability and convergence.

Finally, suppose that the relative separation of $u(r)$ and $v(r)$ is like $r^{-3 / 2}$ or $r^{-5 / 2}$ (compare Example 4.4 .1 which presents the computation of a function exhibiting both forms of behavior, $r^{-3 / 2}$ on the subsequence of odd terms and $\dot{r}^{-5 / 2}$ on the subsequence of even terms). These separation ratios are too weak to allow rapid convergence of the unmodified algorithm, and at the same time too strong for the numerical instability in the modified algorithm to be truly insignificant. Computation of cases such as this are the most difficult for the algorithm to perform well, and performance can be improved only by recourse to extended-precision computation.

\footnotetext{
†n general the modified algorithm will require a back substitution as well as a forward elimination stage. Nevertheless, similar considerations govern the trade-off between numerical instability and slow convergence.
} 
Perhaps it should be stressed that a complete knowledge of the asymptotic behavior of all of the solutions of the homogeneous equation is essential to determine the correct number of initial conditions. This information may be developed for a broad class of linear difference equations using known analytical results $(\$ 2.2)$. An alternative would be to attempt to construct the essential classification parameters - the number of distinct types of solution, and the number of linearly independent solutions of each type - by numerical experimentation. Under this approach, the algorithm presented in this thesis would be applied with chosen initial conditions appropriate to different possible realizations of the classification theory, that is, different possible forms of the optimally ranked basis. Presumably, incorrect choices would be revealed by discernible numerical instability. The effectiveness of such an approach has not be assessed, however.

In conclusion, the results presented in this thesis are distinguished from the work of earlier authors in the following ways. First, the interplay between linear difference operators and infinite band matrices achieves a fuller blending of analytic and algebraic ideas. This leads to a clearer understanding of stability, including the tradeoff choices that exist between slow convergence and mild instability when the asymptotic separation of adjacent solutions is weak. Secondly, the classification theory for separable operators is believed to be new. It enables the proper number of initial conditions that are prescribed by the convergence theorem to be determined directly from the asymptotic behavior of fundamental solutions of the given difference equation. In contrast, Zahar employs Eundamental solutions of the adjoint equation and Oliver applies a 
point-by-point growth criterion between pairs of solutions. Finally, the numerical examples presented here have been chosen in such a way that the solutions exhibit a much richer variety of growth rates than in any previous investigation. 
APPENDIX

Proof of Lema 2.1 .1

The proof consists of (i) identifying the sequence defined by equation $(2.1 .4)$ as an almost periodic sequence, and (ii) proving that every nonzero ainost pericdic sequence has an infinite subsequence which is bounded away from zero. We begin by presenting the essential elements of the theory of almost periodic functions.

Eirst we give the characterization of almost periodic functions that is saken as the definition by Corduneanu [ 7 ]. A function of the form

$$
I(x)=\sum_{k=1}^{n} c_{k} e^{i \lambda_{k} x},-\infty<x<\infty,
$$

where the $c_{k}$ are complex numbers, the $\lambda_{k}$ are real numbers and $i=\sqrt{-1}$, is called a complex trigonometric polynomial. Afunction $f(x)$ taking complex values and having all real numbers as domain is an almost pericdic Eunction provided that to each $\equiv>0$ there corresponds some trigonometric polynomial $T_{E}(x)$ such that

$$
\left|\hat{f}(x)-T_{\varepsilon}(z)\right|<\varepsilon
$$

uniformly on $-\infty<x<\infty$. Clearly, every complex trigononetric polynomial is itself an almost periodic function.

Aa alternative characterization is the definition of $\mathrm{H}$. Bohr [4]: A continuous complex valued function $f(x)$ defined on the real line is almost periodic if to each $\varepsilon>0$ there corresponds a positive real number $z=3(\Leftrightarrow)$ such that every open interval of the real line of length 2 contains at least one point E for which

$$
|f(x+\xi)-f(x)|<\varepsilon
$$


uniformly for every $x$. Any number $\xi$ which satisfies this condition is called a translation number of $f$ corresponding to $\varepsilon$, or more briefly, an $\underline{\text { E-translation number }}$ of $\underline{\underline{1}}$.

An $E$-translation number of $\bar{z}$ is "almost" a period of $f$. Every periodic function is almost periodic as well. If $p$ is the fundamental period of a periodic function $\equiv$, then $r p$ for $r=0, \pm 1, \pm 2, \ldots$ are translation numbers of $f$ for each $\varepsilon$. Bohr's definition generalizes the fact that the periods of a periodic function form an arithmetic progression.

Bohr's definition and Corduneanu's definition define exactly the same class of functions. This is the "fundamental theorem" of the theory of almost periodic functions. See either [4] or [7] for a full account. Turning now to seçuences, we have the following definition presented in $[7,31.6]$ : A function $f(r)$. caking complex values and having the set of integers as domain, is an almost period sequence provided that to each $\varepsilon>0$ there corresponds some positive integer $N=N(\varepsilon)$ such that, within any set of $\mathrm{N}$ consecutive integers, there exists an integer $P$ for which

$$
|\hat{I}(r+p)-\xi(r)|<\varepsilon .
$$

uniforaly over all integers $r$. Corduneanu proves "A necessary and sufficient condition for a sequence $\left\{a_{r}\right\}$ to be almost periodic is the existence of an almost periodic function $f(x),-\infty<x<\infty$, such that $a_{r}=f(r), \quad r=0, \pm 1 . \pm 2, \ldots "$; see $[7$, Theorem 1.27].

We are now ready to prove Lema 2.1.1. If $r$ were allowed to assume all real values, instead of being restricted to $r=0,1,2, \ldots$, then equation (2.1.4) deínes a trigcnometric polynomial. By Corduneanu's definition, every trigonometric polynomial is an almost periodic function. 
By the theorem quoted above, the sequence $x(r), r=0, \pm 1, \pm 2, \ldots$ deined by equation (2.1.4) is an almost periodic sequence. The only thing remaining is to prove the following assertion:

If an almost periodic sequence $\{f(r)\}$ is not identically zero, then there exists an infinite subsequence of $\{f(r)\}$ which is bounded away from zero.

Proof Assume $\{f(r)\}$ is not identically zero. Let $r_{0}$ be such that $f\left(r_{0}\right) \neq 0$ and put $A=\left|f\left(r_{0}\right)\right|$. Put $\varepsilon=A /(2+A)$. Then $\varepsilon \in(0,1)$, and by the definition of an almost periodic sequence there exists a positive integer $N_{1}$, say, such that for some integer $P_{1} \in\left\{1,2, \ldots, N_{1}\right\}$ the inequality

$$
\left|f\left(r_{0}+p_{1}\right)-f\left(r_{0}\right)\right|<\varepsilon
$$

is satisfied. Put $r_{1}=r_{0}+p_{I}$. Next, there exists a positive integer $\mathrm{N}_{2}$ such that for some integer $\mathrm{P}_{2} \leqslant\left\{1,2, \ldots, \mathrm{N}_{2}\right\}$ the inequality

$$
\left|f\left(r_{1}+p_{2}\right)-f\left(r_{1}\right)\right|<\varepsilon^{2}
$$

is satisfied. Put $r_{2}=r_{1}+p_{2}$. Continuing in this manner, we see that for each $k=3,4,5, \ldots$ we can find an integer $r_{k}>r_{k-1}$ such that

$$
\left|f\left(r_{k}\right)-f\left(r_{k-1}\right)\right|<\varepsilon^{k} .
$$

For each $k=1,2,3, \ldots$ we have

$$
\begin{aligned}
\left|f\left(r_{k}\right)-f\left(r_{0}\right)\right| & \leq \sum_{j=1}^{k}\left|f\left(r_{j}\right)-f\left(r_{j-1}\right)\right| \\
& <\sum_{j=1}^{k} \varepsilon^{j}<\frac{\varepsilon}{1-\varepsilon}<\frac{1}{2} A .
\end{aligned}
$$


157

Since $A=\left|f\left(r_{0}\right)\right|$, we have

$$
\begin{aligned}
\left|f\left(r_{k}\right)\right| & =\left|f\left(r_{0}\right)+f\left(r_{k}\right)-f\left(r_{0}\right)\right| \\
& \geq A-\left|f\left(r_{k}\right)-f\left(r_{0}\right)\right|>\frac{1}{2} A,
\end{aligned}
$$

which finishes the proof of the assertion. 


\section{REFERENCES}

I. Aggarwal, V. B. and Burgmeier, J.W. An algorithmic decomposition system for second order recurrence relations, BIT 18, 241-258 (1978).

2. Birknoff, G. D. Formal theory of irregular linear difference equations, Acta Math. 54, 205-246 (1930).

3. Birkhoff, G. D., and Trjitzinsky, W. J. Analytic theory of singular difference equations, Acta Math. 60, 1-89 (1933).

4. Bohr, H. Almost Periodic Functions. Chelsea, Bronx, New York, 1951. (Translated from the German edition of 1933.)

5. British Association for the Advancement of Science, Mathematical Tables, v. 10, Bessel Functions. Part II. Functions of Positive Integer Order. Cambridge University Press, London and New York, 1952.

6. Cash, J.R. An extension of Olver's method for the numerical solution of linear recurrence relations, Math. Comp. 32, 497-510 (1978).

7. Corduneanu, C. Almost Periodic Functions. Interscience, New York, 1968. (Translated from the Romanian edition of 1961.)

8. Dongarra, J. J., Bunch, J.R., Moler, C. B., and Stewart, G.W. LINPACK Users' Guide. SIAM Publications, Philadelphia, Pa., 1979.

9. Gautschi, W. Computational aspects of three-term recurrence relations, SIAM Review 9, 24-82 (1967).

10. Isaacson, E. and Keller, H. B. Analysis of Numerical Methods. Wiley, New York, 1966.

11. Lozier, D. W., and Smith, J. M. Algorithm XXX. Extended-range arithmetic and normalized Legendre polynomials, to appear in ACM Trans. on Math. Software.

12. Massachusetts Institute of Technology, MACSYMA Reference Manual. Mathlab Group, Laboratory for Computer Science, Cambridge, MA 02139, 1977. 
13. Miller, K. S. Linear Difference Equations. Benjamin, New York, 1968.

14. Milne-Thomson, L. M. The Calculus of Finite Differences, Macmillan, Lond on, 1933.

15. Muir, T. A Treatise on the Theory of Determinants. Dover, New York, 1960 .

16. National Bureau of Standards, Handbook of Mathematical Functions, Applied Math. Series No. 55, (M. Abramowitz and I. A. Stegun, Eds.). U. S. Govt. Printing Office, Washington, D. C., 1964.

17. National Bureau of Standards, Tables of Associated Legerdre Functions. Columbia University Press, New York, 1945.

18. Oliver, J. Relative error propagation in the recursive solution of linear recurrence relations, Numer. Math. 9, 323-340 (1967).

19. Oliver, J. The numerical solution of linear recurrence relations, Numer. Math. 11, 349-360 (1968).

20. Oliver, J. An extension of Olver's error estimation technique for linear recurrence relations, Numer. Math. 12, 459-467 (1968).

21. Olver, F. W. J. Numerical solution of second-order linear difference equations, J. Res. National Bureau of Standards 71B. 111-129 (1967).

22. OIver, F. W. J. and Sookne, D. J. Note on backward recurrence algorithms, Math. Comp. 26, $941-947$ (1972).

23. Robinson, C. A numerical and analytical investigation of Struve's function. Thesis, London University, 1948.

24. Smith, I. M., Olver, F. W. J., and Lozier, D. W. Extended-range arithmetic and normalized Legendre polynomials, to appear in ACM Trans. on Math. Software. 
25. Van der Cruyssen, P. A reformulation of Olver's algorithm for the numerical solution of second-order linear difference equations, Numer. Math. 32, 159-166 (1979).

26. Wimp, J. On Recursive Computation, ARL 69-0186, Aerospace Research Laboratories, Office of Aerospace Research, U. S. Air Force, WrightPatterson AFB, Ohio, November 1969.

27. Zahar, R. V. M. Computational Algorithms for Linear Difference Equations. Thesis, Purdue University, January 1968.

28. Zahar, R. V. M. A mathematical analysis of Miller's algorithm, Numer. Math. 27, 427-447 (1977). 
NBS.II AA IREV. 9.78)

\begin{tabular}{|c|c|c|c|}
\hline $\begin{array}{l}\text { U.S. DEPT. OF COMM. } \\
\text { BIBLIOGRAPHIC DATA } \\
\text { SHEET }\end{array}$ & $\begin{array}{l}\text { 1. PUBLICATION OR REPORT NO. } \\
\text { NBSIR } 80-1976\end{array}$ & 2.Gor't Accession No. & \%. \\
\hline \multirow{2}{*}{\multicolumn{3}{|c|}{ Numerical Solution of Linear Difference Equations }} & $\begin{array}{l}\text { 5. Publication Date } \\
\text { MARCH, } 1980\end{array}$ \\
\hline & & & 6. Performing Organization Code \\
\hline \multicolumn{3}{|c|}{ Daniel W. Lozier } & 8. Performing Organ. Report No. \\
\hline \multirow{2}{*}{\multicolumn{3}{|c|}{$\begin{array}{l}\text { NATIONAL BUREAU OF STANDARDS } \\
\text { DEPARTMENT OF COMMERCE } \\
\text { WASHINGTON, DC } 20234\end{array}$}} & 10. Project/TaskAork Unit No \\
\hline & & & 11. Contract/Grant No. \\
\hline \multirow{2}{*}{\multicolumn{3}{|c|}{ 12. SPONSORING ORGANIZATION NAME AND COMPLETE ADDRESS (street, city, state, zIP) }} & $\begin{array}{l}\text { 13. Type of Report \& Period Covered } \\
\text { Final }\end{array}$ \\
\hline & & & 14. Sponsoring Agency Code \\
\hline
\end{tabular}

15. SUPPLEMENTARY NOTES

Document describes a computer program; SF-185, FIPS Software Summary, is attached.

16. ABSTRACT (A 200-word or less tactual summary of most significant information. If document includes a significant bibliography or

Consider a given linear difference equation $\sum_{s=0}^{\ell} d_{s}(r) y(r+s)=g(r)$ where $\ell \geq 2$ and $r=0,1,2, \ldots$. Suppose $y$ is a solution of this equation and $u, v$ are solutions of the homogeneous form of this equation such that $u(r) / v(r) \rightarrow 0, y(r) / v(r) \rightarrow 0, u(r) / y(r) \rightarrow 0$. Under these circumstances algorithms for the computation of $y$ based on forward or backward recurrence, such as the Miller algorithm, are numerically unstable.

Stable algorithms, such as the method of Olver, have been based on approximating $y(r)$ by the solutions of a certain sequence of boundary value problems. Specifically, $y_{n}(r)$ is a solution that coincides with $y(r)$ over some initial range of $r$, say $r=i$, $i+1$, ..., $i+j-1$, and satisfies $y_{n}(r)=0$ for $r=n, n+1, \ldots, n+l-j-1$. Here $j$ is an integer whose value depends on the asymptotic behavior of the chosen solution $y(r)$ and $n$ is an arbitrary large integer. Boundary value problems of this type are shown to be equivalent to two initial value problems of order $j$ and $l-j$ by factorization of the difference operator. The solution of the problem of order $j$ is obtained by forward recurrence; the solution of the other problem is obtained by backward recurrence.

The algorithm is specified completely for a broad class of operators including, for example, every constant-coefficient operator. Convergence of $y_{y}(r)$ to y $(r)$ as $n \rightarrow \infty$ for Fixed $r$ is proved and an expansion of the truncation error is derived. Numerical stability is demonstrated. The method is tested by numerical examples involving fourthorder equations with variable coefficients.

17. KEY WORDS (six to twelve entries; alphabotical order; capitalize only the first letter of the first key word unless a propor namo;

separated by semicolons)

Factorization methods; linear difference equations; 1inear recurrence relations; Miller's algorithm; Olver's algorithm; numerical stability.

18. AVAILABILITY Enlimited

For Official Distribution. Do llot Release to NTIS

Order From Sup. of Doc., U.S. Government Printing Office, Washington, DC 20402, SD Stock No. SNOO3-003-

[ Order From National Technical information Service (NTIS), Springfield, VA. 22161
19. SECURITY CLASS (THIS REPORT)

UNCLASSIFIED

20. SECURITY CLASS

(THIS PAGE)

UNCLASSIFIED
21. NO. OF PRINTED PAGES

156

22. Price

$\$ 11.00$ 


